\title{
Design and Implementation of a Gait Rehabilitation Robot Manipulator
}

By

\section{F. Adam Mullins}

\author{
A thesis submitted to \\ The Faculty of Graduate Studies and Research \\ in partial fulfilment of \\ the degree requirements of \\ Masters of Applied Science - Mechanical Engineering
}

Ottawa-Carleton Institute for

Mechanical and Aerospace Engineering

Department of Mechanical and Aerospace Engineering

Carleton University

Ottawa, Ontario, Canada

September 2010

Copyright $\mathbb{C}$

2010 - F. Adam Mullins 


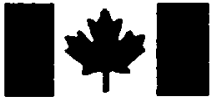

Library and Archives

Canada

Published Heritage

Branch

395 Wellington Street

Ottawa ON K1A ON4

Canada
Bibliothèque et

Archives Canada

Direction du

Patrimoine de l'édition

395 , rue Wellington

Ottawa ON K1A ON4

Canada
Your file Votre référence

ISBN: 978-0-494-79543-9

Our file Notre référence

ISBN: 978-0-494-79543-9
NOTICE:

The author has granted a nonexclusive license allowing Library and Archives Canada to reproduce, publish, archive, preserve, conserve, communicate to the public by telecommunication or on the Internet, loan, distribute and sell theses worldwide, for commercial or noncommercial purposes, in microform, paper, electronic and/or any other formats.

The author retains copyright ownership and moral rights in this thesis. Neither the thesis nor substantial extracts from it may be printed or otherwise reproduced without the author's permission.
AVIS:

L'auteur a accordé une licence non exclusive permettant à la Bibliothèque et Archives Canada de reproduire, publier, archiver, sauvegarder, conserver, transmettre au public par télécommunication ou par l'Internet, prêter, distribuer et vendre des thèses partout dans le monde, à des fins commerciales ou autres, sur support microforme, papier, électronique et/ou autres formats.

L'auteur conserve la propriété du droit d'auteur et des droits moraux qui protège cette thèse. $\mathrm{Ni}$ la thèse ni des extraits substantiels de celle-ci ne doivent être imprimés ou autrement reproduits sans son autorisation.
In compliance with the Canadian Privacy Act some supporting forms may have been removed from this thesis.

While these forms may be included in the document page count, their removal does not represent any loss of content from the thesis.
Conformément à la loi canadienne sur la protection de la vie privée, quelques formulaires secondaires ont été enlevés de cette thèse.

Bien que ces formulaires aient inclus dans la pagination, il n'y aura aucun contenu manquant. 


\section{Abstract}

Every year strokes, spinal cord injuries and neurodegenerative diseases cause people to begin gait rehabilitation in order to relearn how to walk after these debilitating injuries. Gait rehabilitation requires intensive physiotherapy sessions and is extremely taxing on both the patient and the physiotherapists. Robotics represents an opportunity to relieve the physical toll on the therapist while also providing a more exact motion for the patient.

From limitations in the current assortment of gait rehabilitation robots a novel platform was developed by the Advanced Biomechatronics Laboratory (ABL) at Carleton University called the Virtual Gait Rehabilitation Robot (ViGRR). Currently developed as a prototype platform for testing a novel rehabilitation configuration and rehabilitation control schemes, this thesis presents the design and implementation of the ViGRR manipulator.

This thesis describes the design of a four degrees of freedom planar manipulator for the purposes of haptic gait rehabilitation. From the kinematic and dynamic model of human gait the end effector trajectory and loading requirements were determined and applied to the structural design and optimization. The iterative optimization determined the actuation components and robot parameters. The manipulator was designed and analyzed using a finite element analysis and manufactured. The assembled manipulator was then kinematically and dynamically calibrated and tested in position control and force control modes to verify the calibrations and controllers.

The realized platform provides an opportunity for the development of novel controls in haptic gait rehabilitation as well as future objectives to develop the manipulator platform into a fully realized, commercial gait rehabilitation trainer. 


\section{Acknowledgements}

I would first like to thank my supervisor Mojtaba Ahmadi for the opportunity to work on such an innovative project that will hopefully one day assist so many people in such a positive manner. This project would not have been possible without my colleague, Kyle Chisholm, who worked long hours to develop the control architecture of our project. I have learned much during my studies with the both of you and this project was a once-ina-lifetime opportunity. I would be remiss not to acknowledge Kevin Sangster and Alex Proctor for the good cheer during all of the long hours I spent in the machine shop. I would like to thank my family for their constant support and encouragement throughout the entire process. Finally, for being there every step of the way to pick me up and push me forward, even from the other side of the world, thank you Erin. 


\section{Table of Contents}

$\begin{array}{ll}\text { Abstract } & \text { iii }\end{array}$

Acknowledgements iv

Table of Contents $\quad v$

List of Tables $\quad$ ix

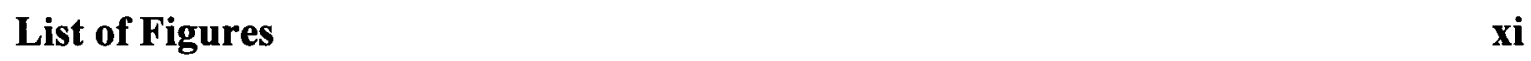

$\begin{array}{ll}\text { Chapter 1: Introduction } & 1\end{array}$

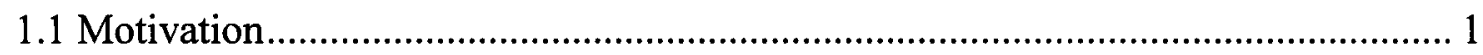

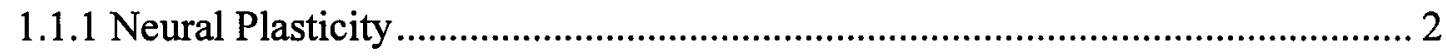

1.1.2 Motor Learning as it Applies to Rehabilitation ........................................... 2

1.2 Virtual Gait Rehabilitation Robot ................................................................ 6

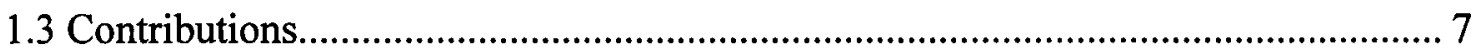

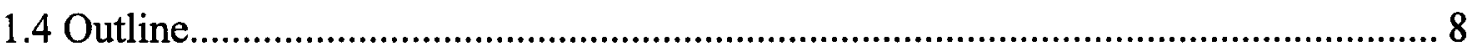

Chapter 2: Evaluation of Current Technologies in Gait Rehabilitation 10

2.1 Body Weight Supported Treadmill Training ............................................... 10

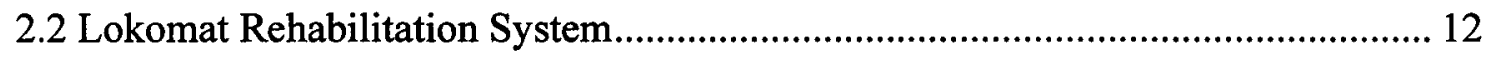

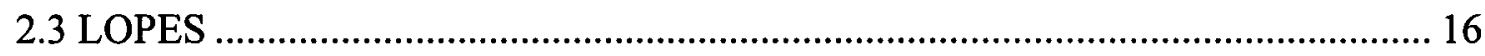

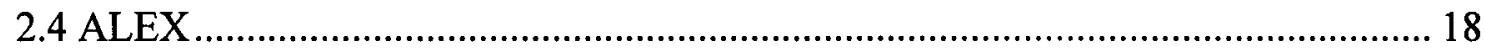

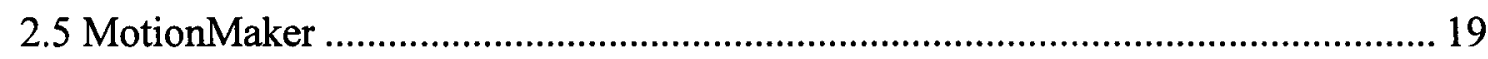

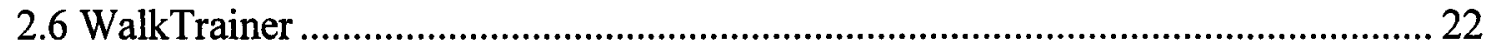

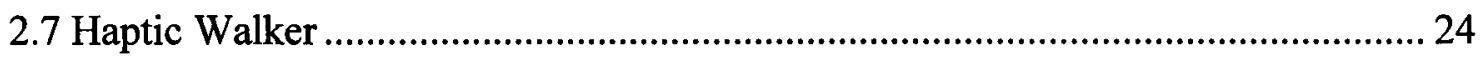

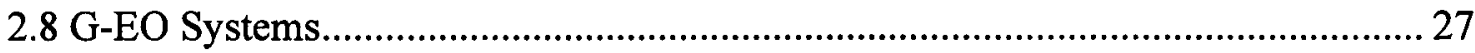




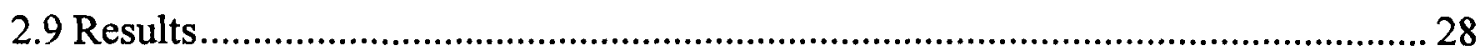

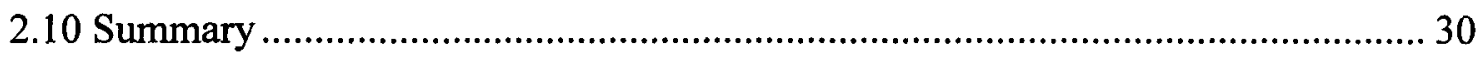

$\begin{array}{ll}\text { Chapter 3: Platform Conceptualization and Design } & 31\end{array}$

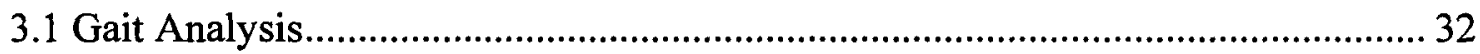

3.2 Concept Platform Design Details......................................................................... 44

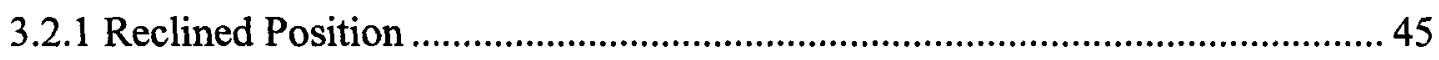

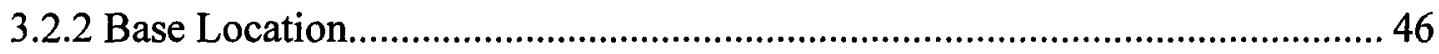

3.2.3 Single Point Attachment at End Effector....................................................... 47

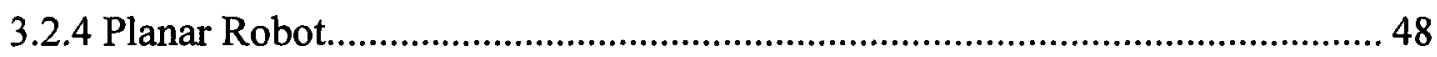

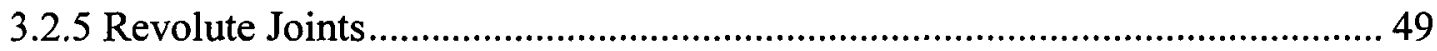

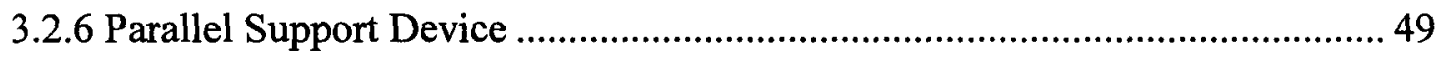

3.2.7 Four Degrees of Freedom ................................................................................ 49

3.3 Development of Robot Model ....................................................................... 50

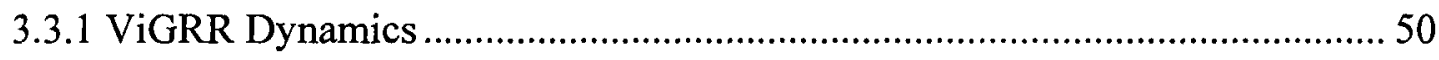

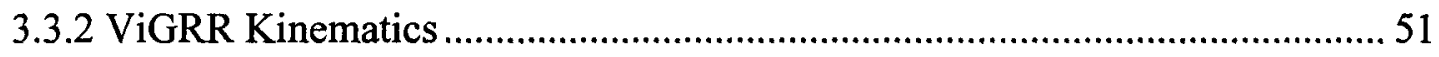

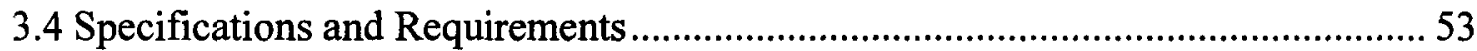

3.4.1 Patient Workspace Requirement....................................................................... 54

3.4.2 Trajectory and Loading Requirements............................................................. 55

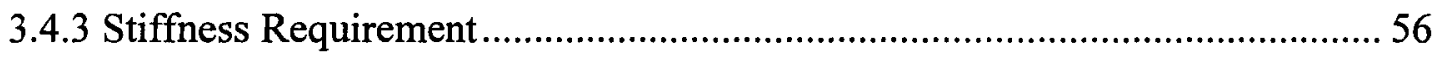

3.4.4 Safety Requirements ..................................................................................... 56

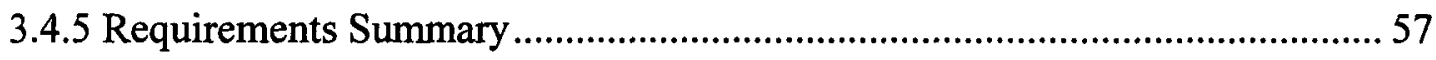

3.5 Optimal Design and Component Selection Process.............................................. 57

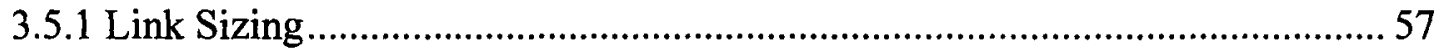

3.5.2 Motor and Gear Selection .......................................................................... 59

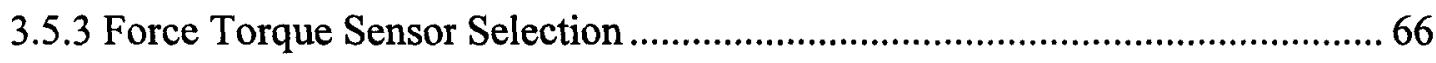

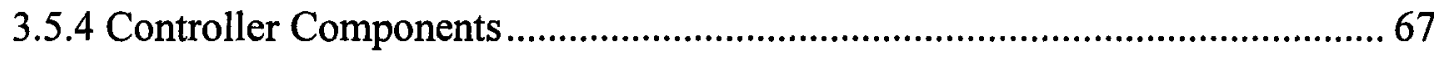

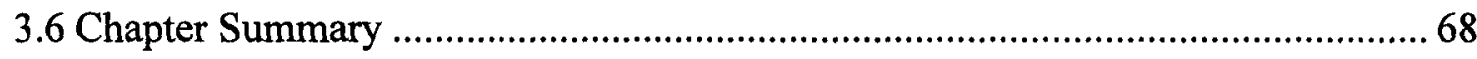


Chapter 4: Design and Structural Analysis of Virtual Gait Rehabilitation Robot Manipulator

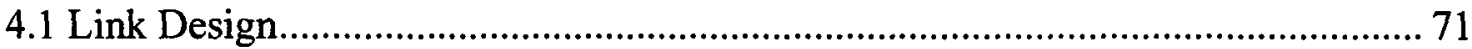

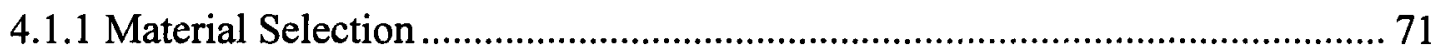

4.1.2 Link Cross Section Selection ..................................................................... 73

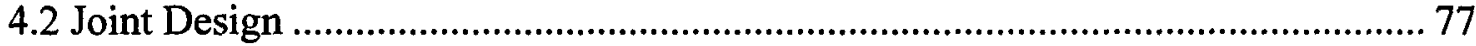

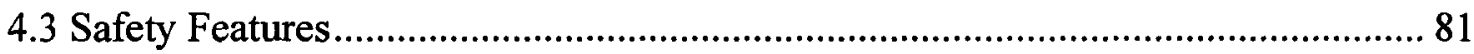

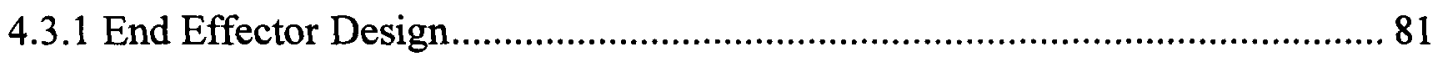

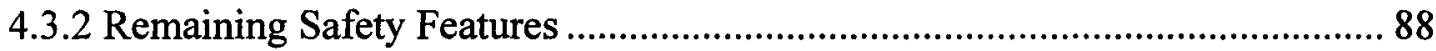

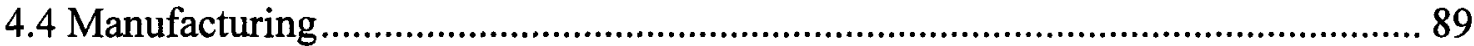

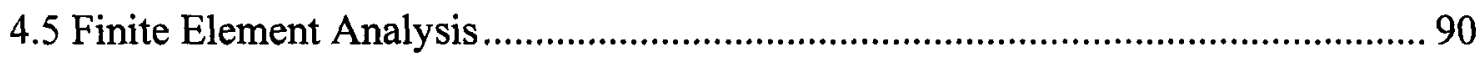

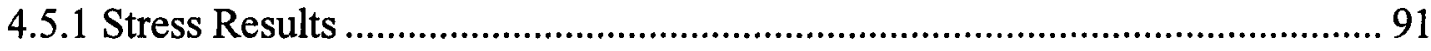

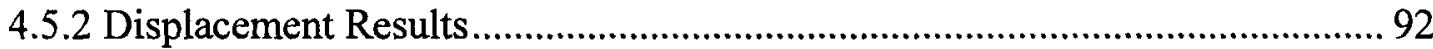

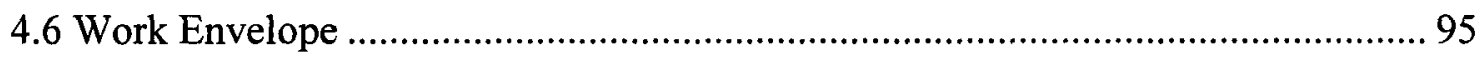

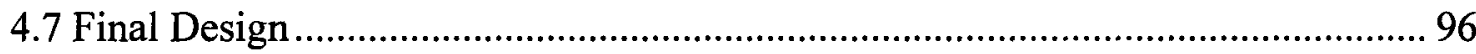

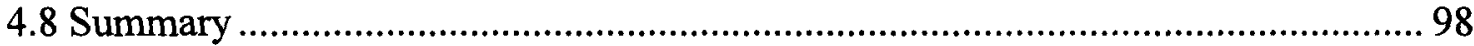

Chapter 5: Calibration and Experimentation 99

5.1 Overview of ViGRR Controller................................................................. 99

5.1.1 Trajectory Generation and Position Control ........................................... 100

5.1.2 Admittance Controller ............................................................................. 101

5.2 Single Degree of Freedom Parameter Identification ..................................... 101

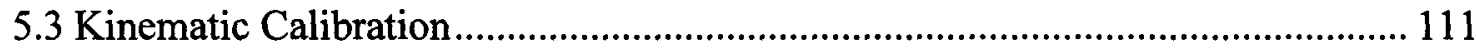

5.4 Four Degrees-of-Freedom Dynamic Parameter Identification .......................... 114

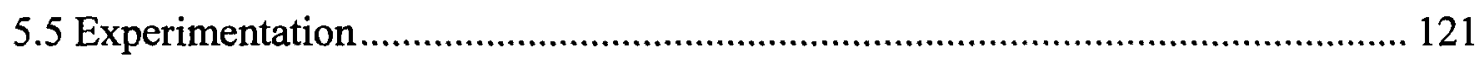

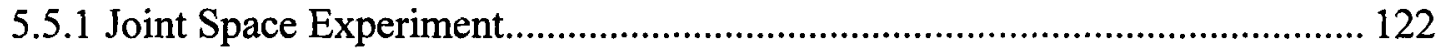

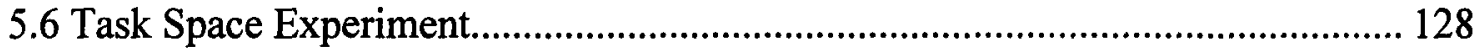

5.7 Admittance Control Experiment ............................................................. 133

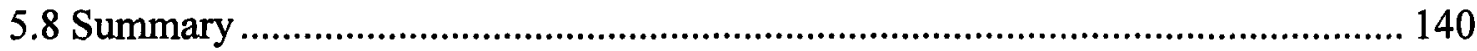




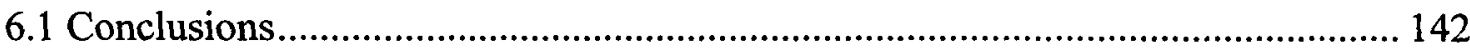

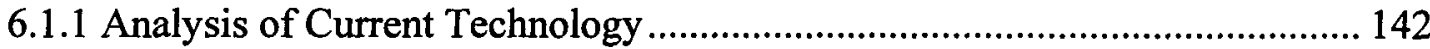

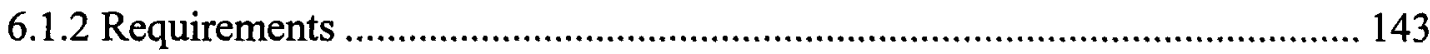

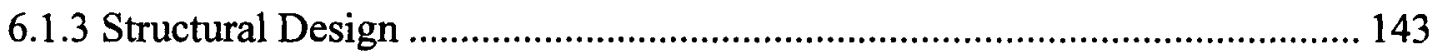

6.1.4 Manipulator and Structural Analysis ..................................................... 143

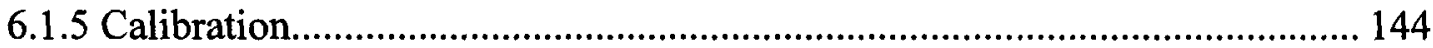

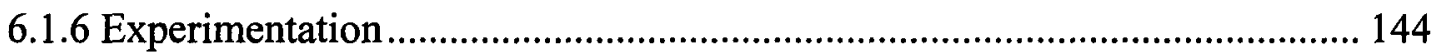

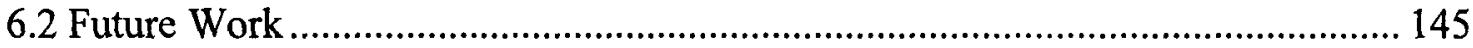

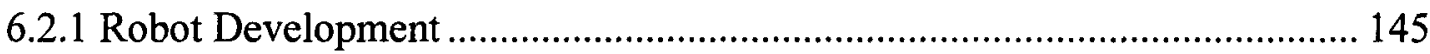

6.2.2 Controller Development and Clinical Trials........................................... 146

Chapter 7: Bibliography 147 


\section{List of Tables}

Table 1.1: Ten Principles of Experience-Dependent Plasticity ........................................... 3

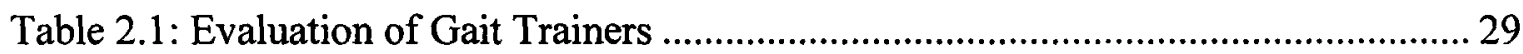

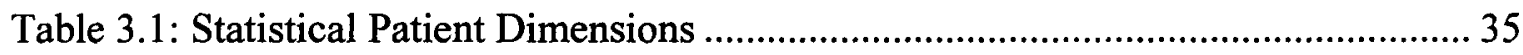

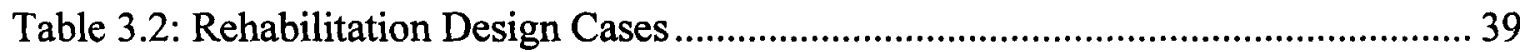

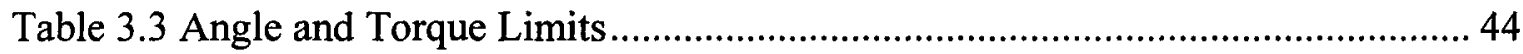

Table 3.4: Optimized Design Values ................................................................................ 59

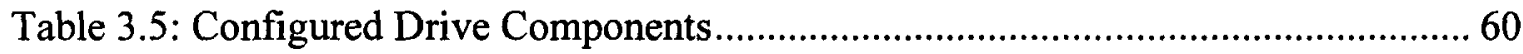

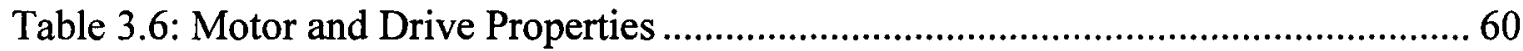

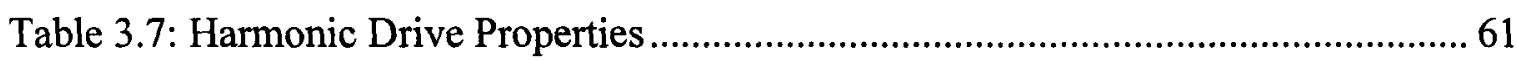

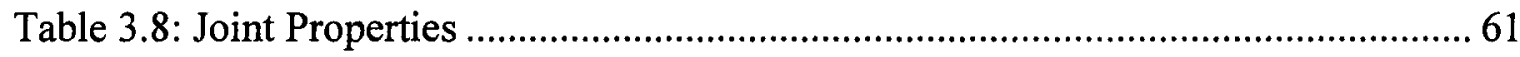

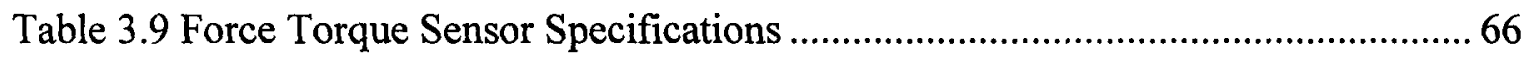

Table 4.1 Material Considered in Design Process.......................................................... 72

Table 4.2: Results of Cross Section Analysis ................................................................. 74

Table 4.3: Estimated Mass Properties of Links from CAD Model.................................... 75

Table 4.4: FEA Results of Various Joint Designs ............................................................ 79

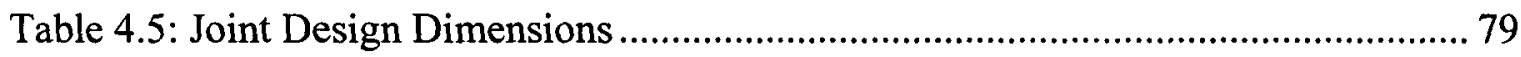

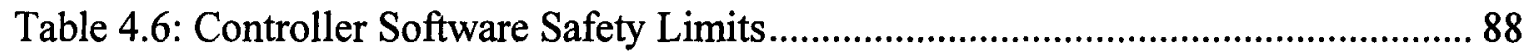

Table 4.7: Loading Condition for Finite Element Analysis................................................ 91

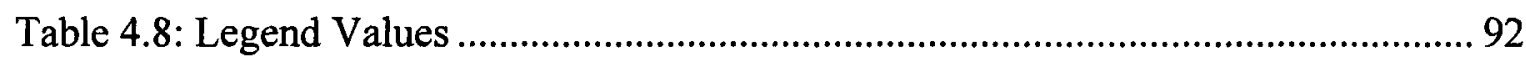

Table 4.9: Displacement Results.................................................................................. 95

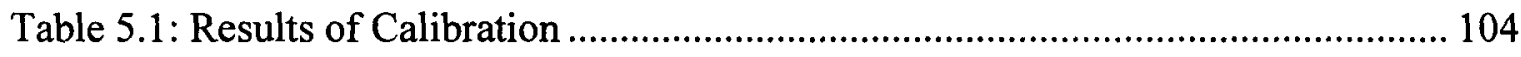

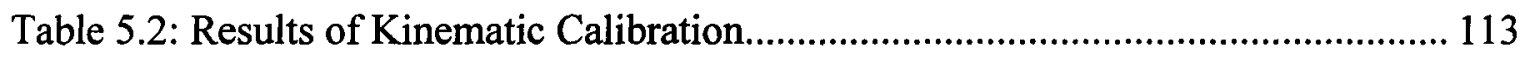

Table 5.3: Torque Capacity of Joints ............................................................................. 121

Table 5.4: Joint Space Motion Values ............................................................................. 123 


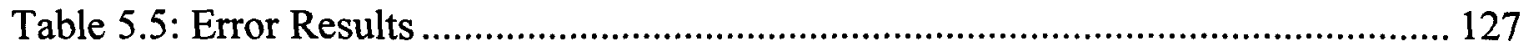

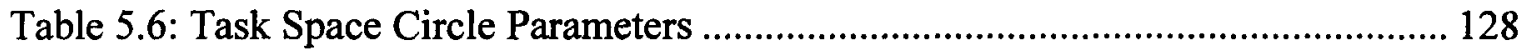

Table 5.7: End Effector Tracking Error Results ...................................................... 133

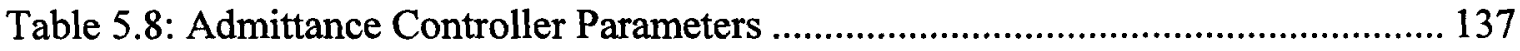




\section{List of Figures}

Figure 1.1: Brain Surface Allocation of Normal and Stimulated Finger Use in Adult Owl

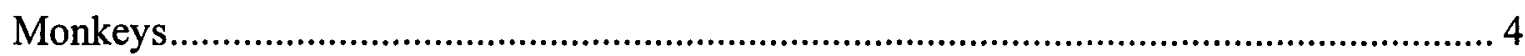

Figure 2.1: Body Weight Supported Treadmill Training.................................................... 11

Figure 2.2: Layout of the Lokomat System ...................................................................... 14

Figure 2.3: Back View of the Lokomat Gait Trainer......................................................... 14

Figure 2.4: Close up of Lokomat Actuators....................................................................... 15

Figure 2.5: LOPES Degrees of Freedom .................................................................. 16

Figure 2.6: Layout of the Degrees of Freedom of the LOPES system ............................. 17

Figure 2.7: Design of the LOPES Knee joint articulator .................................................. 18

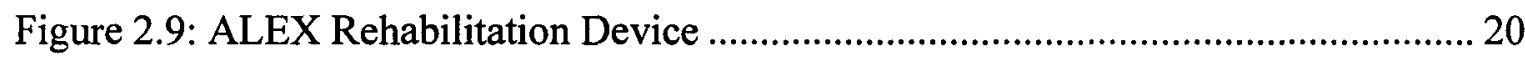

Figure 2.8: ALEX System in Use with Visual Feedback ............................................. 20

Figure 2.10: MotionMaker Platform with FES Driven Actuation ...................................... 21

Figure 2.11: Design of MotionMaker Knee Joint with Servo Motor Powered Ball Screw

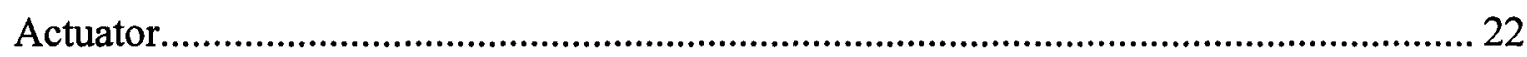

Figure 2.12: WalkTrainer in use by Healthy participant ............................................. 23

Figure 2.13: Layout of WalkTrainer Mobile Platform ................................................. 24

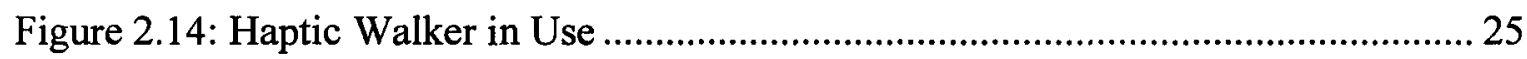

Figure 2.15: Haptic Walker Footplate Design ................................................................... 26

Figure 2.16: G-EO Gait Motion Device ………………….......................................... 27

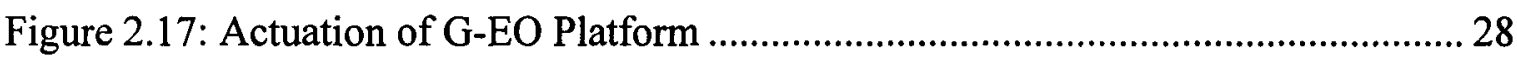

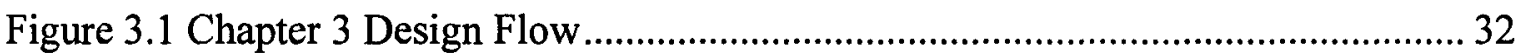

Figure 3.2: Vertical Ground Reaction Force vs. Percent of Gait Cycle ........................... 33

Figure 3.3: Horizontal Ground Reaction Force vs. Percent of Gait Cycle .......................... 34

Figure 3.4 Hip Joint Angle vs Gait Cycle .................................................................... 36 


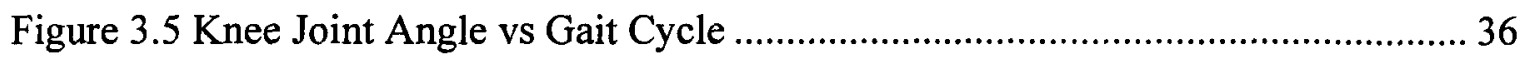

Figure 3.6 Ankle Joint Angle vs Gait Cycle ................................................................... 37

Figure 3.7: Hip Joint Moment vs. Percent of Gait Cycle.................................................... 37

Figure 3.8: Knee Joint Moment vs. Percent of Gait Cycle …………………………….. 38

Figure 3.9: Ankle Joint Moment vs. Percent of Gait Cycle............................................ 38

Figure 3.10 Body Positions for Rehabilitation ............................................................... 39

Figure 3.11 End Effector Spatial Position vs Time ......................................................... 40

Figure 3.12 End Effector Angular Position vs Time ...................................................... 40

Figure 3.13 End Effector Spatial Velocity vs Time........................................................... 41

Figure 3.14 End Effector Angular Velocity vs Time...................................................... 41

Figure 3.15 End Effector Spatial Acceleration vs Time ................................................ 42

Figure 3.16: End Effector Angular Acceleration vs Time ............................................... 42

Figure 3.17 End Effector Forces vs Time..................................................................... 43

Figure 3.18 End Effector Torques vs Time ................................................................. 44

Figure 3.19 Reclined User Rehabilitation Position.......................................................... 45

Figure 3.20: Aligned Manipulator .................................................................................... 46

Figure 3.21: Ground Mounted Manipulator..................................................................... 47

Figure 3.22: Side Mounted Manipulator......................................................................... 47

Figure 3.23: Anthropomorphic Design ...................................................................... 48

Figure 3.24: Frame, Length and Mass Definitions for the 4R ViGRR Redundant

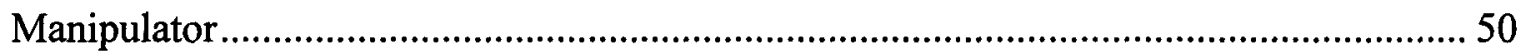

Figure 3.25: Overview of ViGRR Requirements ....................................................... 54

Figure 3.26: Work envelopes of a $95^{\text {th }}$ percentile man and a $5^{\text {th }}$ percentile female.......... 55

Figure 3.27: Component Selection Iterative Optimization .............................................. 58

Figure 3.28: End Effector Offset Angle........................................................................ 59

Figure 3.29: Motor Sizing - Joint 1 Torque ......................................................................... 62

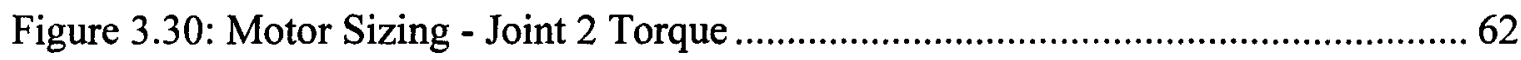

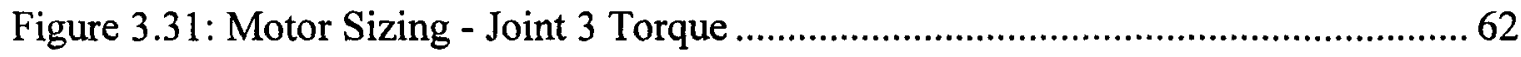

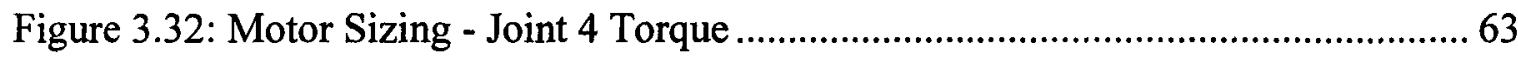




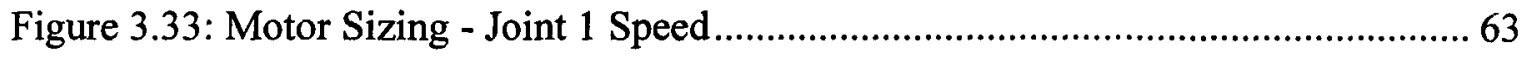

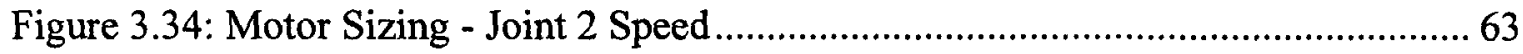

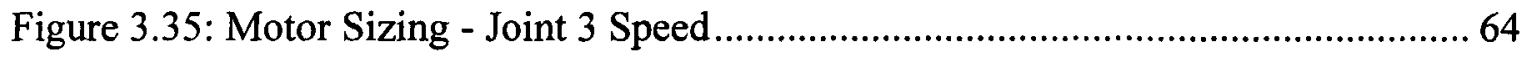

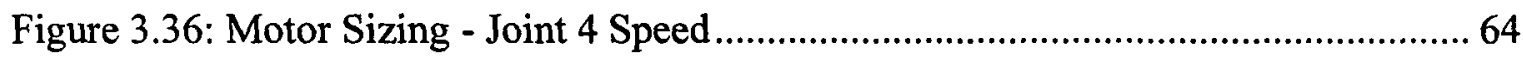

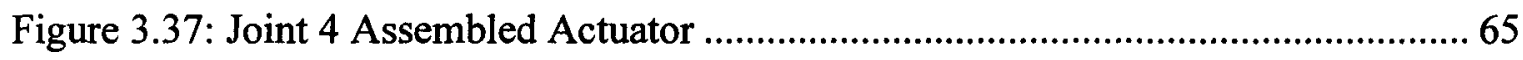

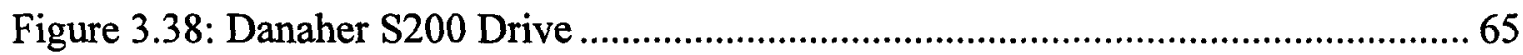

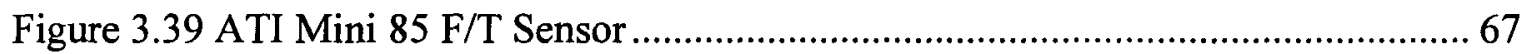

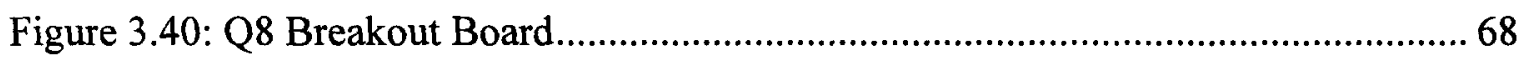

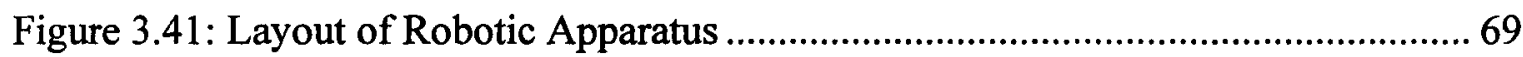

Figure 4.1: ViGGR Manipulator.................................................................................. 71

Figure 4.2: ViGRR Structural Design Process ............................................................. 72

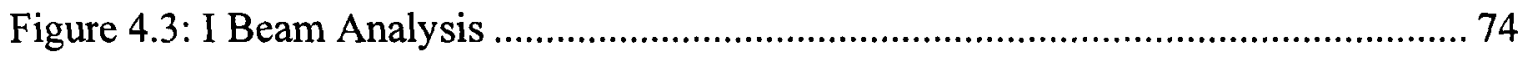

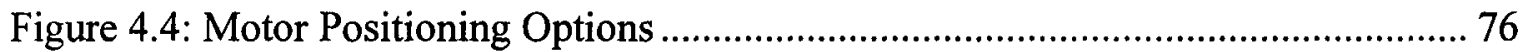

Figure 4.5: Link 2 with Actuators installed ................................................................... 76

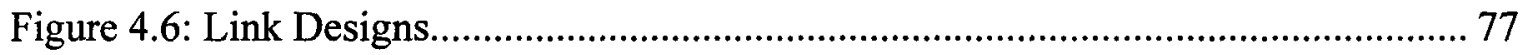

Figure 4.7: Final Joint 2 Stiffener Design............................................................... 78

Figure 4.8: Joint Design Evolution ......................................................................... 80

Figure 4.9: End Effector Angle and Coordinate System .............................................. 82

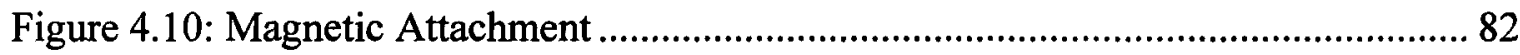

Figure 4.11: Filtered Force Torque Sensor Force Noise in X Direction .......................... 83

Figure 4.12: Filtered Force Torque Sensor Force Noise in Y Direction ........................... 83

Figure 4.13: Filtered Force Torque Sensor Force Noise in Z Direction........................... 84

Figure 4.14: Filtered Force Torque Sensor Torque Noise about X Axis........................... 84

Figure 4.15: Filtered Force Torque Sensor Torque Noise about Y Axis........................... 85

Figure 4.16: Filtered Force Torque Sensor Torque Noise about $Z$ Axis .......................... 85

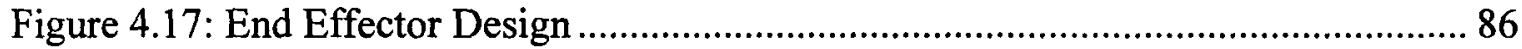

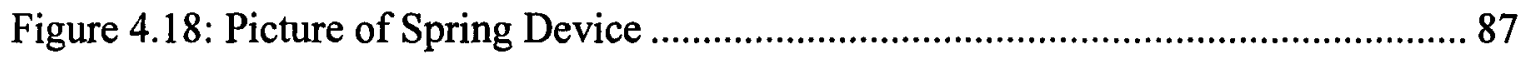

Figure 4.19: Tool Path Creation.................................................................................. 90

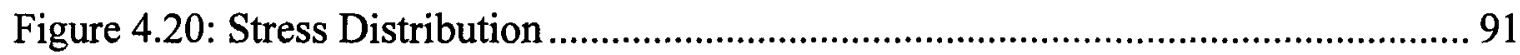


Figure 4.21: Location of Maximum Stress in Joint 2 ................................................. 92

Figure 4.22: Displacement Magnitude ........................................................................ 93

Figure 4.23: Displacement in X Direction..................................................................... 94

Figure 4.24: Displacement in Y Direction................................................................. 94

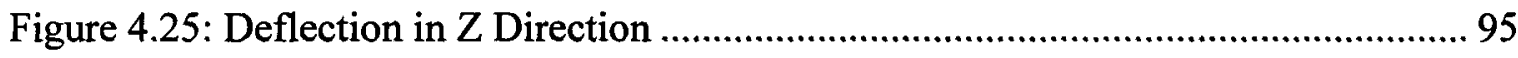

Figure 4.26: Workspace of Manipulator, Neglecting Collisions with Table ..................... 96

Figure 4.27: Final CAD Design of Structure …………................................................ 97

Figure 4.28: Images of Design of Manipulator................................................................. 98

Figure 5.1: Control Scheme for ViGRR ....................................................................... 100

Figure 5.2: Measured and Modeled Joint 1 Torques - Single DoF Calibration............... 104

Figure 5.3: Measured and Modeled Joint 2 Torques - Single DoF Calibration............... 105

Figure 5.4: Measured and Modeled Joint 3 Torques - Single DoF Calibration............... 106

Figure 5.5: Measured and Modeled Joint 4 Torques - Single DoF Calibration............... 106

Figure 5.6: Error in Joint 1 Torques - Single DoF Calibration ...................................... 107

Figure 5.7: Error in Joint 2 Torques - Single DoF Calibration ...................................... 107

Figure 5.8: Error in Joint 3 Torques - Single DoF Calibration ...................................... 108

Figure 5.9: Error in Joint 4 Torques - Single DoF Calibration ...................................... 108

Figure 5.10: Close up of Joint 1 Torque Data................................................................... 109

Figure 5.11: Close up of Joint 2 Torque Data................................................................. 109

Figure 5.12: Close up of Joint 3 Torque Data................................................................ 110

Figure 5.13: Close up of Joint 4 Torque Data.................................................................. 110

Figure 5.14: Kinematic Calibration Setup ................................................................... 112

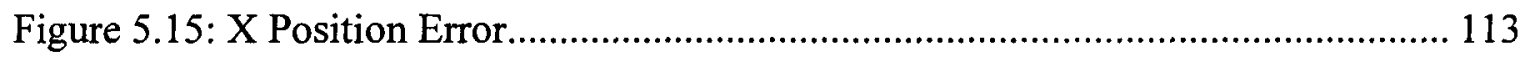

Figure 5.16: Y Position Error....................................................................................... 114

Figure 5.17: Angle Orientation Error........................................................................ 114

Figure 5.18: Measured and Modeled Joint 1 Torques ................................................... 117

Figure 5.19: Measured and Modeled Joint 2 Torques .................................................. 117

Figure 5.20: Measured and Modeled Joint 3 Torques ................................................... 118

Figure 5.21: Measured and Modeled Joint 4 Torques .................................................. 118 


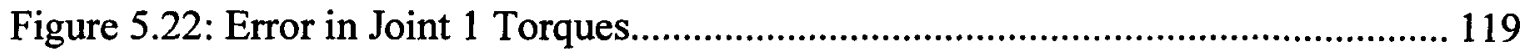

Figure 5.23: Error in Joint 2 Torques....................................................................... 119

Figure 5.24: Error in Joint 3 Torques............................................................... 120

Figure 5.25: Error in Joint 4 Torques................................................................ 120

Figure 5.26: Control Strategy for Joint and Task Space Experiment .......................... 122

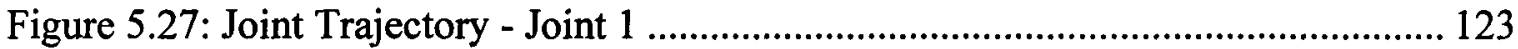

Figure 5.28: Joint Trajectory - Joint 2 ............................................................. 124

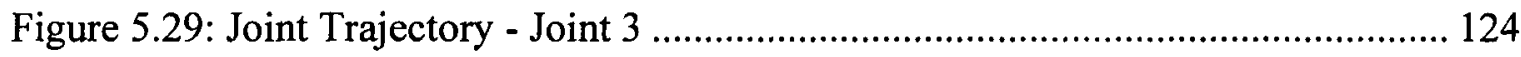

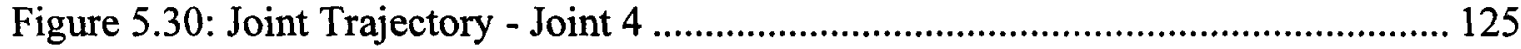

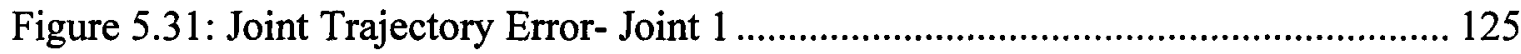

Figure 5.32: Joint Trajectory Error- Joint 2 ................................................... 126

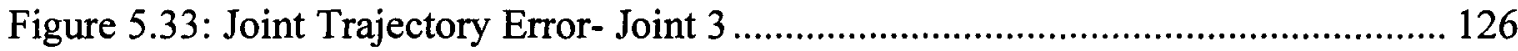

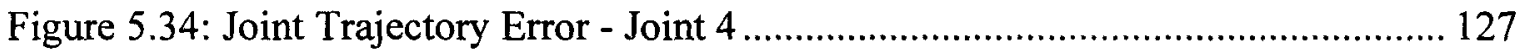

Figure 5.35: End Effector Position Tracking ...................................................... 129

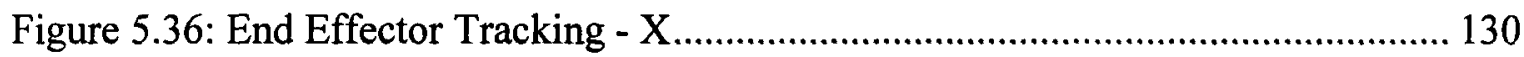

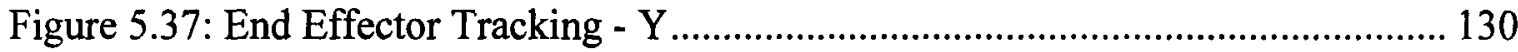

Figure 5.38: End Effector Tracking - Orientation ............................................... 131

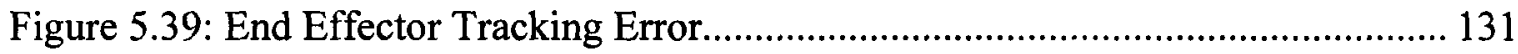

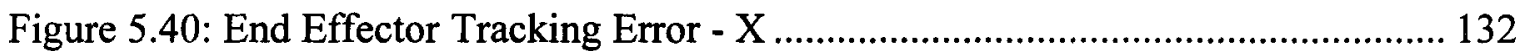

Figure 5.41: End Effector Tracking Error - Y.................................................... 132

Figure 5.42: End Effector Tracking Error - Orientation ........................................ 133

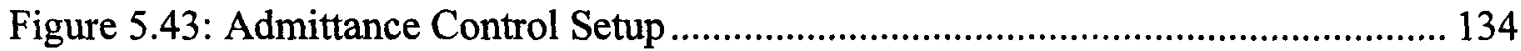

Figure 5.44: User Interface for Admittance Control Mode ...................................... 134

Figure 5.45: Information Flow of Admittance Control Mode ................................. 136

Figure 5.46: End Effector Tracking Results of Admittance Controller........................ 137

Figure 5.47: End Effector Tracking Error of Admittance Controller ........................... 138

Figure 5.48: Applied Forces during Admittance Control Experiment ......................... 138

Figure 5.49: X Position Tracking of Admittance Controller ...................................... 139

Figure 5.50: Y Position Tracking of Admittance Controller ...................................... 139 
Figure 5.51: X Position Tracking Error of Admittance Controller.................................. 140

Figure 5.52: Y Position Tracking Error of Admittance Controller.................................... 140 


\section{Chapter 1: Introduction}

\subsection{Motivation}

Every year there are approximately 10000 new cases of traumatic spinal cord injuries [1] and 795000 strokes [2] in the United States of America and 50000 in Canada [3].

Strokes are caused by either a hemorrhage or an ischemia in the brain. In either case, the condition causes decreased blood flow to a portion of the brain resulting in tissue damage and death due to hypoxia which destroys original electrical pathways in the brain [4]. As location of the tissue death varies, so do the after effects of the stroke. Some

patients have little or no after effects while other patients may die almost immediately [5]. Rehabilitation of the affected motor skills slowly enables the patient to relearn the skills lost due to the tissue death [6].

Spinal cord injuries cause damage to the nerves that form the connection between brain and the muscles. In cases where there is a complete spinal cord injury, all neural fibres are detached and no electrical impulses can be carried beyond that point; in this situation there is no voluntary motor control distal to the location of the injury. In incidents where the injury sustained to the spinal cord is partial, and there is still some function remaining below the injury, rehabilitation is a possibility [7].

A third segment of patients, with neural degenerative diseases such as Parkinson's, Multiple Sclerosis, and Amyotrophic lateral sclerosis (ALS) can benefit from rehabilitation therapy. In these cases the Central Nervous System (CNS) of the patient slowly decays. Similar to a stroke patient, it may be possible for the individual to regain mobility through rehabilitation [8][9] [10]. 


\subsubsection{Neural Plasticity}

The basis of motor rehabilitation is the concept of neural plasticity, or the ability for the brain to rewire neurons to learn new tasks based on the person's experiences. This rewiring constantly occurs in the brain, developing connections and removing synapses depending on activity [11].

Through rehabilitation, the central nervous system (CNS) adapts and transforms to the task, forming new neural pathways in the brain and spinal column. Survivors of CNS injuries often have reduced motor function and control of their bodies. In cases where the patient's mobility is impacted, individuals may be limited in their ability to perform tasks related to their occupation or, in more severe cases, their ability to perform activities of daily life (ADL), such as getting out of bed or feeding themselves [12]. The ability to perform these functions leads to a much higher quality of life. Through motor learning rehabilitation these skills can be partially or fully restored to the afflicted limbs through repetitive training. Studies of stroke rehabilitation have shown that " $58 \%$ of patients regain independence in ADL and 82\% learn how to walk" [5].

\subsubsection{Motor Learning as it Applies to Rehabilitation}

From Kleim and Jones [13], motor learning has ten specific focuses. The ten principles are listed in Table 1.1with a brief description. These ten principles can be applied to varying degrees to rehabilitation and specifically gait rehabilitation. 
Table 1.1: Ten Principles of Experience-Dependent Plasticity [13]

\begin{tabular}{ll}
\hline Principle & Description \\
\hline 1. Use it or Lose it & $\begin{array}{l}\text { Failure to drive specific brain functions can lead to functional } \\
\text { degradation } \\
\text { 2. Use it and Improve it }\end{array}$ \\
$\begin{array}{l}\text { Training that drives a specific brain function can lead to an } \\
\text { enhancement of that function } \\
\text { The nature of the training experience dictates the nature of } \\
\text { the plasticity }\end{array}$ \\
4. Repetition & $\begin{array}{l}\text { Induction of plasticity requires sufficient repetition } \\
\text { Induction of plasticity requires sufficient training intensity }\end{array}$ \\
5. Intensity & $\begin{array}{l}\text { Different forms of plasticity occur at different times during } \\
\text { training }\end{array}$ \\
6. Time & $\begin{array}{l}\text { The training experience must be sufficiently salient to induce } \\
\text { plasticity } \\
\text { 7raining-induced plasticity occurs more readily in younger } \\
\text { brains }\end{array}$ \\
8. Age & $\begin{array}{l}\text { Plasticity in response to one training experience can enhance } \\
\text { the acquisition of similar behaviours }\end{array}$ \\
9. Transference & $\begin{array}{l}\text { Plasticity in response to one experience can interfere with the } \\
\text { acquisition of other behaviours }\end{array}$ \\
\hline
\end{tabular}

\section{Use it or Lose it}

Rehabilitation that encourages use of the leg is appropriate. Whether this is through task specific training or more general rehabilitation for control of the muscles, there is an increase in functionality with an increase is usage. Once a patient has relearned a task, it is important that they continue to use this skill. Without exercise the patient could regress and no longer be able to perform the task [14]. 


\section{Use it and Improve it}

Immediately following the injury, when the patient will have little control, the process of relearning the motions requires that many intermediate steps be created. By encouraging the evolution of the training by varying the tasks and requiring the patient to focus on the task, the patient constantly improves and does not stagnate at one level of aptitude [14]. Figure 1.1 shows a representation of the brain allocation of an owl monkey from stimulation of a specific location over time. The neural area was used and due to this, a larger portion of the brain was devoted to this location, thus improved.

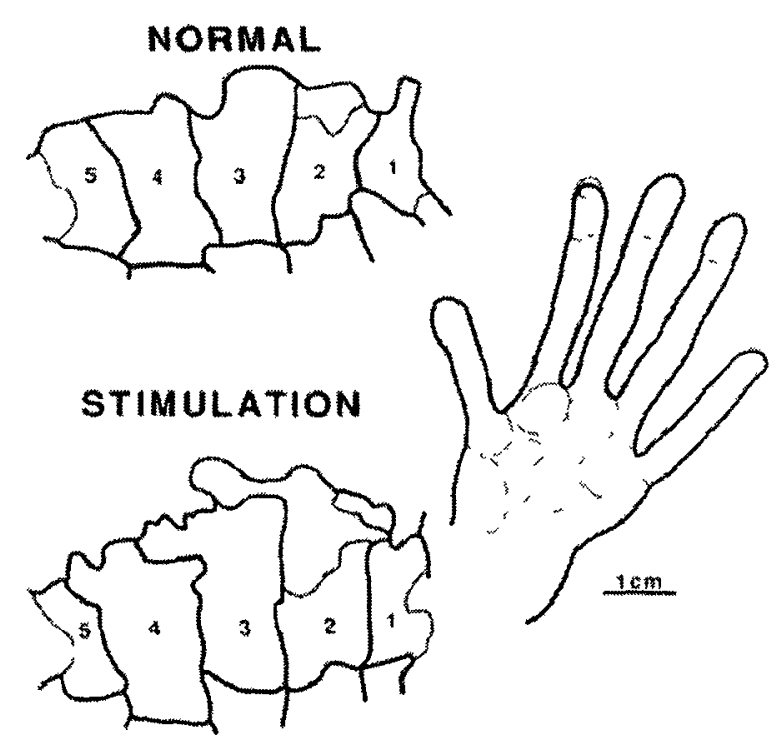

$1 \min$

Figure 1.1: Brain Surface Allocation of Normal and Stimulated Finger Use in Adult Owl Monkeys [15]

\section{Specificity}

Evaluating rehabilitation on the basis of a specific task, such as walking, a rehabilitation program that focuses on learning the skills of walking rather than more general skills will greatly decrease the recovery time and increase the performance of the patient in this task. It was found that unless tasks are almost identical, there is little transfer of the skills between tasks [14]. 


\section{Repetition}

Constant repetition of a task, such as performing the motions of walking under no or reduced load for a lower functioning patient, will aide in the reforming of the neural pathways. The focus must also be on progressing the skill set as repetition of a simple task, such as knee flexion in a patient who has little trouble performing the task, will not aide in neural plasticity [14].

\section{Intensity}

Requiring that the patient constantly exert themselves physically and mentally increases the rate at which the brain relearns the tasks. A low intensity, low level of involvement exercise where a physiotherapist is articulating a patient's knee will not have the same beneficial results as one that requires intense concentration to both a visual input of performance as well as the gait control [14].

\section{Time}

The longer a patient spends attempting to relearn a skill the more progress he/she will have. Along with a high intensity training regimen, the more time that is spent and the more rapidly a patient progresses has a dramatic impact on their final condition [14]. Also, it has been suggested that beginning rehabilitation earlier would increase the effectiveness of the training [16].

\section{Salience}

For a rehabilitation session to be salient, the patient must be occupied by performing the task at hand throughout the entire span of the session. A focus on the task aides in inducing plasticity in the brain, the less abstract a training regimen is, the more salient it will be as the patient understands the focus of the exercises and the long term goal [14]. 
Age

In rehabilitation, age is not a controllable factor. In many cases the patients that partake in physiotherapy rehabilitation sessions are elderly [14].

\section{Transference}

Rehabilitation that is focused on gait training and the development of the skills required for walking, though intended to only improve a patient's mobility can also have positive side effects from generally improving the patient's motor control. In addition to specificity, a platform that imparts general skills as well as the specific skills can be highly advantageous to the patient [14].

\section{Interference}

The inverse of transference is the possibility that the development of skills for walking could impede the learning of other skills. The learning of a specific skill could cause the plasticity of the brain to affect another function of the brain located in the similar area. Immediately following a stroke, a patient will learn how to interact with their environment however their body will allow in that state. This learning of incorrect controls could hamper the rehabilitation process of useful gait learning [14].

\subsection{Virtual Gait Rehabilitation Robot}

A novel gait rehabilitation robot, Virtual Gait Rehabilitation Robot (ViGRR), was proposed for development by the Carleton University Advanced Biomechatronics Lab $(\mathrm{ABL})$ to fill the niche of a haptic gait rehabilitation trainer which is able to articulate both in a gait trajectory as well as in the workspace of a human leg so that more complex tasks could be performed while being accessible for easy use. In order to make the system more accessible to the user a Body Weight Support (BWS) system was not used as it adds significant time to the beginning and end of the rehabilitation session. The BWS also may place the patient in a position of discomfort if they do not have the ability to support their 
own weight. From the goal of designing a platform that can impart a virtual environment to the user, a set of milestones were developed.

1) Design a prototype platform for development of control algorithms for gait rehabilitation

2) Expand prototype to full scale design, including a virtual environment with sensory feedback for the user

3) Demonstrate the platform on both healthy and CNS impaired individuals in clinical based trials

\subsection{Contributions}

This thesis presents the design and development of the ViGRR platform. The following are contributions to the project presented in this document.

\section{Development of the concept}

From the basis of designing a gait rehabilitation platform to the development of the specific design parameters developed to define the framework in which the manipulator was created.

\section{Prototype Manipulator Design and Analysis}

From the developed specifications and gait data, the design was optimized. The optimization process took into account the kinematic model of the manipulator and determined the link lengths, end effector angle, and the joint limit and singularity avoidance coefficients for the redundancy resolution scheme. The optimization process used the dynamic model to determine the joint torques and velocities and these were used to iteratively select the actuation components.

The structural components of the manipulator were designed and analyzed under an extreme loading condition of the gait cycle. The links were sized from available stock material and the joint components were designed to increase joint rigidity and decrease deflection of the end effector. 


\section{Manipulator Fabrication and Integration}

The parts of the manipulator were manufactured using Computer Numerically Controlled $(\mathrm{CNC})$ code and engineering drawings from the platform design Computer Assisted Design (CAD) files.

The drive components were purchased and integrated with the manufactured parts.

\section{Manipulator Calibration}

The assembled manipulator was integrated with the controller being developed in parallel and the manipulator was kinematically and dynamically calibrated to determine the experimental lengths of the links as well as the dynamic parameters such as the masses, locations of the link centers of gravities and inertias as well as damping and friction coefficients.

\subsection{Outline}

This section provides an overview of the contents of the remaining chapters of this thesis.

\section{Chapter 2: Evaluation of Current Technologies in Gait Rehabilitation}

This chapter presents the robotic gait rehabilitation platforms currently in development and evaluates each based on the principles presented by Kleim.

\section{Chapter 3: Platform Conceptualization and Design}

This chapter discusses the formulation of the requirements from gait data as well as the kinematics and dynamics of the manipulator and human leg. This information is then synthesized into the design and optimization of the manipulator, including dimensioning of the platform and selecting the hardware components. 


\section{Chapter 4: Design and Structural Analysis of Virtual Gait Rehabilitation Robot}

\section{Manipulator}

This chapter develops the design of the robot, the link design, material selection and joint design through iterations of finite element analysis on the manipulator.

\section{Chapter 5: Calibration and Experimentation}

In this chapter the assembled robot is kinematically and dynamically calibrated. The calibrated robot is then tested in joint space, task space and with an admittance controller to verify that the robotic design functions as intended.

\section{Chapter 6: Conclusions and Recommendations}

This chapter summarizes the work presented throughout the document and suggests possible improvements and future work. 


\section{Chapter 2: Evaluation of Current Technologies in Gait Rehabilitation}

From Kleim and Jones' ten principles presented in Table 1.1, four principles are useful to evaluate gait technologies. The four selected principles are: 1) Specificity 2)Repetition 3) Intensity 4) Salience.

This chapter outlines the various technologies currently in development for gait rehabilitation and evaluates each using four of the principles presented by Kleim and Jones. The report focuses on gait specific trainers rather than include additional support techniques used in physiotherapy sessions that focus on regaining muscle strength and control before an emphasis on gait training.

\subsection{Body Weight Supported Treadmill Training}

Body Weight Supported Treadmill Training (BWSTT) is a common method of gait rehabilitation performed on both stroke and spinal cord patients. A portion of the patient's weight is supported by an exterior harness, as in Figure 2.1, allowing for the strength of the patient to be sufficient to support themselves on top of a treadmill. It is generally accepted in the rehabilitation field that no more than $40 \%$ of a patient's body weight should be supported and that the patient should be able to support themselves for at least a short period in time before beginning vertical training. In cases where the patient has little to no control of their legs, a physiotherapist manipulates the patient's legs. In many cases, this requires multiple physiotherapists as one physiotherapist controls each leg while a third gyrates the hips corresponding to a natural gait motion[17]. 
From Kleim's ten principles, BWSTT works specifically on gait without relying on other exercises to simulate walking. There is repetition of the motion, though the length of repetition, or time, of the rehabilitation sessions is limited by the physical exhaustion of both the patient, though more likely by the physiotherapist. Additionally, multiple physiotherapists are required with at least one to articulate each leg and a third to guide the hips. Though treadmill training does an excellent job of creating the specific environment of walking on flat ground, there is no scalability to include varying terrain, such as climbing a flight of stairs or to simulate collisions or obstacles. With planar training, muscles that control turning and trajectory correction are not exercised and the fine muscle control required for balance develops slowly. The level of intensity varies depending on the specific criteria used by a therapist but any situation where the user's legs are being put through motion, regardless of whether they supply any effort is nonideal.

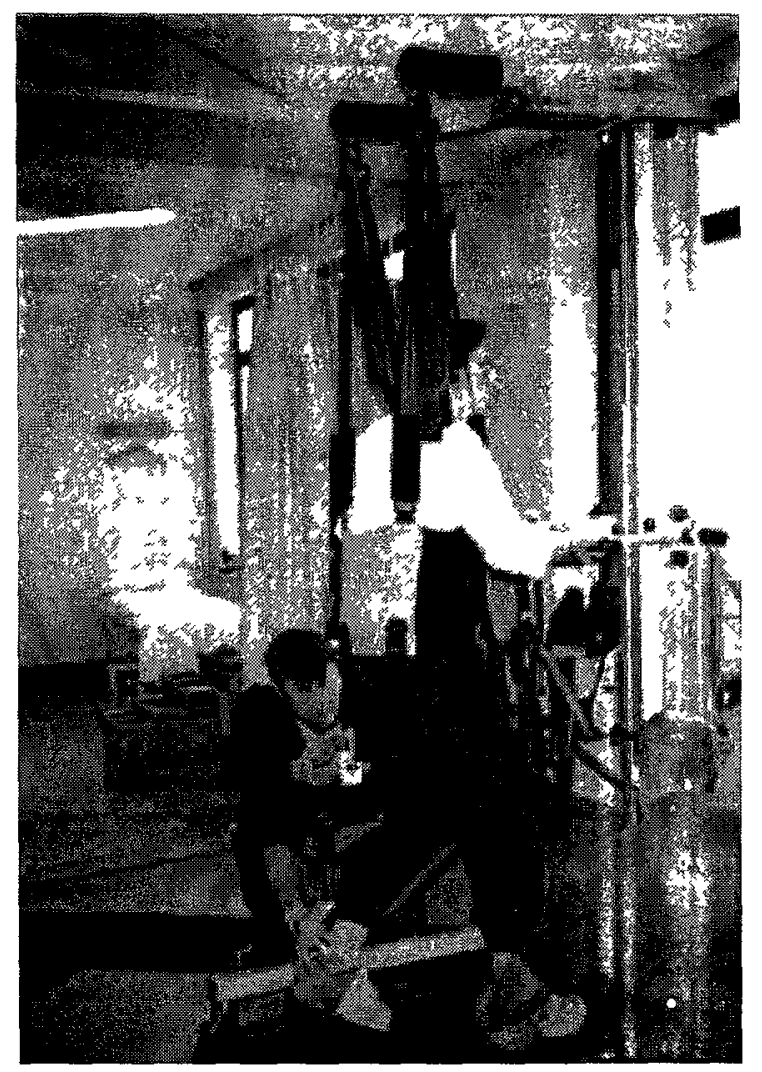

Figure 2.1: Body Weight Supported Treadmill Training [18] 
In order to address the shortcomings in traditional gait rehabilitation methods, such as BWSTT, robotic assistance is being applied to gait rehabilitation. With the use of a robotic platform, the endurance of the patient and not the therapist's stamina determines the length of the training session. The use of robotics focuses on improving all four of the selected principles from Kleim's ten principles. With the removal of the fatigue level of the physiotherapist, the repetition and time are increased as rehabilitation sessions can be longer. The control of the manipulators allows for more accurate tracking of an average gait cycle, as presented in Winter [19] and thus increased specificity. As for intensity, again, with the removal of the physiotherapist, there is no decrease in effectiveness of the rehabilitation session and the focus can be on the patient exerting themselves as much as possible. Finally, salience is addressed in many robotic platforms by supplying the patient with a visual, real time feedback of their status and allows them to concentrate on correcting their performance.

Building on the concept of BWSTT, where a treadmill is used to create a moving ground, a number of platforms have been developed to reduce the load on the physiotherapist while also attempting to improve the level of rehabilitation for the patient.

\subsection{Lokomat Rehabilitation System}

To reduce the load on the physiotherapists, the Lokomat system was developed as a position controlled treadmill based exoskeletal robotic platform Closely based on the BWSTT design, the actuators were placed alongside the patient's legs. As the platform was purely position controlled at this stage in the development, there was no information for the physiotherapist as to the level of exertion of the patient compared to the output of the robot.

Due to this lack of feedback, significant work has been performed to improve the control models and include force feedback in the system. The Lokomat system has been commercialized and has had multiple papers, [20] [21] [22] [23] [24], address the validity of the platform as a gait trainer. The original design featured four articulated joints, 
flexion and extension of both hips and knees were actuated by linear actuators. Out of plane motions were restrained including lateral translation of the hips and torso and adduction and abduction of the hip [25].

Figure 2.2 shows the updated design which contains the force feedback system. While Figure 2.3 shows the in plane design of the platform with each of the legs articulated in a planar motion. Figure 2.4 shows the actuators of the Lokomat platform.

Attempts were made to modify the platform into a haptic device with the inclusion of a toe impact model, though design constraints from the original position controlled system prevented a realistic environment [26] [27].

Studies were performed that found:

1) Initial 16 participant trial with positive results suggested larger scale testing [22].

2) Studies outlining the kinematics and joint torques of the patient while using the Lokomat trainer found that, though the pelvis and torso were fixed, there was significant movement of the user which led to the activation of muscles that normally would not be activated during normal walkıng This lack of degrees of freedom causes unnatural gait motions that require additional energy [21] [28] [29].

3) A large scale clinical trial found that the Lokomat system was inferior to traditional methods as the Lokomat system restricted the motion of the pelvis and upper body, requiring the user to exert themselves more as weight shifting was not an option. This study was performed with the original design of the Lokomat, without the force sensors or control [30]. 


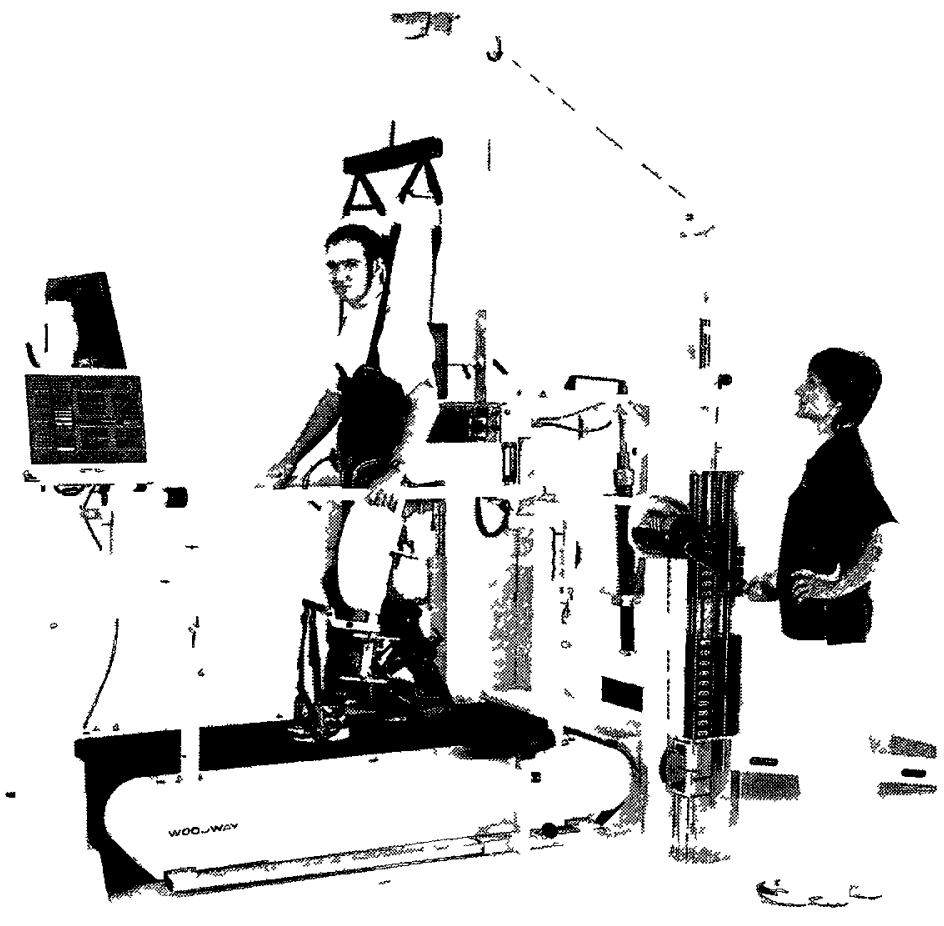

Figure 2.2: Layout of the Lokomat System [31]

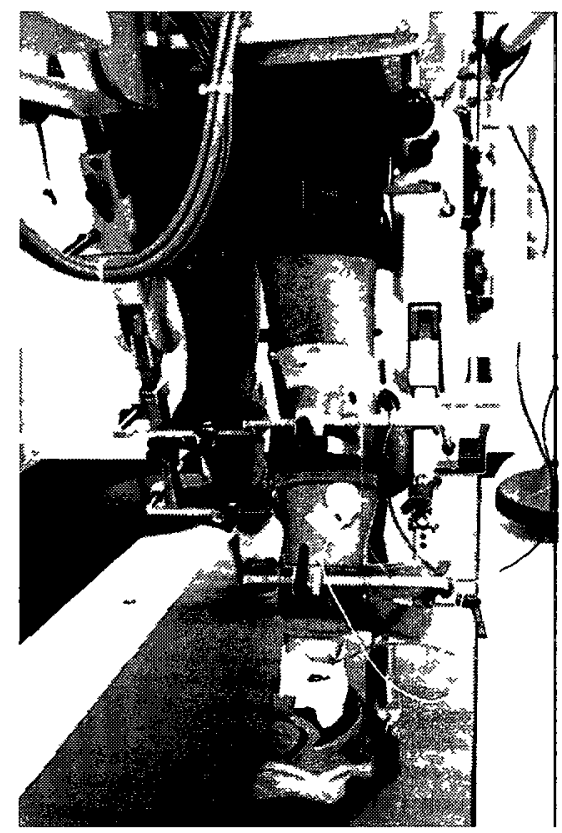

Figure 2.3: Back View of the Lokomat Gait Trainer [32] 
Evaluated on the four principles, the Lokomat addresses:

1) As the platform is focused only on gait rehabilitation the platform is highly specific.

2) The intensity of the training is dependent on the feedback to the user. The original design of the Lokomat fell short as it was run in pure position control and the user was not required to give any input. With the new control modes and Force Torque sensors the system has greatly improved the intensity.

3) Through the use of visual feedback on the progress of the rehabilitation, salience is achieved.

4) Repetition: By removing the strain on the physiotherapists, and controlling the motion with machine precision. The rehabilitation sessions can be longer and be more accurate.

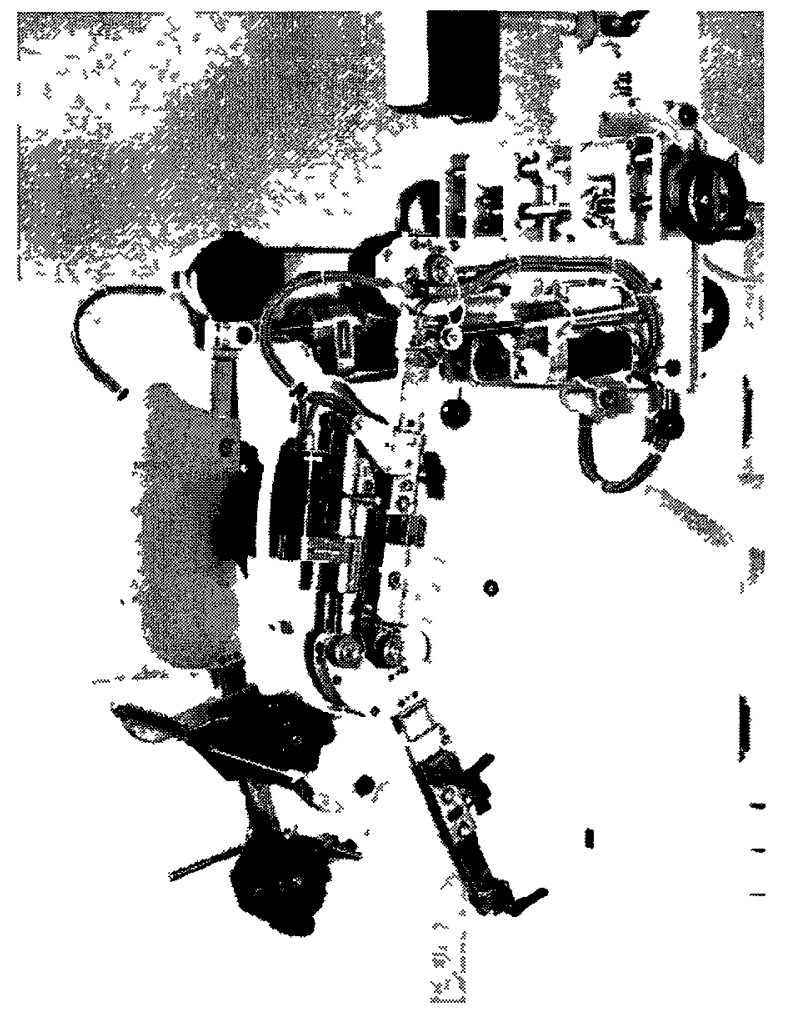

Figure 2.4: Close up of Lokomat Actuators [26] 


\subsection{LOPES}

The LOwer-extremity Powered ExoSkeleton (LOPES) is an exoskeletal, anthropomorphic robot used for treadmill training. The platform is designed to be a 'patient in charge' platform where the robot provides selective assistance as required. The robot articulates the hips, and knees, though not the ankles to assist in leg trajectories while also supporting lateral movement [33].

LOPES has eight actuated degrees of freedom and 9 free degrees of freedom, thus impeding only 5 out of all the possible degrees of freedom in the human gait, Figure 2.5. The pelvis of the patient is actuated forward and back as well as left and right. The up and down motion is freely allowed within the bounds of the system. Rotations of the pelvis are prevented and any rotations the patient does expenence is from play in the system. The hips are actuated in both in the flexion/extension and adduction/abduction degrees of freedom while the Exo/Endo rotation of the hip is fixed. The knee is articulated in the flexion/extension motion, which is the only significant degree of freedom in the human knee. The ankles are not articulated as the forward motion of the leg is assured due to the pelvic and hip motion and the contact of the foot with the treadmill is allowed to occur based on the passive joint torques of the leg. The LOPES platform is designed to attempt to allow full range of motion of the user's legs while using an exoskeleton device with limited degrees of freedom [34].

\begin{tabular}{|c|c|c|c|c|}
\hline \multirow{2}{*}{ J 1,1} & \multirow{2}{*}{$\begin{array}{l}\text { Human } \\
\text { pomblubly }\end{array}$} & \multicolumn{3}{|c|}{ Rolkot dengen } \\
\hline & & Actualed & 1 res & Hhoked \\
\hline $\begin{array}{l}\text { Polionto } \\
\text { lived } \\
\text { mothl }\end{array}$ & $\begin{array}{l}3 \text { rotalums } \\
\text { 3tatslatwots }\end{array}$ & 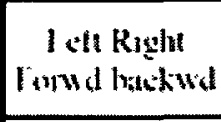 & lpdown & $\begin{array}{l}\text { All rolation } \\
\text { except lin plas }\end{array}$ \\
\hline 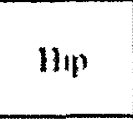 & is attatcoms & 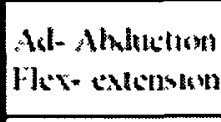 & - & 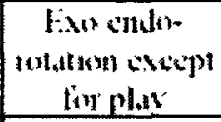 \\
\hline Knet & $\begin{array}{l}1 \text { rotatuon } \\
\text { (wagltul) }\end{array}$ & flex- extctovon & Hu- Hadtatum & - \\
\hline Ankle & 3 jotatum: & - & All muthens & . \\
\hline
\end{tabular}

Figure 2.5: LOPES Degrees of Freedom [34] 
To determine the level of resistance the constrained degrees of freedom of the LOPES system apply, a study comparing the gait cycle with and without the device was performed. In comparing the kinematics and gait parameters it was found that there were no discernable differences in the gait parameters while the kinematic model of the assisted gait had reduced flexion of the knee [35]. Figure 2.6 depicts the degrees of freedom for the one actuated leg as well as the pelvic degrees of freedom.

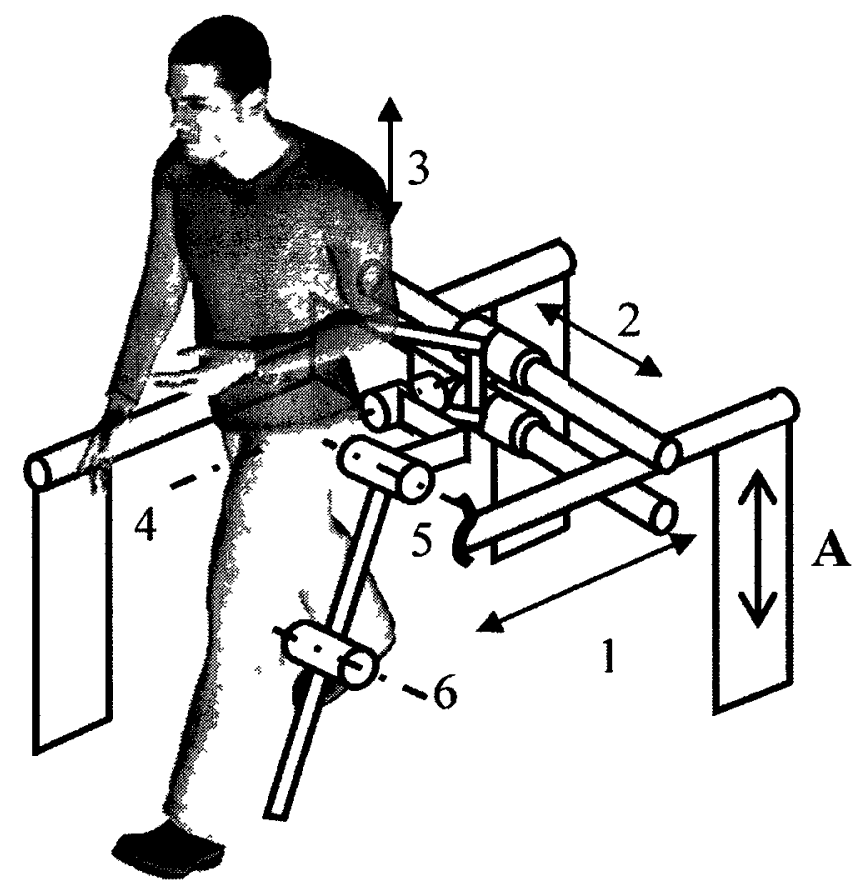

Figure 2.6: Layout of the Degrees of Freedom of the LOPES system [34]

The LOPES platform uses cable driven joints so that the actuators can be placed on the support frame, reducing the mass of the manipulator links, in Figure 2.7. The LOPES system is an impedance based platform which aides as needed throughout the gait cycle, rather than the patient following a set trajectory. 


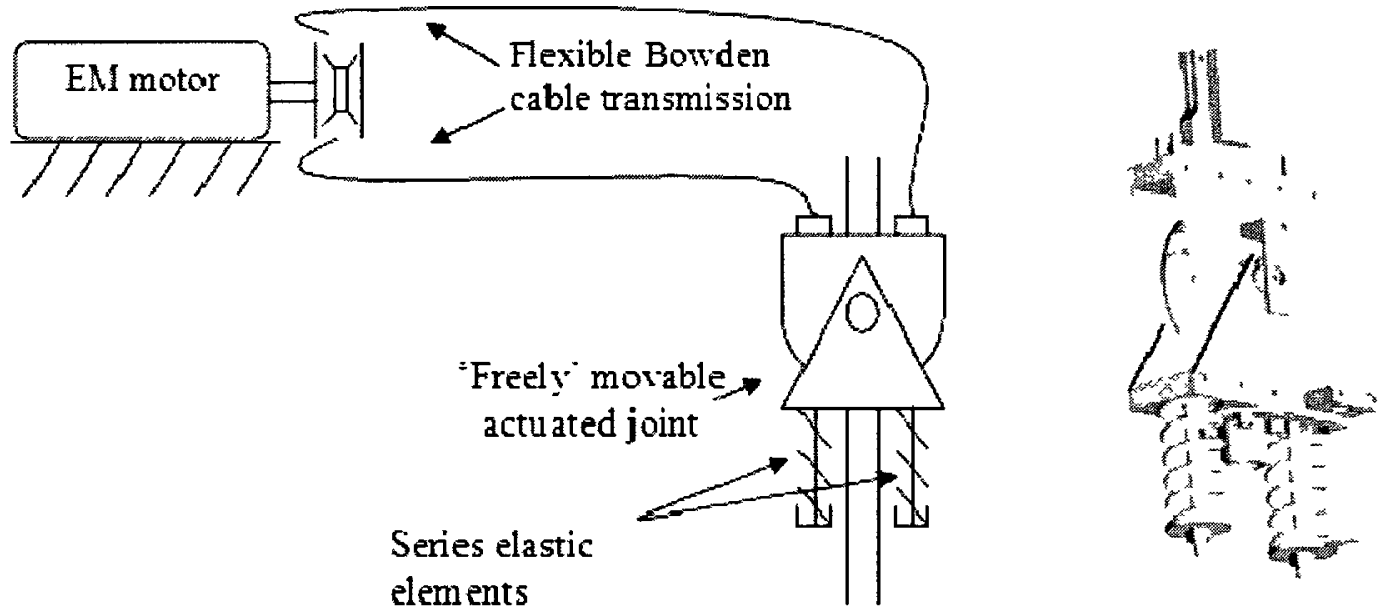

Figure 2.7: Design of the LOPES Knee joint articulator [34]

Based on Kleim's four Principles, the LOPES system performs well.

1) Specificity - Due to the large amount of flexibility in the system from the degrees of freedom, the LOPES system emulates natural gait very closely.

2) Repetition - With the removal of the physiotherapist from the process, like the Lokomat system, the trajectory planning becomes highly repetitive.

3) Intensity - By allowing the natural gait motion of the patient the platform allows the patient to focus on learning the correct motions to relearn an effectıve gait.

4) Salience - No current development of additional feedback for the user was mentioned in the literature, with the addition of a form of immediate feedback, this patient in charge device is highly flexible and represents a well designed treadmill gait trainer.

\subsection{ALEX}

The Active Leg EXoskeleton (ALEX), is a treadmill based gait trainer. Figure 2.8 depicts the various degrees of freedom of the robot. $\mathrm{A}$ is the base of the platform which travels alongside the treadmill on a wheeled chassis. B is a parallelogram mechanism for vertical translation, $\mathrm{C}$ is a plate for horizontal translation in the frontal plane. Rotary joint 
D supports abduction and adduction of the hips and $\mathrm{E}$ is a rotary joint for sagittal motion. $\mathrm{F}$ is the treadmill base. Rotation about $\mathrm{G}$ allows the patient to twist their torso. $\mathrm{H}$ is a back support, $\mathrm{I}$ is strapping for the pelvis, $\mathrm{J}$ is a thigh brace, $\mathrm{K}$ is the articulated knee joint and $\mathrm{L}$ is the shoe insert. Figure 2.9 shows the platform in use by a patient, with visual feedback provided to the patient via the computer screen on the wall.

Similar to the LOPES system, ALEX adds rotation of the trunk to facilitate shifting of body weight into the manipulator and operates with a force feedback impedance control that corrects the patient's motion outside of predefined bounds.

Kleim's four principles here are similar to the results of LOPES.

1) With the added rotation of the torso on top of the motions of the LOPES structure the ALEX platform closely mimics the motions of natural gait. Though as a treadmill based platform the design is only able to provide rehabilitation of walking on level ground.

2) Moving in the natural motion of gatt allows the patient to more consistently learn the proper body motions and controls for effective gait.

3) and 4) As the platform has force feedback into the system and visual feedback for the patient, the system allows for an intense and salient rehabilitation.

\subsection{MotionMaker}

The MotionMaker device, Figure 2.10, is an exoskeleton controlled by functional electrical stimulation (FES) from the user. The design of the platform focuses more on spinal cord injury patients rather than those suffering from the after effects of a stroke. This allows the platform to be used by paraplegics to combat health complications from underuse of their legs, such as the formation of blood clots. The use of the FES also helps with the development of voluntary muscle activity in the patıent. Linear actuators are used to control the hip, knee and ankle, Figure 2.11 shows the design of the knee joint. A five patient study was performed and shows promising preliminary results in usability of the device [36]. 


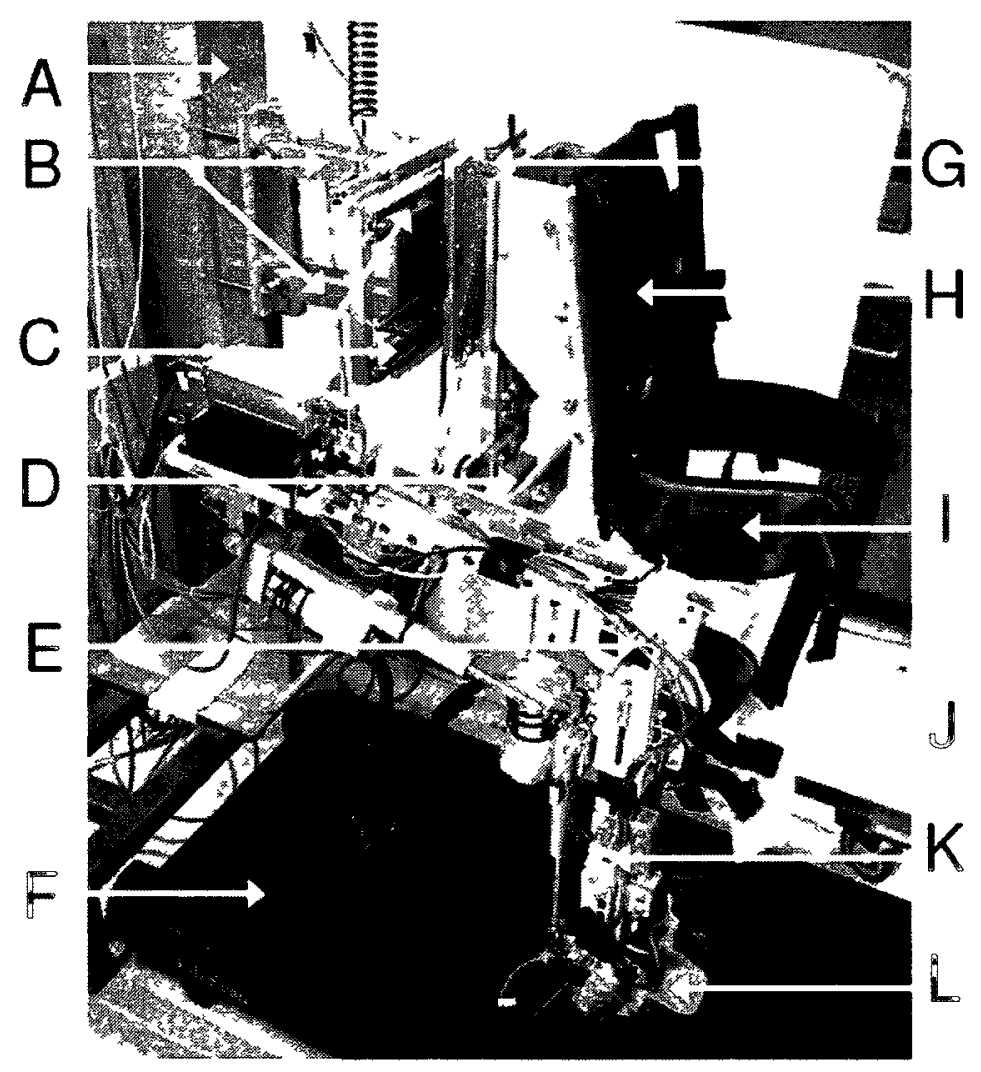

Figure 2.8: ALEX Rehabilitation Device [37]

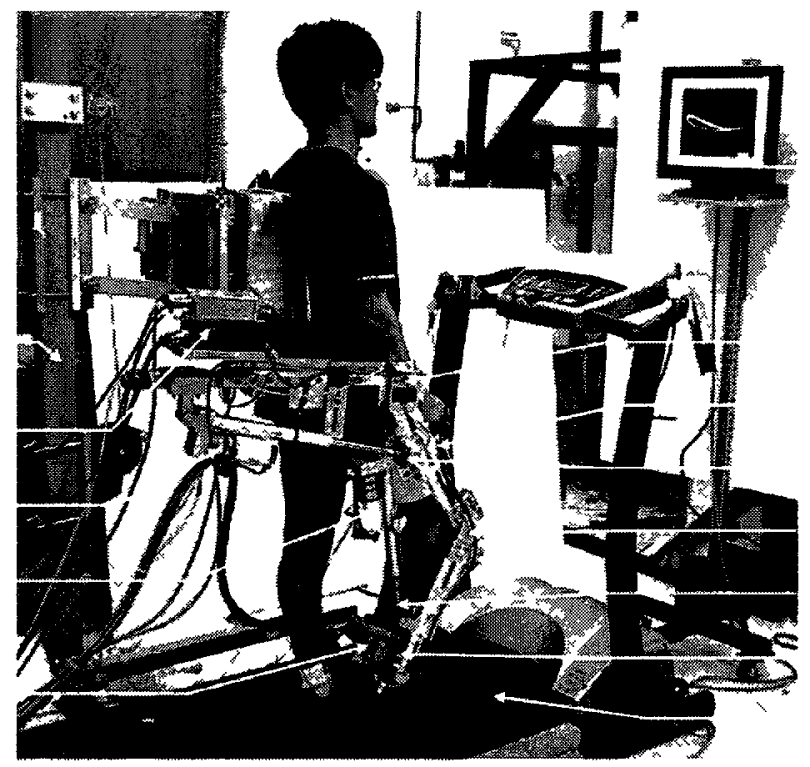

Figure 2.9: ALEX System in Use with Visual Feedback [37] 


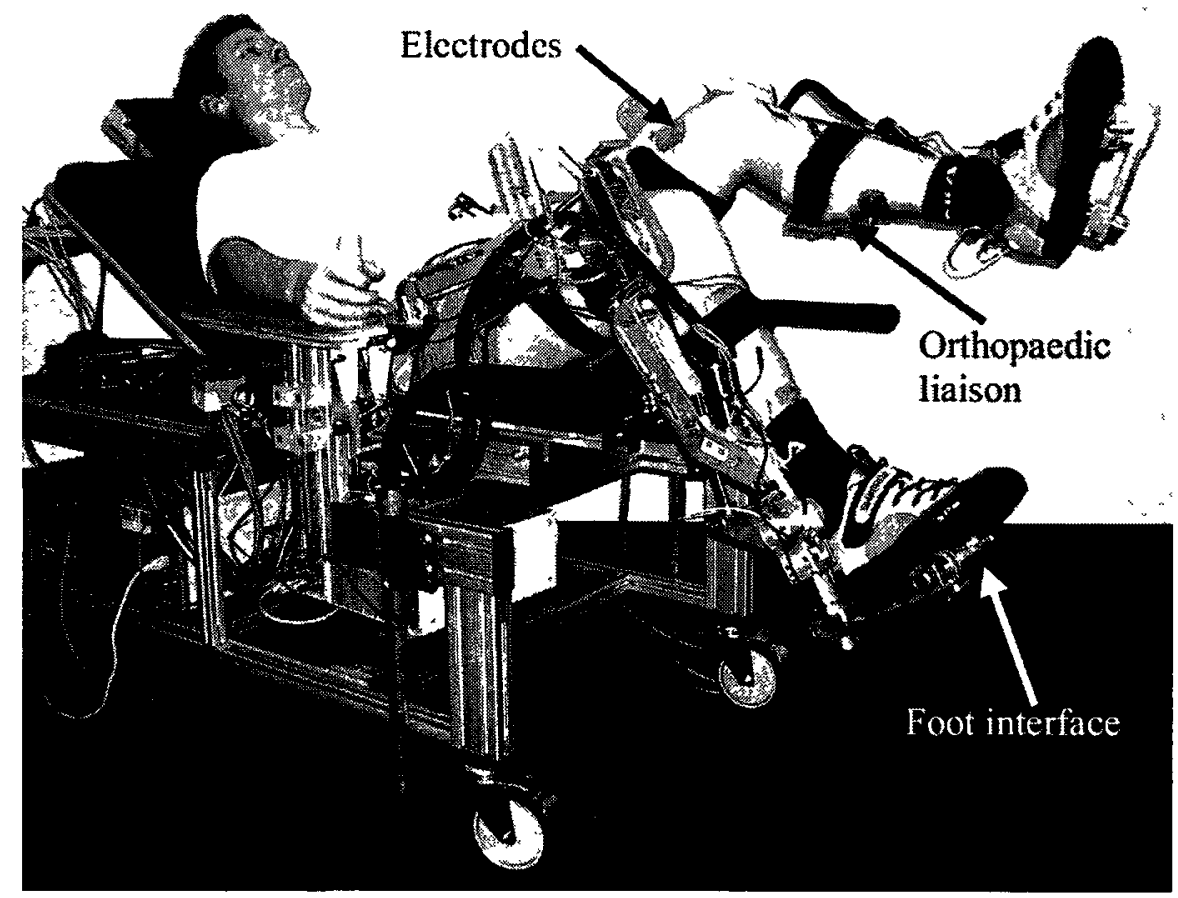

Figure 2.10: MotionMaker Platform with FES Driven Actuation [36]

From Kleim's principles:

1) Specificity - The platform focuses specifically on leg motion, though it mainly focuses on general motion or a position control scheme. As the device is also planar and anthropomorphic, major restrictions are put on the natural motion of the leg, leading to exercises that may not be relatable to walking.

2) Repetition - Under a position control scheme the repetition of the motion is very high.

3) Intensity - In cases where there is no sensory feedback into the system about the state of the patient, the level of intensity is drastically reduced.

4) Salience - Minimal due to the fact the system is primarily position control driven. Under FES control there would be high salience as a direct neuro electrical signal creates the movement. 


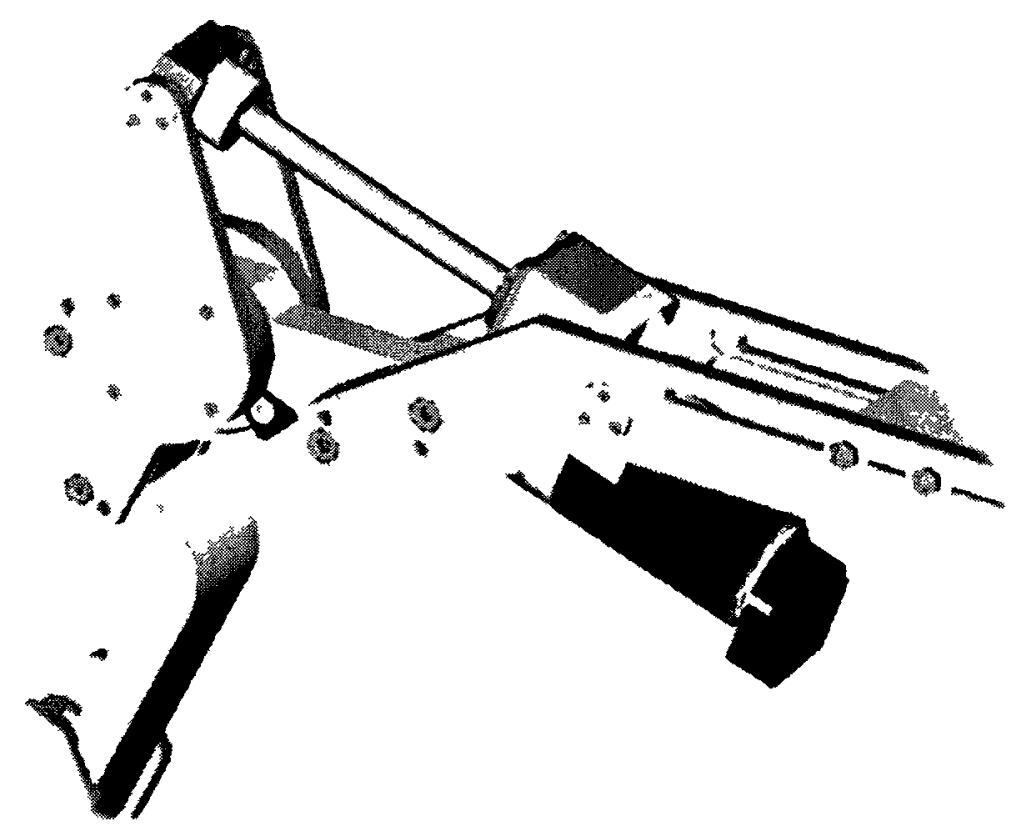

Figure 2.11: Design of MotionMaker Knee Joint with Servo Motor Powered Ball Screw Actuator [36]

\subsection{WalkTrainer}

The WalkTrainer was developed using the skills gained from the construction of the MotionMaker. The patient is suspended from a body weight support harness and FES is used to activate the platform and navigate, Figure 2.12 and Figure 2.13. The pelvis is supported using a six degree of freedom design with 6 actuators [38]. This actuation of the hips along with a leg orthosis guides the leg in a gait cycle. 


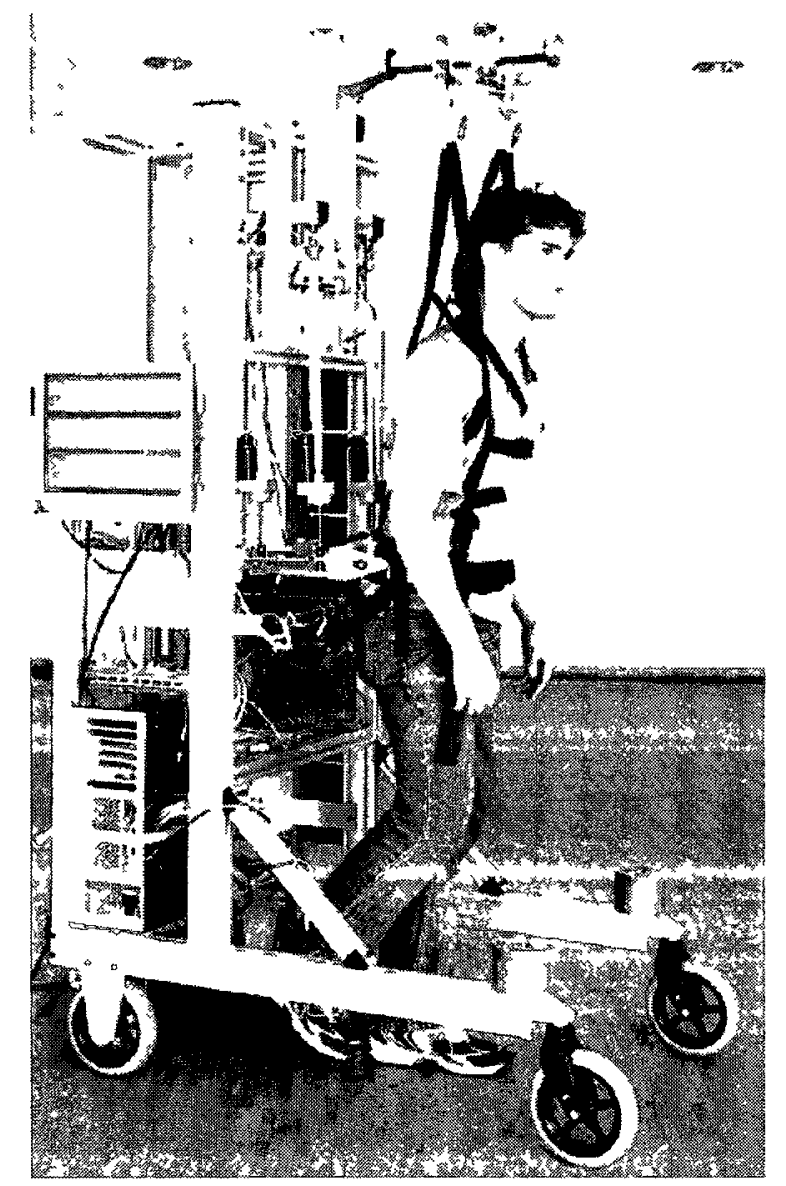

Figure 2.12: WalkTrainer in use by Healthy participant $\llbracket 38 \rrbracket$

Using Kleim's principles to evaluate the WalkTrainer

1) By allowing the patient to leave the treadmill and work on more aspects of gait, such as turning, the patient has the opportunity to learn skills that are important for navigating their environments.

2) Moving in the natural motion of gait allows the patient to more consistently learn the proper body motions and controls for effective gait.

3) By allowing a larger flexibility for the patient to explore a wider degree of movements, intense concentration as well intense muscle response is required.

4) Through navigation of their environment the salience of the experience is self evident. 


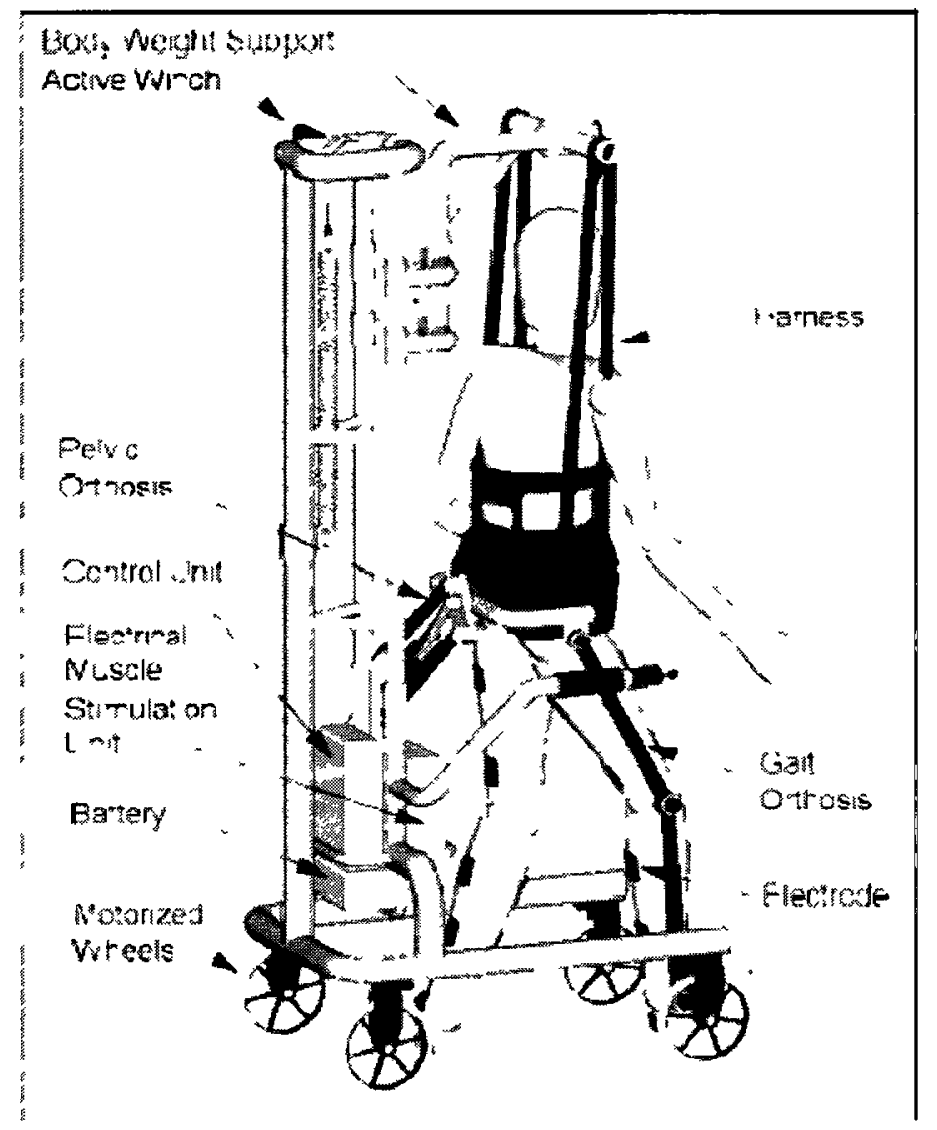

Figure 2.13: Layout of WalkTrainer Mobile Platform [38]

\subsection{Haptic Walker}

The Haptic Walker platform, Figure 2.14 is a haptic end effector controlled platform. While the Lokomat, LOPES and ALEX platforms approach the rehabilitation problem by adapting the treadmill training platform in different methods. The Haptic Walker creates a virtual environment where the user's feet are attached at all times to the manipulators of the platform and manipulates the legs by allowing them to follow the foot. The patient's mass is supported by a harness either as a safety feature, or to relieve the patient of load through body weight support. 


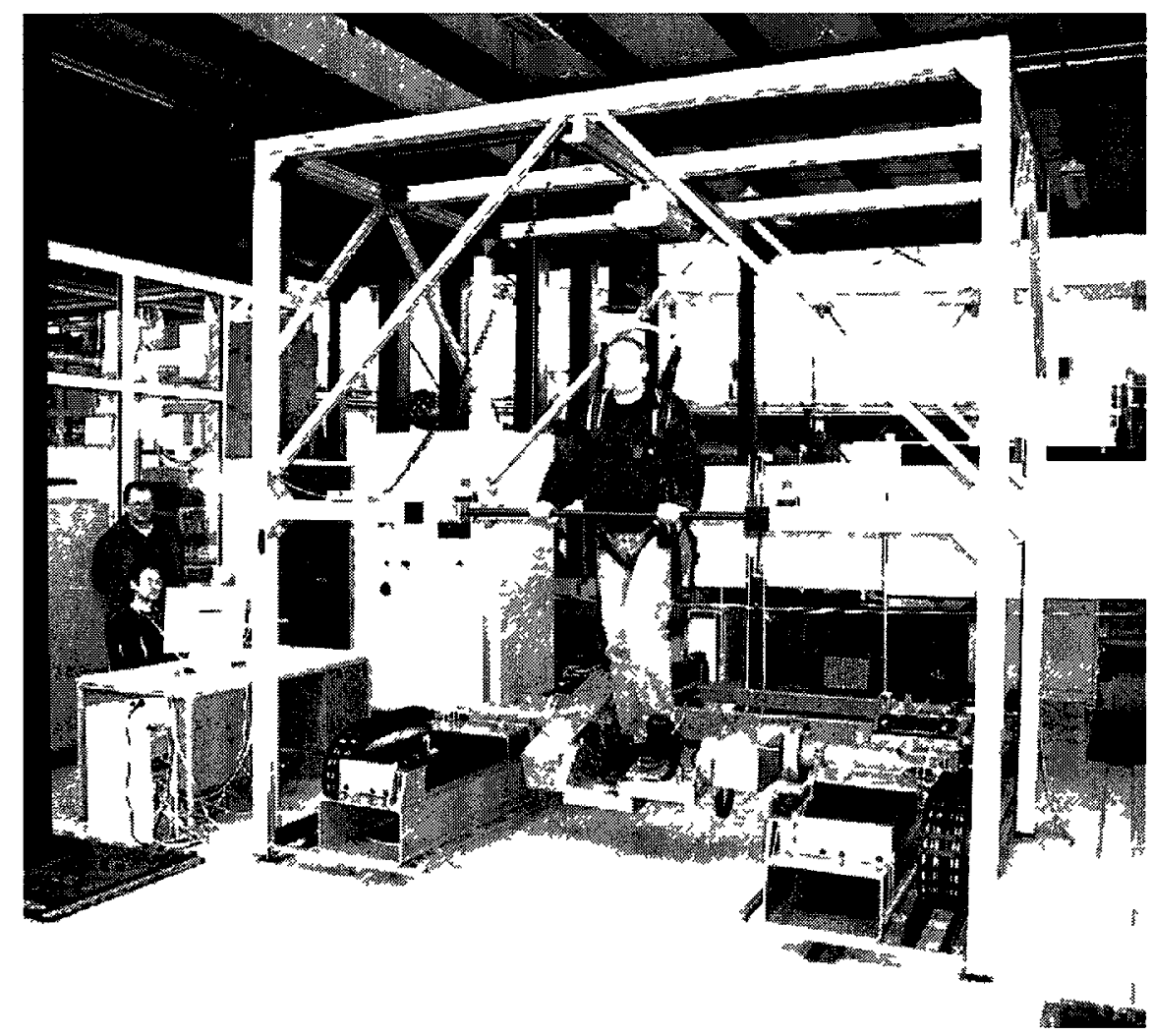

Figure 2.14: Haptic Walker in Use [39]

In comparison to treadmill training where it is difficult to emulate any environment other than walking on flat ground, though an incline can easily be applied by tilting the platform, the Haptic Walker allows the patient to interact with a variety of situations, such as climbing or descending stairs or collisions with an obstacle. Although the Haptic Walker emulates the ground and articulates the foot through the gait trajectory, there is no assistance for the motion of the knees or hips, though this in turn does not inhibit the degrees of freedom of the body. The design of the attachment solely at the foot reduces the amount of tıme required for set up and at the end of the rehabilitation sessions.

Due to the strength of the actuators used for the haptic platform, a focus was put on the safe attachment of the foot to the platform, as well as other safety features. Figure 2.15 shows the design of the foot attachment including the safety releases, joint limit constraints and the force torque sensor. 


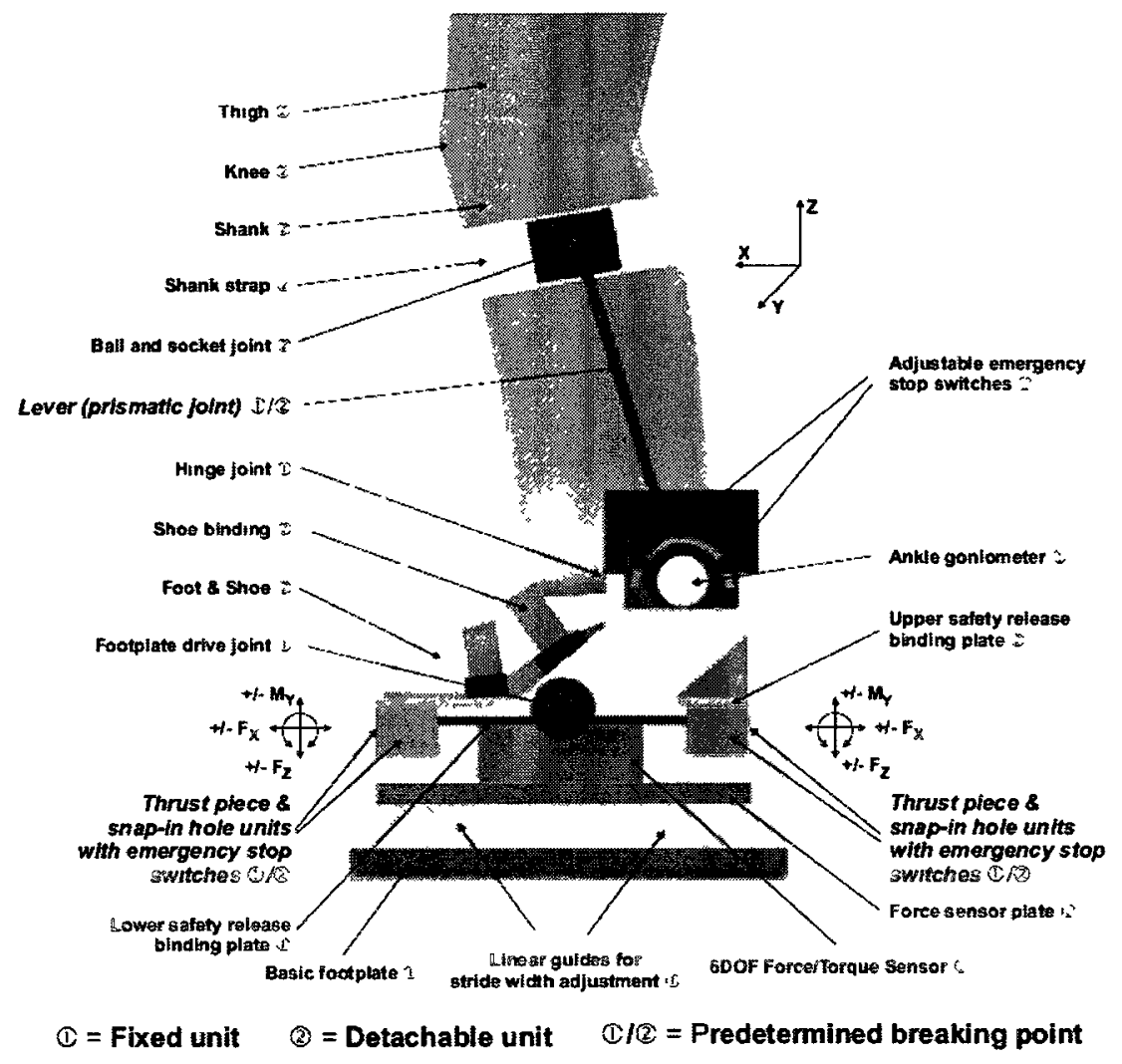

Figure 2.15: Haptic Walker Footplate Design [39]

From Kleim's ten principles:

1) Actuating only the foot of the patient and allowing the rest of the leg to follow allows for the actuation of fewer degrees of freedom as you are actuating in task space while not inhibiting motions of the joints. This all leads to a highly specific gait training.

2) As with all the other gait rehabilitation platforms, by allowing a robotic controller to determine the desired trajectory, the efficiency of the training increases accordingly.

3) Under force control the user must impart load into the system in order to actuate the end effectors. By setting up a controller to take advantage of the force feedback, the user will be intently focused on the task at hand. 
4) The haptic design is to accurately impart a virtual world to the patient through force interaction. By doing this correctly and through the inclusion of a visual feedback for the patient the system can be very salient.

\subsection{G-EO Systems}

The G-EO, Figure 2.16, is an end effector based platform that the patient is supported in by a BWS harness. The platform performs both level ground walking as well as stair climbing and descending. A graphical user interface feeds the information back to the user and gives them an assessment of their progress to aid in improving their skills on the fly. A study of the feasibility of the controller found that for a patient currently undergoing rehabilitation the platform represents an evolution from the Haptic Walker design and has added functionality over treadmill gait based controllers [40].

Figure 2.17 shows the actuation components of the G-EO platform. In the figure, item 1 is the principle drive, 2, the relative drive. 3) is the actuator for the foot angle control and 4 is the principle sledge, which is the primary foot in the controller, and 5 the secondary, relative, sledge. Item 6 is the linear guide and 7 is the screw axle [41].

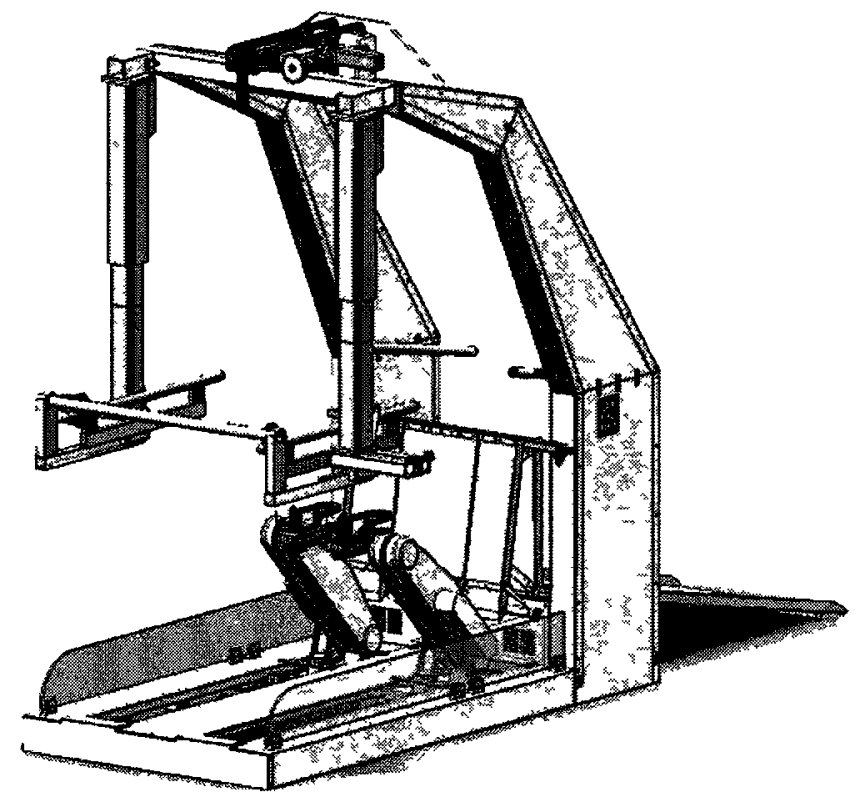

Figure 2.16: G-EO Gait Motion Device [41] 


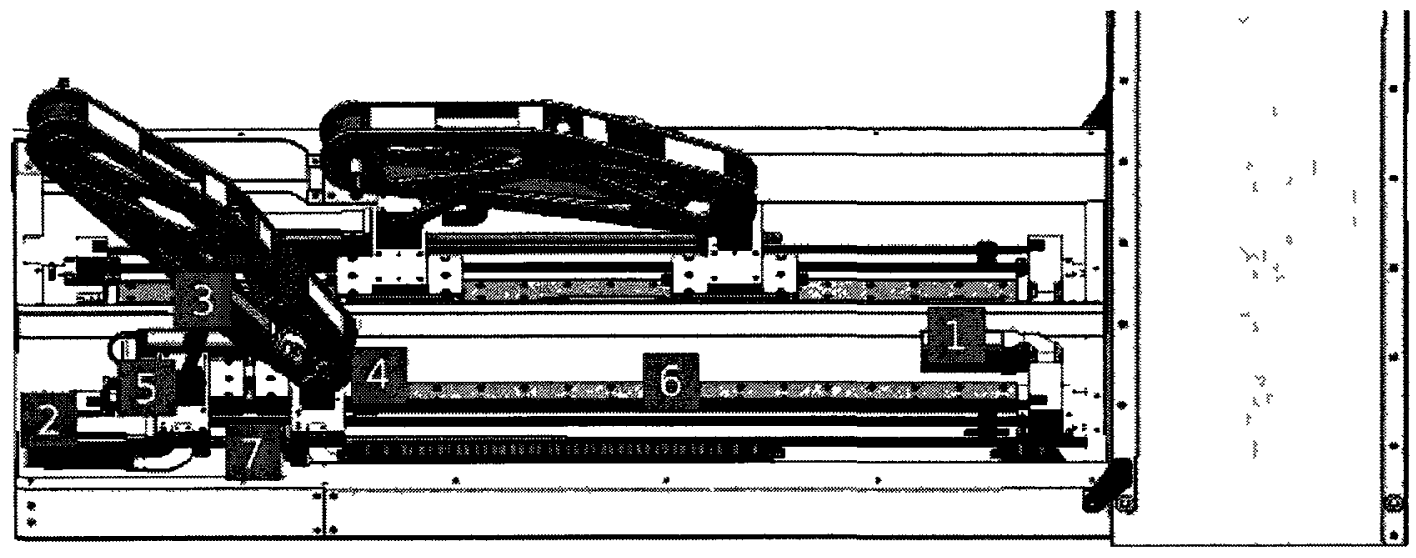

Figure 2.17: Actuation of G-EO Platform [41]

1) Specificity - Provides an end effector platform for both gait and stair training which allows for very specific controls of the trajectory and allows the physiotherapist to work on multiple highly critical components of gait.

2) Repetition - As force feedback has not yet been implemented on the platform the repetition is high but the intensity and saliency drop off as well.

3) Intensity - Low, currently no force feedback implemented in the system.

4) Salience - Low, currently no force or visual feedback implemented in the system.

As the G-EO System is being developed and expanded on, it will become a more complete system and though it currently does not meet the intensity and salience levels of many of the other platforms, the project plans include designs to address both of these.

\subsection{Results}

Extracting the conclusions drawn in each section and tabulating the results gives an overview of the current state of the platforms in development. Table 2.1 shows the tabulated results. 
Table 2.1: Evaluation of Gait Trainers

\begin{tabular}{|c|c|c|c|c|c|}
\hline & Type & Specificity & Repetition & Intensity & Salience \\
\hline BWSTT & Treadmill & Med & Med & Med & Med \\
\hline Lokomat & Treadmill & Med & High & Med & Med \\
\hline LoPES & Treadmill & High & High & High & Med \\
\hline ALEX & $\begin{array}{l}\text { Treadmill/ } \\
\text { Overground }\end{array}$ & High & High & High & High \\
\hline MotionMaker & Reclined & Low & High & Low & Low \\
\hline WalkTrainer & $\begin{array}{l}\text { Overground } \\
\text { /Treadmill }\end{array}$ & High & High & Med & High \\
\hline $\begin{array}{l}\text { Haptic } \\
\text { Walker }\end{array}$ & $\begin{array}{l}\text { Haptic } \\
\text { General }\end{array}$ & Very High & High & High & High \\
\hline $\begin{array}{l}\text { G-EO } \\
\text { System }\end{array}$ & Haptic Gait & Med & High & Low & Low \\
\hline
\end{tabular}

Though evidently very similar to one another when compared empirically like this, each design has its advantages and disadvantages. The treadmill based robots are limited in their applications as they rely on a flat, even ground and thus cannot train the patient for either rough terrain, or stairs. The MotionMaker platform is primarily a motion generator for patients with spinal cord damage. As such, it does not have a feedback capacity for the patient, as the FES only gives an output and there is no secondary evaluation.

The Haptic Walker, this platform is able to impart accurate ground reaction forces to the patient as well as simulate various environments such as stairs. The concern with the current Haptic Walker platform is that it is very large and has a very large electricity draw, the next iteration of the Haptic Walker design, the G-EO System, has been 
downsized to take 240VAC. Lastly the G-EO system, though haptic and able to create a walking and starr climbing environment, is limited to work within this range and not operate within the larger range of the human leg.

\subsection{Summary}

Using Kleim's 10 principles of motor learning, and extracting the four that most apply to gait rehabilitation, the various gait training platforms that are currently in development were evaluated. The ViGRR platform is developed to fit within these principles and to assist patients in their gait rehabilitation. 


\section{Chapter 3: Platform Conceptualization and Design}

Immediately following an injury to the CNS, a patient is started on a regimen of physiotherapy. A reclined position platform will allow for patients to begin robot assisted rehabilitation sooner as they can begin exercising in the device immediately following the injury. As shown in the previous chapter, multtple robotic approaches are being pursued in order to develop systems that will simulate a walking environment and to allow patients to relearn how to navigate their world.

The principle design requirement for this platform is a device able to manipulate the patient's feet through a gait trajectory with a cadence of 100 strides per minute, a normal walking speed. Additionally, a low cost and minimally invasive design was favoured. From this goal a clear set of requirements was defined.

Though different literature presents varying values for the cadence of an adult human, Drillis: 112, Du Catinier: 116, Finley and Cody: 110, a cadence of 100 strides per minute was selected as the design point for the platform. This is 50 strides per leg per minute or a 1.2 second period.

This chapter covers the formulation of the design problem based on gait analysis data and presents the requirements and features of the ViGRR platform. From these requirements the design of the robot was optimized based on the kinematic and dynamic model of the patient and manipulator. Included in the optimization was the selection of the hardware components for the manipulator. Figure 3.1 details the design process and iterations performed throughout the preliminary design process. 


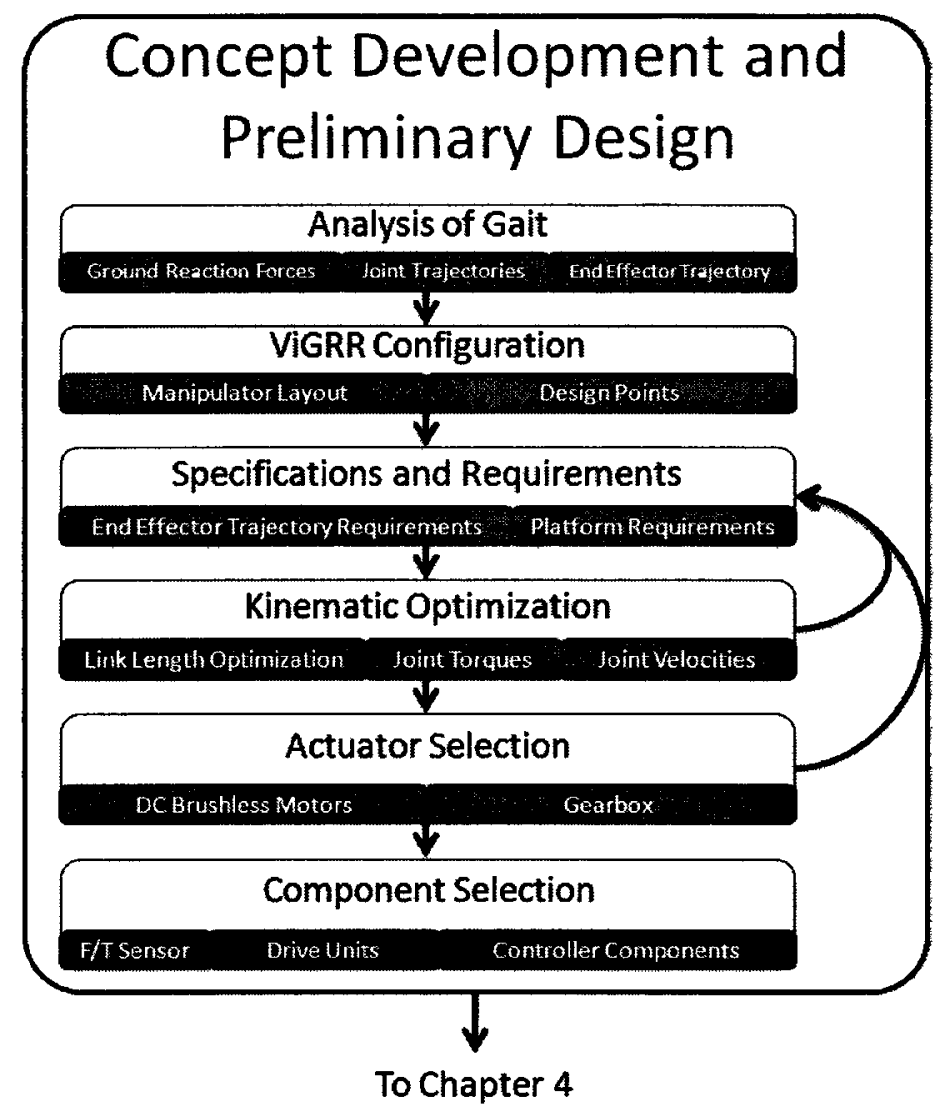

Figure 3.1 Chapter 3 Design Flow

\subsection{Gait Analysis}

In order to determine the requirements of the robot, including the work envelope and end effector loading, an analysis of human gait was required. The average gait speed is 100 strides a minute, or 50 steps per leg with a cycle time of 1.2 seconds. Winter [19] presents the data in three classifications, slow, normal and fast walking and data is presented by percentage of the gait cycle.

The leg is approximated as a three degree of freedom linkage for gait analysis as there are only very small out of plane motions. There are two phases of the gait cycle: the "stance phase", which is the time where the foot is in contact with the ground and the "swing phase", when the foot is swinging back to the heel strike. The interaction between the person and the ground creates large compression loads on the leg as well as shear 
forces to propel themselves forward. Figure 3.2 and Figure 3.3 show the ground reaction forces normalized by mass for an average speed gait. In Figure 3.2, the solid black line represents the user's mass. In Figure 3.3, the vertical axis represents the horizontal ground reaction force (GRF), positive values represent anterior shear during toe off, while the negative values represent the posterior shear during heel strike.

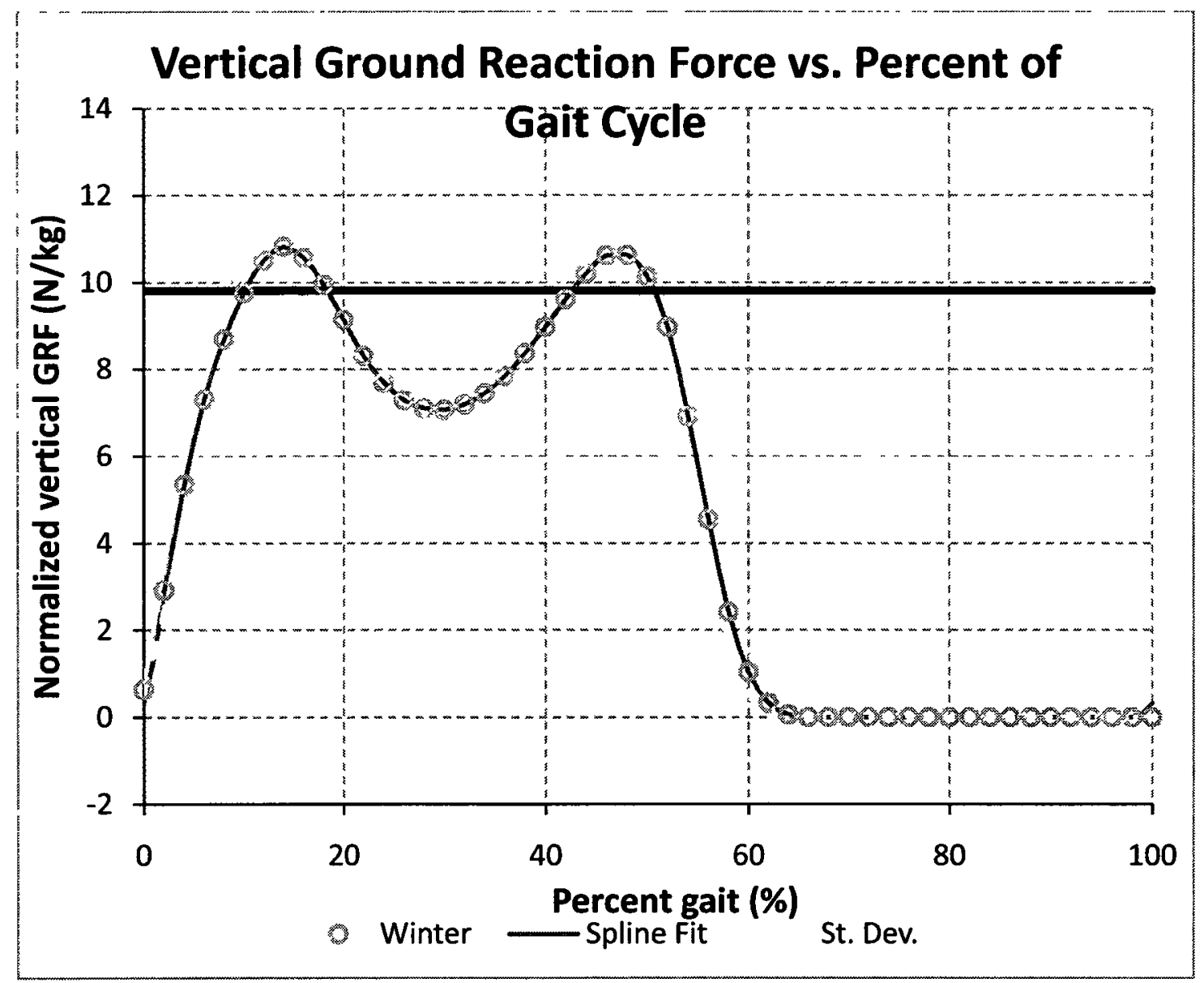

Figure 3.2: Vertical Ground Reaction Force vs. Percent of Gait Cycle 


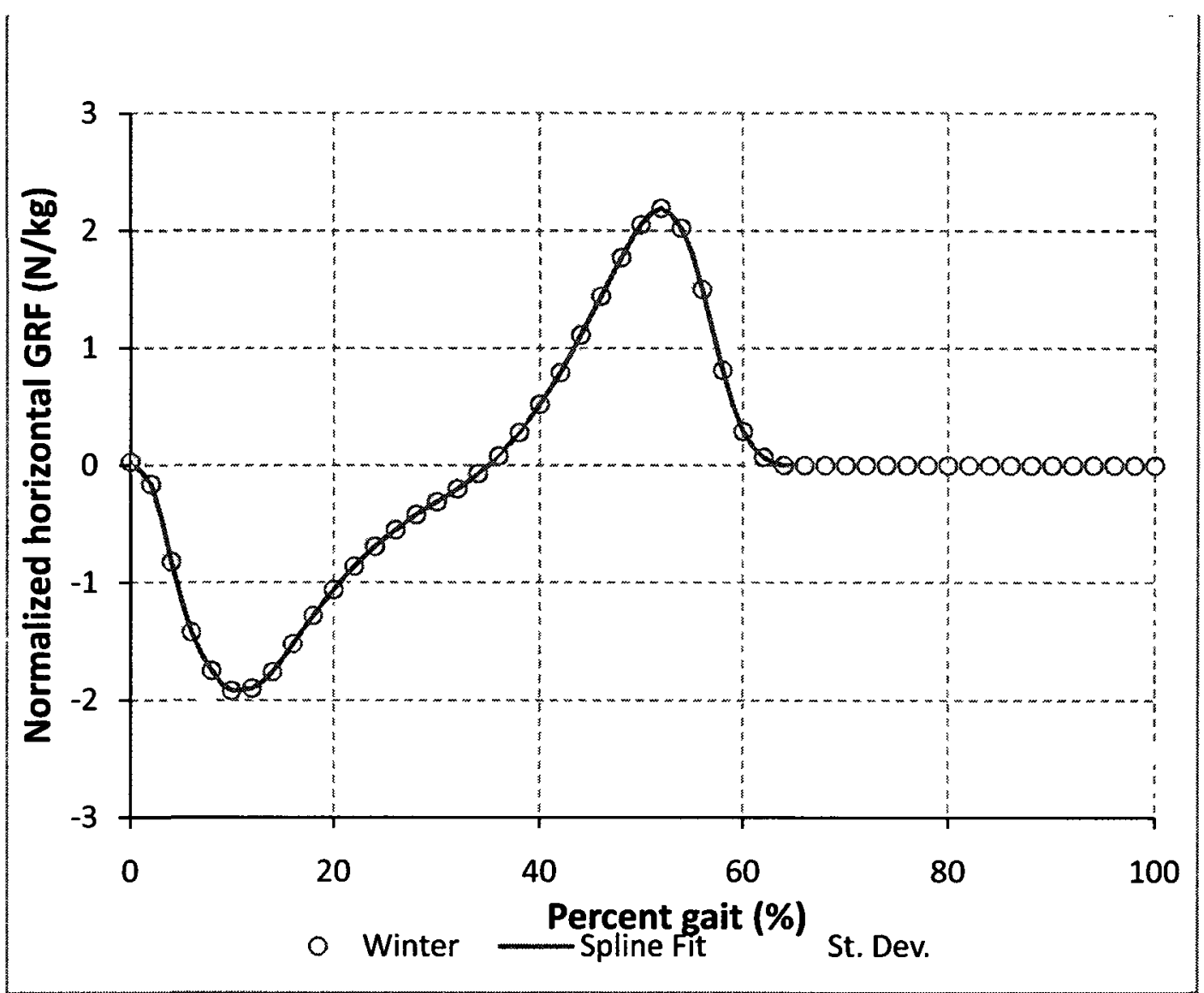

Figure 3.3: Horizontal Ground Reaction Force vs. Percent of Gait Cycle

Data provided by Winter [19] detailing joint angles, velocities and accelerations by percent of a stride, as shown in Figure 3.4 through Figure 3.6, was used in combination with the data shown in, Table 3.1, collected from McDowell [42] and Dempster [43] for a large male $\left(95^{\text {th }}\right.$ percentile) and small female $\left(5^{\text {th }}\right.$ percentile) to determine the trajectory of the end effector/foot for a gait cycle with the hip joint remaining stationary and allowed only to rotate. The normalized joint moments for the hip, knee and ankle are shown in Figure 3.7 through Figure 3.9. 
Table 3.1: Statistical Patient Dimensions

\begin{tabular}{|c|c|c|c|}
\hline & $\begin{array}{l}\text { Ratio to } \\
\text { Height or } \\
\text { Weight }\end{array}$ & $\begin{array}{c}95^{\text {th }} \\
\text { Percentile } \\
\text { Male }\end{array}$ & $\begin{array}{c}5^{\text {th }} \\
\text { Percentile } \\
\text { Female }\end{array}$ \\
\hline Height (m) & & 1.887 & 1.507 \\
\hline Mass (kg) & & 88.3 & 74.7 \\
\hline $\begin{array}{r}\text { Length of Thigh } \\
(\mathrm{m})\end{array}$ & $\begin{array}{l}0.245 \mathrm{x} \\
\text { Height }\end{array}$ & 0.462 & 0.369 \\
\hline Mass of Thigh $(\mathrm{kg})$ & $0.100 \times$ Mass & 8.830 & 7.470 \\
\hline $\begin{array}{r}\text { Center of Mass of } \\
\text { Thigh }(\mathrm{m})\end{array}$ & $\begin{array}{l}0.433 \mathrm{x} \\
\text { Length of } \\
\text { Thigh }\end{array}$ & 0.200 & 0.160 \\
\hline $\begin{array}{r}\text { Length of Lower } \\
\text { Leg (m) }\end{array}$ & $\begin{array}{l}0.246 \mathrm{x} \\
\text { Height }\end{array}$ & 0.464 & 0.371 \\
\hline $\begin{array}{r}\text { Mass of Lower Leg } \\
(\mathrm{kg})\end{array}$ & $\begin{array}{c}0.0465 \mathrm{x} \\
\text { Mass }\end{array}$ & 4.106 & 3.474 \\
\hline $\begin{array}{r}\text { Center of Mass of } \\
\text { Lower Leg (m) }\end{array}$ & $\begin{array}{c}0.433 \mathrm{x} \\
\text { Length of } \\
\text { Lower Leg }\end{array}$ & 0.201 & 0.161 \\
\hline Length of Foot (m) & $\begin{array}{l}0.074 \mathrm{x} \\
\text { Height }\end{array}$ & 0.140 & 0.112 \\
\hline Mass of Foot $(\mathrm{kg})$ & $\begin{array}{c}0.0145 \mathrm{x} \\
\text { Mass }\end{array}$ & 1.280 & 1.083 \\
\hline $\begin{array}{r}\text { Center of Mass of } \\
\text { Foot }(\mathrm{m})\end{array}$ & $\begin{array}{l}0.249 \mathrm{x} \\
\text { Length of } \\
\text { Foot }\end{array}$ & 0.035 & 0.028 \\
\hline
\end{tabular}




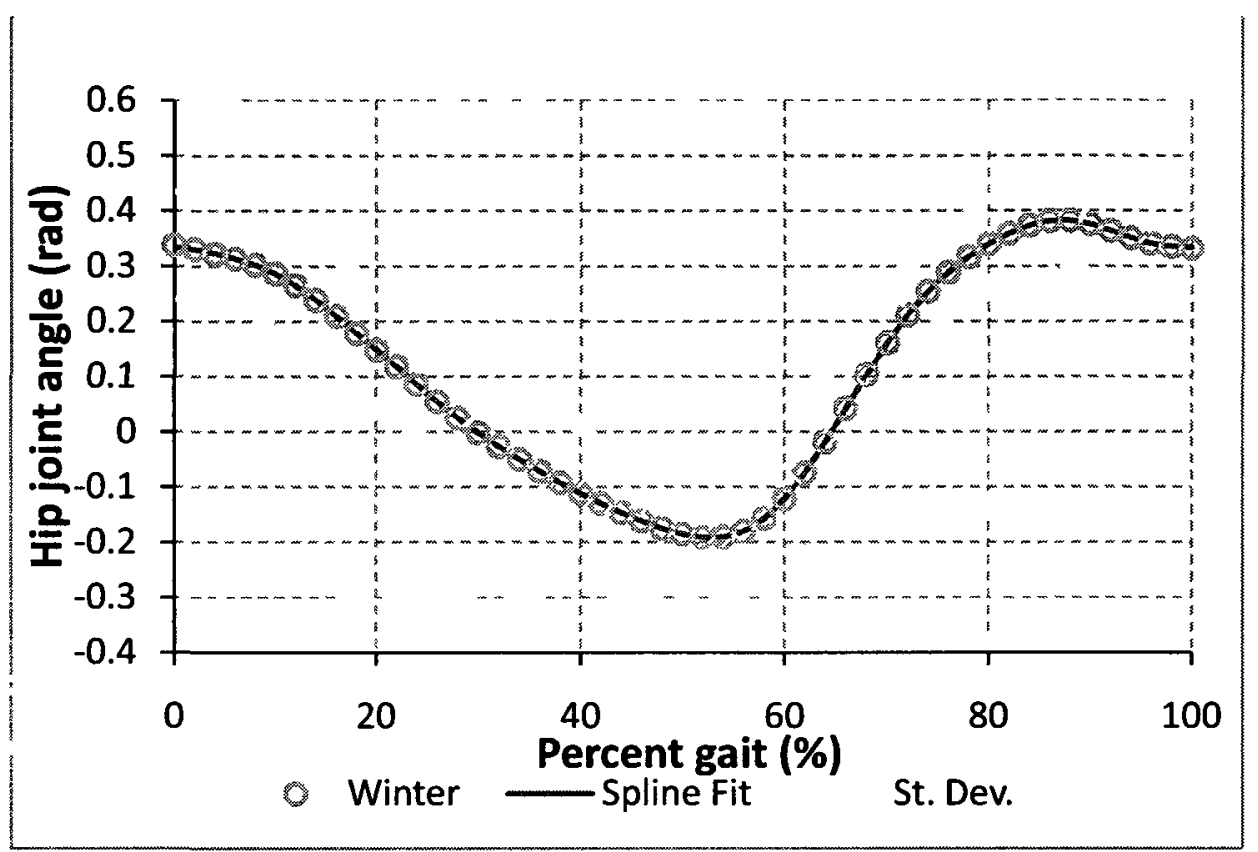

Figure 3.4 Hip Joint Angle vs Gait Cycle

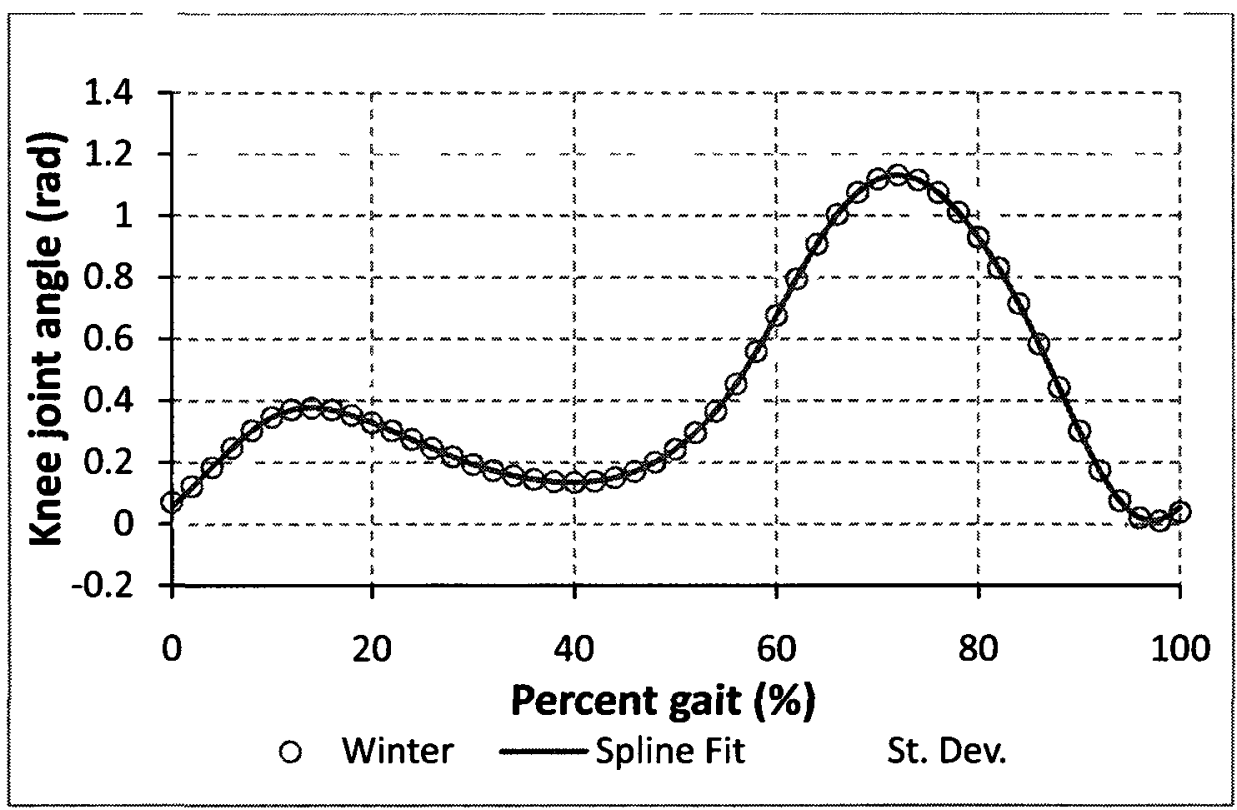

Figure 3.5 Knee Joint Angle vs Gait Cycle 


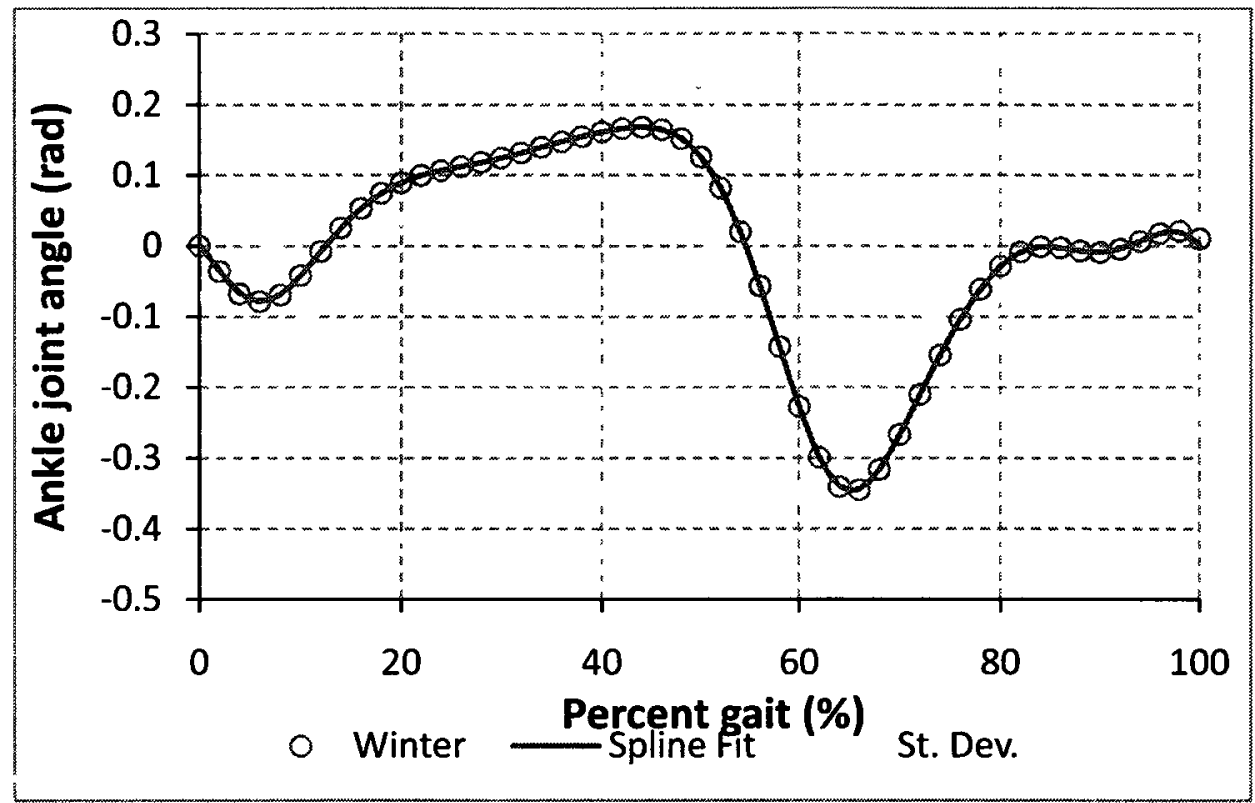

Figure 3.6 Ankle Joint Angle vs Gait Cycle

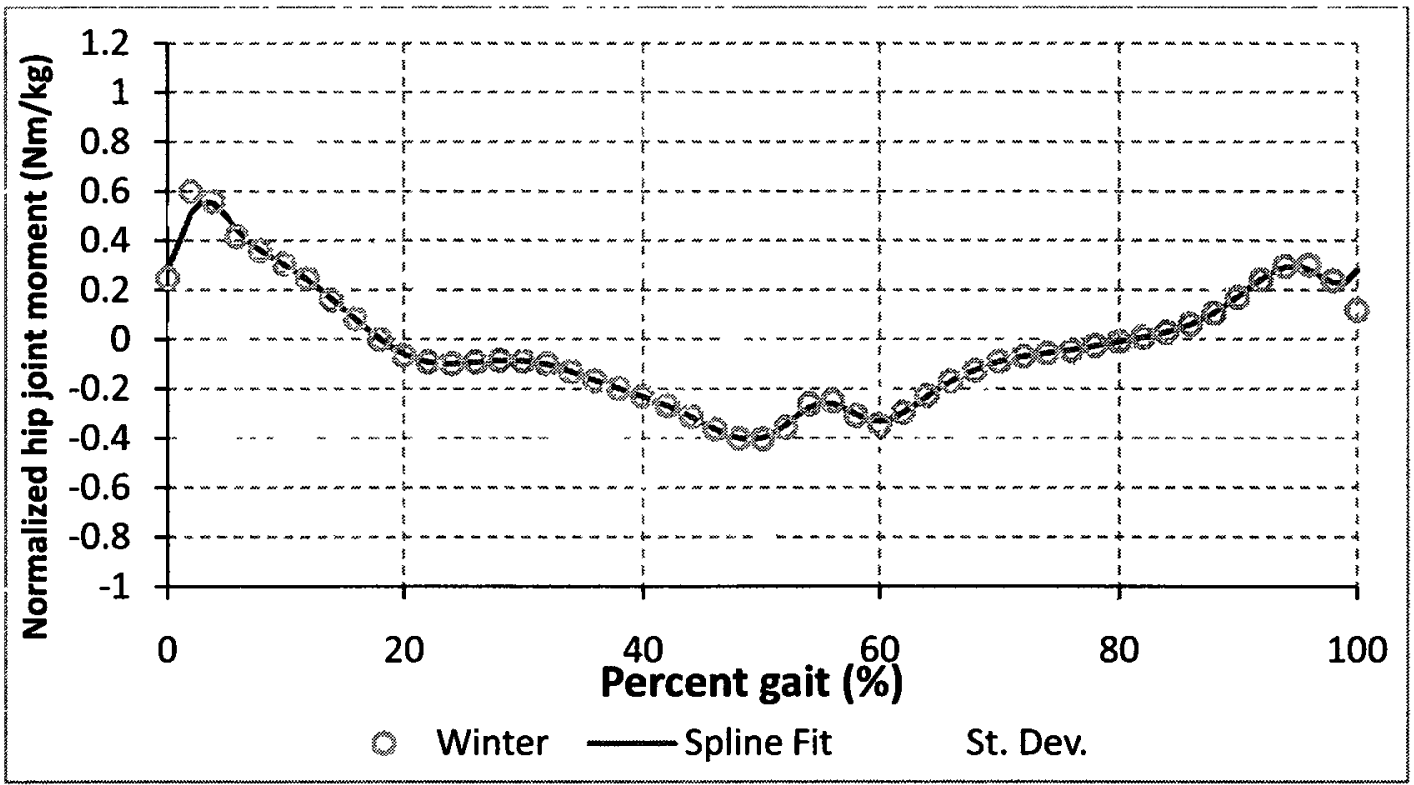

Figure 3.7: Hip Joint Moment vs. Percent of Gait Cycle 


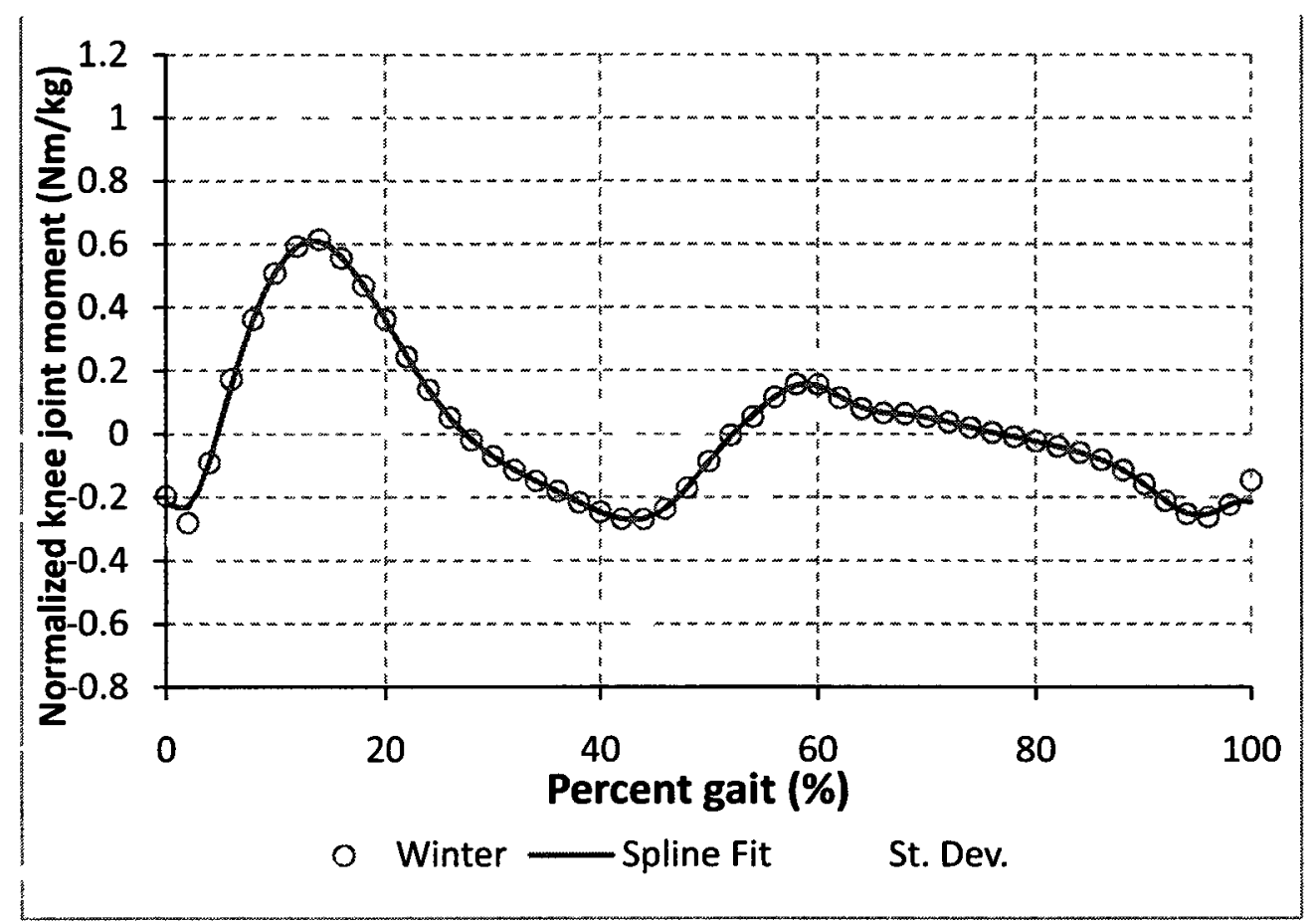

Figure 3.8: Knee Joint Moment vs. Percent of Gait Cycle

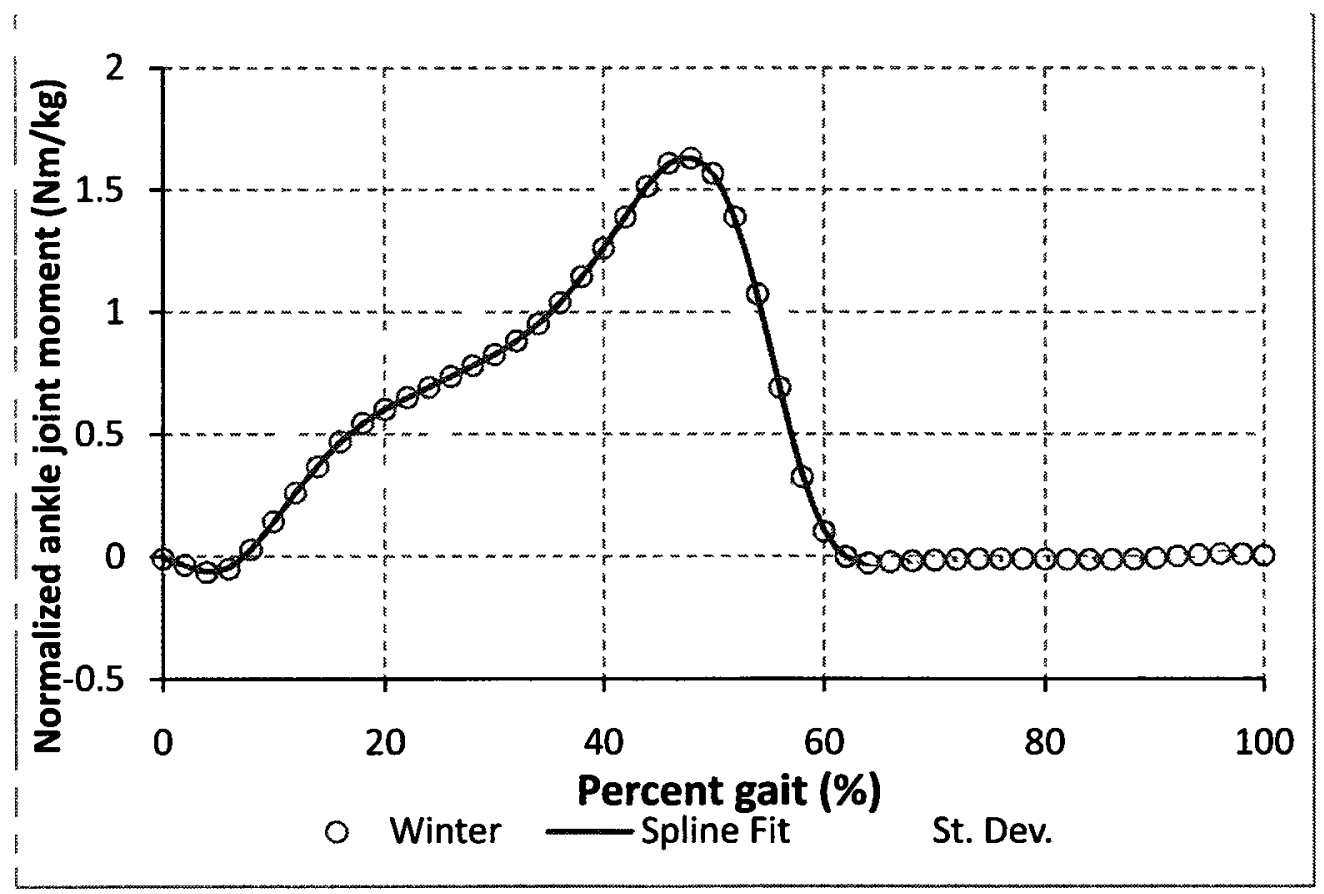

Figure 3.9: Ankle Joint Moment vs. Percent of Gait Cycle 
Four design cases were selected for loading conditions in the design process as shown in Table 3.2. These cases represent both extremes of the loading conditions with the $95^{\text {th }}$ percentile male and the $5^{\text {th }}$ percentile female loading conditions and workspaces. The 30 degree configuration represents a case partially through the rehabilitation process where the platform is rotated to allow a portion of the patient's body weight to be supported by the user while still under the assist of the manipulator, Figure 3.10. This configuration also allows the removal of a parallel body weight support system, decreasing the overall size of the platform.

Table 3.2: Rehabilitation Design Cases

\begin{tabular}{llcc}
\hline Height & Mass & $\begin{array}{c}\text { Rehabilitation } \\
\text { Angle }\end{array}$ \\
\hline Case 1 & $1.887 \mathrm{~m}$ & $88.3 \mathrm{~kg}$ & 0 degrees \\
Case 2 & $1.887 \mathrm{~m}$ & $88.3 \mathrm{~kg}$ & 30 degrees \\
Case 3 & $1.507 \mathrm{~m}$ & $74.7 \mathrm{~kg}$ & 0 degrees \\
Case 4 & $1.507 \mathrm{~m}$ & $74.7 \mathrm{~kg}$ & 30 degrees \\
\hline
\end{tabular}
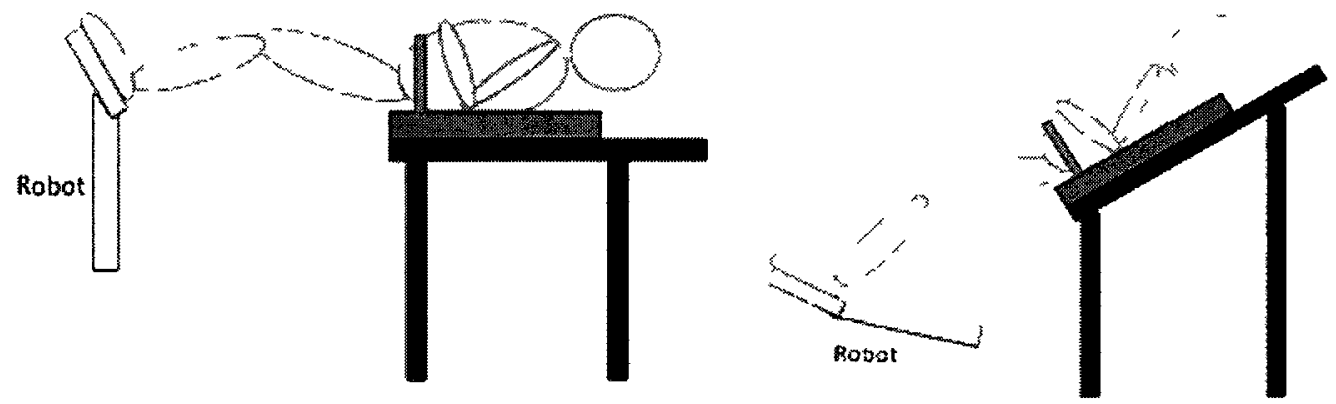

Figure 3.10 Body Positions for Rehabilitation 
The combined data was spline fit with a fifth order polynomial and differentiated to obtain the velocity and acceleration profiles of the end effector, as shown in Figure 3.11 through Figure 3.16. These figures show the male profiles. The positions were plotted with the hip as the origin.

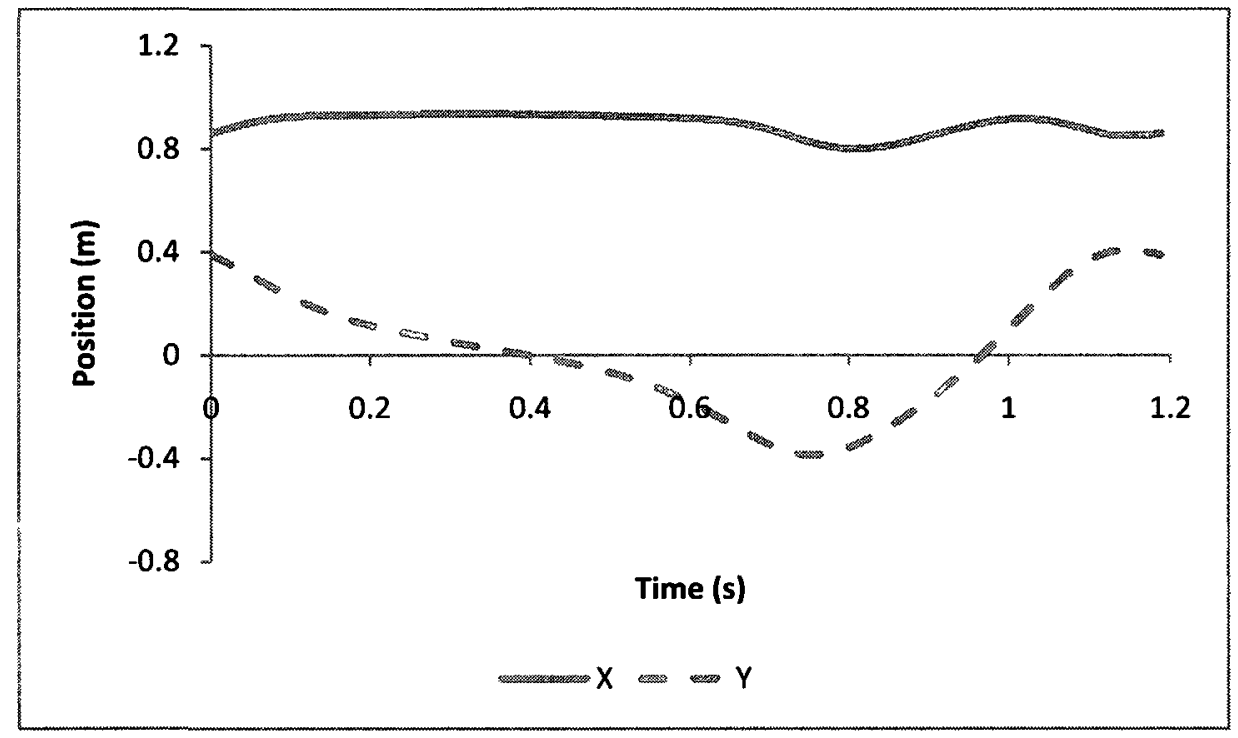

Figure 3.11 End Effector Spatial Position vs Time

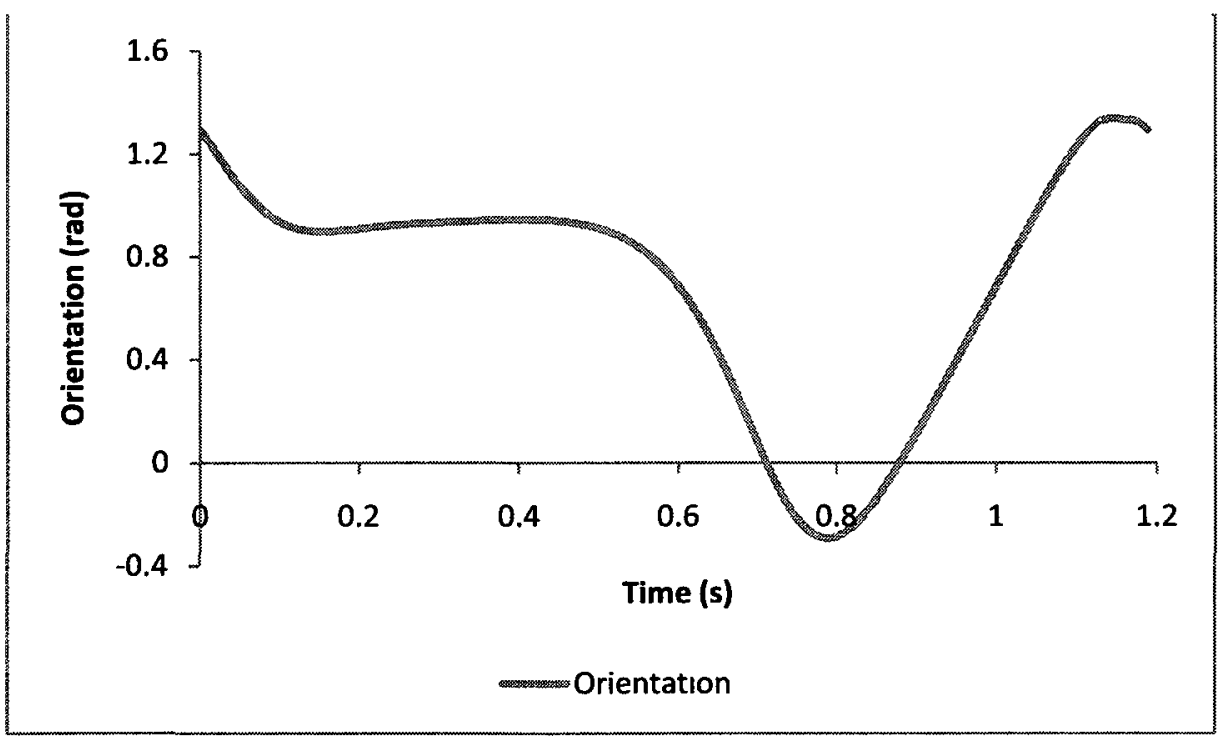

Figure 3.12 End Effector Angular Position vs Time 


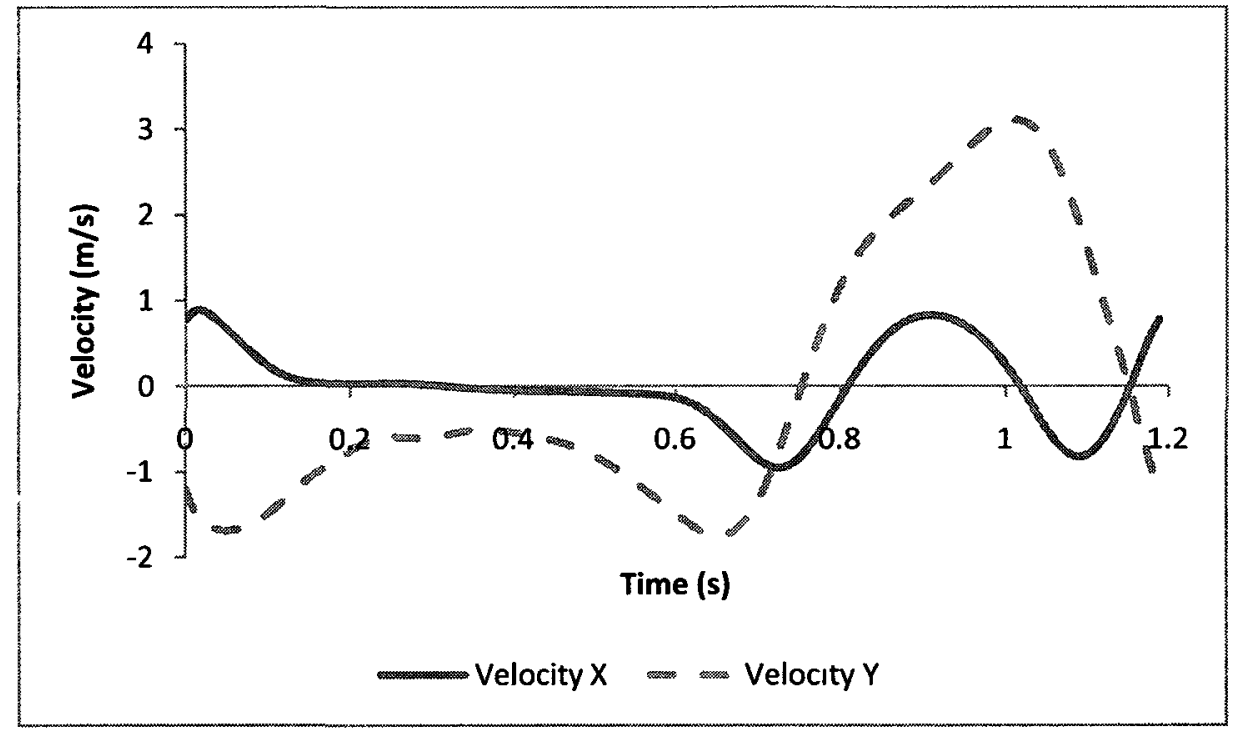

Figure 3.13 End Effector Spatial Velocity vs Time

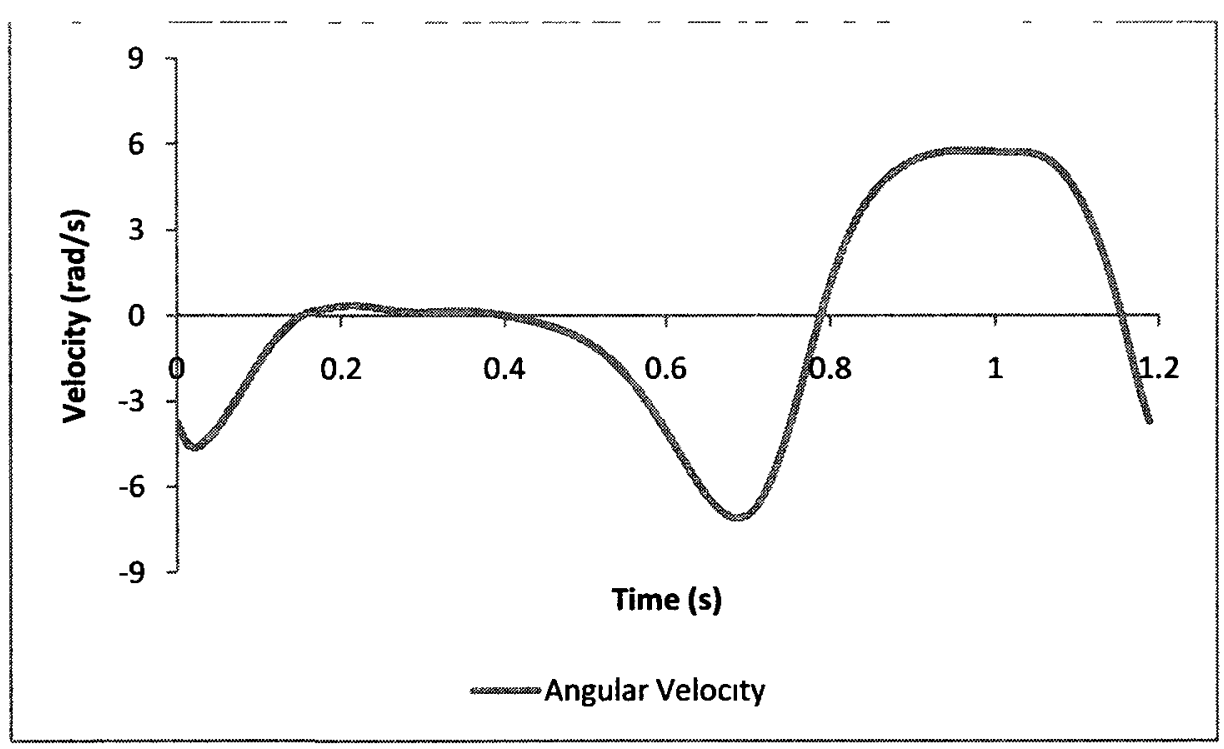

Figure 3.14 End Effector Angular Velocity vs Time 


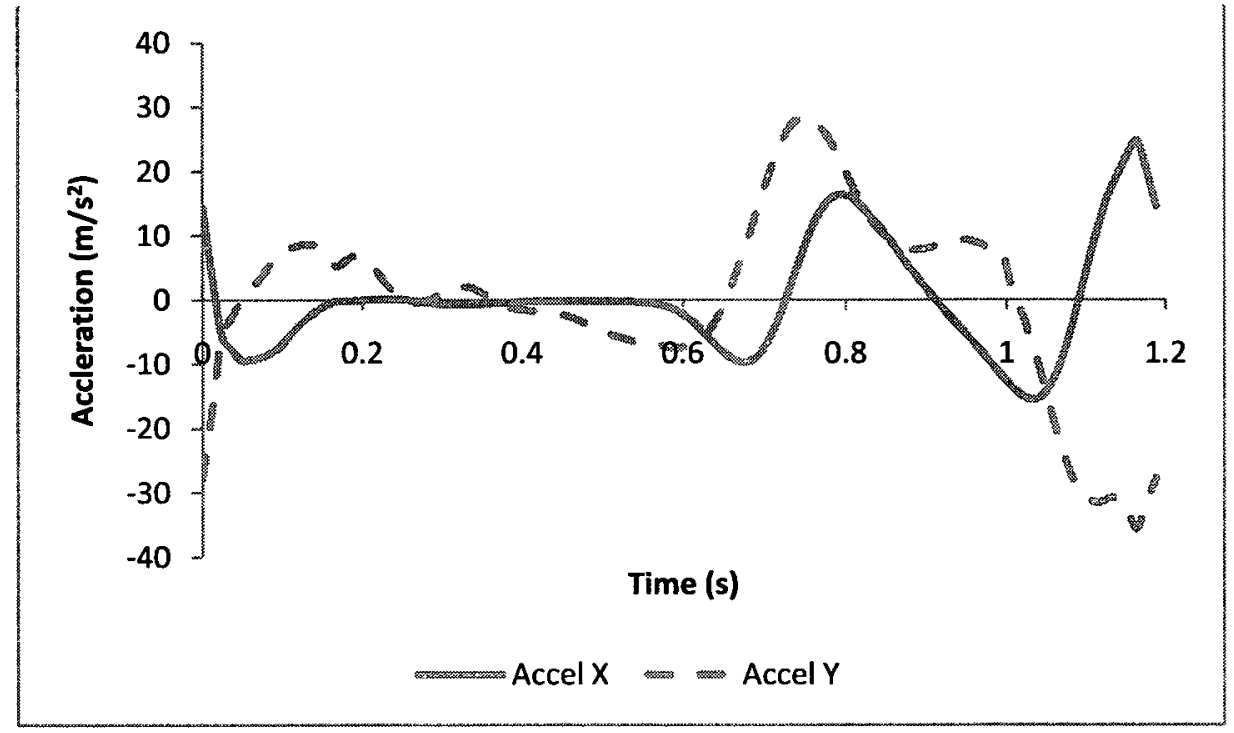

Figure 3.15 End Effector Spatial Acceleration vs Time

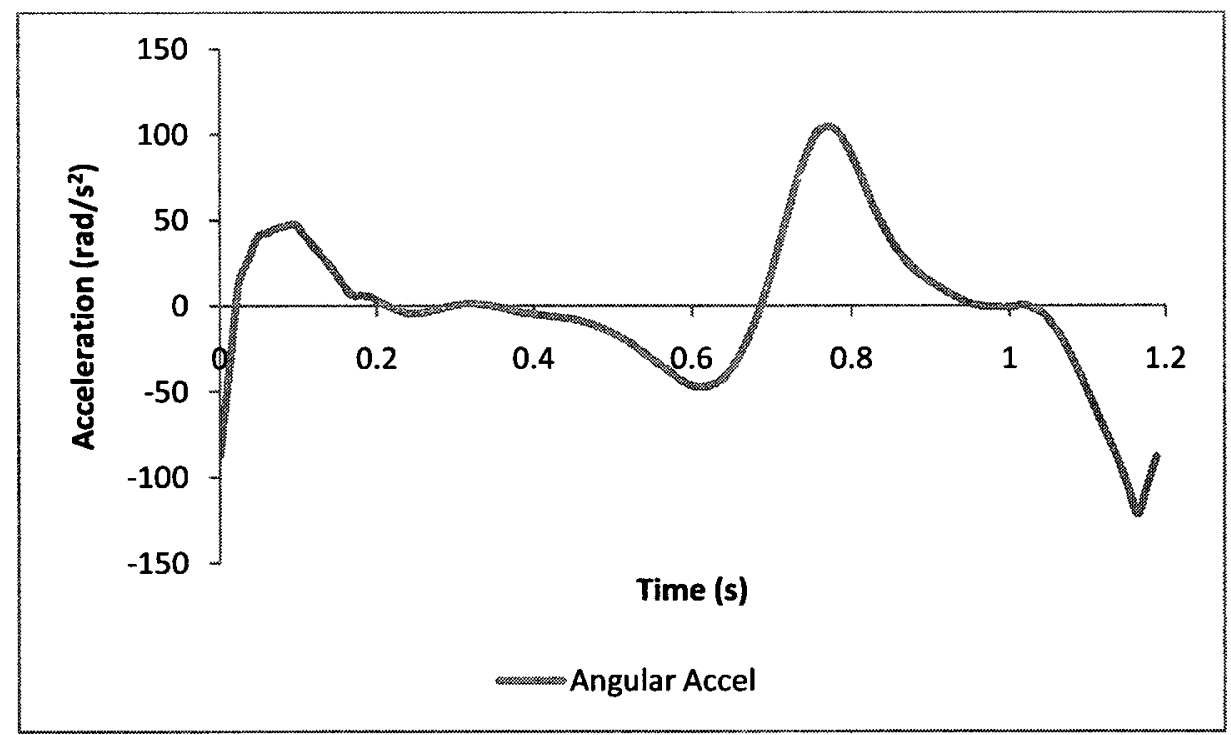

Figure 3.16: End Effector Angular Acceleration vs Time

In determining the required forces at the end effector data, Winter was again used. As the patient's leg is supported by the end effector, additional loading considerations were taken into account for the mass of the leg. Figure 3.17 and Figure 3.18 show the forces and torques, respectively, for the 0 deg male configuration. 
Though the principle design objective of the platform is for rehabilitation of gait, it is also prudent to allow the user to articulate their leg throughout the entire range of motion so that rehabilitation can cover a wider range of motion, such as ascending or descending stairs. Simply examining the joint limits of the each leg joint separately is not sufficient as the joint limits of the leg are interdependent with the angle of one joint affecting the limits of the others. Using the data presented in Riener [44] for joint limits under various body configurations and taking the maximum and minimum values for both joint angles and joint moments the workspace was defined.

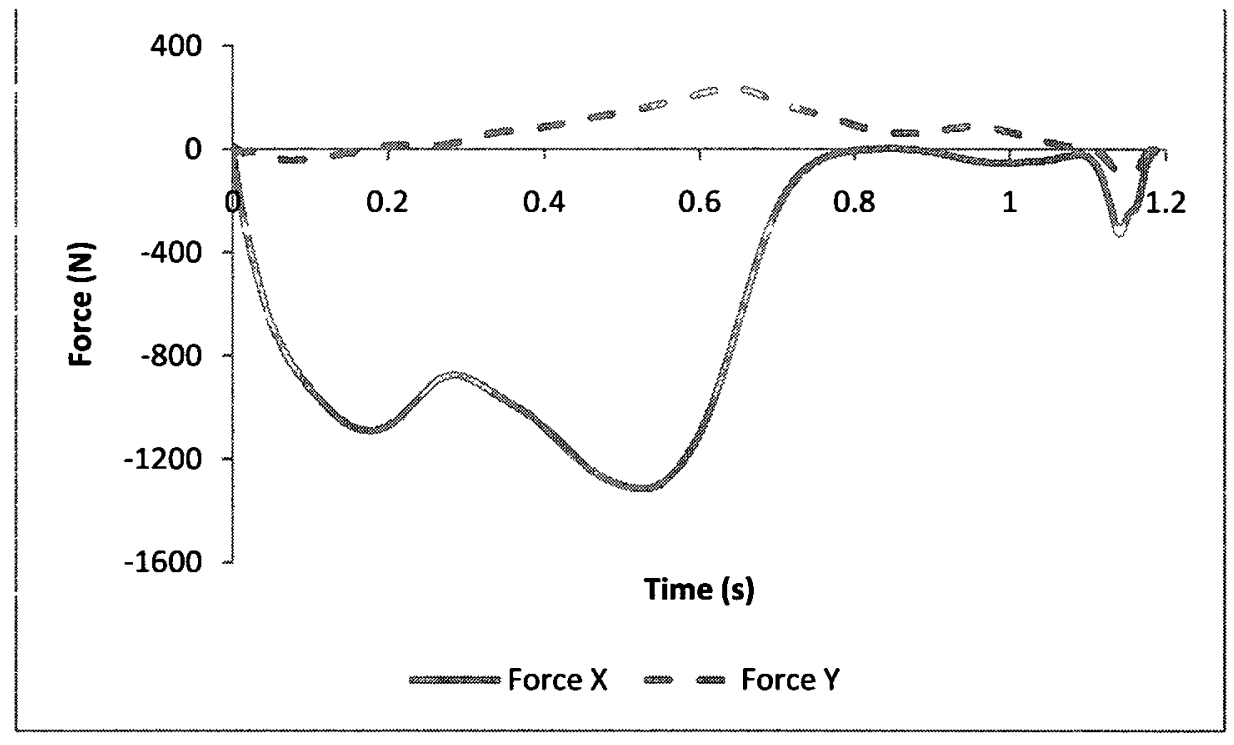

Figure 3.17 End Effector Forces vs Time 


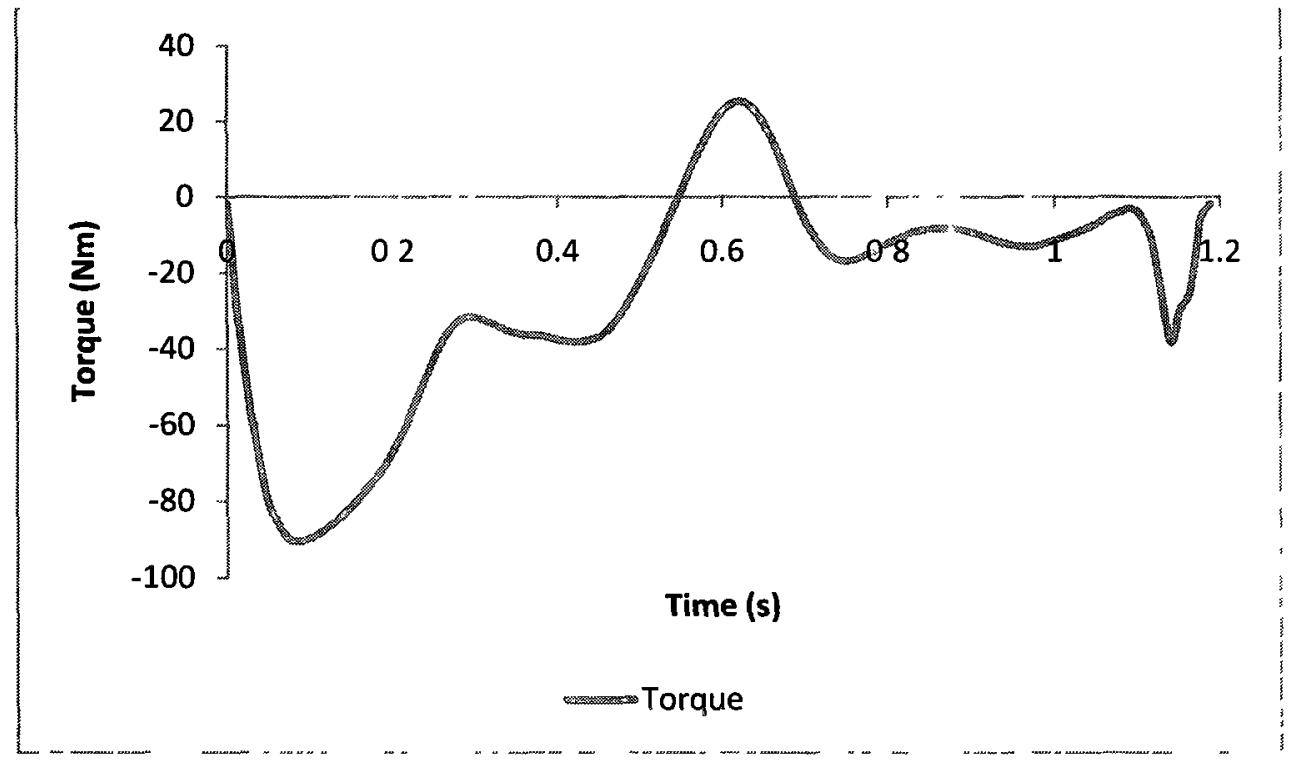

Figure 3.18 End Effector Torques vs Time

Table 3.3 Angle and Torque Limits

\begin{tabular}{l|ccc}
\hline & Joint & Flexion & Extension \\
\hline \multirow{4}{*}{ Angles } & Hip & $110 \mathrm{deg}$ & $-30 \mathrm{deg}$ \\
& Knee & $140 \mathrm{deg}$ & $0 \mathrm{deg}$ \\
& Ankle & $-20 \mathrm{deg}$ & $55 \mathrm{deg}$ \\
\hline
\end{tabular}

\subsection{Concept Platform Design Details}

One of the major drawbacks of many gait rehabilitation platforms is the size of these devices, and accordingly, their cost. As one of the end goals of this development process is to have a platform that may be used by a large range of clients, designing a system with a reduced size and cost that is easily disassembled for transport was included in all design decisions.

Classical body weight supported rehabilitation platforms as well as the robotic platforms like Lokomat and Haptic Walker also present a situation where the user is supported by a parallel harness that adds significant delays to the beginning and end of each rehabilitation session, additionally, many patients cannot support their own body 
weight. If the support system were to fail the patient may sustain additional injuries Removal of the secondary body weight support device would vastly decrease the size of the platform.

Though there was a budgetary constraint on the project, the selection of components for the manipulator was not limited by available funds. As this platform is primarily for prototyping and experimental purposes, a single leg manipulator was designed while components were purchased to allow easy scalability of the platform to include a second leg.

From the gait data and the project objective of a haptic platform with a large workspace, additional constraints were imposed in order to define the configuration of the platform prior to the detailed design. This section details these tradeoffs.

\subsubsection{Reclined Position}

To minimize the volume of the platform, a reclined user position was chosen. This places the patient with their legs extended beyond a rigid support for the upper body with the hips supported and the leg able to be articulated, as depicted in Figure 3.19 This configuration allows a significant reduction in the amount of set up time required before a therapy session would begin as there is no longer a need for the patient to be supported by a secondary body weight support harness. One drawback of this configuration is that the hips are restricted from swivelling, Future investigation to determine if this significantly affects muscle activation is required.

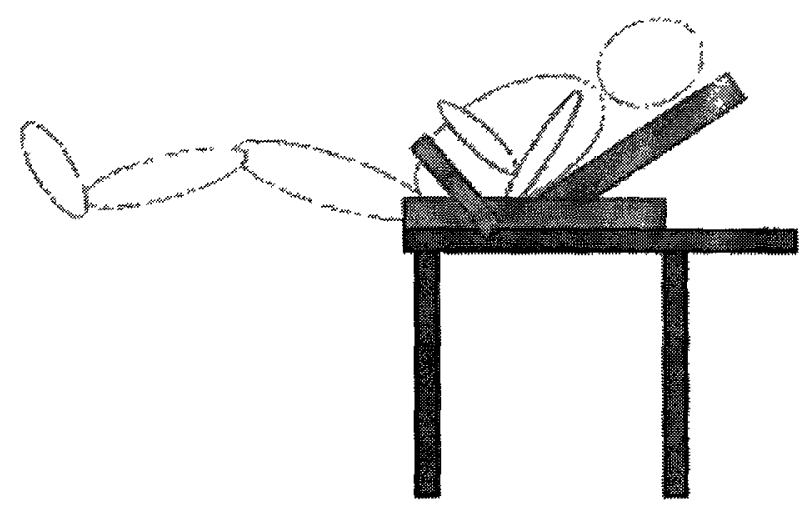

Figure 3.19 Reclined User Rehabilitation Position 


\subsubsection{Base Location}

With a broad objective of manipulating a patient's foot through a gait trajectory a number of mounting locations for the base of the robotic manipulator were considered. Mounting the manipulator in line with the patient's leg, either above or below, as in Figure 3.20 so as to decrease eccentric loading was rejected as there would be a higher incidence of collisions between the robot and the user. Mounting the manipulator below the patient's foot, Figure 3.21, similar to the Haptic Walker design [39], so as to remove the linkages from the human workspace was rejected because it would increase the overall size of the platform for the manipulator to operate within the entire workspace of the human leg.

A base location for the manipulator close to the hip of the patient, Figure 3.22, would allow the device to support the user in future design configurations as well as possibly allow anthropomorphic analysis. Adding complexity to the base location would also allow for articulation of the hips. This design also allows for a larger workspace than other options and safety features are employed to mitigate safety concerns.

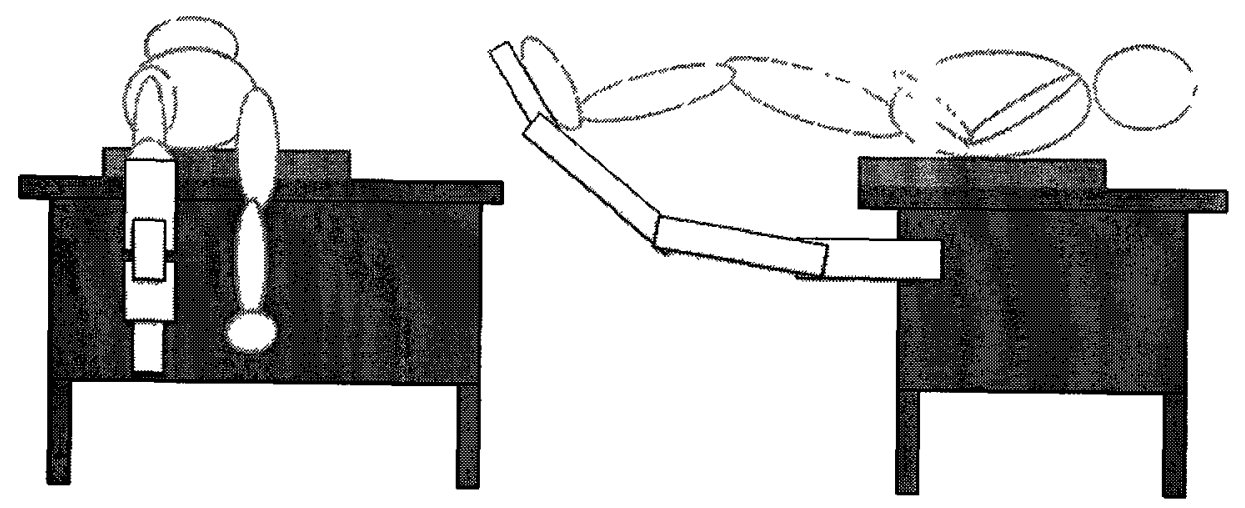

Figure 3.20: Aligned Manipulator 


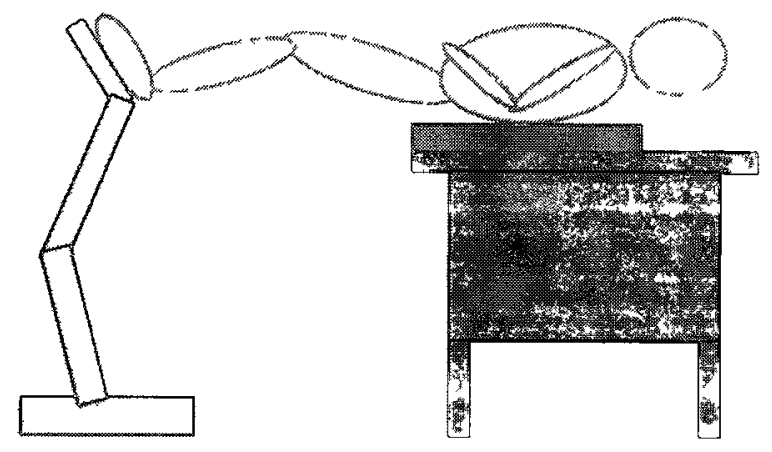

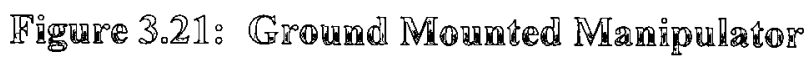

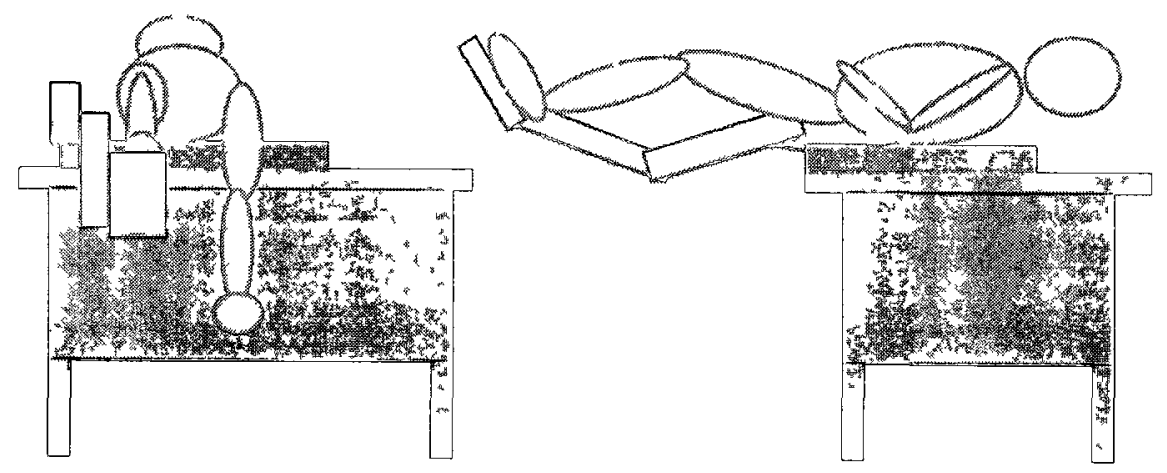

Fignure 3.22: Sidde MIoumted Mamipulator

\subsubsection{Simgle Point Atrmelnmemi at End Effector}

Designing a nom-anthropomorphic manipulator that articulates the foot rather than attaching to each section of the patient's leg allows for a single point of interaction between the user and the manipulator, Figure 3.23. This design decreases the required number of force sensors and allows for a simplified interaction model between the patient and the device. Having a single location to load the leg increases the requirements on the 
motors as the entire load to support the leg is applied through the end effector. An anthropomorphic design would require additional degrees of freedom outside of the planar motion as well as require manipulator and patient joints to be precisely aligned to allow natural motions of the leg thereby preventing injury.
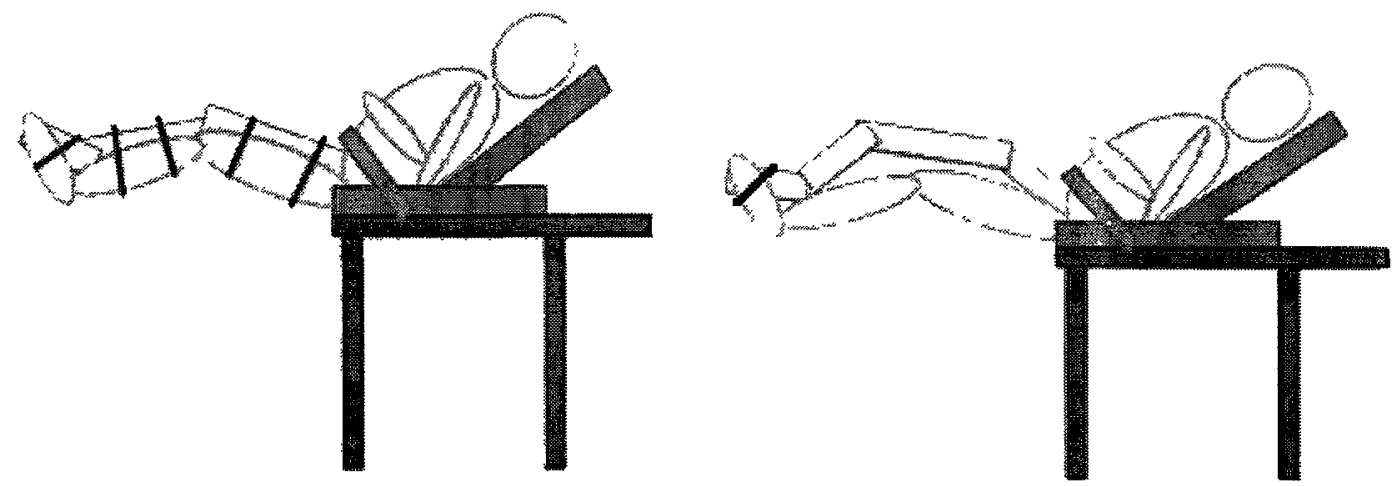

Figure 3.23: Anthropomorphic Design

\subsubsection{Planar Robot}

The human leg in total has eight degrees of freedom. The hip, a ball and socket joint has three degrees of freedom. The knee is fundamentally a hinge joint but additional flex allows for a small range of motion in a second degree of freedom. The ankle mainly functions as a two degree of freedom joint, but has movement in rotational degrees of freedom. In total, there are eight independent degrees of freedom in each leg. During a gait cycle, there is some out of plane motion, though the motion primarily happens along a plane. By actuating the foot in a planar gait, and allowing the user's leg to follow an unimpeded trajectory, the design of the manipulator is greatly simplified without constraining the leg of the patient to move in uncomfortable motions. Budgetary, resource and time constraints also limited the degrees of freedom in the device. Future plans include extending the number of degrees of freedom in the pelvis and ankle to allow a larger range of motion for the patient, including the sensation of turning, which the current system is not capable of delivery. 


\subsubsection{Revolute Joints}

In determining the layout of the platform, prismatic actuators were considered for placement at the base location in order to easily adapt to patients of various heights. One of the future modifications to ViGRR is to include a rotation of the platform so that a range of rehabilitation processes can be performed, from simple motions to full gait rehabilitation. Also, during the swing phase of the gait, while the foot is not in contact with the ground, the velocities are quite high, including a prismatic joint would require that the other joints supply a larger motion for this phase of the gait. Additionally, the forces applied to the leg during the stance phase are large and would require a significant prismatic actuator in order to sustain these loads.

\subsubsection{Parallel Support Device}

A parallel support device, such as the body weight harness for treadmill gait training, would add a secondary method of ensuring that gravity is nullified. Gravity compensation was instead included in the controller and was factored into the design of the system through maintaining the leg in a neutral position by supporting the foot. A secondary support device for the leg would have to be actively manipulated as the leg has a very large workspace. This would lead to increased cost, as well as complexity in the design and control of the system.

\subsubsection{Four Degrees of Freedom}

Adding a redundant, fourth, degree of freedom, though increasing the weight, cost and complexity of the system, allows for a more robust design by allowing the redundancy resolution scheme to minimize joint velocities, while also avoiding singular configurations, collisions and joint limits. Initially the design of the manipulator was for a three degree of freedom planar platform that could be adapted to be used anthropomorphically. The added weight, cost and complexity were deemed viable for the added robustness of the system. 


\subsection{Development of Robot Model}

\subsubsection{ViGRR Dynamics}

This section derives the equations of motion of the four revolute degrees of freedom planar manipulator. Figure 3.24 depicts the robotic naming conventions, where $\mathrm{X}_{0}, \mathrm{Y}_{0}$ is the base origin of the manipulator and $X_{n}, Y_{n}$ is the coordinate origin of link $n . l_{n}$ is the length of link $n, 1_{c n}$ is the distance from the link origin to the link center of mass, $m_{n}$ is the mass of the link and $I_{n}$ is the inertia of link $n . X_{e}, Y_{e}$ is the end effector coordinate system.

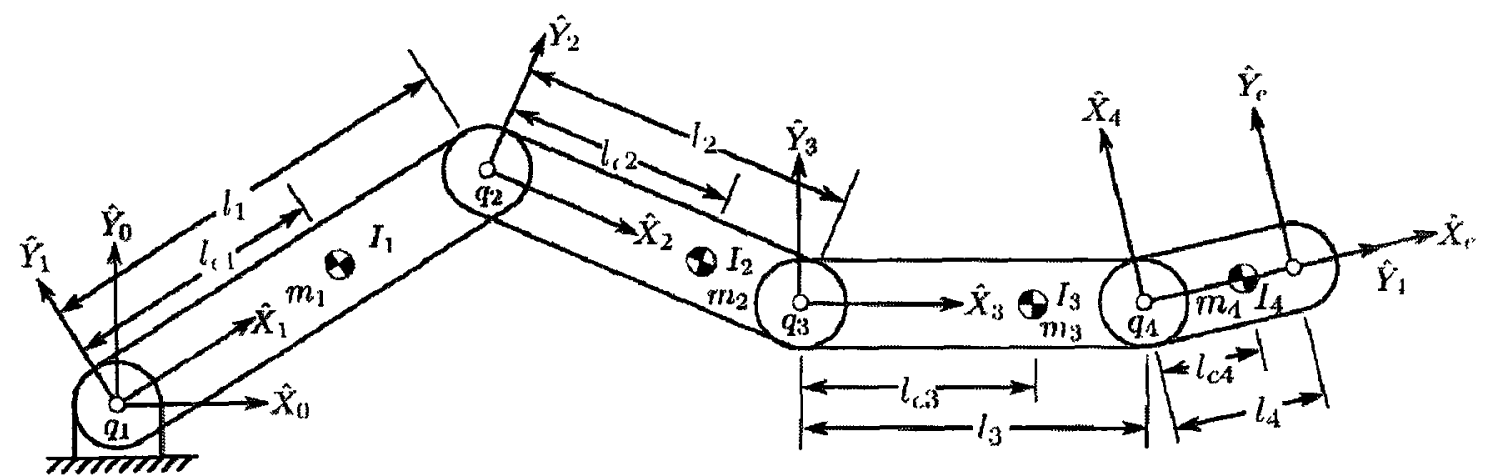

Figure 3.24: Frame, Length and Mass Definitions for the 4R ViGRR Redundant Manipulator

The dynamics for an $n=4$ link planar configuration, as derived from Euler-Lagrange formulation of robot dynamics is:

$$
\tau_{r}=M(q) \ddot{q}+C(q, \dot{q}) \dot{q}+g(q)
$$

where the subscript $\mathrm{r}$ refers to the robot, $\tau_{r}=\left[\begin{array}{llll}\tau_{1} & \tau_{2} & \tau_{3} & \tau_{4}\end{array}\right]^{T}$ are the applied torques in $\mathrm{Nm}$ and $q=\left[\begin{array}{llll}q_{1} & q_{2} & q_{3} & q_{4}\end{array}\right]^{T}$ are the relative joint angles in radians. The coefficient matrices $\mathrm{C}, \mathrm{M}$ and the vector $\mathrm{g}$ are defined below where $c_{y j}=\cos \left(q_{l}+q_{j}+\ldots+q_{m}\right)$ and similarly, $s_{y}=\sin \left(q_{t}+q_{J}+\ldots q_{m}\right)$ The full contents of the matrices are available in [45].

$$
C(q, \dot{q})=\left[\begin{array}{llll}
c_{1,1} & c_{1,2} & c_{1,3} & c_{1,4} \\
c_{2,1} & c_{2,2} & c_{2,3} & c_{2,4} \\
c_{3,1} & c_{3,2} & c_{3,3} & c_{3,4} \\
c_{4,1} & c_{4,2} & c_{4,3} & c_{4,4}
\end{array}\right]
$$




$$
\begin{gathered}
M(q)=\left[\begin{array}{llll}
m_{1,1} & m_{1,2} & m_{1,3} & m_{1,4} \\
m_{1,4} & m_{2,2} & m_{2,3} & m_{2,4} \\
m_{1,3} & m_{2,3} & m_{3,3} & m_{3,4} \\
m_{1,4} & m_{2,4} & m_{3,4} & m_{4,4}
\end{array}\right] \\
g(q)=\left[\begin{array}{l}
g_{1} \\
g_{2} \\
g_{3} \\
g_{4}
\end{array}\right]
\end{gathered}
$$

\subsubsection{ViGRR Kinematics}

\section{Forward Kinematics}

From the joint angles of the manipulator, the end effector location and orientation are defined by

$$
X_{e}=\left\{\begin{array}{l}
x_{e} \\
y_{e} \\
\psi_{e}
\end{array}\right\}=F_{r}(q)=\left\{\begin{array}{c}
l_{1} c_{1}+l_{w} c_{12}+l_{3} c_{123}+l_{3} c_{1234} \\
l_{1} s_{1}+l_{2} s_{12}+l_{3} s_{123}+l_{3} s_{1234} \\
q_{1}+q_{2}+q_{3}+q_{4}
\end{array}\right\}
$$

where $X_{e}$ is the pose of the end effector defined by the Cartesian coordinated $\left(x_{e}, y_{e}\right)$ and the orientation with respect to the global coordinate system $\psi$, the angle from $\mathrm{X}_{0}$ to $\mathrm{X}_{\mathrm{e}}$ around the $Z_{0}$ axis [45].

\section{Inverse Kinematics}

As the ViGRR manipulator is planar and has 4 degrees of freedom, there is a redundant degree of freedom. For this reason there is no single solution for the mapping of $X_{e} \in R^{3}$ to $q \in R^{4}$. There are two methods of determining the inverse kinematics presented in this section, one by constraining a joint in the manipulator and the second through the use of a redundancy resolution scheme.

By constraining the 4 th joint angle the three other joint positions determine the end effector pose. In this case the inverse kinematics are calculated by 


$$
q=\left\{\begin{array}{l}
q_{1} \\
q_{2} \\
q_{3} \\
q_{4}
\end{array}\right\}=\left\{\begin{array}{c}
a \tan 2\left(y_{3}, x_{3}\right) \pm \cos ^{-1}\left(\frac{x_{3}^{3}+y_{3}^{2}-l_{1}^{2}-l_{2}^{2}}{2 l_{1} \sqrt{x_{3}^{3}+y_{3}^{2}}}\right) \\
\pm \cos ^{-1}\left(\frac{x_{3}^{3}+y_{3}^{2}-l_{1}^{2}-l_{2}^{2}}{2 l_{1} l_{2}}\right) \\
\psi_{e}-q_{2}-q_{1}-q_{4} \\
q_{4}
\end{array}\right\}
$$

where [45]

$$
\begin{aligned}
& x_{3}=x_{e}-l_{4} \cos \left(\psi_{e}\right)-l_{3} \cos \left(\psi_{e}-q_{4}\right) \\
& y_{3}=x_{e}-l_{4} \sin \left(\psi_{e}\right)-l_{3} \sin \left(\psi_{e}-q_{4}\right)
\end{aligned}
$$

\section{Differential Kinematic Relations and Statics}

From the joint velocities, the end effector velocities can be calculated by differentiating with respect to time to obtain

$$
\dot{X}_{e}=J_{r} \dot{q}
$$

where the ViGRR Jacobian $J_{r}$ is

$$
J_{r}=\left[\begin{array}{llll}
j_{11} & j_{12} & j_{13} & j_{14} \\
j_{21} & j_{22} & j_{23} & j_{24} \\
j_{31} & j_{32} & j_{33} & j_{34}
\end{array}\right]
$$

where

$$
\begin{aligned}
& j_{11}=-l_{1} s_{1}-l_{2} s_{12}+l_{3} s_{123}-l_{4} s_{1234} \\
& j_{21}=l_{1} c_{1}+l_{2} c_{12}+l_{3} c_{123}+l_{4} c_{1234} \\
& j_{31}=1 \\
& j_{12}=-l_{2} s_{12}+l_{3} s_{123}-l_{4} s_{1234} \\
& j_{22}=l_{2} c_{12}+l_{3} c_{123}+l_{4} c_{1234} \\
& j_{32}=1 \\
& j_{34}=1
\end{aligned}
$$




$$
\begin{aligned}
& j_{13}=-l_{3} s_{123}-l_{4} c_{1234} \\
& j_{23}=l_{3} c_{123}+l_{4} c_{1234} \\
& j_{33}=1 \\
& j_{14}=-l_{4} s_{1234} \\
& j_{23}=l_{4} c_{1234} \\
& j_{34}=1
\end{aligned}
$$

To relate the forces and torques at the end effector $f_{e}=\left[f_{e x} f_{e y} \tau_{e x}\right]^{T}$ to the torques applied at the joints $\tau_{r}$, the transpose of the Jacobian is used.

$$
\tau_{h}=J_{r}^{T} f_{e}
$$

where $f_{e x}$ and $f_{e y}$ are the forces applied to the end effector in the global coordinate frame and $\tau_{\mathbf{z}}$ is the torque about the global coordinate axis $Z_{0}$ By differentiating with respect to time the relationship between the end effector and joint accelerations is derived

$$
\ddot{X}_{e}=J_{r} \dot{q}+J_{r} \ddot{q}
$$

As the robot is redundant, the Jacobian is not square and cannot be inverted. For the inverse relationship, joint accelerations with respect to the end effector accelerations, a solution called the Jacobian pseudoinverse is implemented

$$
J^{+}=J_{r}^{T}\left(J_{r} J_{r}^{T}\right)^{-1}
$$

This is used to calculate the joint velocities by

$$
\dot{q}=J^{+} \dot{X}_{e}
$$

where $\|\dot{q}\|$ is minimized by the pseudoinverse [46] [47]. The joint accelerations are calculated in the same manner where [45]

$$
\ddot{q}=J^{+}\left(\ddot{X}_{w}-\dot{J}_{r} \dot{q}\right)
$$

\subsection{Specifications and Requirements}

The ViGRR platform must manipulate the legs of a patient under haptic control at at least 100 strides a minute with a body load percentage of $60 \%$. Additionally, the platform must aid in rehabilitation of general leg motor control. From this broad specification of the task of the robot the requirements of the platform were developed and are outlined below. The 
requirements for the manipulator can be broken into two categories. 1. General requirements for a gait training manipulator and 2. application requirements that are specific to the current design. Figure 3.25 outlines the areas of general and application requirements that are discussed in this section.

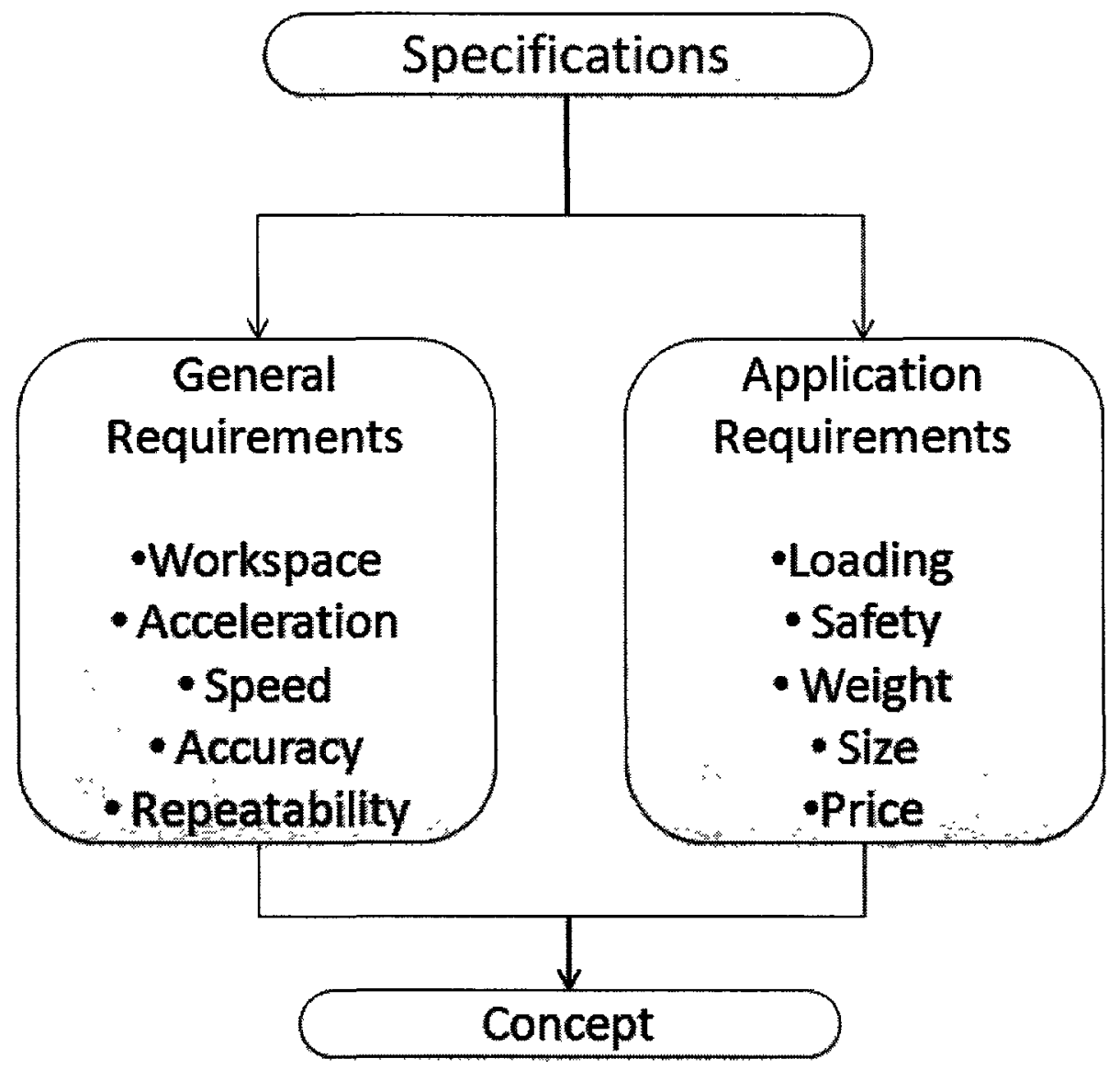

Figure 3.25: Overview of ViGRR Requirements

\subsubsection{Patient Workspace Requirement}

In order to assist in the general rehabilitation of patients with decreased control of their legs that manipulator must operate within the work envelope of a 95th percentile 
male and a 5th percentile female, not just gait workspace. From the information presented in Table 3.3 of the joint limits and Table 3.1 of the leg dimensions, and iterating the configurations with MatLAB, Figure 3.26 was developed which depicts the workspace of both of these design cases. The interior region is the workspace of the $5^{\text {th }}$ percentile female while the outer, darker, region is the $95^{\text {th }}$ percentile male.

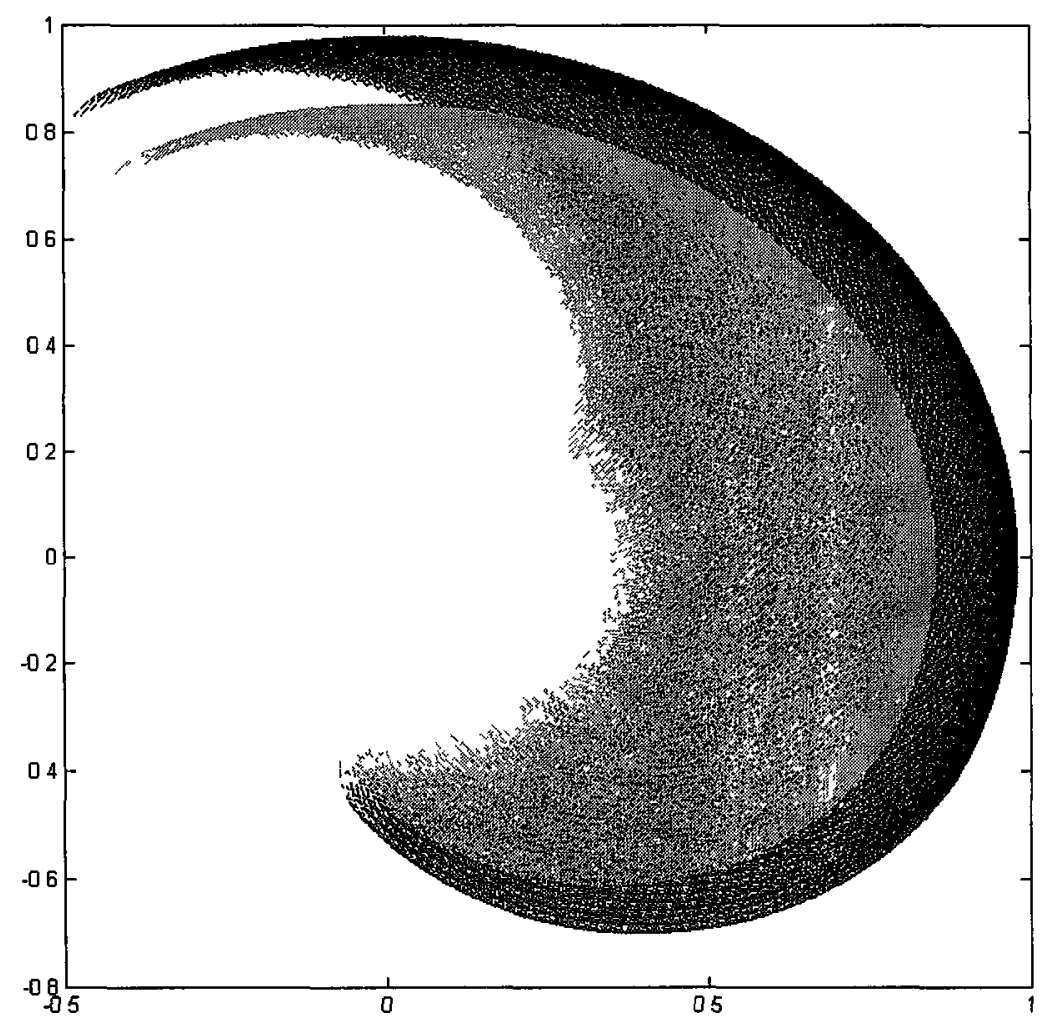

Figure 3.26: Work envelopes of a $95^{\text {th }}$ percentile man and a $5^{\text {th }}$ percentile female.

\subsubsection{Trajectory and Loading Requirements}

The device must meet the requirements of the gait trajectories parameters in order to emulate ground interaction forces and motions of walking. Interpreting the data presented in Figure 3.11 through Figure 3.18, and taking the maximum values as minimum limits for the end effector requirements the list below was formed. The optimization performed 
of the redundant manipulator to determine link dimensions also details the joint level trajectories necessary.

- End effector must have a maximum velocity of at least $3 \mathrm{~m} / \mathrm{s}$

- End effector must have a maximum angular velocity of at least $7 \mathrm{rad} / \mathrm{s}$

- End effector must have a maximum acceleration of at least $40 \mathrm{~m} / \mathrm{s}^{2}$

- End effector must have a maximum angular acceleration of at least $120 \mathrm{rad} / \mathrm{s}^{2}$

- Manipulator structure must have a load capacity of at least $1500 \mathrm{~N}$

\subsubsection{Stiffness Requirement}

A haptic device requires a highly rigid manipulator to realistically impart the virtual environment to the user. This requires that there be minimal backlash in the actuators and that the structure be highly rigid. The gait cycle requires loads over $1000 \mathrm{~N}$ be accurately imparted to the user, as such a maximum deflection of the end effector was set at $20 \mathrm{~mm}$ under static loading of the worst case load dynamic loads.

\subsubsection{Safety Requirements}

ViGRR operates parallel to the human leg and in close proximity, in order to decrease the likelihood of injury due to collisions with the user. The robot should operate entirely outside of the workspace of the patient.

As there is a risk of hyper-extension, as well as flexion, of the user's joints the controller must include sufficient data on the user in order to prevent incompatible configurations. Additionally, limits preventing motions that are outside of the velocity or torque capacities of a human leg must also be put in place.

Device must include multiple levels of safety to prevent the user from additional injury during use due to hyperextension of joints, collisions with the robot or any other unforeseen circumstance.

Lastly, the end effector must be designed in such a way that the foot is disengaged from the manipulator under a hard press against the device for cases where other safety features do not enable before injury would be possible. 


\subsubsection{Requirements Summary}

The manipulator as a haptic gait and leg rehabilitation platform must meet the following requirements:

- Operate within the workspace of the human leg,

- Meet the trajectory requirements derived in Section 3.1,

- Maintain a stiff haptic interface under $100 \%$ gait loading with a maximum deflection of $20 \mathrm{~mm}$,

- Parallel designed redundant safety mechanisms to diminish the likelihood of injury.

\subsection{Optimal Design and Component Selection Process}

Following the design flow in Figure 3.27, the design of the ViGRR platform was iterated in order to determine the lengths of the links, and the drive system that would be used to articulate the platform.

Using the required end effector trajectories and loading, and initial approximates for the weight properties of the links and the end effector, the design was iterated to determine viable link lengths and associated joint torques and velocities. These were used to select initial drive components and the mass properties were fed back into the link sizing. New optimized lengths and joint torques and velocities were output. The optimization determined appropriate link lengths for the velocity profiles of the motors while minimizing torques, and optimizing the singularity avoidance and joint limit avoidance coefficients for the redundancy resolution scheme.

\subsubsection{Link Sizing}

In this iterative design process, each of the link lengths were optimized, a constraint was imposed on all link lengths, the limits of the optimization were set between 0.1 meters and 0.7 meters for links 1 through 3 , while the fourth link was bounded between 0.1 and 0.4. Also included was an offset angle between the link and the fixed orientation of the end effector, bounded between $-10^{\circ}$ and $90^{\circ}$. The offset angle is the angle between 
the interface plate of the end effector and the final link, Figure 3.28. Additionally in the optimization of these parameters the joint limit avoidance and singularity avoidance coefficients were optimized for the redundancy resolution scheme presented in [45].

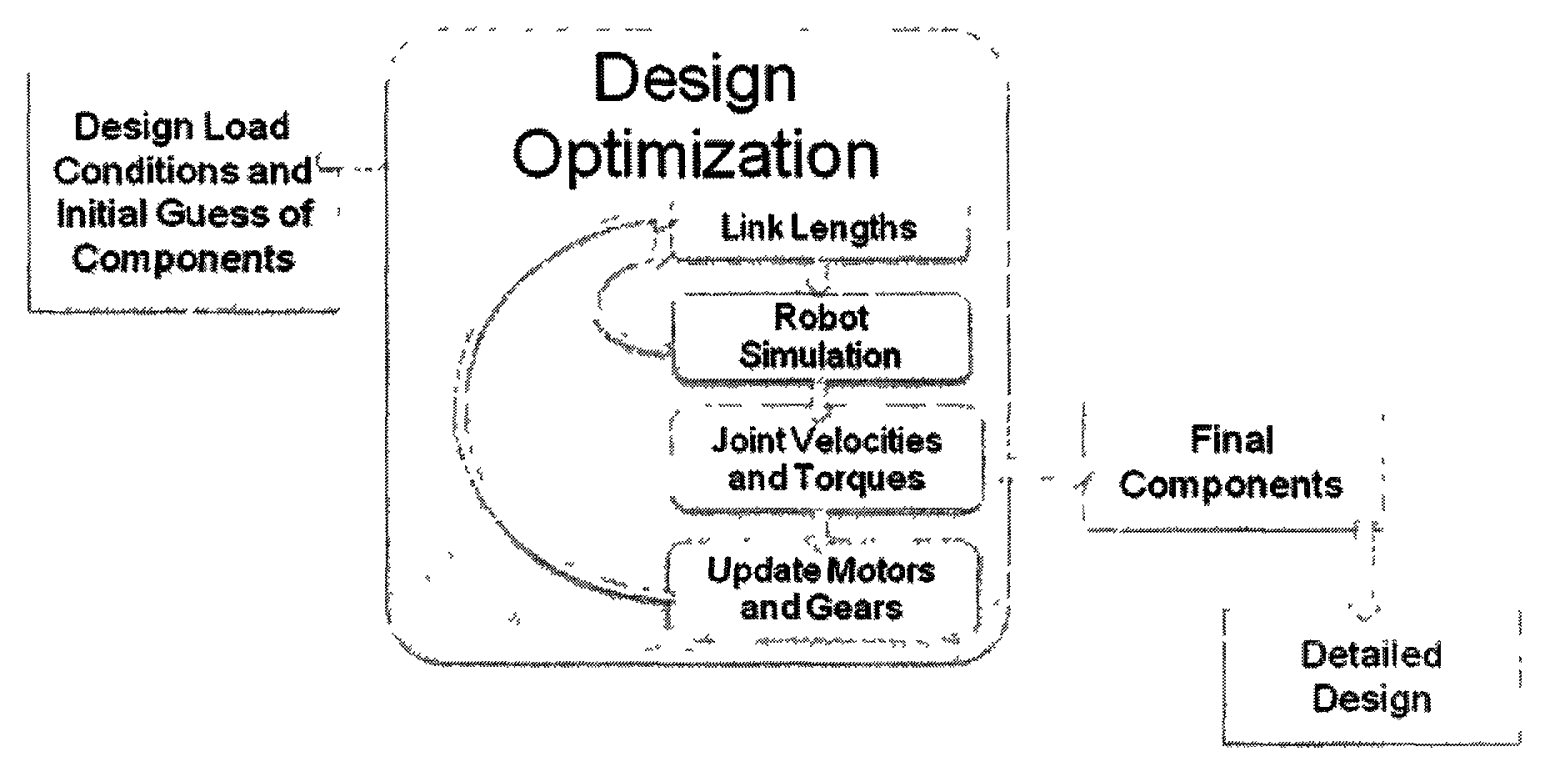

Figure 3.27: Component Selection Iterative Optimization

Using the end effector loading conditions and the dimensions of the user's leg, estimating the link masses per unit length and importing the motor and gearing data for the selected components the optimization was performed using the inverse kinematics and inverse dynamics of the manipulator. The optimized link parameters and associated torque and angular velocity curves of the motors were plotted and compared to the limits of the components. From these results, new hardware components were selected and the optimization re-run. Table 3.4 presents the results of the optimization for the parameters optimized while the next section discusses the components selected. 


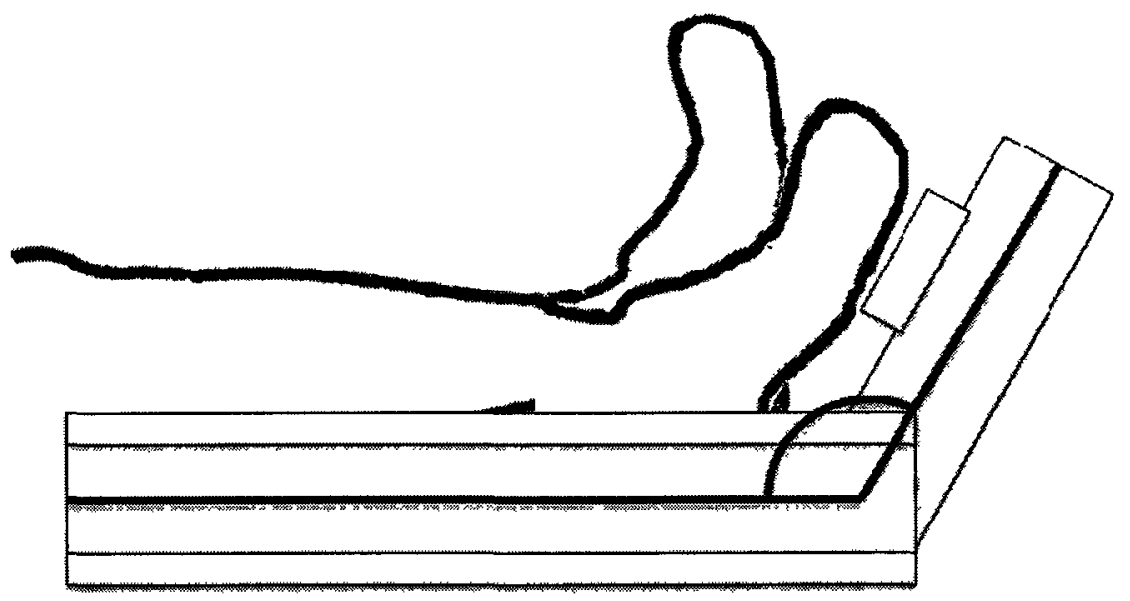

Figure 3.28: End Effector Offset Angle

Table 3.4: Optimized Design Values

\begin{tabular}{ccccccc}
\hline $\mathrm{l}_{1}(\mathrm{~m})$ & $\mathrm{l}_{2}(\mathrm{~m})$ & $\mathrm{l}_{3}(\mathrm{~m})$ & $\mathrm{l}_{4}(\mathrm{~m})$ & $\alpha(\mathrm{deg})$ & $\mathrm{k}_{\text {sing }}$ & $\mathrm{k}_{\mathrm{llm}}$ \\
\hline 0.2005 & 0.3441 & 0.2749 & 0.1515 & 33.47 & 0.0962 & 0.0165 \\
\hline
\end{tabular}

\subsubsection{Motor and Gear Selection}

As the sizing of the components is an iterative process a clear set of joint level torques and velocities could not be determined prior to completing the iterative process. Only Harmonic Drive gearings were considered due to their low backlash. Planetary gearboxes were found to have $4 \mathrm{arc} / \mathrm{min}$ of precision while the Harmonic Drive components had 0.5 arc/min. A library was compiled of brushless DC motors from Danaher, Cleveland Motion Controls, Applied Motion and Berger Lahr for our application and the iterative optimization was performed.

The converged components are listed in Table 3.5. The Danaher AKM 42J and 43K motors require $240 \mathrm{VAC}$ while the motors for joints three and four, $\mathrm{AKM} 22 \mathrm{E}$ use the standard 120 VAC. Table 3.6 outlines the parameters of the Danaher components while Table 3.7 details the specifications of the Harmonic Drive components. The combined 
parameters are listed in Table 3.8. Additional information on the actuation components can be found in [48], [49] and [50] for the Harmonic Drives, motors and drives respectively.

Table 3.5: Configured Drive Components

\begin{tabular}{c|ccc}
\hline Joint & Motor & Harmonic Drive & S200 Drive \\
\hline 1 & AKM42J-ACCNC-00 & CSF-32-120 & S21260-TVS \\
2 & AKM43K-ACCNC-00 & CSF-32-120 & S21260-VTS \\
3 & AKM22E-ACCNC-00 & CSG-25-160 & S20360-VTS \\
4 & AKM22E-ACCNC-00 & CSG-20-100 & S20360-VTS \\
\hline
\end{tabular}

Table 3.6: Motor and Drive Properties

\begin{tabular}{r|cccc}
\hline & Units & AKM22E & AKM42 & AKM43 \\
\hline Peak Torque & $\mathrm{Nm}$ & 2.42 & 11.6 & 16.3 \\
Max Speed & $\mathrm{rpm}$ & 5410 & 6000 & 6000 \\
Peak Current & $\mathrm{A}_{\mathrm{rms}}$ & 9 & 33.7 & 38.3 \\
Continuous Rated & $\mathrm{Nm}$ & 0.81 & 2.38 & 2.62 \\
Rated Speed & $\mathrm{rpm}$ & 3500 & 6000 & 6000 \\
Rated Power & $\mathrm{kW}$ & 0.30 & 1.5 & 1.65 \\
Torque Constant & $\mathrm{Nm} / \mathrm{A}_{\mathrm{rms}}$ & 0.32 & 0.43 & 0.52 \\
Back EMF Constant & $\mathrm{V} / \mathrm{k}_{\mathrm{rpm}}$ & 20.4 & 27.5 & 33.2 \\
Resistance & & 5.09 & 0.80 & 0.70 \\
Inductance & $\mathrm{mH}$ & 9.7 & 3.1 & 2.9 \\
Inertia & $\mathrm{kgm}$ & $0.161 \times 10^{-4}$ & $1.5 \times 10^{-4}$ & $2.1 \times 10^{-4}$ \\
Weight & $\mathrm{kg}$ & 1.1 & 3.39 & 4.35 \\
Static Friction & $\mathrm{Nm}$ & 0.005 & 0.026 & 0.038 \\
Viscous Damping & $\mathrm{Nm} / \mathrm{k}_{\mathrm{rpm}}$ & 0.0055 & 0.013 & 0.017 \\
\hline
\end{tabular}


Table 3.7: Harmonic Drive Properties

\begin{tabular}{l|llll}
\hline & Units & CSF-32-120 & CSG-25-160 & CSG-20-100 \\
\hline \hline Rated Torque at 2000 & $\mathrm{Nm}$ & 137 & 87 & 52 \\
Repeated Peak Torque & $\mathrm{Nm}$ & 353 & 229 & 107 \\
Average Torque & $\mathrm{Nm}$ & 216 & 140 & 64 \\
Momentary Peak Torque & $\mathrm{Nm}$ & 686 & 408 & 191 \\
Maximum Input Speed & $\mathrm{rpm}$ & 4800 & 5600 & 6500 \\
Average Input Speed & $\mathrm{rpm}$ & 3500 & 3500 & 3500 \\
Moment of Inertia & $\mathrm{kgm}^{2}$ & $1.69 \times 10^{-4}$ & $0.413 \times 10^{-4}$ & $0.193 \times 10^{-4}$ \\
\hline
\end{tabular}

Table 3.8: Joint Properties

\begin{tabular}{l|lllll}
\hline & Units & Joint 1 & Joint 2 & Joint 3 & Joint 4 \\
\hline Max Torque & $\mathrm{Nm}$ & 892 & 892 & 309 & 191 \\
Nominal Torque & $\mathrm{Nm}$ & 254 & 281 & 90.7 & 56.7 \\
Max Velocity & $\mathrm{rad} / \mathrm{s}$ & 5.236 & 5.236 & 4.909 & 8.378 \\
Nominal Velocity & $\mathrm{rad} / \mathrm{s}$ & 2.618 & 2.182 & 2.291 & 3.665 \\
$\begin{array}{l}\text { Torque Constant } \\
\text { Command Gain }\end{array}$ & $\mathrm{Nm} /$ Amp & 0.41992 & 0.51172 & 0.31641 & 0.31641 \\
$\begin{array}{l}\text { Encoder pulses per } \\
\text { revolution }\end{array}$ & Amp/Volt & 3.00014 & 3.00014 & 0.90004 & 0.74965 \\
\hline
\end{tabular}

Figure 3.29 through Figure 3.36 show the output of the motor and joint optimization. The plots show the estimated joint torques and velocities over a gait cycle. The dotted lines on the plots represent the maximum torque and velocities of the drive units being analysed while the dashed is the continuous maximum. The dash dot line represents the root mean square of the torques and velocities. A perfect sizing from the optimization would have the RMS value exactly the same as the continuous torque and velocity values. 


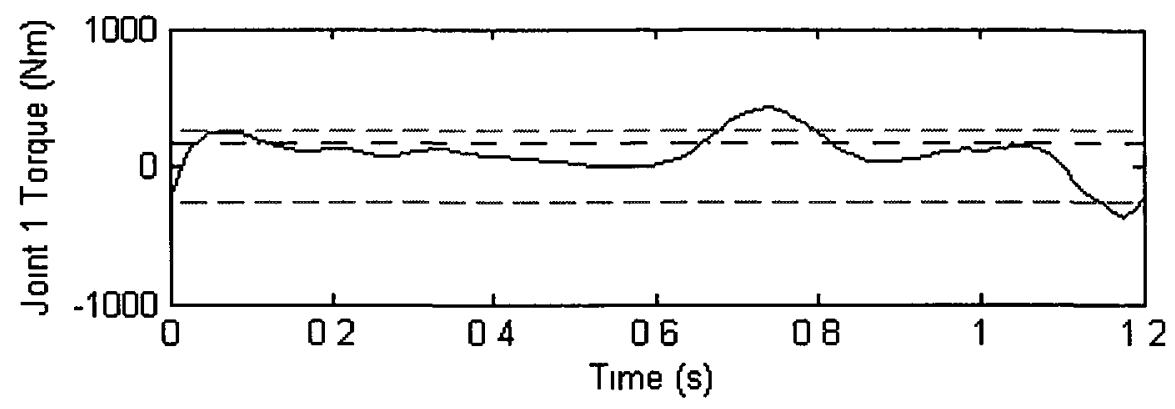

Figure 3.29: Motor Sizing - Joint 1 Torque

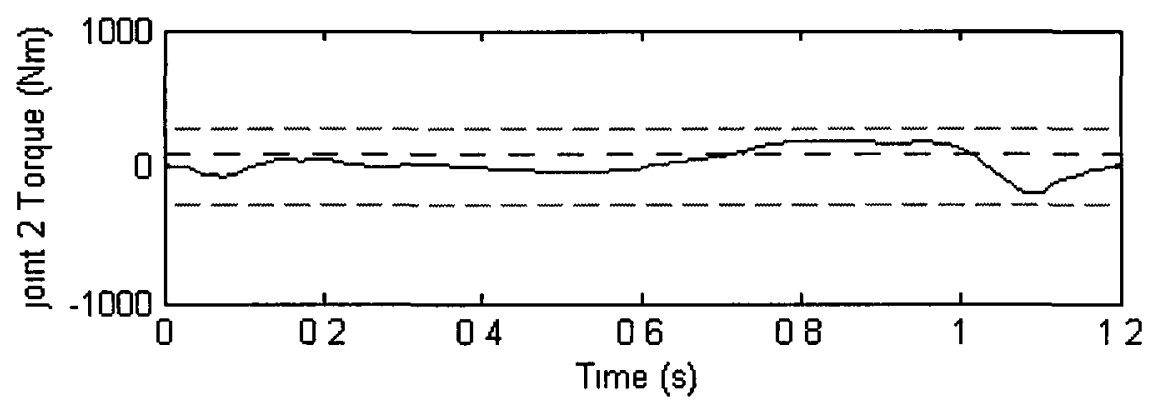

Figure 3.30: Motor Sizing - Joint 2 Torque

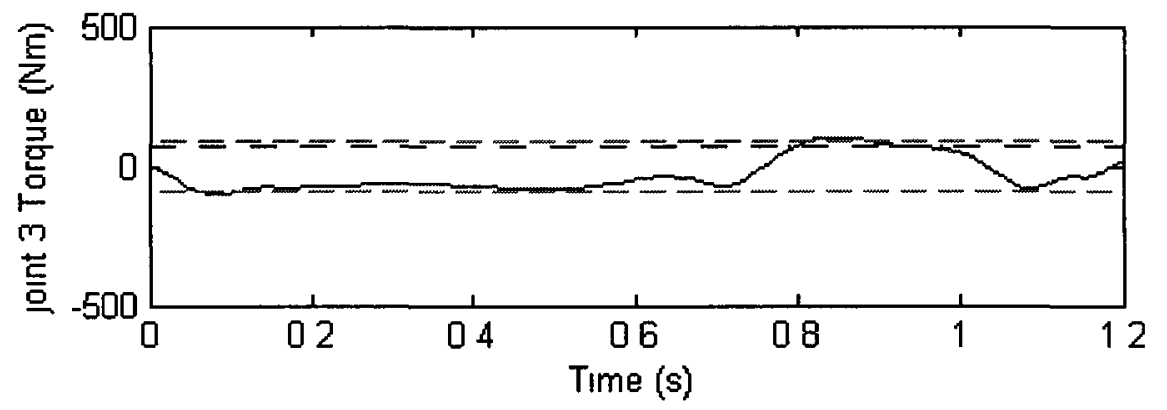

Figure 3.31: Motor Sizing - Joint 3 Torque 


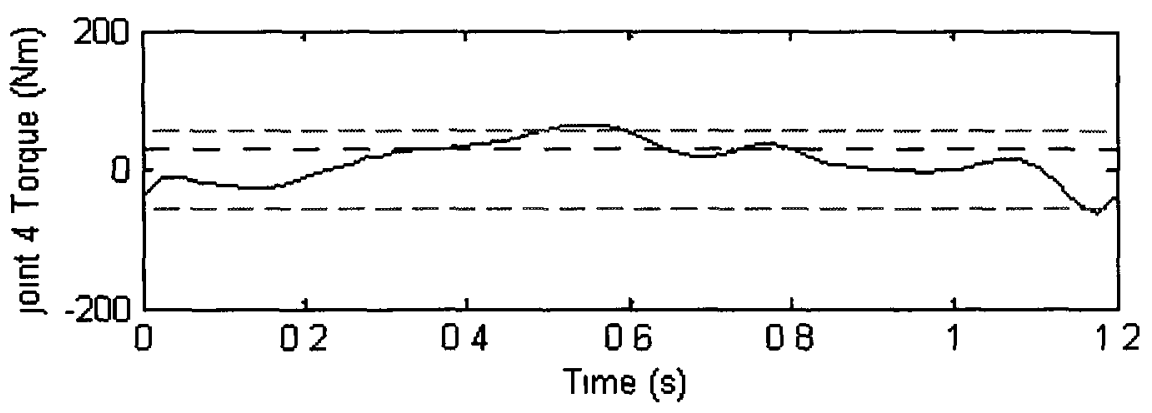

Figure 3.32: Motor Sizing - Joint 4 Torque

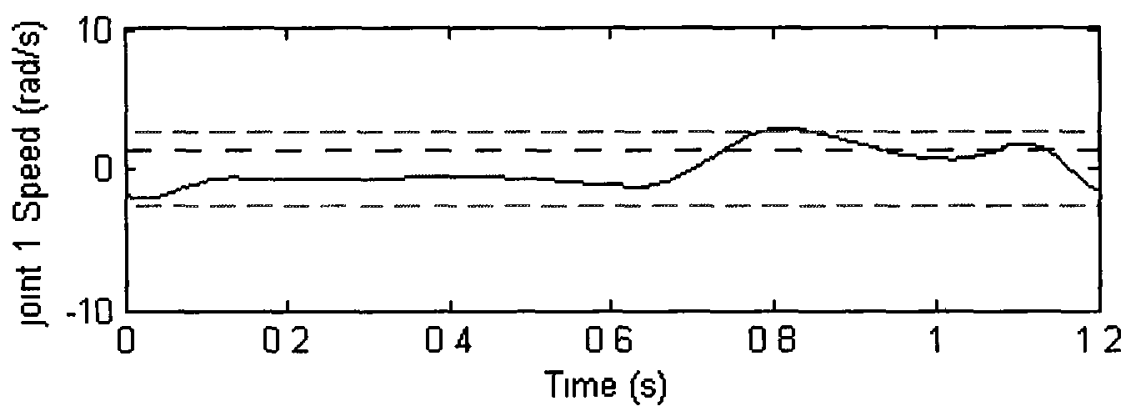

Figure 3.33: Motor Sizing - Joint 1 Speed

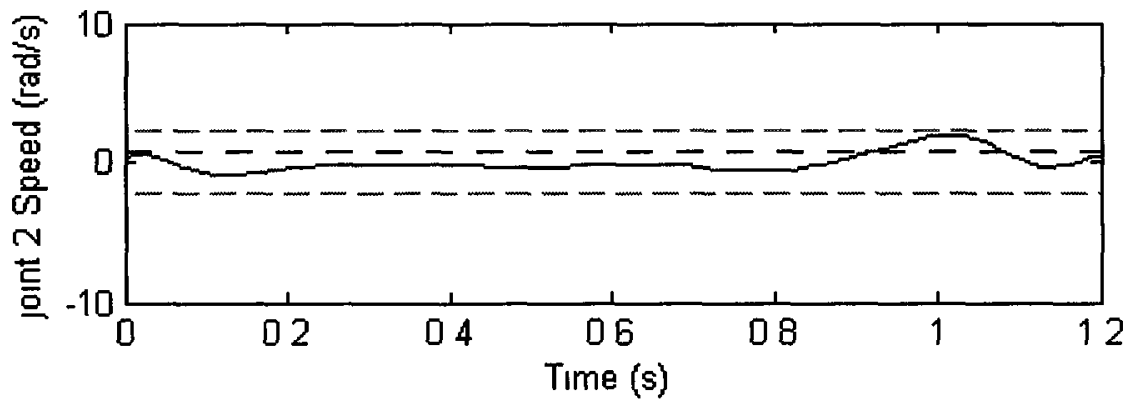

Figure 3.34: Motor Sizing - Joint 2 Speed 


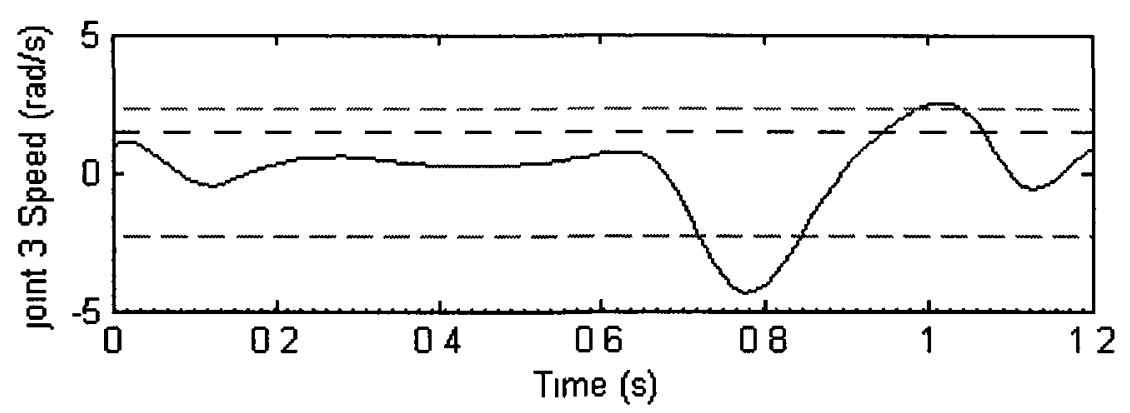

Figure 3.35: Motor Sizing - Joint 3 Speed

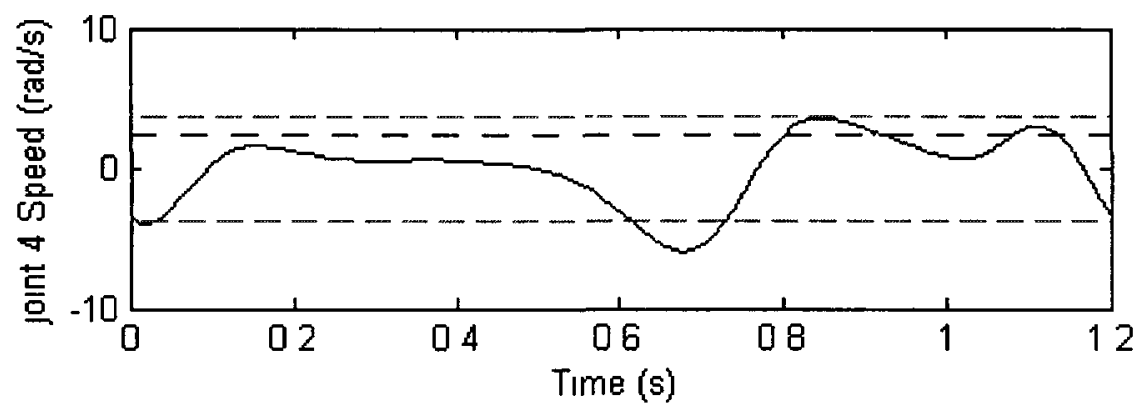

Figure 3.36: Motor Sizing - Joint 4 Speed

The assembled actuator components for joint 4 are shown in Figure 3.37. Figure 3.38 shows an S200 Danaher drive unit. The Danaher drives and actuators are paired and communicate via a 'Smart Feedback Device' (SFD). This black box communication outputs the motor parameters to the drives and automatically sets up the drive with the specific characteristics of the motor. 


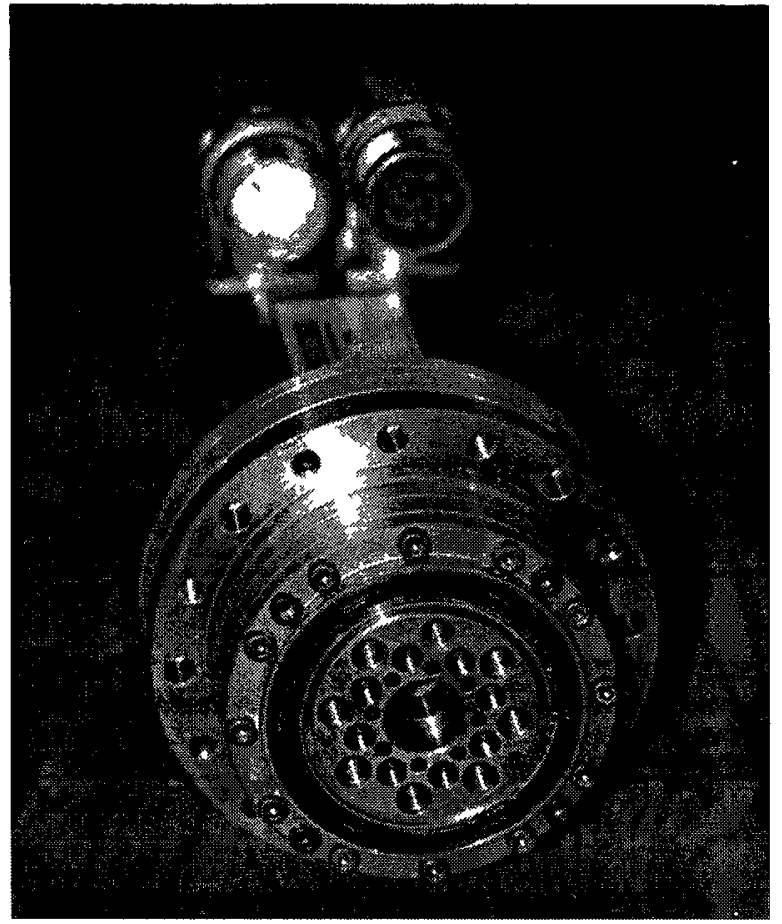

Figure 3.37: Joint 4 Assembled Actuator

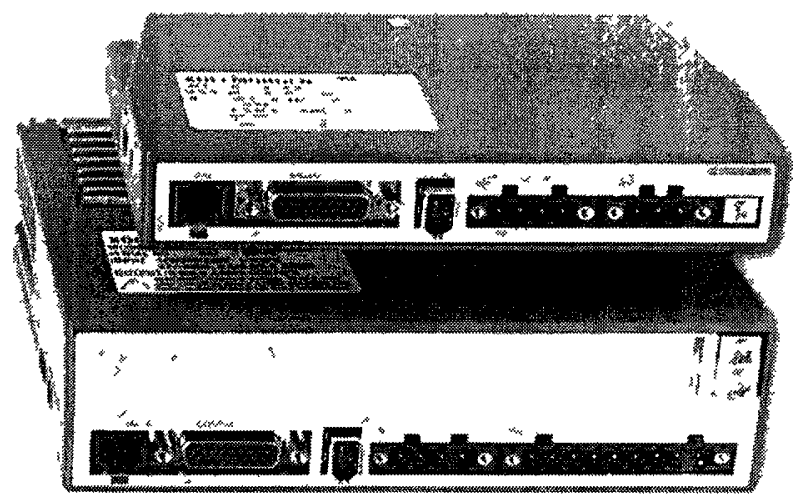

Figure 3.38: Danaher S200 Drive 


\subsubsection{Force Torque Sensor Selection}

The ATI Mini 85, 6 axis force torque sensor with SI-1900-80 calibration, in Figure 3.39, was selected using the forces and torques experienced by the foot during a gait cycle. The sensor includes a data acquisition signal processor and is interfaced with the DAQ card detailed below. The sensor ranges and specifications are found in [51] and [52], and are summarised in Table 3.9.The sensor weighs $0.635 \mathrm{~kg}$, has a diameter of $85 \mathrm{~mm}$ and a height of $30 \mathrm{~mm}$. This calibration has a force range that corresponds to the loading conditions of walking under full load. Due to this, the resolution under low loading conditions, such as manipulation of the platform by hand, the device cannot be made entirely transparent. A lower calibration would increased the resolution and would allow for more accurate low load manipulation of the end effector, however, the torques applied to the end effector during gait rehabilitation would saturate the sensor.

Table 3.9 Force Torque Sensor Specifications

\begin{tabular}{l|llll}
\hline & $\mathbf{F}_{\mathbf{x}}, \mathbf{F}_{\mathbf{v}}(\mathbf{N})$ & $\mathbf{F}_{\mathbf{z}}(\mathbf{N})$ & $\mathbf{T}_{\mathbf{x}}, \mathbf{T}_{\mathbf{v}}(\mathbf{N m})$ & $\mathbf{T}_{\mathbf{z}}(\mathbf{N m})$ \\
\hline Load Capacity & \pm 1900 & \pm 3800 & \pm 80 & \pm 90 \\
Resolution & $1 / 3$ & $5 / 12$ & $9 / 700$ & $2 / 350$ \\
Error (\%) & 1.5 & 1.0 & 1.25 & 1.25 \\
\hline
\end{tabular}

The calibration matrix for converting the analog voltages from the internal strain gauges to force data in $\mathrm{N}$ and $\mathrm{Nm}$ is:

$$
\begin{gathered}
\left\{\frac{F_{t}}{T_{t}}\right\}=C_{t} V_{t_{m}} \\
C_{t}=\left[\begin{array}{cccccc}
5.609 & -0.742 & -4.234 & 180.893 & 1.880 & -186.620 \\
2.563 & -214.831 & 2.510 & 105.190 & -6.322 & 180.442 \\
-239.440 & -3.775 & -234.178 & -1.427 & -239.572 & -3.096 \\
-0.043 & -0.032 & 7.687 & 0.041 & -7.783 & -0.092 \\
-8.981 & -0.086 & 4.358 & 0.013 & 4.424 & 0.048 \\
-0.049 & 4.921 & -0.074 & 4.827 & -0.031 & 5.019
\end{array}\right]
\end{gathered}
$$




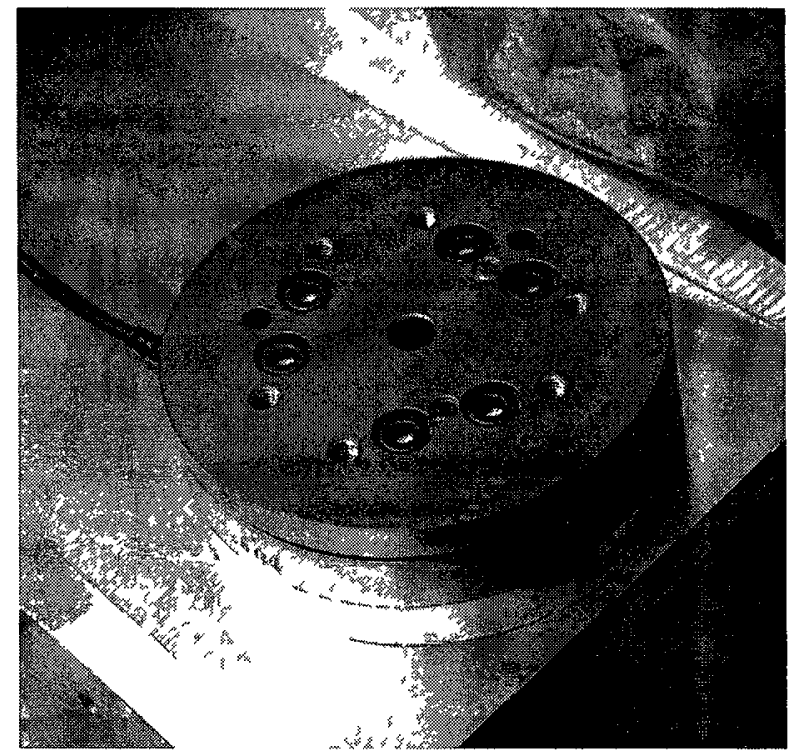

Figure 3.39 ATI Mini 85 F/T Sensor

\subsubsection{Controller Components}

The controller was designed on a host computer running MatLAB Simulink and QuaRC and compiled over a TCP/IP connection to the target PC running the hard real time operating system QNX. Quanser provided the QuaRC software, which compiles the real time code for the QNX machine as well as the data acquisition (DAQ) components. The DAQ is a Quanser Q8 card and breakout board. The board has 8 14-bit analog inputs, 8 12-bit analog outputs and 8 encoder inputs as well as 32 digital I/Os. Information from the F/T sensor, the Hall effect sensors and the actuators are input through the DAQ into the controller. Six of the eight analog in ports are used by the ATI Force/Torque sensor while the four analog outputs are used to control the actuators. The digital ins and outs are used to control the safety features, such as the Hall proximity sensors and the emergency stop buttons. The encoder inputs are taken from the S200 drives. Additional information on the Quanser components is provided in [53] and [54] 


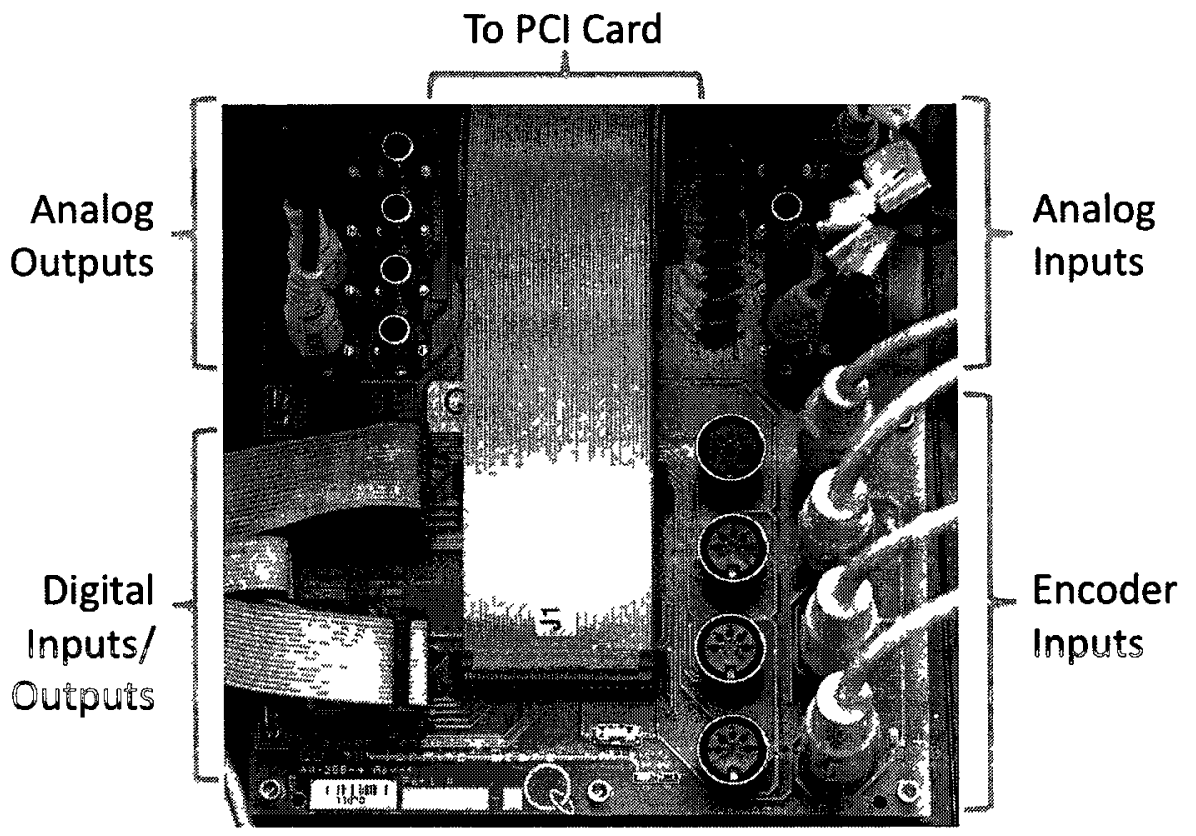

Figure 3.40: Q8 Breakout Board

\subsection{Chapter Summary}

This chapter outlined the development of the platform. Gait analysis was performed to determine the loading requirements of the manipulator. The configuration of the platform was motivated and a clear set of design requirements was determined. From these requirements the initial design of the manipulator was performed, determining the optimized design parameters and the selection of the actuation components. Figure 3.41 shows the flow of information through the design. The next chapter presents the detailed design of the manipulator and the structural analysis. 


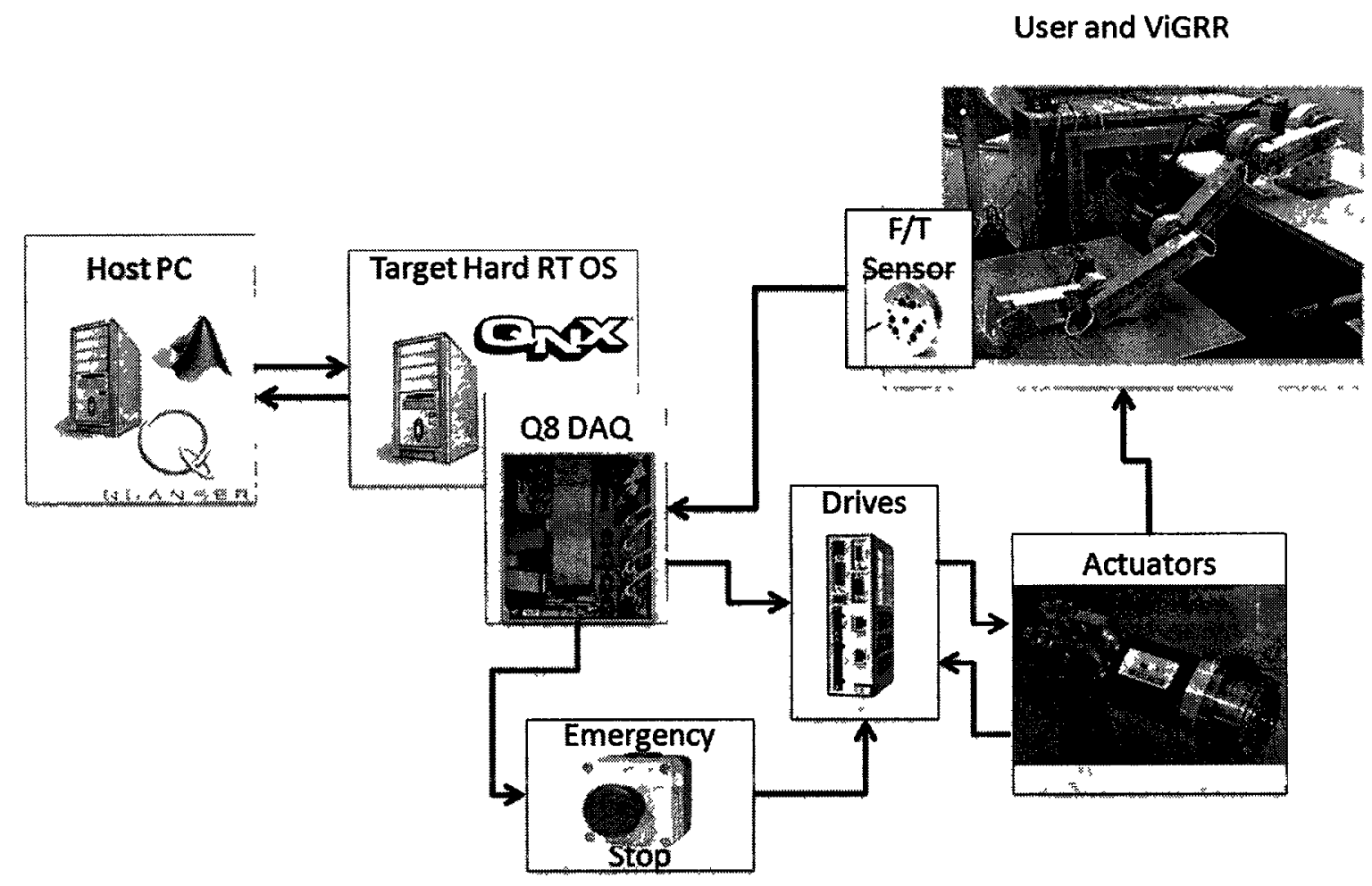

Figure 3.41: Layout of Robotic Apparatus 


\section{Chapter 4: Design and Structural Analysis of Virtual Gait Rehabilitation Robot Manipulator}

This chapter builds upon the design requirements and preliminary design presented in Chapter 3: and develops the detailed design of various components of the platform, including the design of the links, the joints, material selection and optimization, and the end effector and safety features design. The chapter ends with the structural analysis of the final design and design verifications. The structure of ViGRR was designed using Pro/Engineer Wildfire 4 and the structural analysis was completed using the accompanying Pro/Mechanica suite.

All components were manufactured in the Carleton University Mechanical and Aerospace Engineering Machine shop using both Computer Numerically Controlled (CNC) and manual machines. Figure 4.1 shows the finished and assembled design with the hand manipulator attached at the end effector. The patient would be seated alongside the manipulator on the desk and the foot would be attached to the end effector. The wiring that is visible in the foreground of the robot is for the Hall Effect limit sensors. Figure 4.2 details the design process of the manipulator design as well as showing the design optimization loops that were followed through the design process. 


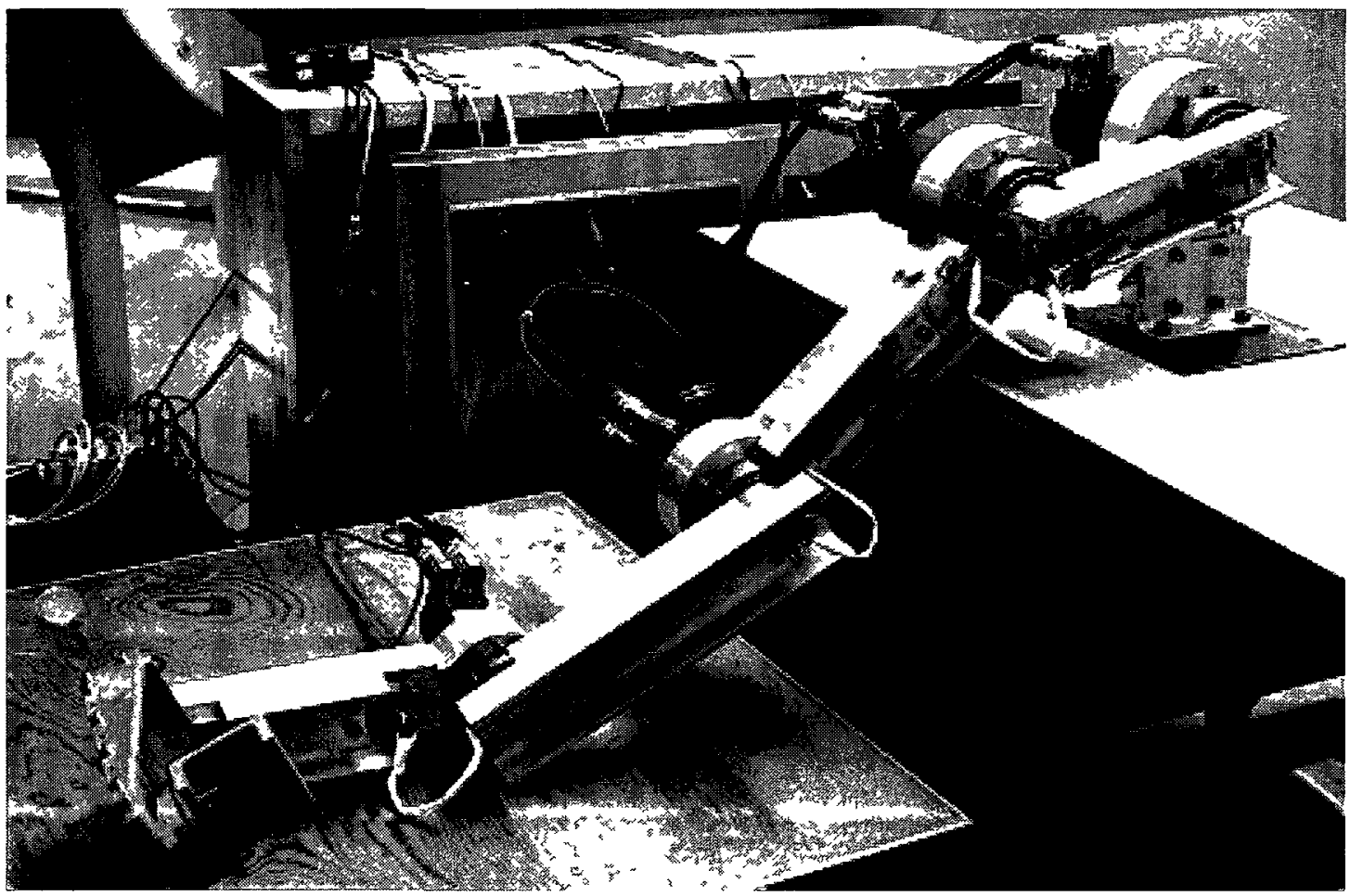

Figure 4.1: ViGGR Manipulator

\section{11 Link Design}

ViGRR, as a prototype platform, had a number of conflicting design criteria that played into the development of the manipulator. Though stiffness of the end effector was paramount, decreasing weight, cost and manufacturing time were also factored into the design choices. Only common materials and cross sections were considered in order to decrease the cost and time spent during fabrication in the machine shop.

\subsubsection{Material Selection}

For all machined parts, 6061 T6 Aluminum was used, including linkages, joint components and end effector. Table 4.1outlines the various materials considered. Aluminum 6061 was chosen due to its reasonable strength to weight ratio, low cost, high availability and machinability compared to the other materials. Stainless steel fasteners 
were used, either SAE 301 or 316 depending on availability due to their high corrosion resistance.

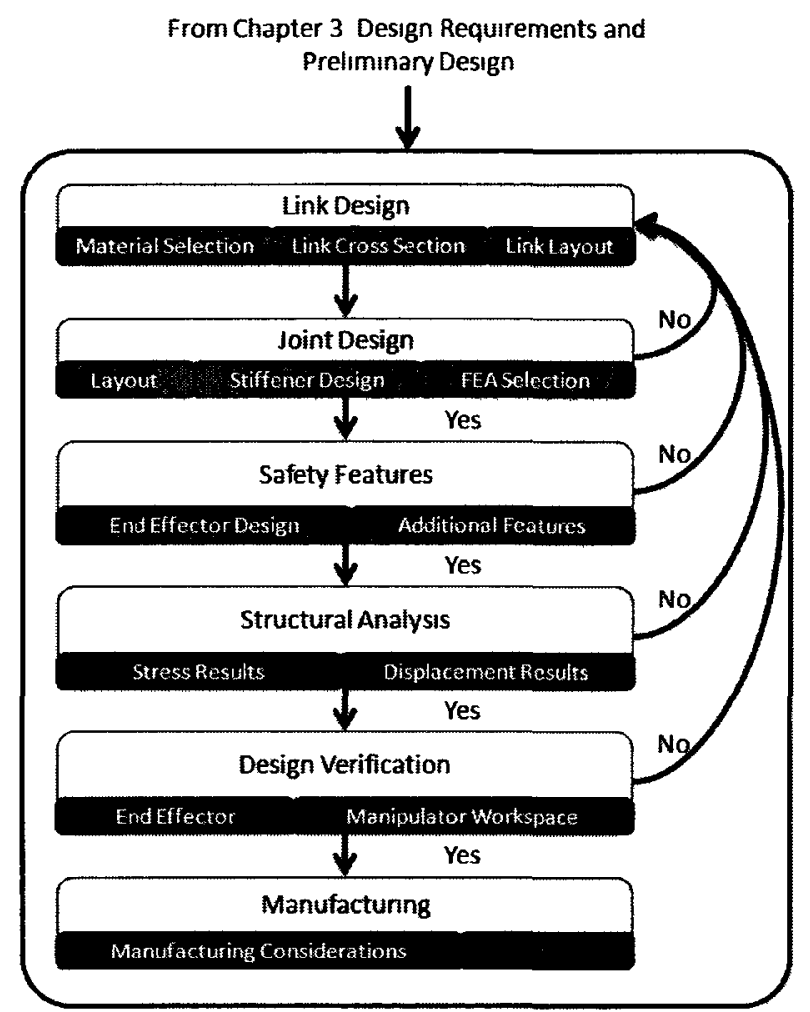

Figure 4.2: ViGRR Structural Design Process

Table 4.1 Material Considered in Design Process

\begin{tabular}{l|llll}
\hline Material & $\begin{array}{l}\text { Strength } \\
\text { Weight }\end{array}$ & Availability & Cost & Machinability \\
\hline \hline Al 6061 T6 & Med & High & Low & $50 \%$ \\
Al 7075 T6 & Med & Low & Med & $70 \%$ \\
Al 2024 T6 & Low & Med & Low & $70 \%$ \\
HS Steels & Med & Med & Low & $80 \%$ \\
Composities & High & Med & High & $\sim 0$ \\
\hline
\end{tabular}




\subsubsection{Link Cross Section Selection}

Prior to the final link lengths and loading conditions being determined, an approximation of the load at the end effector was used to determine the cross section of the linkages. A load of $950 \mathrm{~N}$ in the $\mathrm{X}$ direction and $250 \mathrm{~N}$ in the negative $\mathrm{Y}$ direction were applied to a four link configuration with an overall length of 1.3 meters for the analysis. These force values correspond to the ground reaction forces presented at the end of Section 3.1 while the final loading conditions take into account the robot dynamics and the necessity to support the leg while the patient is in a horizontal position. The final configuration presented in Chapter 3 is an overall length of 0.971 meters and loads of 1315.4 and 234.2 in the $\mathrm{X}$ and $\mathrm{Y}$ directions respectively. Figure 4.3 depicts the setup that was analyzed using Pro/Mechanica and Table 4.2 lists the results for the various cross sections tested. Though Harmonic Drives are quite flexible, to analyze the structural stiffness the interfaces between the links were modelled as rigid and the load was applied to a plate offset to the side of the manipulator to simulate the approximate location of the foot interface. 


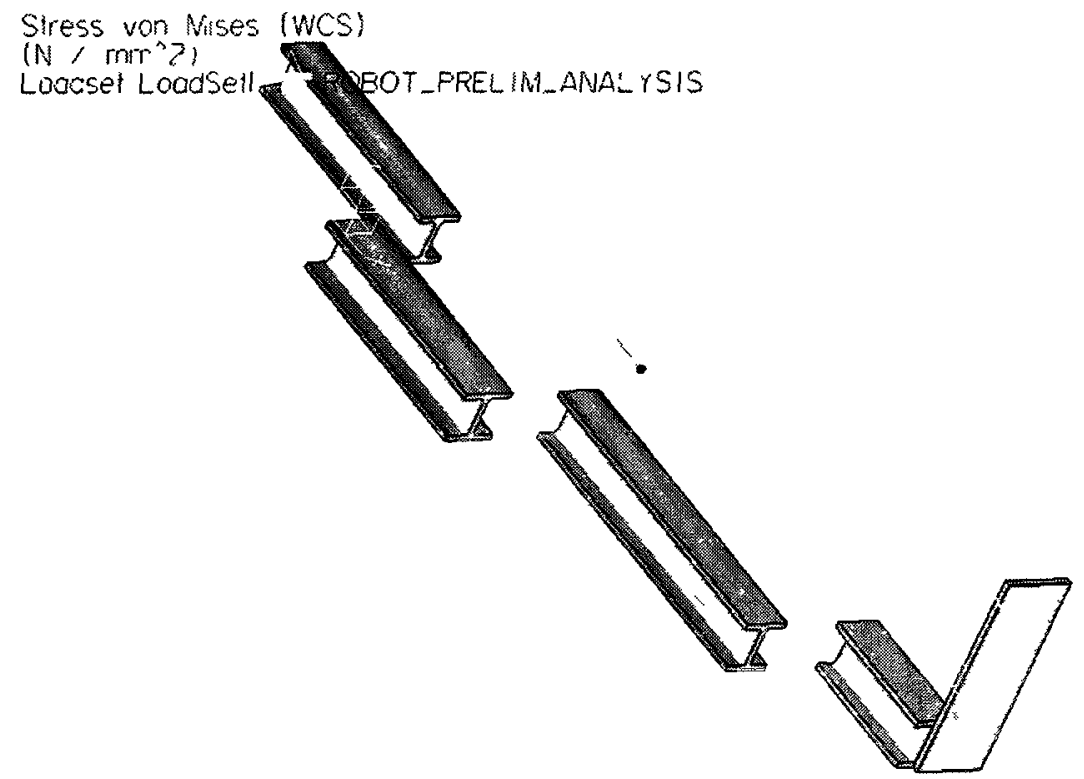

$7.416 e+01$

$65020+01$

5. -68es01

$4944 e+01$

$4.120+01$

3. $296 e+01$

2. $472 e+01$

$1648 e+01$

9. $248 \mathrm{e}+00$

Figure 4.3: 1 Beam Analysis

Table 4.2: Results of Cross Section Analysis

\begin{tabular}{rllll}
\hline Cross Section & $\begin{array}{l}\text { Dimensions } \\
\text { Height x Width } \times \\
\text { Thickness }(\mathbf{m m})\end{array}$ & $\begin{array}{l}\text { Mass per } \\
\text { length } \\
\mathbf{( k g / m )}\end{array}$ & $\begin{array}{l}\text { Deflection } \\
(\mathbf{m m})\end{array}$ & $\begin{array}{l}\text { Maximum } \\
\text { Stress } \\
(\mathbf{M P a})\end{array}$ \\
\hline $\begin{array}{r}\text { Rectangular Tube } \\
\text { Rectangular Tube }\end{array}$ & $76.2 \times 101.6 \times 6.35$ & 5.66 & 3.70 & 19.1 \\
(Rotated) & $101.6 \times 76.2 \times 6.35$ & 5.66 & 5.10 & 27.3 \\
Square Tube & $63.5 \times 63.5 \times 6.35$ & 3.92 & 9.02 & 40.2 \\
I Beam & $76.2 \times 63.5 \times 8.86$ & 4.44 & 13.9 & 77.4 \\
Channel Section & $101.6 \times 63.5 \times 6.35$ & 3.70 & 14.9 & 82.4 \\
\hline
\end{tabular}


Though highly stiff, the rectangular cross section would have placed increased load on the actuators due to the increased mass of the sections. The channel cross section caused significant rotations of the end effector location due to the eccentric loading on a non symmetric cross section.

The I-beam cross section was chosen as the link design as it is a light weight cross section that offers high bending and torsional stiffness. The design also allows for the cross section to overlap the joint center while minimizing the offset distance between the links.

As the manipulator articulates alongside the patient's leg, the actuators had to face out, away from the patient, see Figure 4.4. To prevent a large space between the patient and the base of the manupulator two of the motors had to be placed on the second link, leading to a high mass concentration and a high level of inertia for link 2, Figure 4.5, while the masses and inertias of links 1 and 3 were minimal with no actuator masses. The second design would have a slightly different workspace as the chosen design since the fourth link would not be impeded, though this is not a significant advantage as the end effector orientation does not vary significantly during gait rehabilitation.

The mass properties of the four links are shown in Table 4.3. The I-beam links were used as is and overlapped at the joints to improve stiffness. Material was removed in order to accommodate the other components. Figure 4.6 shows the two link styles, depending on whether the link is attached to the Harmonic Drives or to the joint design.

Table 4.3: Estimated Mass Properties of Links from CAD Model

\begin{tabular}{|c|c|c|c|}
\hline Link n & $\begin{array}{l}\text { Mass } \\
(\mathbf{k g})\end{array}$ & $\begin{array}{l}\text { Center of Mass } \\
\qquad(x, y, z) \\
\text { from Joint } n(m)\end{array}$ & $\begin{array}{c}\text { Inertia } I_{\mathrm{zz}} \\
\left(\times 10^{-2} \mathrm{kgm}^{2}\right)\end{array}$ \\
\hline 1 & 1.16 & $(0.1308,0,0.00087)$ & 2.777 \\
\hline 2 & 13.51 & $\begin{array}{c}(0.1112,0.0017 \\
0.0150)\end{array}$ & 51.693 \\
\hline 3 & 1.80 & $(0.1155,0,0.0404)$ & 4.498 \\
\hline 4 & 4.95 & $\begin{array}{c}(0.0686,0.00778 \\
0.0539)\end{array}$ & 5.782 \\
\hline
\end{tabular}


(2)
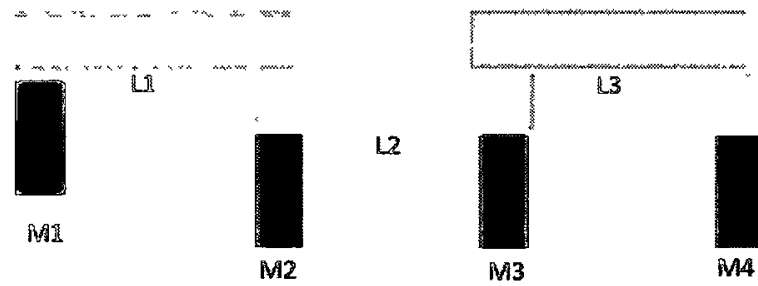

L4

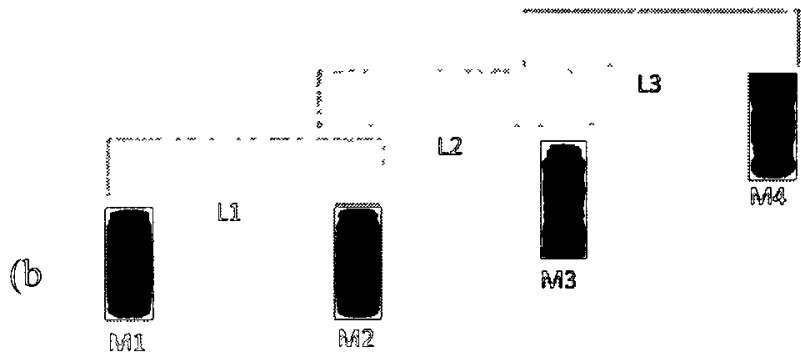

14

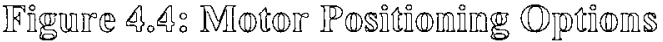

(a) Selected Layour

Desigm A

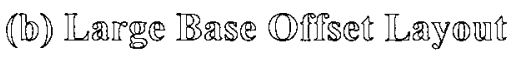

Desigm BB

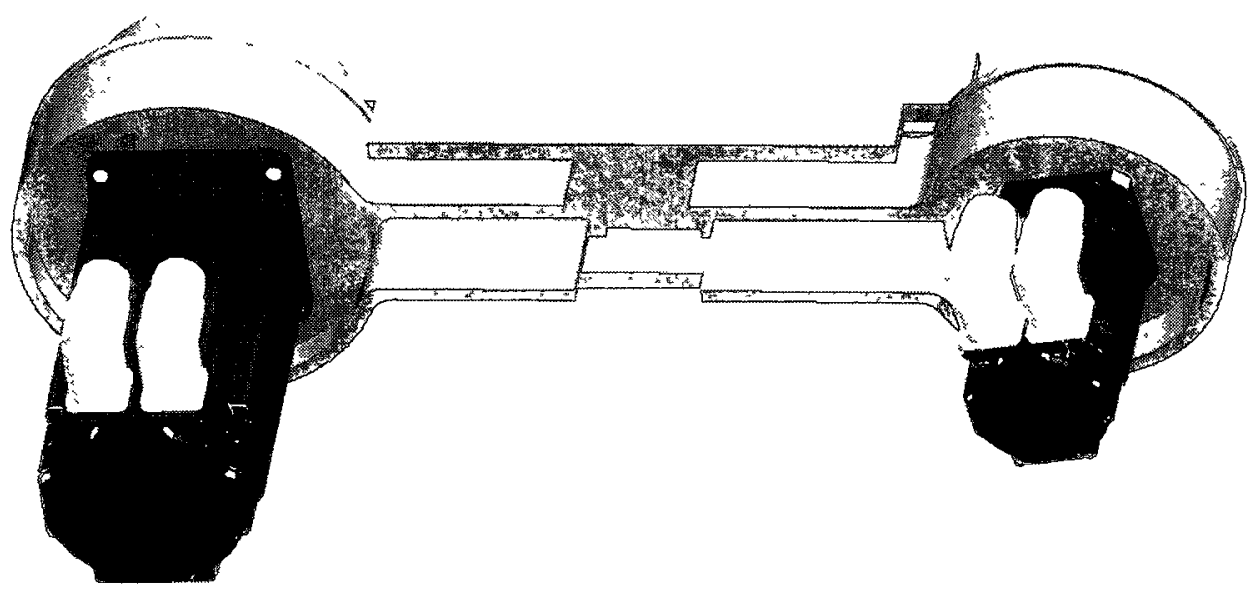

Figure 4.5: Link 2 with Actuators installed 


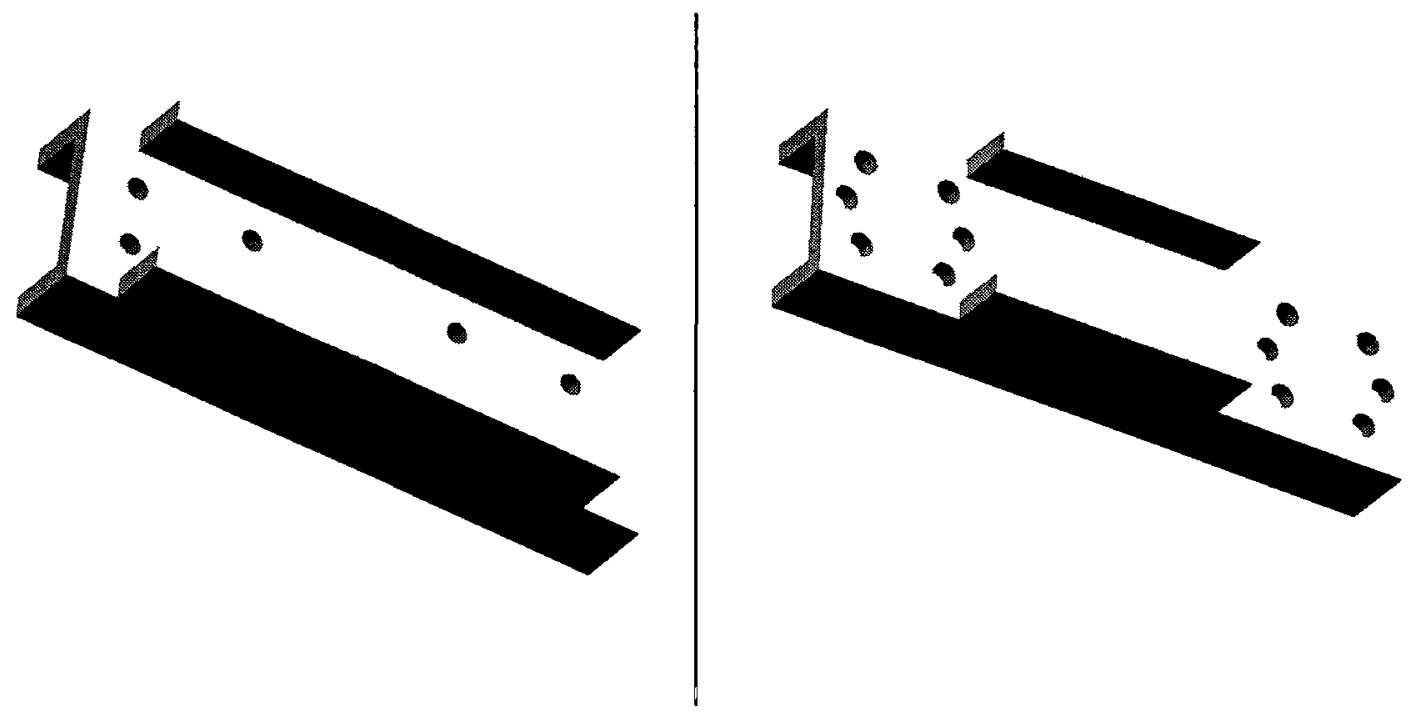

Figure 4.6: Link Designs

\subsection{Joint Design}

The joint design connects one link to the next and houses the actuation components. This creates a location where there are high loads in the structure creating significant flexion in the joints.

The joints present a location of significant structural flexibility in the design of a serial manipulator. The joint design attaches on one end to the I-beam link through a 4 hole bolt pattern. The other link is attached directly to the output face of the Harmonic Drive. The joint part mates to the Harmonic Drive and motor while supporting the structure with a circular ring.. Due to alignment of the links, in order to increase the range of motion of the joints, inserts were used to offset the links from the face of the Harmonic Drive. In order to determine a suitable design for the stiffener the Computer Aided Design (CAD) model of the manipulator was analyzed iterating the design and dimensions of the design. The final design is shown in Figure 4.7. 


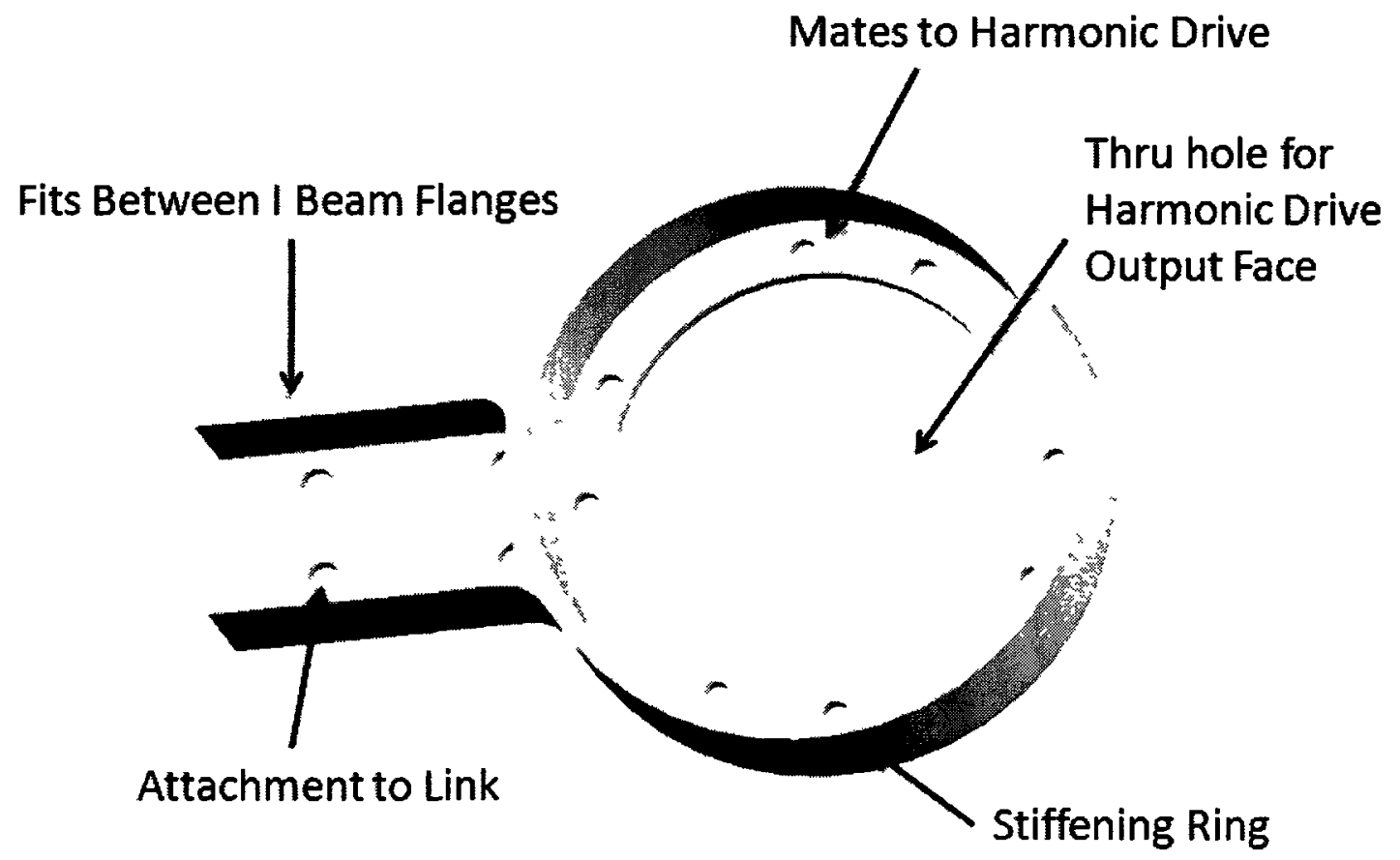

Figure 4.7: Final Joint 2 Stiffener Design

Numerous designs were analyzed and the final design was determined by using the Computer Aided Design (CAD) model of the manipulator and loading a variety of joint designs in order to determine an effective design. Figure 4.8 depicts the various designs of the stiffening section of the joint, including the links and the Harmonic Drives, the motors were removed to add clarity. Table 4.4 outlines the results of each design, showing both the maximum stress in the manipulator and the deflection of the end effector. 
Table 4.4: FEA Results of Various Joint Designs

\begin{tabular}{ccc}
\hline Joint Design (Figure 4.5) & Max Stress & Max Deflection \\
\hline (a) & $590 \mathrm{MPa}$ & $32 \mathrm{~mm}$ \\
(b) & $548 \mathrm{MPa}$ & $23.3 \mathrm{~mm}$ \\
(c) & $541 \mathrm{MPa}$ & $22.8 \mathrm{~mm}$ \\
(d) & $472 \mathrm{MPa}$ & $21.5 \mathrm{~mm}$ \\
(e) & $485 \mathrm{MPa}$ & $18.3 \mathrm{~mm}$ \\
(f) & $467 \mathrm{MPa}$ & $17.3 \mathrm{~mm}$ \\
\hline
\end{tabular}

The chosen stiffening design was then iterated to determine suitable wall heights and thicknesses while also taking into consideration the machinability of the part on a three axis CNC mill. The final joint desıgn has a stıffener thıckness of $6.35 \mathrm{~mm}$ and a stiffener height of $50.8 \mathrm{~mm}$. Figure 4.7 shows the final layout of the joint design for joint 2 , including the bolt hole patterns for attaching to the CSG 32 Harmonic Drive and the I Beam link.

Table 4.5: Joint Design Dimensions

\begin{tabular}{cc}
\hline & $\begin{array}{c}\text { Selected Design } \\
\text { Dimensions }\end{array}$ \\
\hline Wall Thickness & $6.35 \mathrm{~mm}$ \\
Height & $50.8 \mathrm{~mm}$ \\
Length of Flange & $76.2 \mathrm{~mm}$ \\
\hline
\end{tabular}




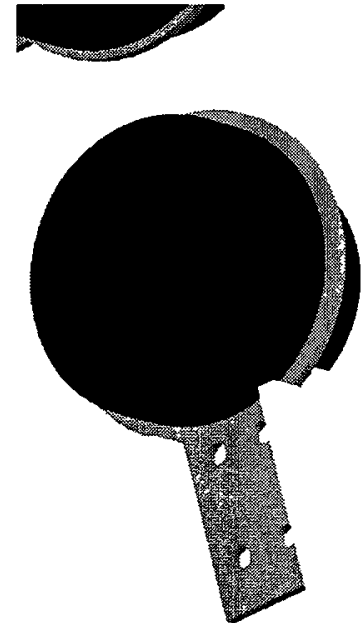

(a)

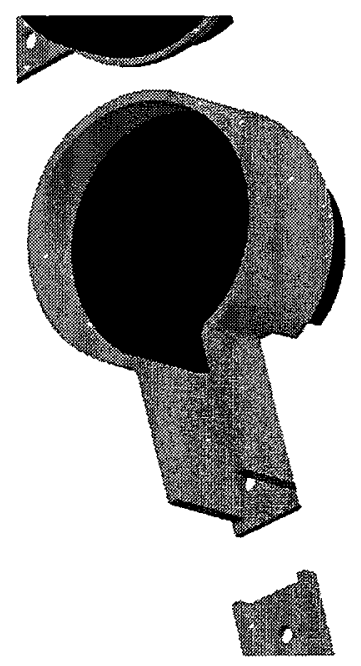

(d)
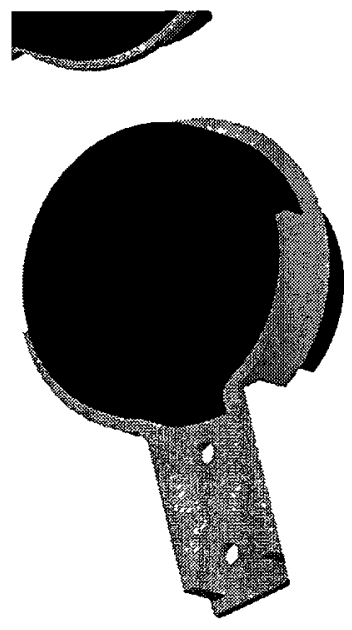

(b)
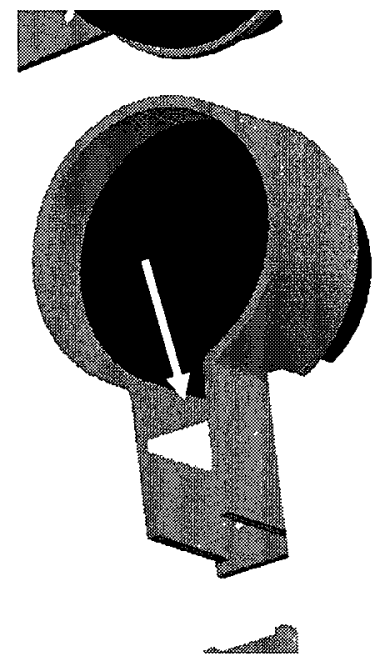

(e)
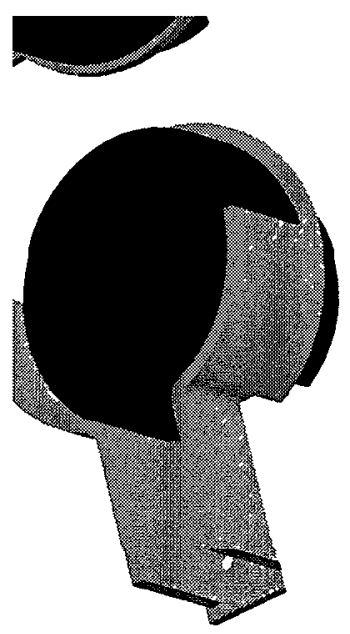

(c)

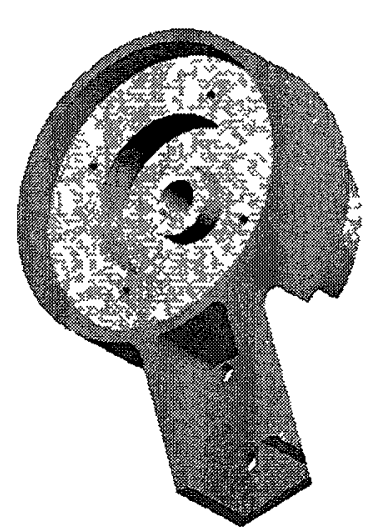

(f)

Figure 4.8: Joint Design Evolution

(a) Flat Plate (b) Partial Stiffener (c) Tall Partial Stiffener (d) Full Ring

(e) Ring with Supporting Fin (light section) (f) Single Structure Full Ring Support 


\subsection{Safety Features}

In the event of an error in the operation of the system due to a controller malfunction, or other unforeseen circumstances, the primary concern is the safety and wellbeing of the user. Section 4.4.1 discusses the design of the foot attachment device and Section 4.4.2 outlines the various other features that are implemented to decrease the risk of injury.

\subsubsection{End Effector Design}

During the optimization of link lengths, the offset angle of the end effector was also specified to minimize the demand on the actuators. The optimal offset angle was determined to be 33.5 degrees off the perpendicular, Figure 4.9. This figure also shows the joint, end effector and sensor coordinate systems as they were used throughout the design of the platform and controller. The end piece was overdesigned so that there was no added deflection to the end effector position, as the foot plate must be rigid in order to synthesize the sensation of gait in a haptic manipulator. In the case where there is an instability in the controller or a situation where the configuration of the manipulator could cause injury to the patient the foot must be able to release. Two methods were installed in serial to mitigate the chance of injury and allow the foot to be released from the manipulator.

\section{Magnetic Lock}

Attached directly to the ATI sensor is a Securitron MCL-24 Magnetic Lock with a $90 \mathrm{~kg}$ holding force, depicted in Figure 4.10. The electromagnet is powered by a 24 Volt DC power supply and uses an electronic switch connected to the DAQ which is triggered by the various safety features listed below. The close proximity of the electromagnet to the Force Torque sensor does not affect the sensor. No discernable increase is evident in the filtered or unfiltered sensor data. Figure 4.11 to Figure 4.16 show the filtered noise data from the sensor with the magnet both enabled and disengaged. 


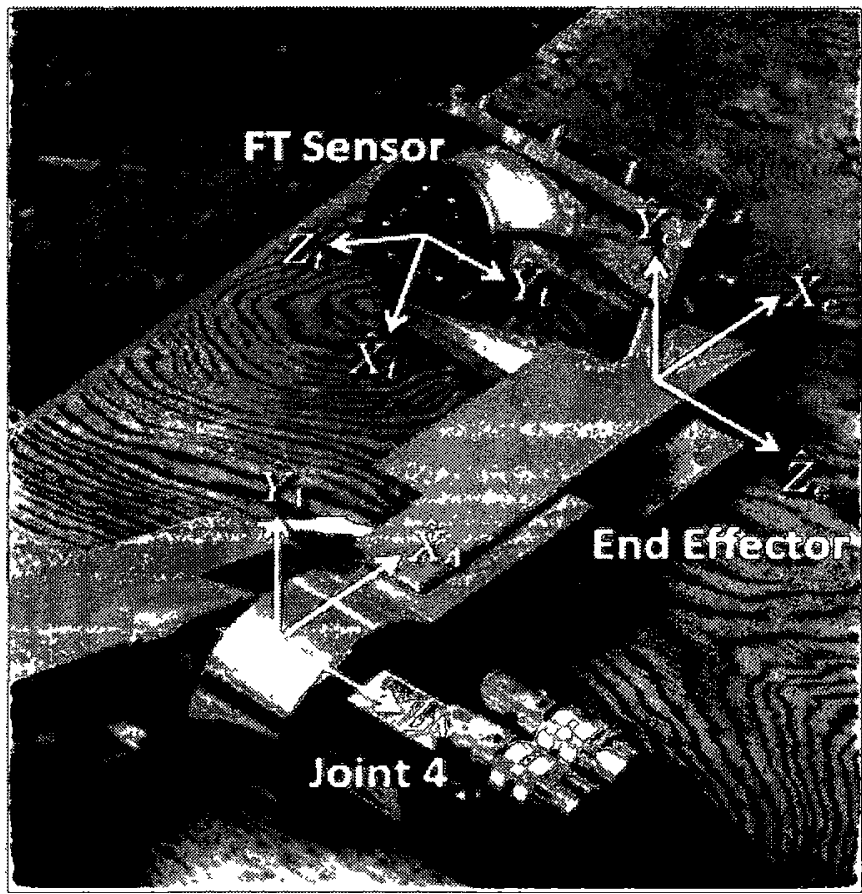

Fingre d.9: End Efiector Angle and Coordinate System

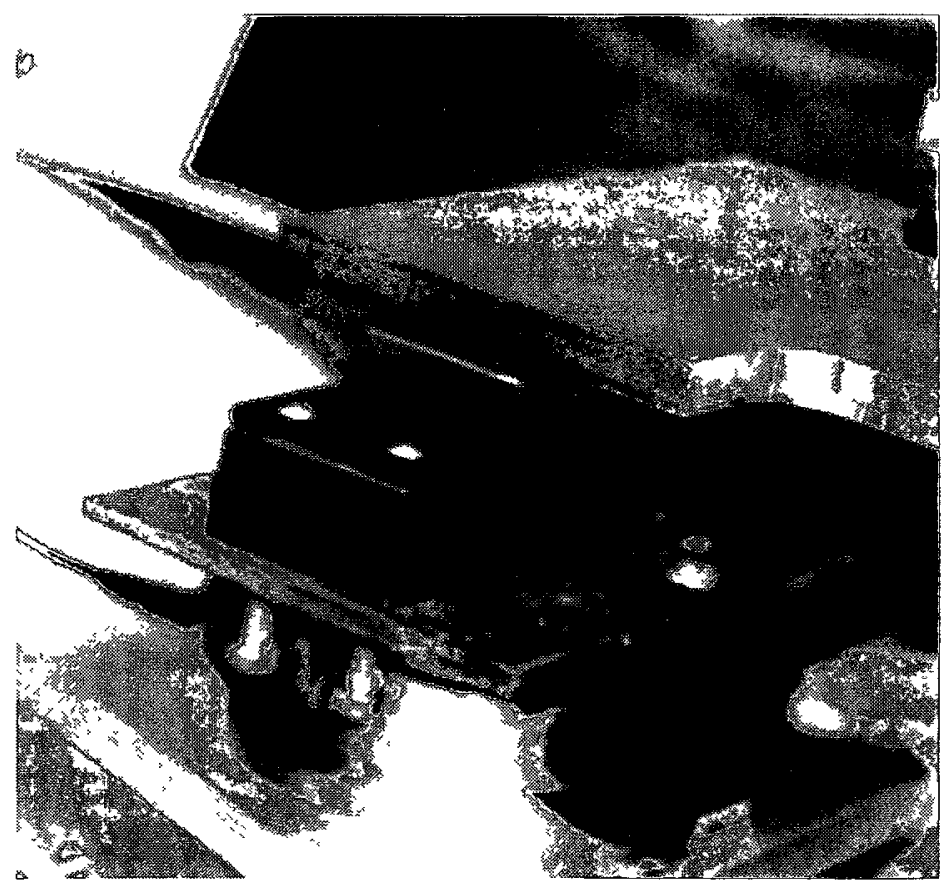

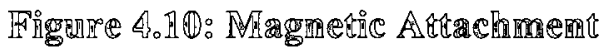




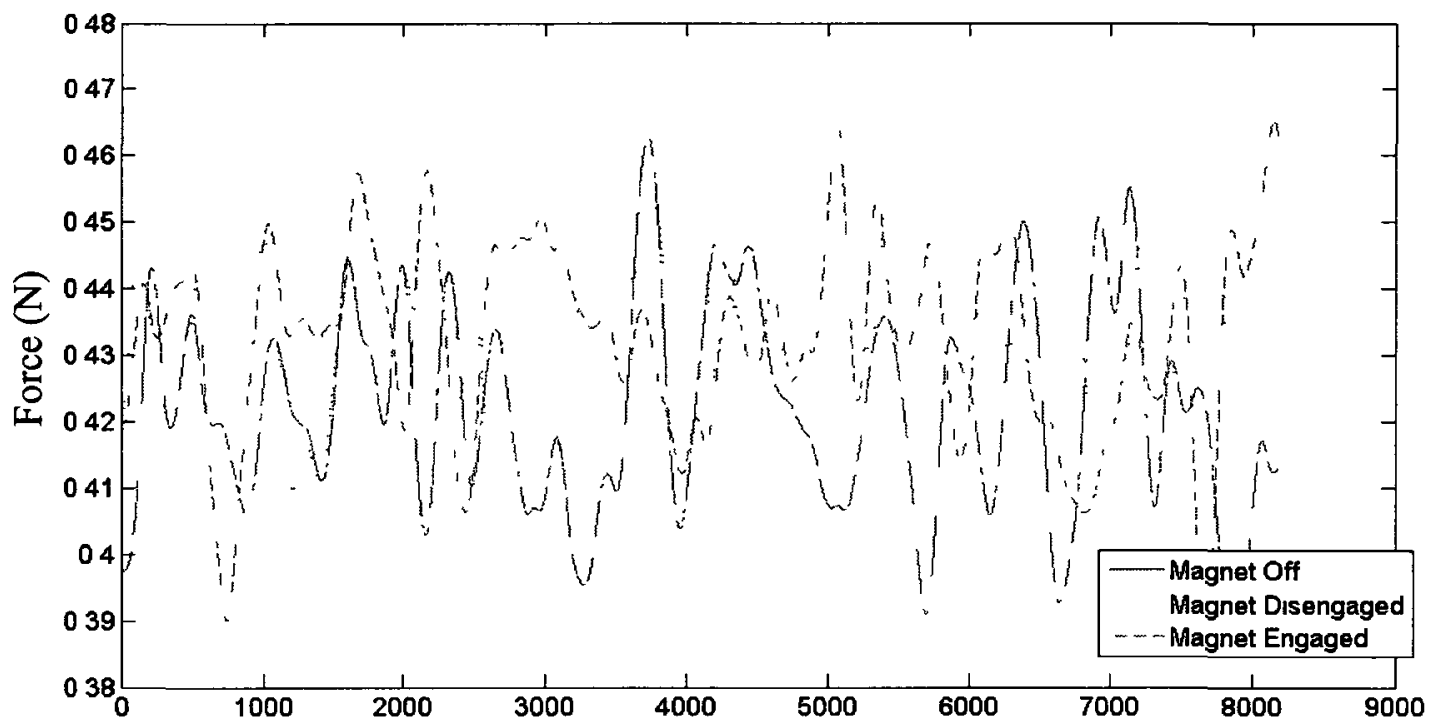

Figure 4.11: Filtered Force Torque Sensor Force Noise in X Direction

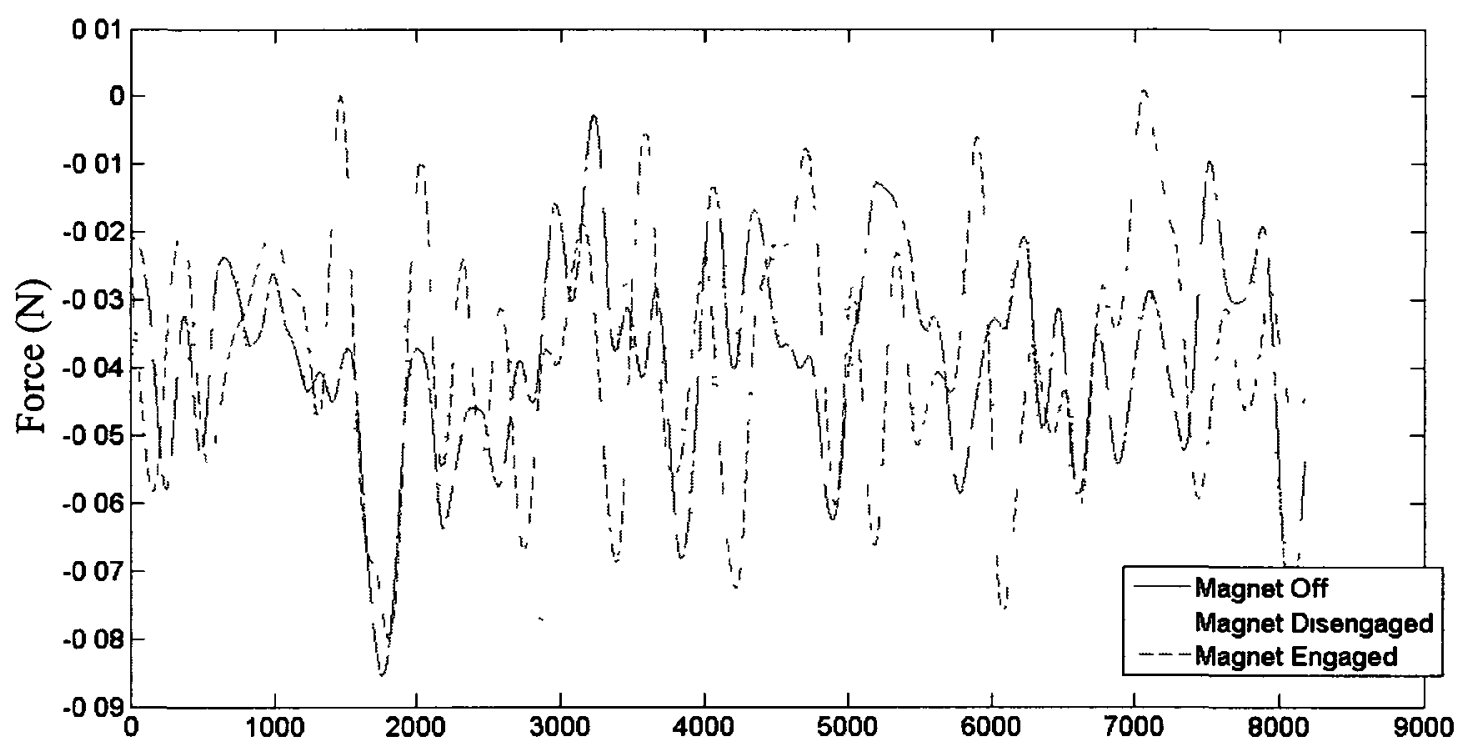

Figure 4.12: Filtered Force Torque Sensor Force Noise in Y Direction 


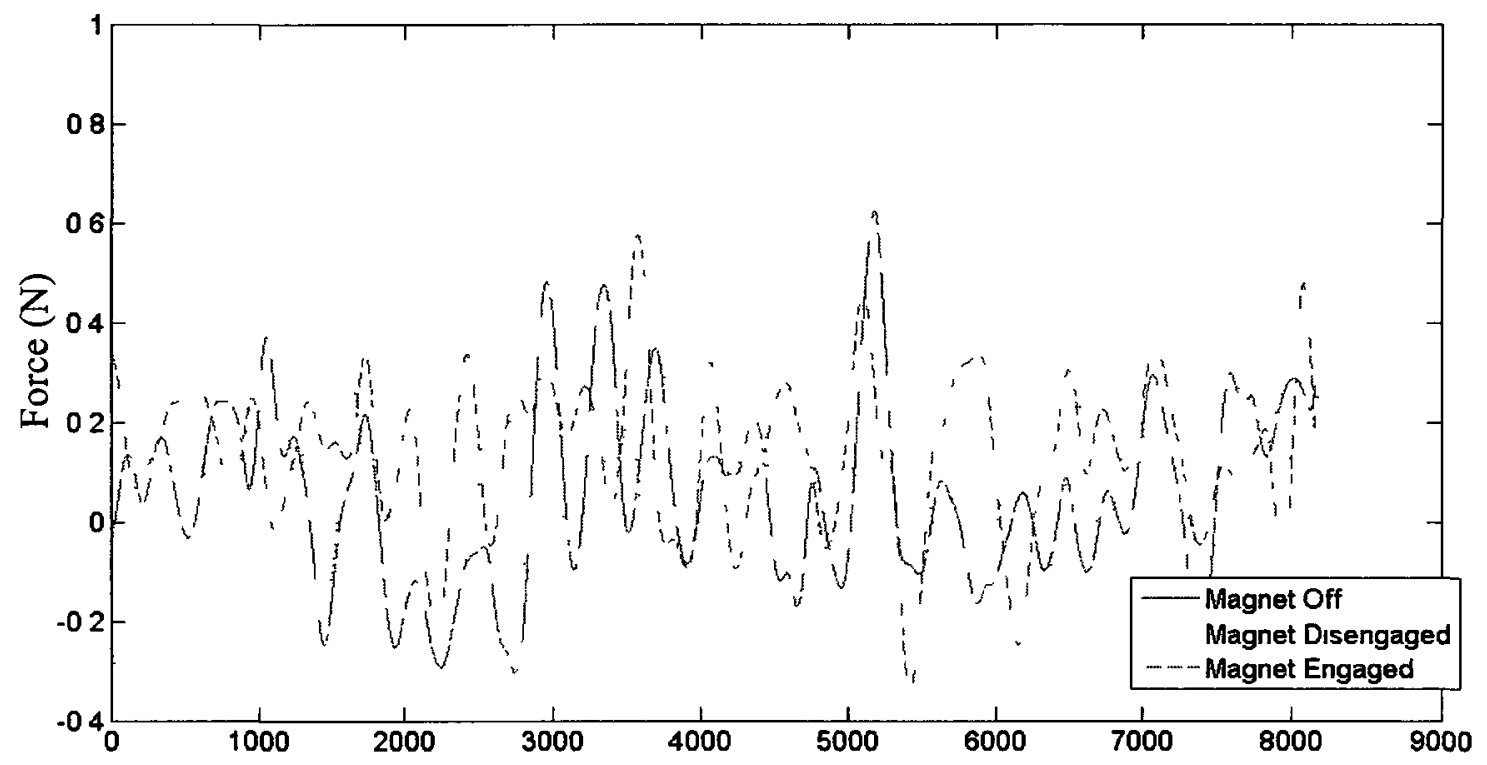

Figure 4.13: Filtered Force Torque Sensor Force Noise in $Z$ Direction

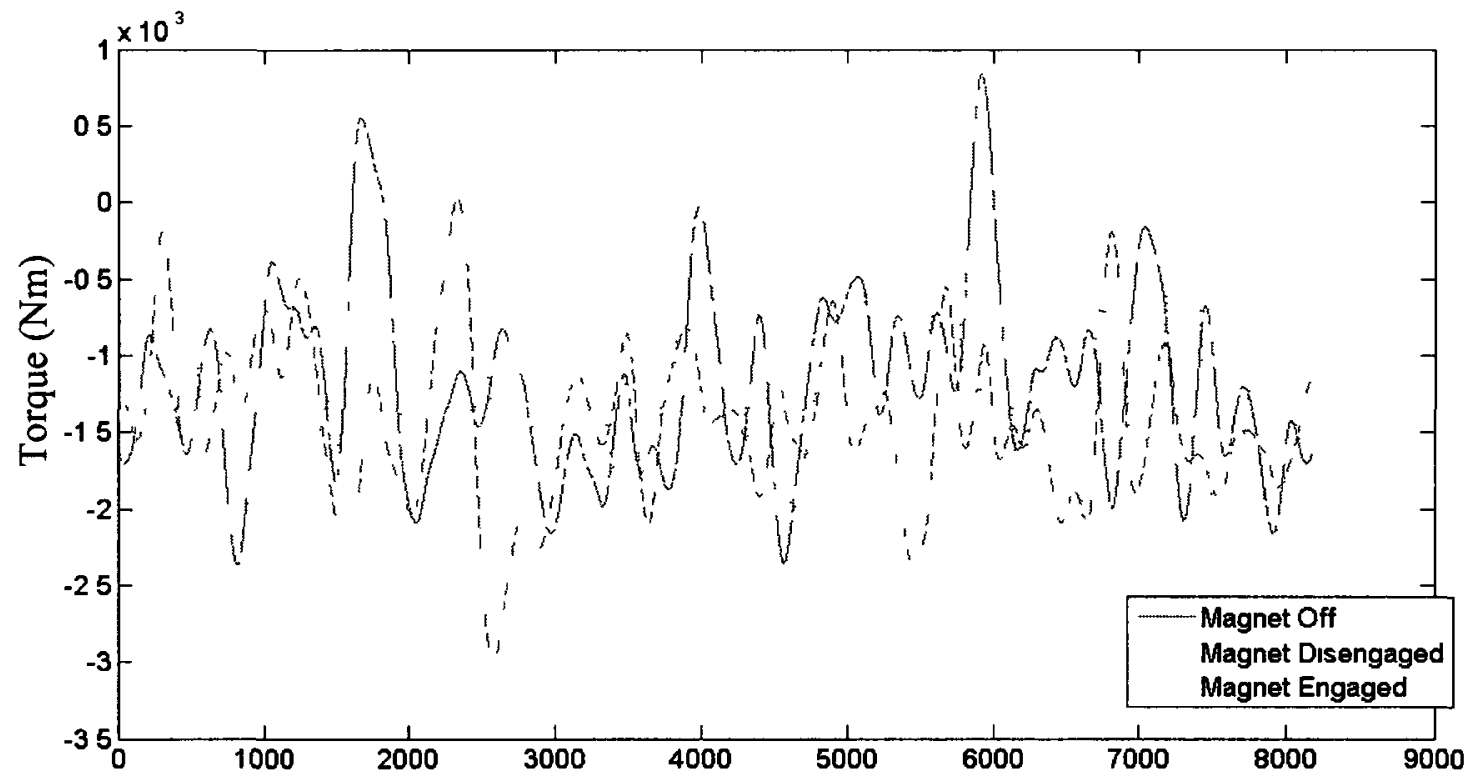

Figure 4.14: Filtered Force Torque Sensor Torque Noise about X Axis 


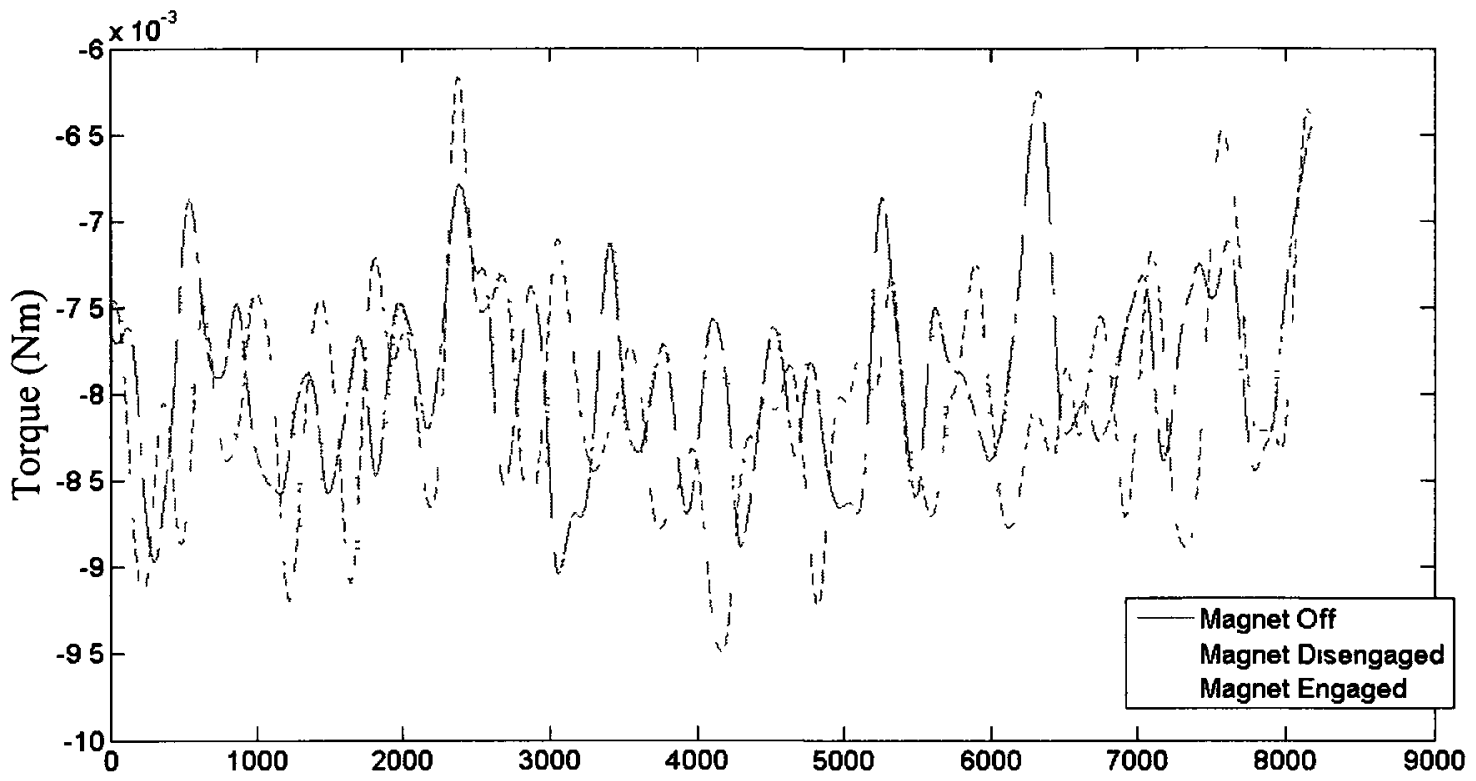

Figure 4.15: Filtered Force Torque Sensor Torque Noise about Y Axis

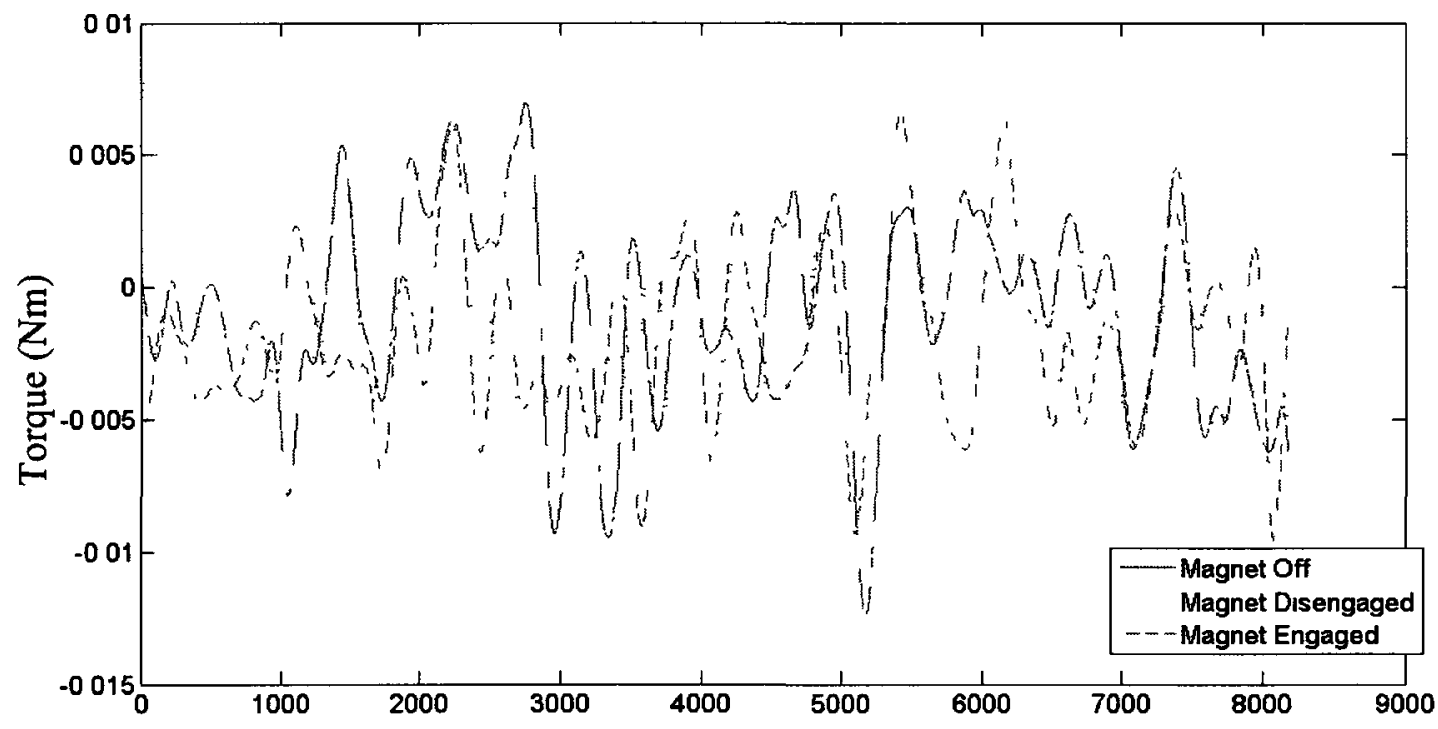

Figure 4.16: Filtered Force Torque Sensor Torque Noise about $Z$ Axis 


\section{Preloaded Mechanical Release}

Attached to the magnetic lock is a mechanical fuse designed to release under extreme loading conditions. The fuse contains two springs that are pretensioned to allow release of the device above the pretensioned load. The springs are placed in tandem so that the device will decouple at two different loads, a higher load in compression than in tension.

To prevent the manipulator from moving too close to the patient, into an incompatible location, an adjustable crash bar compresses the spring assembly, allowing the patient to detach their foot. The system is held in compression by the screw components that are attached to the patient's foot, shown in Figure 4.11. As the two outer plates on the right are compressed by the bolts, the load is transferred to the spring on the right, the more compliant of the two. This spring is disengaged through tension, further compressing the spring and decoupling the top plate of the foot assembly from the spring system. The crash bar makes contact with the system above the screw/spring assembly on the left hand side of Figure 4.17 and further compresses the high tension spring, decoupling the lower plates. However, in the current design the magnet represents the weakest attachment location, under torque from the end effector design, though a higher capacity magnet is recommended for future work. The foot plate is attached to the mechanical fuse and hook and loop strapping around the top of the foot and across the heel holds the foot in place.

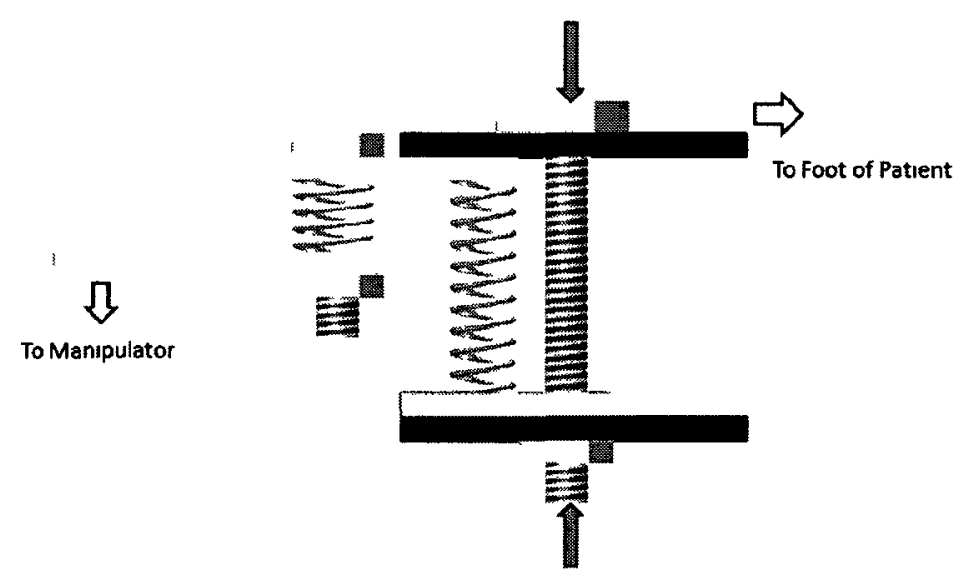

Figure 4.17: End Effector Design 
Figure 4.18 shows the layout of the end effector design. Part 1 is the force torque sensor, 2 , the electromagnet, is attached to the sensor using a interface plate. The strike plate is magnetically attached to the magnet and attaches to the rest of the joint assembly. Part 3 is the stiff spring which is activated in compression of the system, while 4 is compressed further when the user pulls against the motion of the manipulator to decouple the foot. Part 5 is the foot attachment plate and the two rods numbered Item 6 are guide rods used to keep the assembly aligned. Part 7, the two screws, tighten the assembiy and pretension the springs.

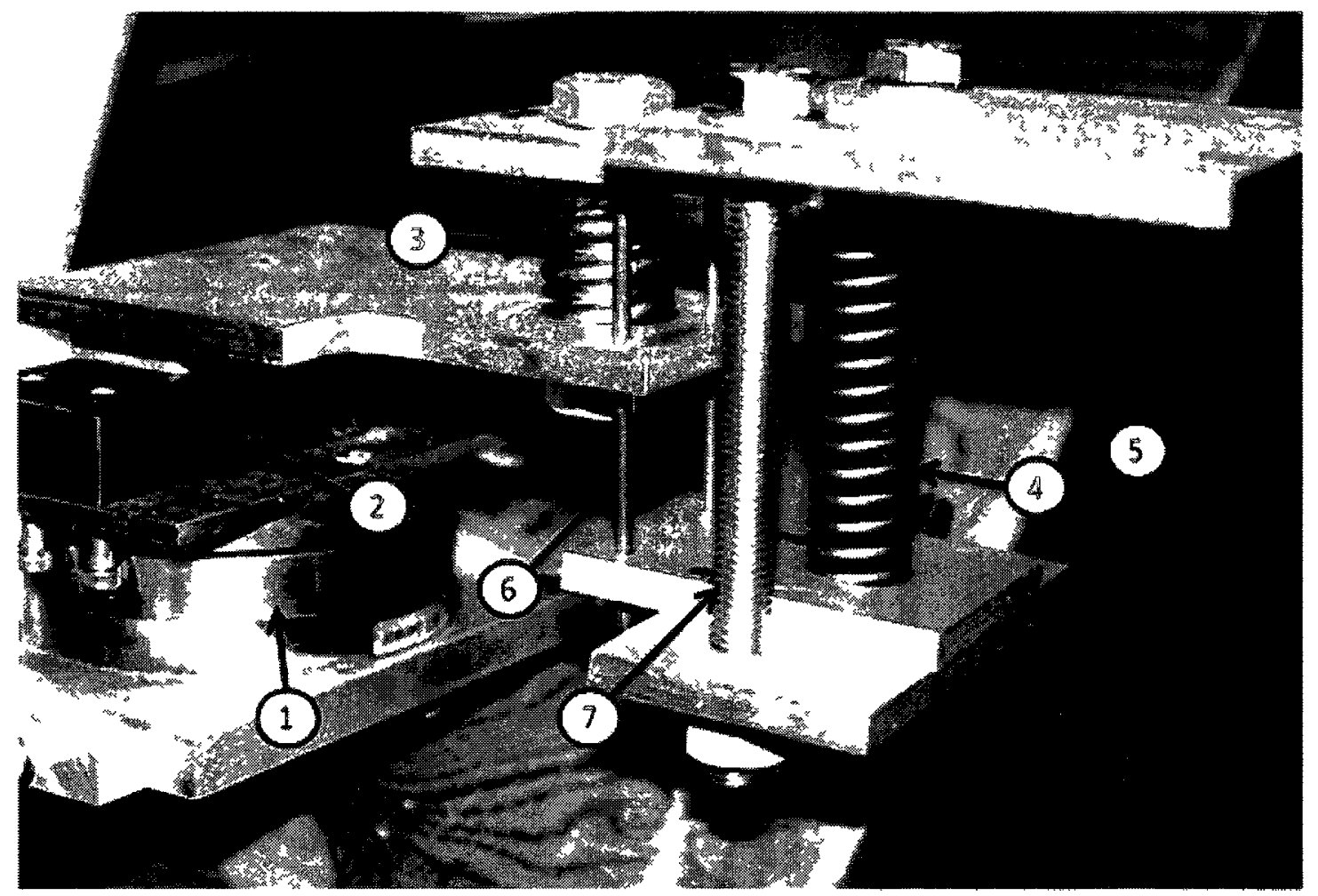

Figure 4.18: Picture of Spring Device 


\subsubsection{Remaining Safety Features}

\section{Controller Safety Limits}

To prevent the manipulator from becoming unstable and doing damage to the components or the user the limits in Table 4.6 were put in place.

Table 4.6: Controller Software Safety Limits

\begin{tabular}{|c|c|c|c|c|}
\hline & Parameter & Minimum Limit & Maximum Limit & Units \\
\hline \multirow{3}{*}{ Joint 1} & Position & -0.2 & 3.288 & $\mathrm{rad}$ \\
\hline & Velocity & -2.2 & 2.7 & $\mathrm{rad}$ \\
\hline & Torques & -250 & 250 & $\mathrm{Nm}$ \\
\hline \multirow{3}{*}{ Joint 2} & Position & -2.26 & 2.23 & $\mathrm{rad}$ \\
\hline & Velocity & -2.5 & 2.5 & $\mathrm{rad}$ \\
\hline & Torques & -200 & 200 & $\mathrm{Nm}$ \\
\hline \multirow{3}{*}{ Joint 3} & Position & -2.67 & 2.68 & $\mathrm{rad} / \mathrm{s}$ \\
\hline & Velocity & -3 & 3 & $\mathrm{rad} / \mathrm{s}$ \\
\hline & Torques & -100 & 100 & $\mathrm{Nm}$ \\
\hline \multirow{3}{*}{ Joint 4} & Position & -2.35 & 2.22 & $\mathrm{rad} / \mathrm{s}$ \\
\hline & Velocity & -3 & 3 & $\mathrm{rad} / \mathrm{s}$ \\
\hline & Torques & -50 & 50 & $\mathrm{Nm}$ \\
\hline \multirow{3}{*}{$\begin{array}{l}\text { End } \\
\text { Effector } \\
\text { Position }\end{array}$} & $\mathrm{x}$ & -1.5 & 1.5 & $\mathrm{~m}$ \\
\hline & $y$ & -0.6 & 2 & $\mathrm{~m}$ \\
\hline & $\alpha$ & -2 & 3 & $\mathrm{rad}$ \\
\hline \multirow{3}{*}{$\begin{array}{l}\text { End } \\
\text { Effector } \\
\text { Velocities }\end{array}$} & $\mathbf{x}$ & -3 & 3 & $\mathrm{~m} / \mathrm{s}$ \\
\hline & $\mathrm{y}$ & -2.5 & 2.5 & $\mathrm{~m} / \mathrm{s}$ \\
\hline & $\alpha$ & -5 & 4.5 & $\mathrm{rad} / \mathrm{s}$ \\
\hline \multirow{3}{*}{$\begin{array}{l}\text { End } \\
\text { Effector } \\
\text { Forces }\end{array}$} & $\mathbf{x}$ & -200 & 200 & $\mathrm{~N}$ \\
\hline & $\mathrm{y}$ & -200 & 200 & $\mathbf{N}$ \\
\hline & $\tau$ & -30 & 30 & $\mathrm{Nm}$ \\
\hline
\end{tabular}




\section{Emergency Stop Buttons}

The emergency stop buttons are wired to the DAQ. A press of the button opens the circuit, disables the motors and releases the magnetic latch.

\section{Acrylic Shield}

In order to prevent the patient from pinching one's hand or other body parts in the manipulator during operation, an acrylic shield designed to be installed alongside the patient

\section{Hall Effect Sensors}

In order to prevent the robot from moving outside of the joint limits established by the software limits, Hall-Effect proximity sensors were installed close to the physical limits of the joints. Activated by the magnetic field of a permanent magnet placed on the other link in question, the sensor sends a signal to the DAQ which prevents any further movement of the joint. The sensors were made by Infineon Technologies and are nonlatching single pole sensors.

\section{Microswitches}

The bar system that activates the mechanical spring release system was designed to include microswitches which function in the same fashion as the emergency stop buttons by disabling the motors and electromagnet through the DAQ.

\subsection{Manufacturing}

The four linkages were manufactured from engineering drawings developed in Pro/Engineer using a manual mill. The four joint stiffener components, after being designed and optimized in Pro/Engineer and Pro/Mechanica, were machined using a Computer Numerically Controlled (CNC) mill. The part files were imported into the manufacturing suite of Pro/Engineer and the tool paths were developed to ensure accurate machining and a smooth surface finish. Figure 4.19 depicts the tool path planning. 
90

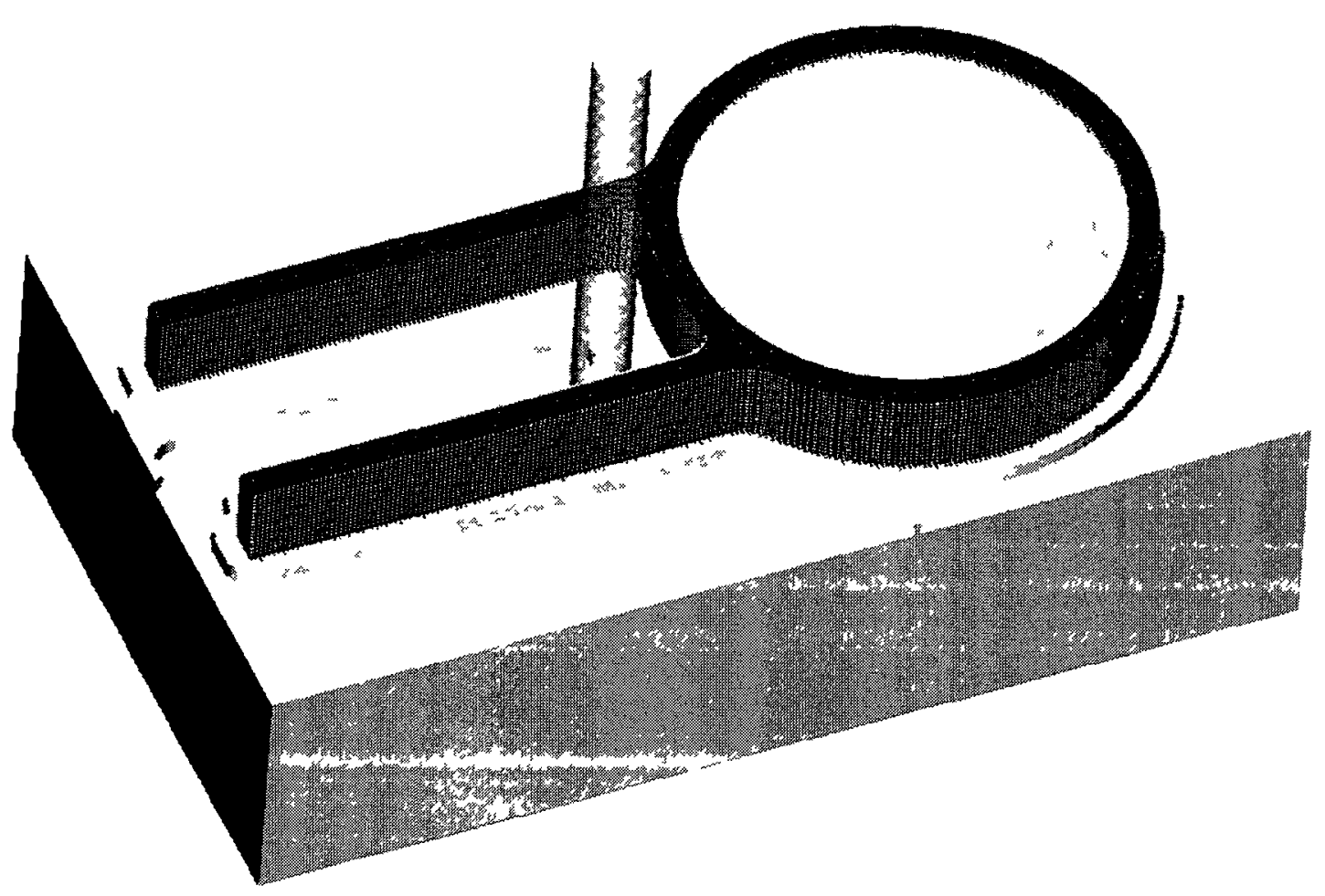

Figure 4.19: Tool Path Creation

4.5 Finite Element Analysis

The data point used for the analysis of the structure is the $54 \%$ point of the gait cycle. This point corresponds to toe off in the gait cycle. High forces are applied at this point in both the $\mathrm{X}$ and $\mathrm{Y}$ directions as in addition to the force to push off the ground and lift the leg, the user is also pushing backwards, creating anterior shear to push the patient forward. The analysis was completed by applying these loads statically to the structure. The fact that this load is in fact highly transient was taken as a worst case loading condition. The manipulator is in an extended configuration at this point in the gait cycle, creating higher internal loads due to the increased length of the moment arm. The finite element analysis achieved a maximum ninth order polynomial fit of the stress data in the p-type Pro/Mechanica analysis and converged within 5\% of all values, the recommended setting. 


\subsubsection{Stress Results}

The Figure 4.20 shows the results of the analysis, depicting the joint configuration and the areas of higher stress. Table 4.8 shows the legend values. The maximum stress on the structure is $467 \mathrm{MPa}$. The peak stress values occur at stress concentrations created at the mating points between components of the joints and may not be representative of the real loading conditions. Figure 4.21 focuses on the second joint showing the area of high stress caused at the interface between the Harmonic Drives and the joint structure.

Table 4.7: Loading Condition for Finite Element Analysis

\begin{tabular}{ll}
\hline Force & Value \\
\hline $\mathrm{F}_{\mathrm{x}}$ & $-1259.2 \mathrm{~N}$ \\
$\mathrm{~F}_{\mathrm{y}}$ & $-157.8 \mathrm{~N}$ \\
$\mathrm{~T}_{\mathrm{xy}}$ & $-12.72 \mathrm{Nm}$ \\
\hline
\end{tabular}

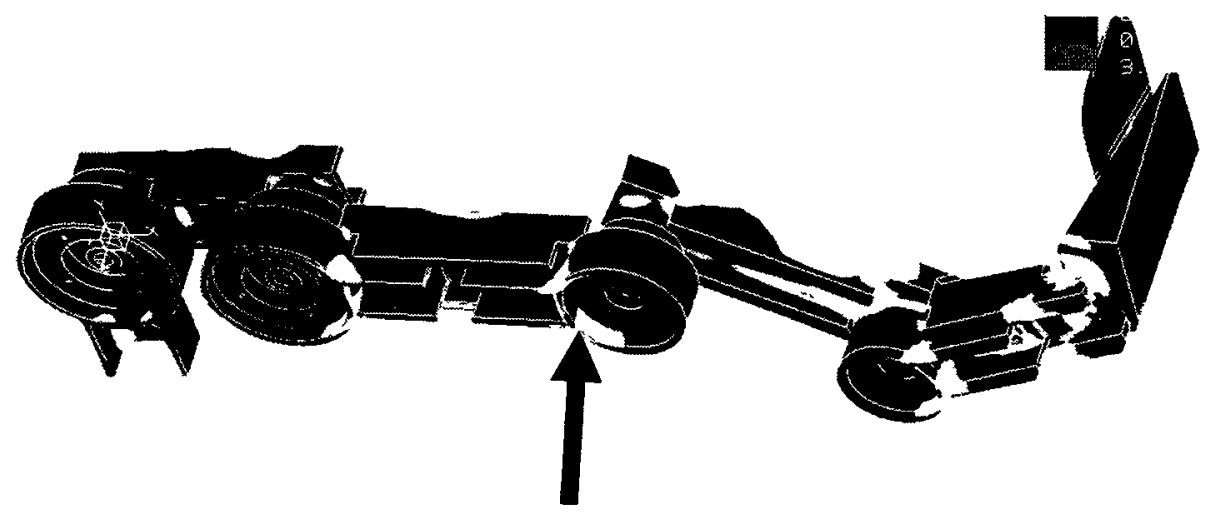

Figure 4.20: Stress Distribution 
Table 4.8: Legend Values

\begin{tabular}{ll}
\hline Colour & Load Range (MPa) \\
\hline Dark & 0 to 21.25 \\
Med & 21.25 to 42.5 \\
Light & 42.5 to 63.75 \\
White & 63.75 and Above \\
\hline
\end{tabular}

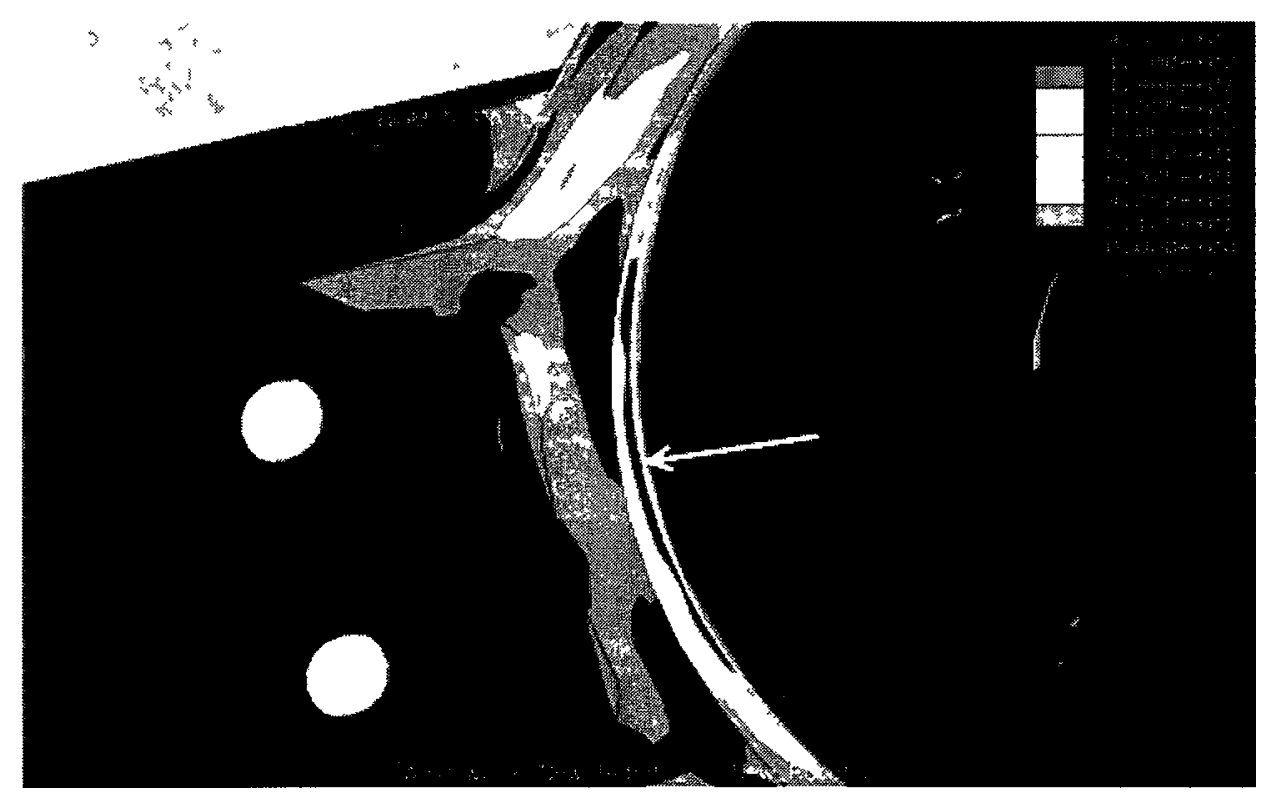

Figure 4.21: Location of Maximum Stress in Joint 2

\subsubsection{Displacement Results}

Figure 4.22 through Figure 4.25 show the deflection results of the FEA analysis. The total magnitude of the deflection is $17.3 \mathrm{~mm}$, with the out of plane, $Z$, direction contributing significantly with $15.3 \mathrm{~mm}$. The design maximum deflection under this worst case scenario was set at $20 \mathrm{~mm}$. Extension of the manipulator under the $1260 \mathrm{~N}$ load is $8.51 \mathrm{~mm}$ while the end effector drops a maximum of $2.48 \mathrm{~mm}$ under the $157.8 \mathrm{~N}$ load. A significant source of this deflection is the joints, though the design significantly reduced stresses and deflections, minimal returns on stiffness were achieved by 
increasing the dimensions of the joint. The simulation overestimates the stiffness of the Harmonic Drive components as they were modeled as solid pieces of mild steel. As the Harmonic Drives are assembled from varıous components, under load they strain against one another and there are some deflections.
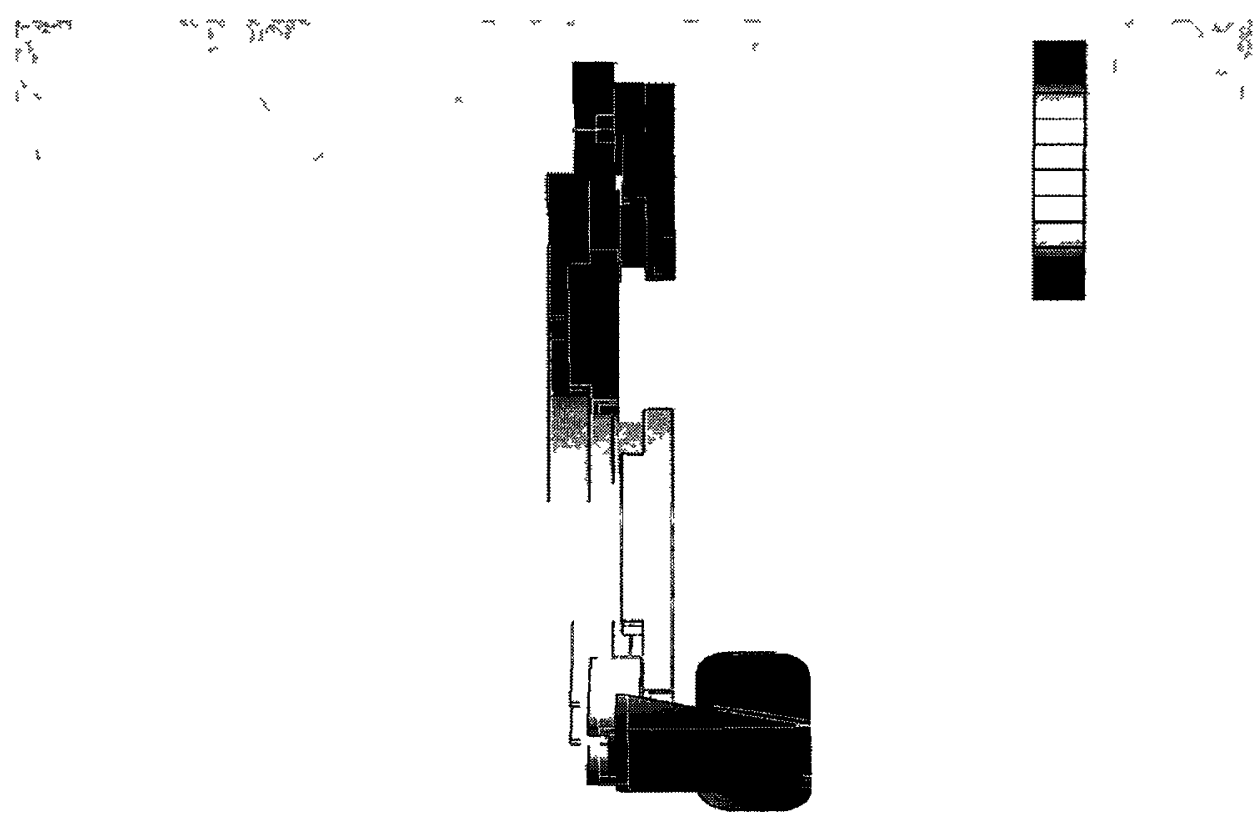

Figure 4.22: Displacement Magnitude 


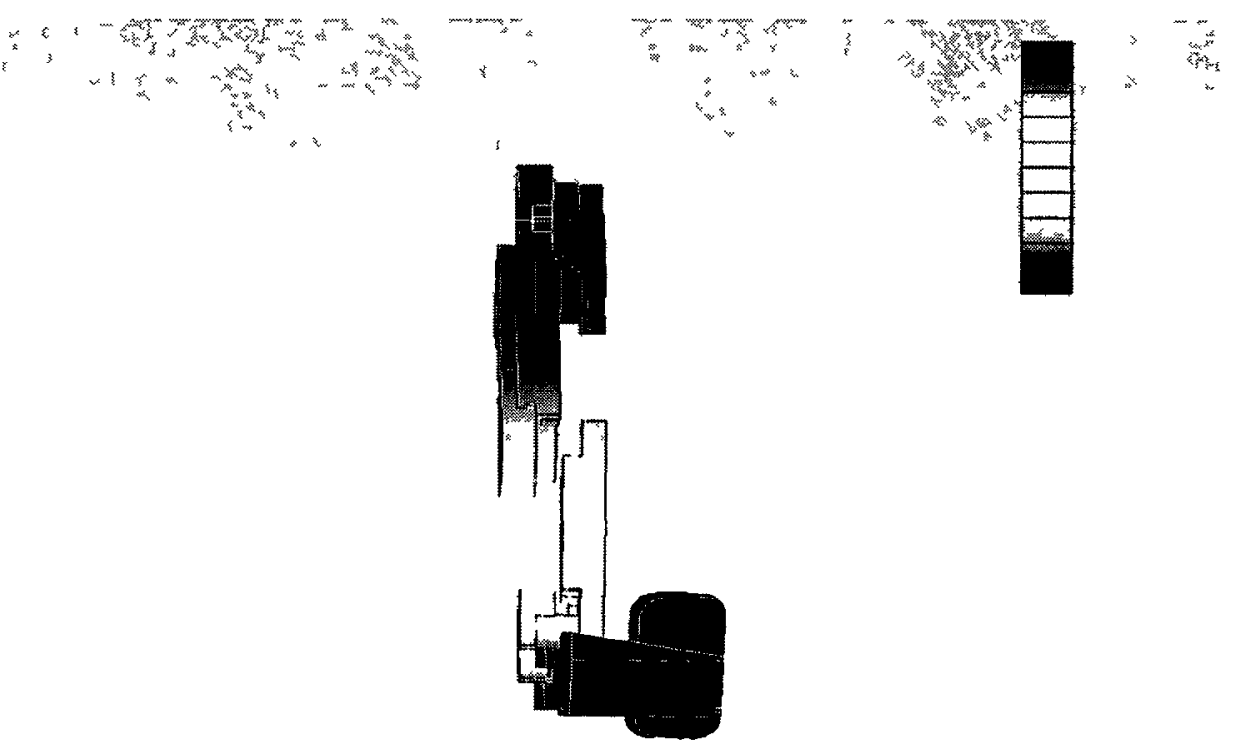

Figure 4.23: Displacement in X Direction

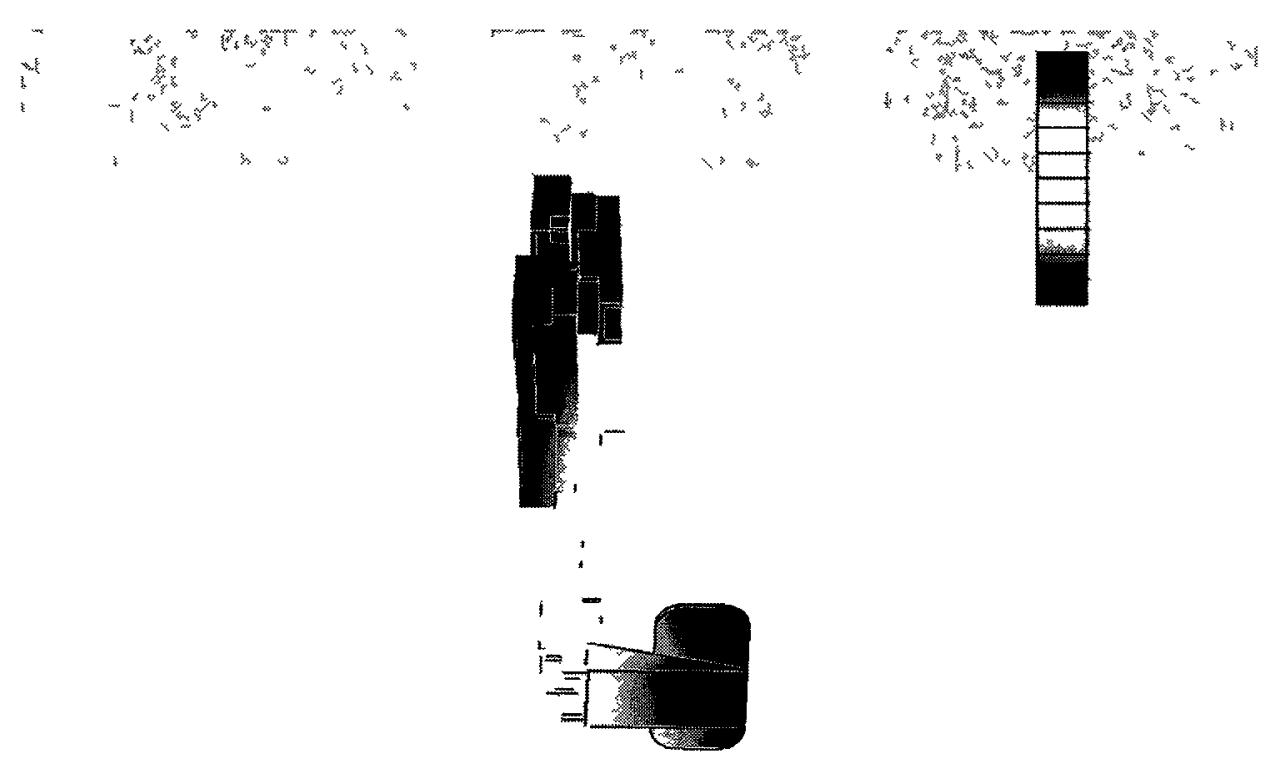

Figure 4.24: Displacement in Y Direction 


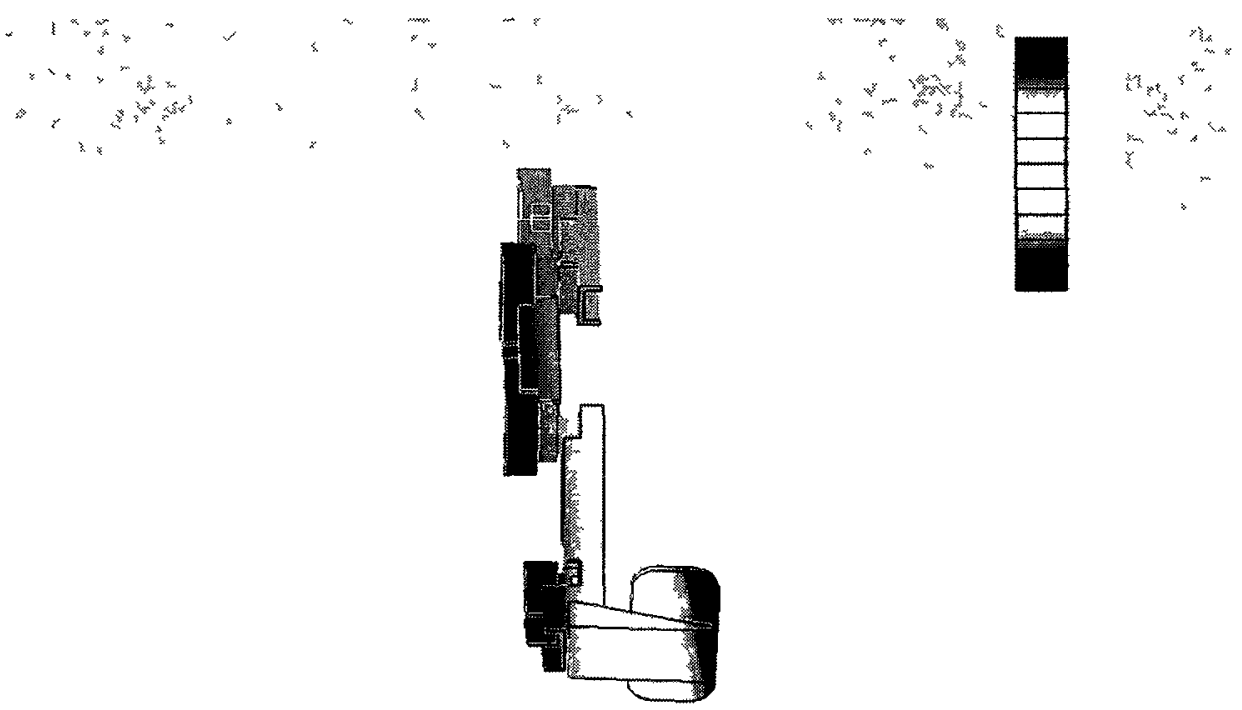

Figure 4.25: Deflection in $\mathbf{Z}$. Direction

Table 4.9: Displacement Results

\begin{tabular}{cc}
\hline & $\begin{array}{c}\text { Maximum } \\
\text { Displacement (mm) }\end{array}$ \\
\hline Extension Displacement (x direction) & 8.51 \\
Vertical Displacement (y direction) & -2.48 \\
Out of Plane Displacement (z direction) & 15.3 \\
Displacement Magnitude (combined) & 17.3 \\
\hline
\end{tabular}

\subsection{Work Envelope}

From the link lengths in Table 3.3 and taking into account the joint limits presented in Table 4.6 a plot of the end effector workspace was developed iterating the joint angles of 
all four joints every five degrees and checking for self collisions. Figure 4.26 shows the results of this analysis, including the trajectories of the male and female patient outlined in 3.4.1 of the design requirements. The limit in the workspace is due to collisions with the current base, which is a modified office desk. An updated platform would allow the manipulator to cover the entire range of the patient's leg.

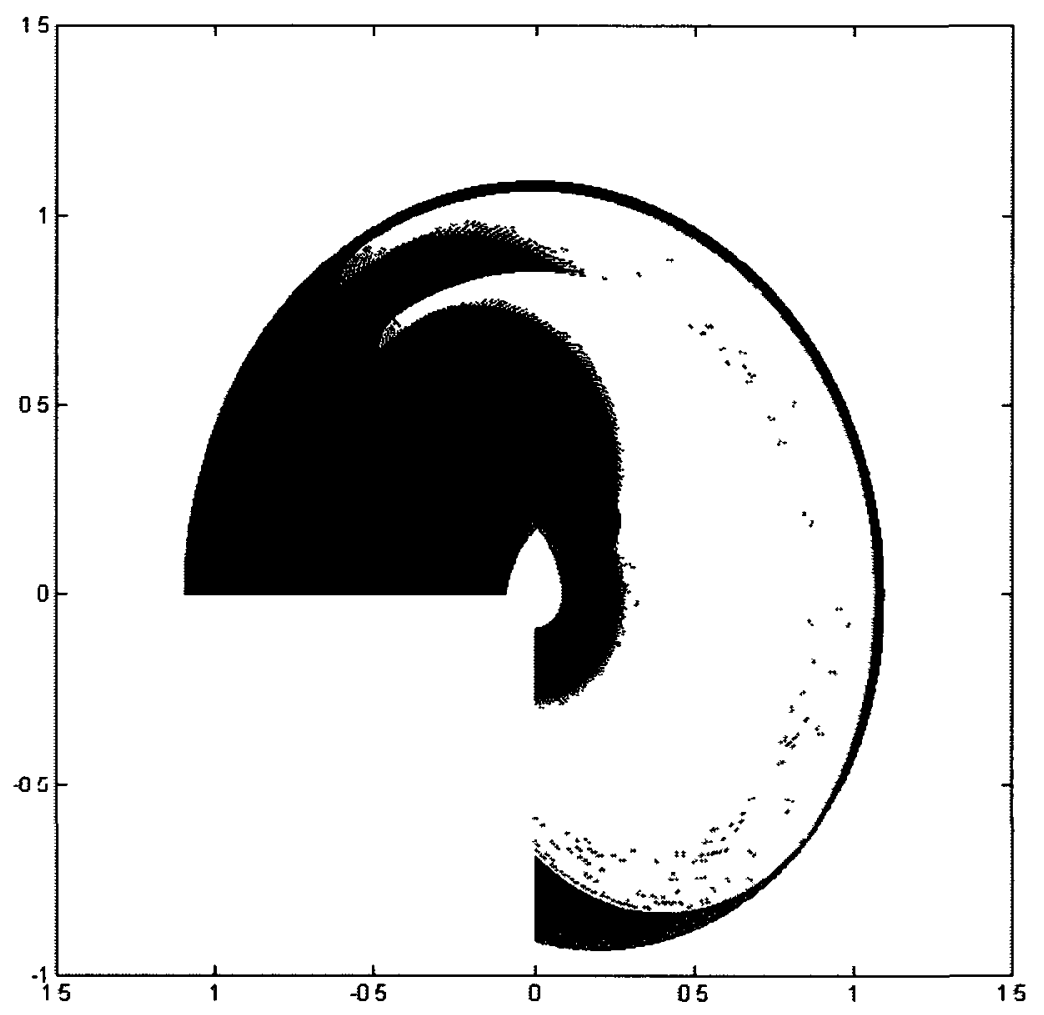

Figure 4.26: Workspace of Manjpulator, Neglecting Collisions with Table

\subsection{Final Design}

Figure 4.27 details the final CAD layout of the manipulator. Item 1 is the Danaher AKM series motor that is coupled with the Harmonic Drive CSG gearbox. These are actuated by the Danaher S200 Drive unit, not shown. The four I-beam links are represented by 
Item 2. Item 3 is the joint design that mates the actuators to the joint. A stiffening ring was designed to minimize the end effector deflection due to joint compliance. In order to space the joints sufficiently so that a large range of motion is available, inserts were machined to fit at the output face of the Harmonic Drive. Item 5 is the end effector, which is mated with a foot attachment plate without safety features installed. Figure 4.28 shows the design from multiple angles.

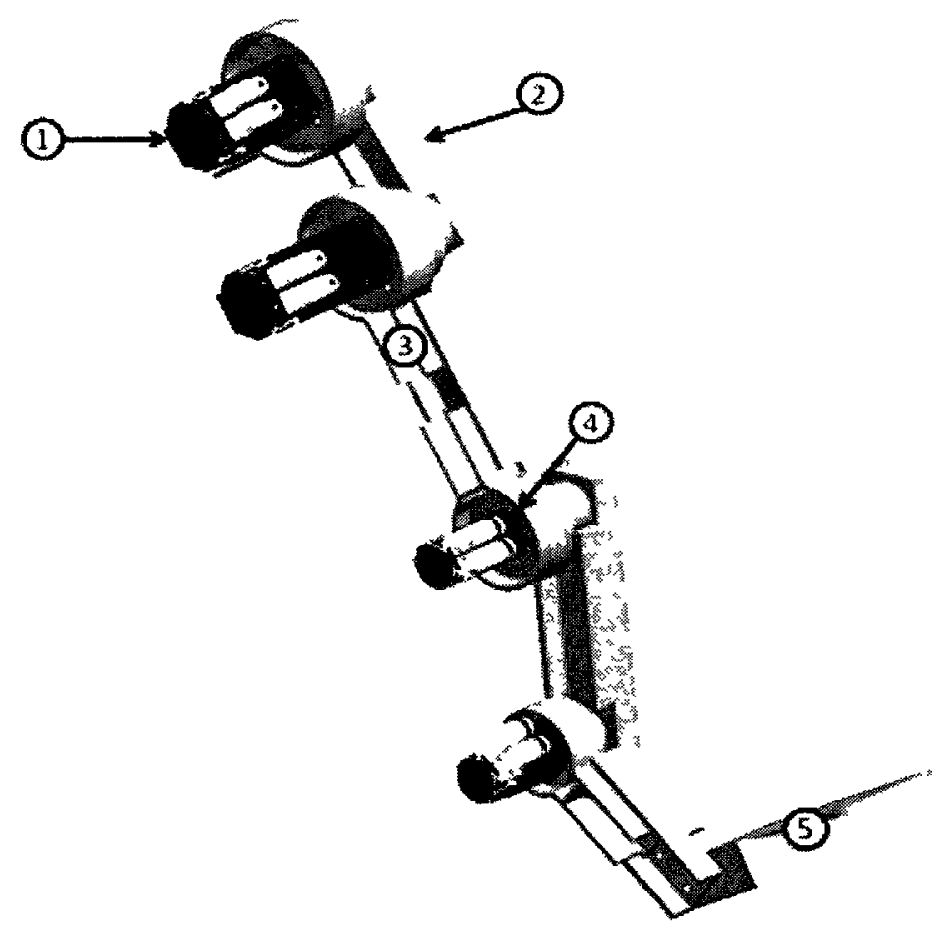

Figure 4.27: Final CAD Design of Structure 


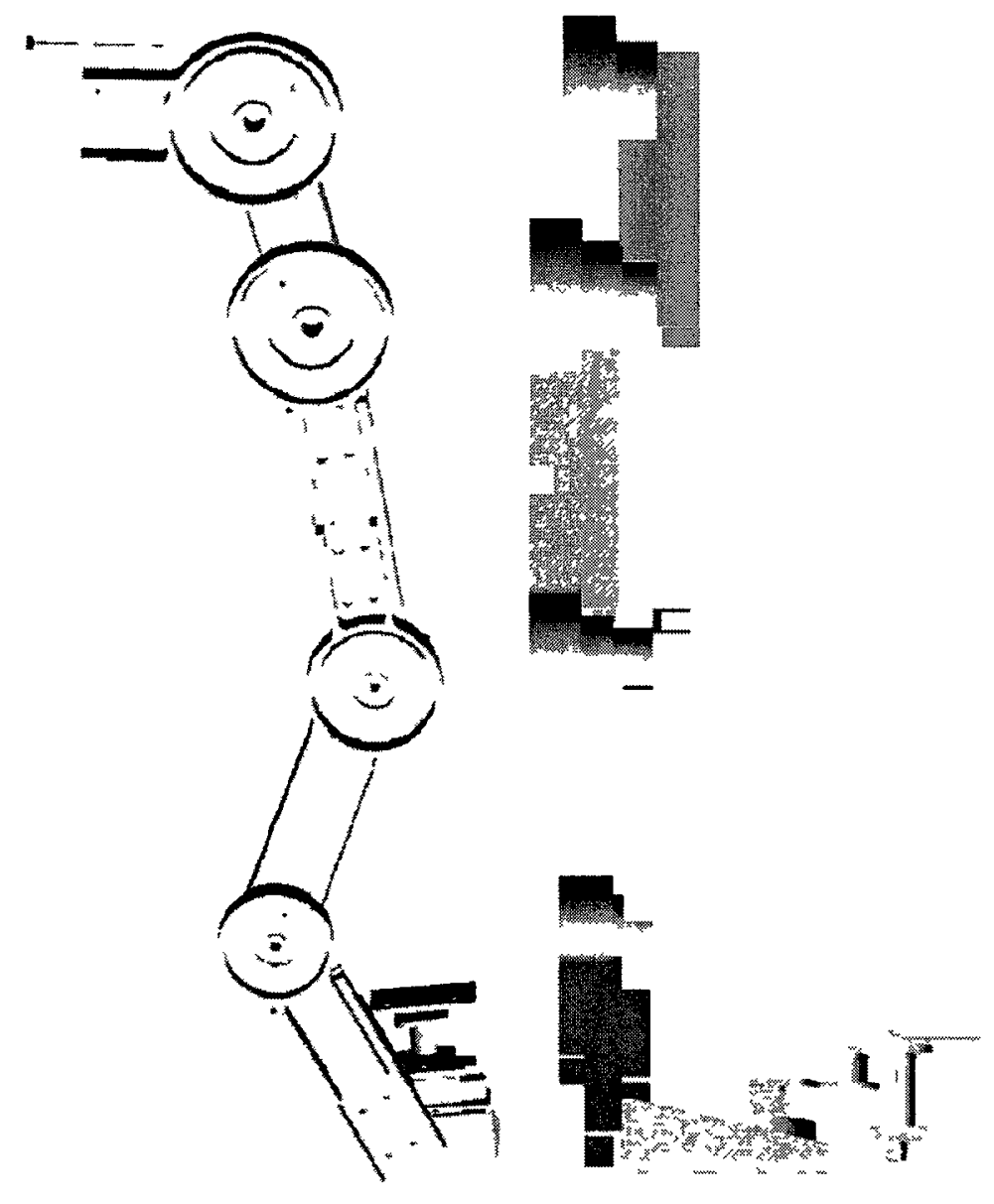

Figure 4.28: Images of Design of Manipulator

\subsection{Summary}

This chapter presented the detailed design and development of the ViGRR manipulator including the design of the structure, link and joints to meet the design requirements of the system. The design was analyzed using the Pro/Mechanica finite element suite and manufactured in house in the Mechanical and Aerospace Department Machine Shop. Chapter 5 presents the results of the calibration of the system as well as detailing the experiments that were performed to verify the design and various controllers. 


\section{Chapter 5: Calibration and Experimentation}

Chapters 3 and 4 detailed the design and analysis of the ViGRR manipulator. Along with the controllers developed in [45] the robotic platform was prepared for initial tests. In order to ensure that ViGRR is operating correctly and can track desired motion or deliver the expected forces, the manipulator had to be calibrated and the parameters identified and verified. In the process of integration, each link was individually tested to estimate its dynamic parameters. The robot was then assembled, and using the redundant degree of freedom the manipulator was kinematically calibrated. The final parameter identification was performed for all four degrees of freedom applying sinusoidal inputs in joint space control.

The remainder of the chapter covers the experiments that were performed on the robot to verify the controller. A joint level control was performed to determine the accuracy of the joint angle control. Then, using the redundancy resolution of the manipulator, a task space circular trajectory was run. Lastly, to test the force feedback control system an admittance controller was implemented with user feedback on a computer screen where the intent was for the user to follow a trajectory tracing a circular pattern at a specific rate.

\subsection{Overview of ViGRR Controller}

In parallel to the design and manufacture of the manipulator, a custom controller was developed for the platform in [45]. Section 5.1 presents the control modes and necessary information to understand the remaining cooperative work. The control scheme has an inner and outer loop to the model. The inner loop takes into account the nonlinear 
dynamics of the manipulator while the outer loop is devoted to tracking, either in joint or task space. Figure 5.1 shows the inner and outer loop control within the system. The trajectory generator is given either a joint level command or a task space command. From this information the trajectory generator plans the required joint space signals for the motors to articulate the joints to achieve this motion. This information is fed to the tracking controller that compares the desired trajectory from the trajectory generator to the outputs from the encoders on the motors and the force torque sensor to determine the error in the system and takes action to correct this error. This information is fed through a controller that compensated for the nonlinear dynamics of the model. This command is then output to the physical system where the force torque sensor and encoders record the results of the input to the system and this information is sent back to the trajectory tracking controller.

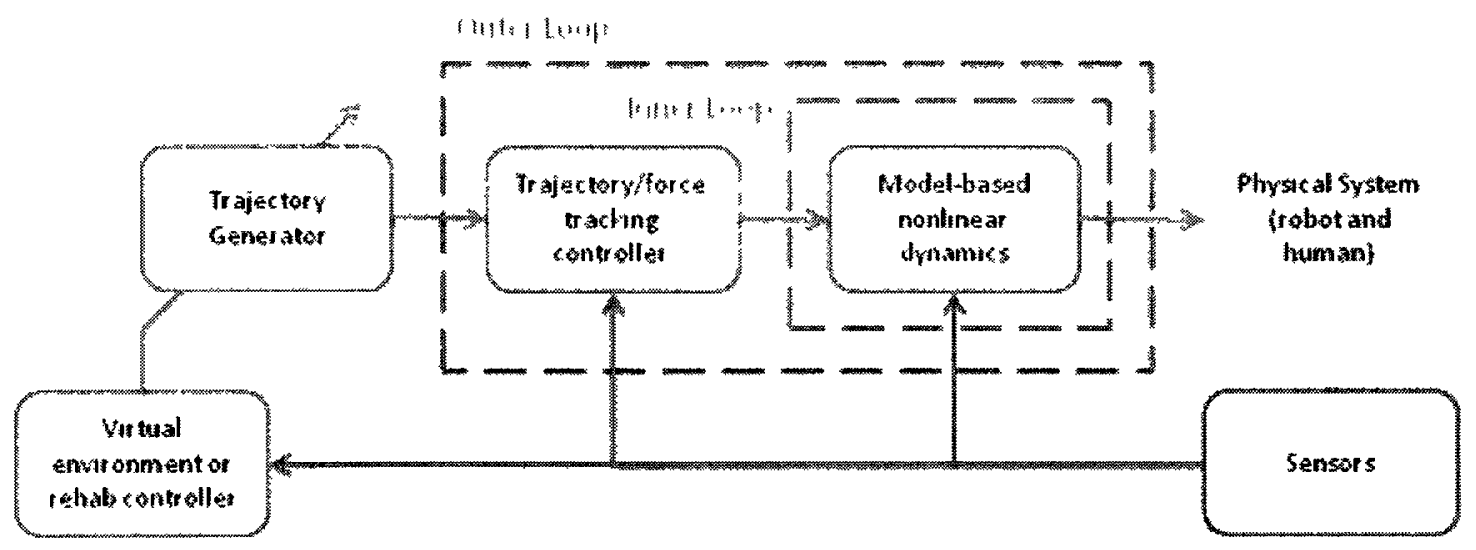

Figure 5.1: Control Scheme for ViGRR

\subsubsection{Trajectory Generation and Position Control}

The trajectory generators are designed in both joint space $q \in R^{4}$ and task space $X \in R^{3}$. In joint space, the path between current position and desired location is fit with a $5^{\text {th }}$ order position polynomial to allow for a smooth, continuous velocity and acceleration profile upon differentiation. In the case of a continuous motion, there is a five second smoothing 
time at the beginning of the trajectory to allow the manipulator to go from a fixed position to the trajectory desired.

In cartesian space, the trajectory generator operates in a similar manner taking the three degrees of freedom end effector trajectory and through a redundancy resolution scheme of the inverse kinematics, determines the joint level trajectories to achieve the end effector motion.

\subsubsection{Admittance Controller}

The derivation of the admittance controller as a variation of the impedance controller is presented in [45]. The admittance controller takes the applied load through the Force Torque sensor and through the inverse dynamics model of the manipulator determines the desired motion of the end effector taking into account virtual inertias, dampings and stiffnesses of the system.

\subsection{Single Degree of Freedom Parameter Identification}

Prior to assembling the platform, an initial set of parameters for the links needed to be determined. Link $n-1$ was clamped to a flat surface with the components of link $n$ attached. Joint $\mathrm{n}$ was with a varying sinusoidal input, with changing amplitudes, frequencies and offset angles to excite multiple dynamic modes while recording the applied torques and angular position of the joints. The angular velocity and acceleration were determined by differentiating the filtered position data. The dynamics equation used to describe the motion of the calibration, from [45] is

$$
\tau_{r_{k}}=\left(I_{k}+I_{e f f k}\right) \ddot{q}_{k}+B_{e f k} \dot{q}_{k}+v_{k} \tanh \left(u_{k} \dot{q}_{k}\right)+m_{k} l_{c k} g \sin \left(q_{k}\right)
$$

Isolating the coefficients gives the form

$$
\tau_{r_{k}}=D\left(q_{k}, \dot{q}_{k}, \ddot{q}_{k}\right) \Theta=\left[\begin{array}{llll}
\ddot{q}_{k} & \dot{q}_{k} & \tanh \left(u_{k} \dot{q}_{k}\right) & \sin \left(q_{k}\right)
\end{array}\right] \Theta
$$

where 


$$
\Theta=\left\{\begin{array}{c}
I_{k}+I_{e f f k} \\
B_{e f f k} \\
v_{k} \\
m_{k} l_{C k} g
\end{array}\right\}
$$

Across $n$ data points, 5.3, from the trajectory a least squares fit, 5.4, of the 4 by $N$ matrix determines the updated parameters.

$$
\begin{gathered}
T_{r_{k}}=D \Theta \\
\left\{\begin{array}{c}
\left(\tau_{\text {rk }}\right)_{1} \\
\left(\tau_{\text {rk }}\right)_{2} \\
\vdots \\
\left(\tau_{\text {rk }}\right)_{N}
\end{array}\right\}=\left[\begin{array}{c}
D\left(q_{k}, \dot{q}_{k}, \ddot{q}_{k}\right)_{1} \\
D\left(q_{k}, \dot{q}_{k}, \ddot{q}_{k}\right)_{2} \\
\vdots \\
D\left(q_{k}, \dot{q}_{k}, \ddot{q}_{k}\right)_{N}
\end{array}\right] \Theta \\
\hat{\Theta}=\left(D^{T} D\right)^{-1}\left(D^{T} T_{r k}\right)
\end{gathered}
$$

The parameters are dependent on the value of the coulomb friction, $u_{k}$, from 5.2. As the coulomb friction is an unknown value, an iterative optimization of the least squares fit was performed. The parameter $u_{k}$ was determined by iterating the parameter between 0.1 and 20 with a step size of 0.01 to find the lowest least squares value of the other parameters. Weighing the links to determine the mass $m_{k}$, and using the CAD files to determine an estimate of the inertia $I_{k}$ and solving for $I_{\text {eff } k}$ by $I_{\text {eff } k}=\hat{\Theta}-I_{k}$ the individual link parameters were all determined and are presented in Table 5.1.

The applied torques and the re-calculated torques from the model were plotted in Figure 5.2 through Figure 5.5. Subtracting the measured torques from the modeled values yields the error plots, Figure 5.6 through Figure 5.9.

The errors in the torque values are increased at the peaks of the absolute torque values. A closer inspection of the peak torque areas, in Figure 5.10 through Figure 5.13 of the four actuator data shows noisy torque signals in the model values and show significant additional fluctuation in the measured torque values.

Joints 1 and 2 appear to have persisting fluctuations in the measured data compared to the model, but at a much lower frequency. There does not appear to be a significant tracking error on these actuators as there is no significant error band in the data shown. 
The actuators used for joints 1 and 2 have a significantly higher load capacity than the other two motors. The increased error in the torque data for these joints compared to joint 3 may just be an artifact of this.

Joints 3 and 4, Figure 5.12 and Figure 5.13, though under similar load conditions respond very differently. With joint 3 , though having fluctuation patterns similar to joints 1 and 2, the measured torques do not accurately follow the model and consistently applied insufficient torques to the motor. As these variations are in the command torques this is not a by-product of the calibration or the dynamic model and though there are additional fluctuations the command torque does track these model variations. Joint 4 has a very regular sinusoid shape torque signal for both the model and the measured torques, tracking the altering directions of the model torque accurately, though significantly overshooting and having to compensate on the next cycle. A possible source of this error is mechanical, a misalignment in the Harmonic Drive or between the motor and the Harmonic Drive could create a situation where there is a very high static friction that requires a high torque to overcome. Alternatively the error may be caused by an issue with the PD controller. In any case the final motion was smooth and the parameters were deemed usable with low gains for the four degrees of freedom calibration presented in Section 5.3. Though there is some high frequency variations in the torque data, the errors are not significant given the capacities of the actuators. 


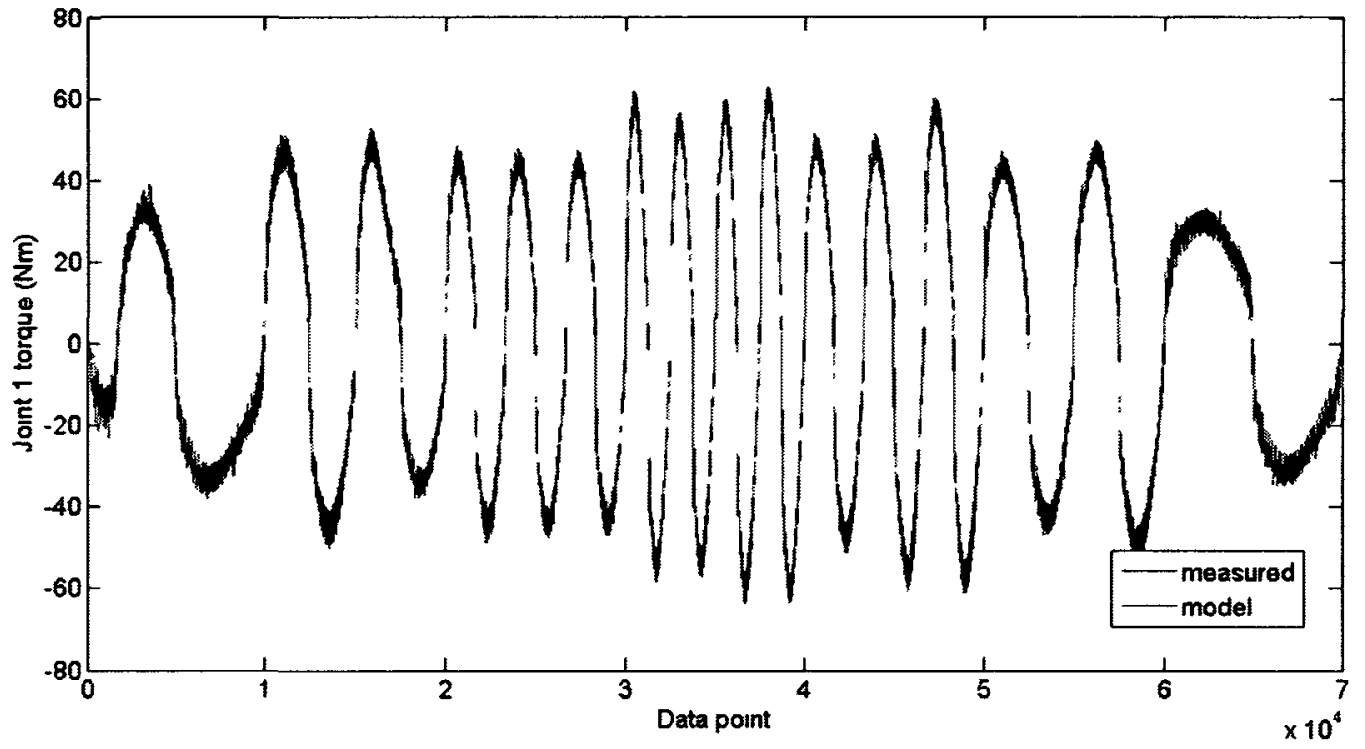

Figure 5.2: Measured and Modeled Joint 1 Torques - Single DoF Calibration

Table 5.1: Results of Calibration

\begin{tabular}{cc}
\hline Parameter & Calibrated Value \\
\hline $\mathrm{l}_{\mathrm{c} 1}$ & 0.2633 \\
$\mathrm{l}_{\mathrm{c} 2}$ & 0.1051 \\
$\mathrm{l}_{\mathrm{c} 3}$ & 0.1961 \\
$\mathrm{l}_{\mathrm{c} 4}$ & 0.1325 \\
$\mathrm{~m}_{1}$ & 1.1662 \\
$\mathrm{~m}_{2}$ & 13.5075 \\
$\mathrm{~m}_{3}$ & 1.8043 \\
$\mathrm{~m}_{4}$ & 5.7489 \\
$\mathrm{I}_{1}$ & 0.007879 \\
$\mathrm{I}_{2}$ & 0.3992 \\
$\mathrm{I}_{3}$ & 0.02094 \\
$\mathrm{I}_{4}$ & 0.07775 \\
$\mathrm{I}_{\mathrm{eff} 1}$ & 4.2196 \\
$\mathrm{I}_{\mathrm{eff} 2}$ & 4.5830 \\
$\mathrm{I}_{\mathrm{eff} 3}$ & 1.5672
\end{tabular}




$\begin{array}{cc}\mathrm{I}_{\text {eff }} & 0.2675 \\ \mathrm{~B}_{\mathrm{eff1}} & 27.2051 \\ \mathrm{~B}_{\text {eff2 }} & 24.8834 \\ \mathrm{~B}_{\text {eff3 }} & 18.0541 \\ \mathrm{~B}_{\text {eff4 }} & 6.1571 \\ \mathrm{v}_{1} & 15.6736 \\ \mathrm{v}_{2} & 12.8680 \\ \mathrm{v}_{3} & 10.6000 \\ \mathrm{v}_{4} & 4.9839 \\ \mathrm{u}_{1} & 11.70 \\ \mathrm{u}_{2} & 17.11 \\ \mathrm{u}_{3} & 11.72 \\ \mathrm{u}_{4} & 12.23\end{array}$

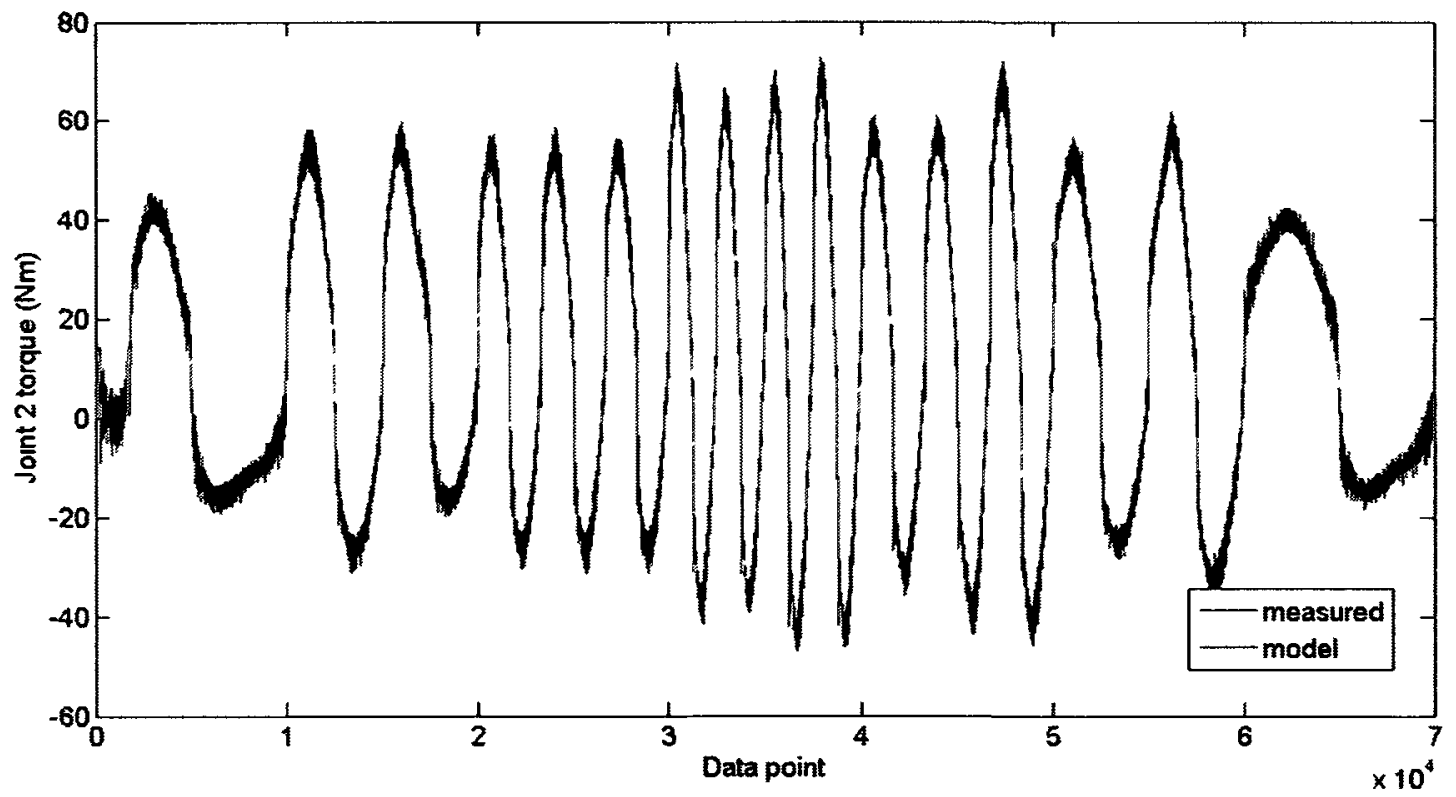

Figure 5.3: Measured and Modeled Joint 2 Torques - Single DoF Calibration 


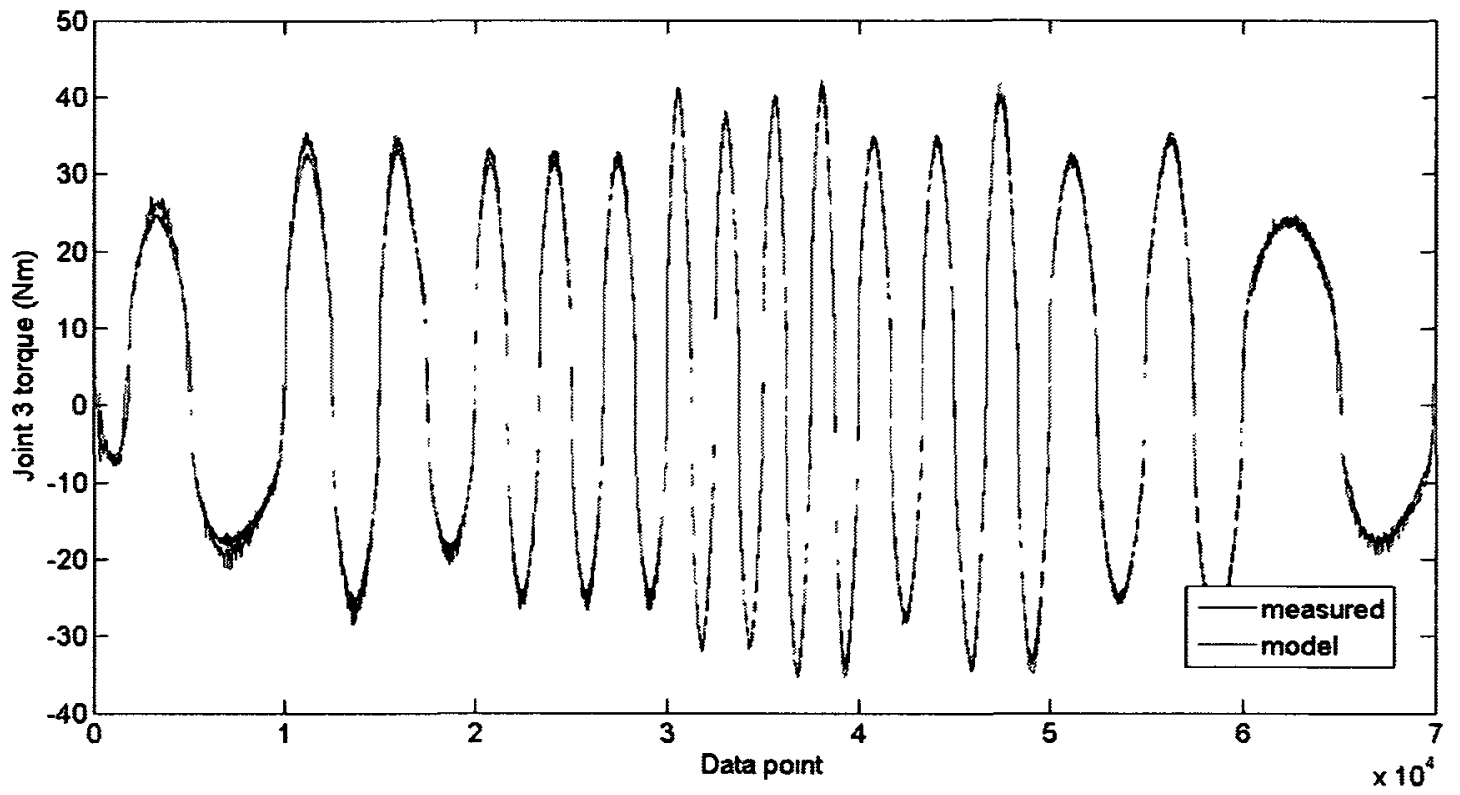

Figure 5.4: Measured and Modeled Joint 3 Torques - Single DoF Calibration

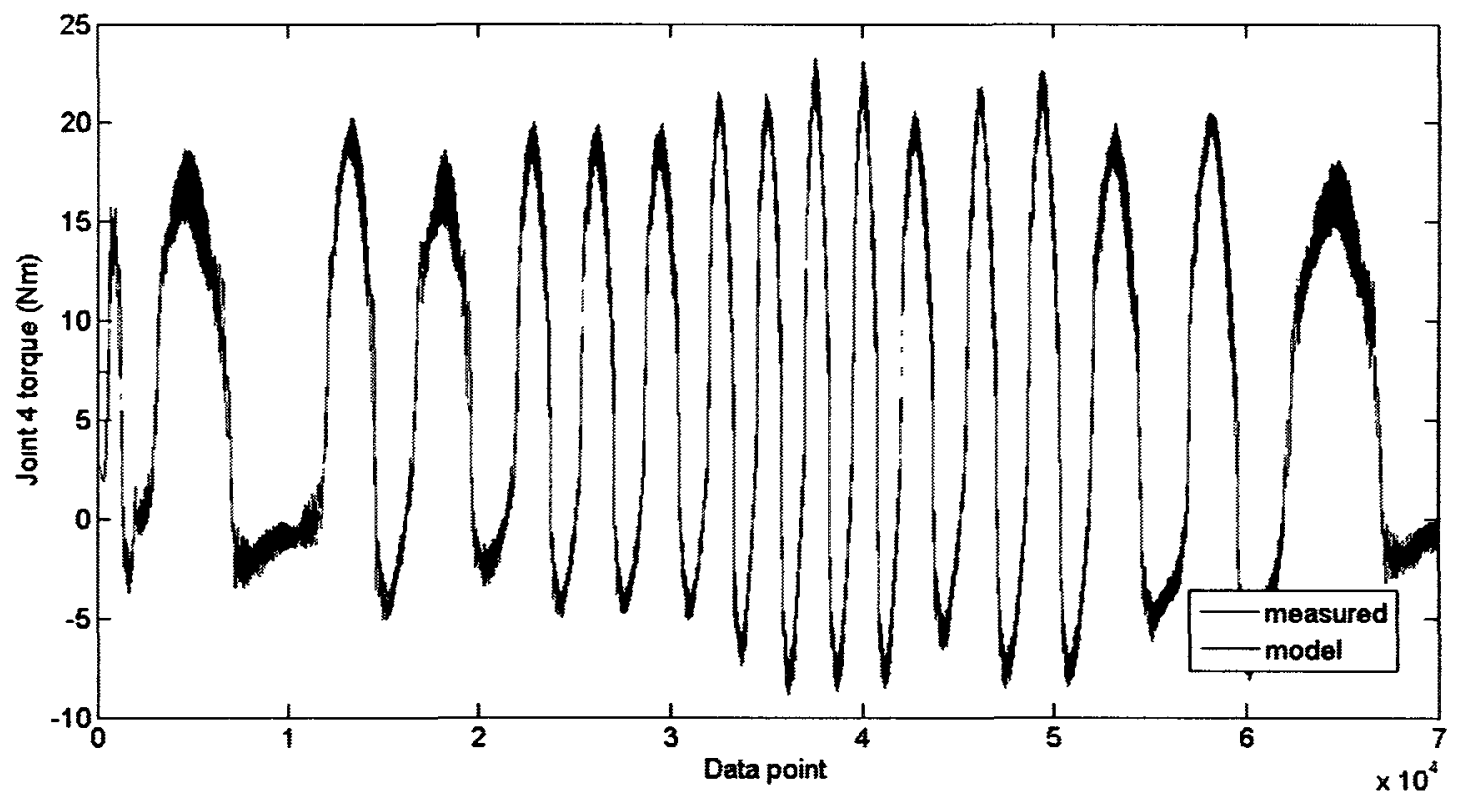

Figure 5.5: Measured and Modeled Joint 4 Torques - Single DoF Calibration 


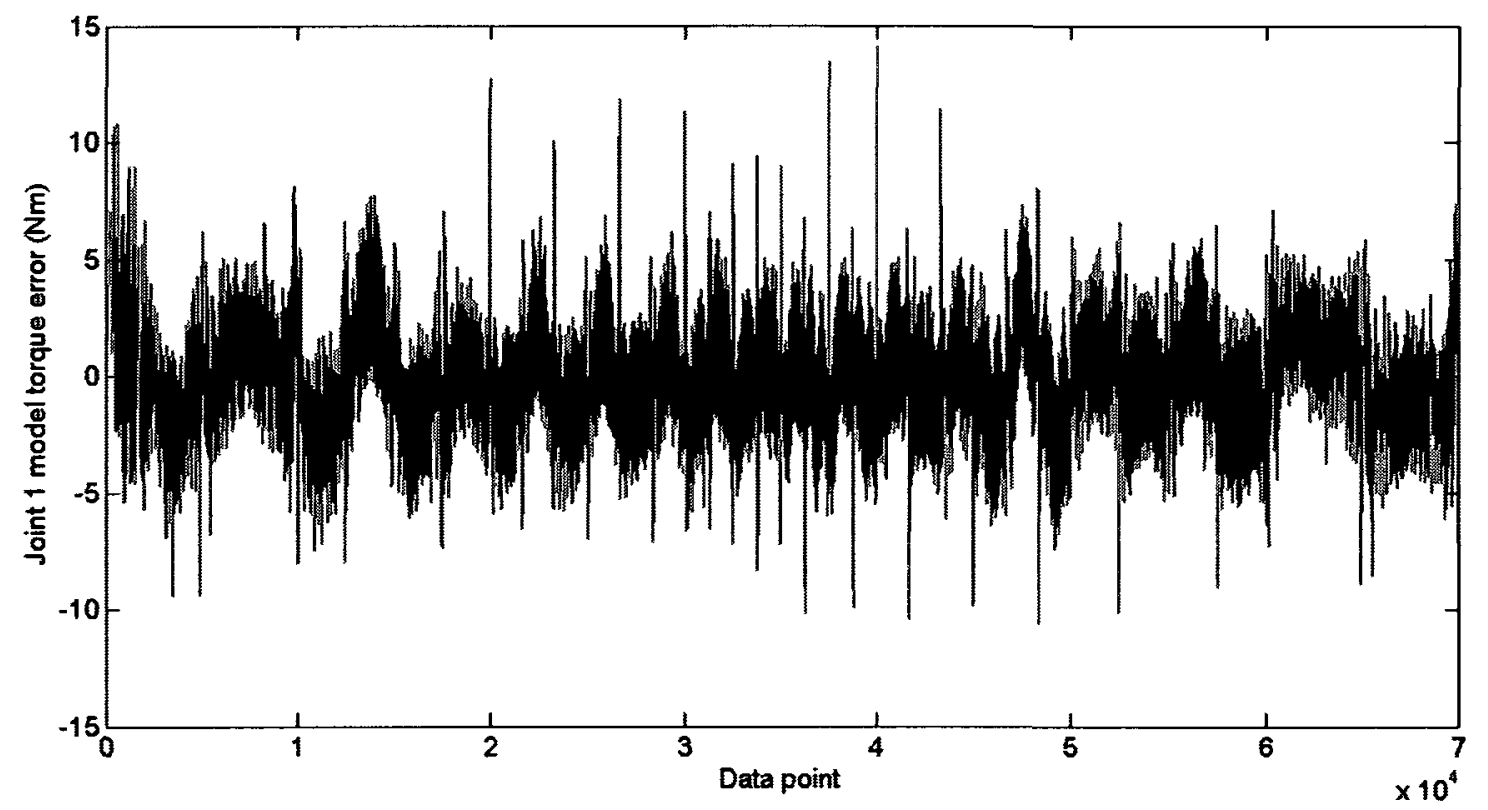

Figure 5.6: Error in Joint 1 Torques - Single DoF Calibration

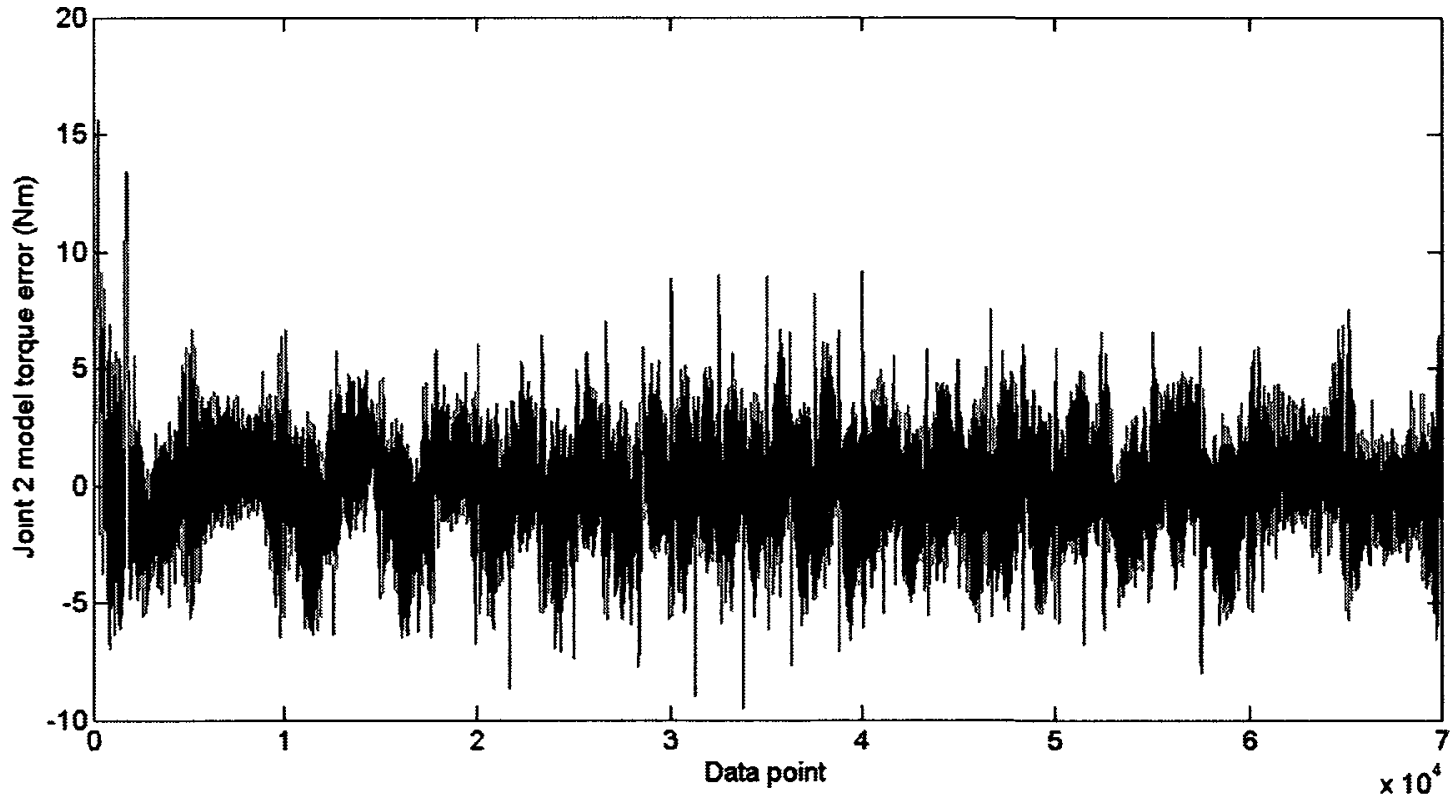

Figure 5.7: Error in Joint 2 Torques - Single DoF Calibration 


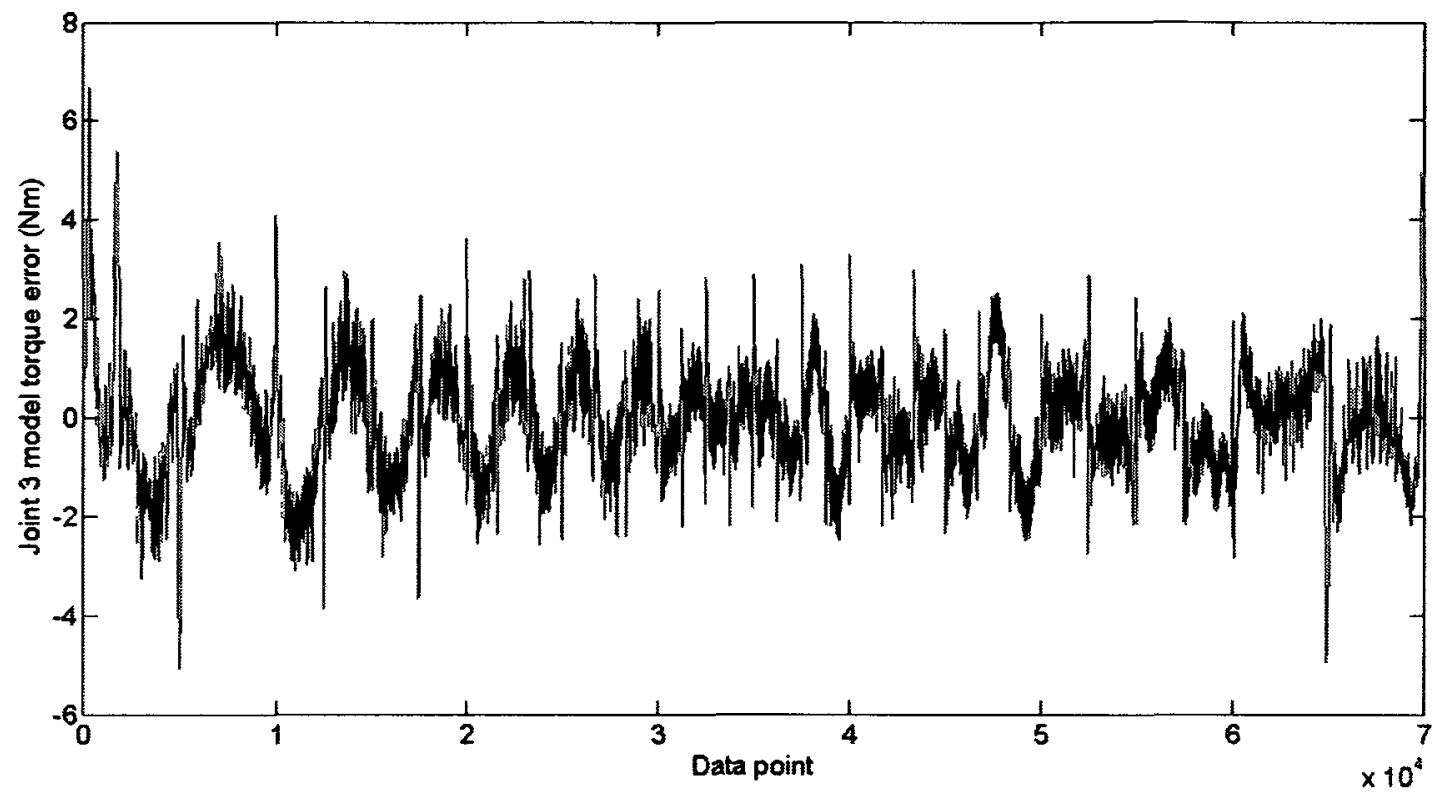

Figure 5.8: Error in Joint 3 Torques - Single DoF Calibration

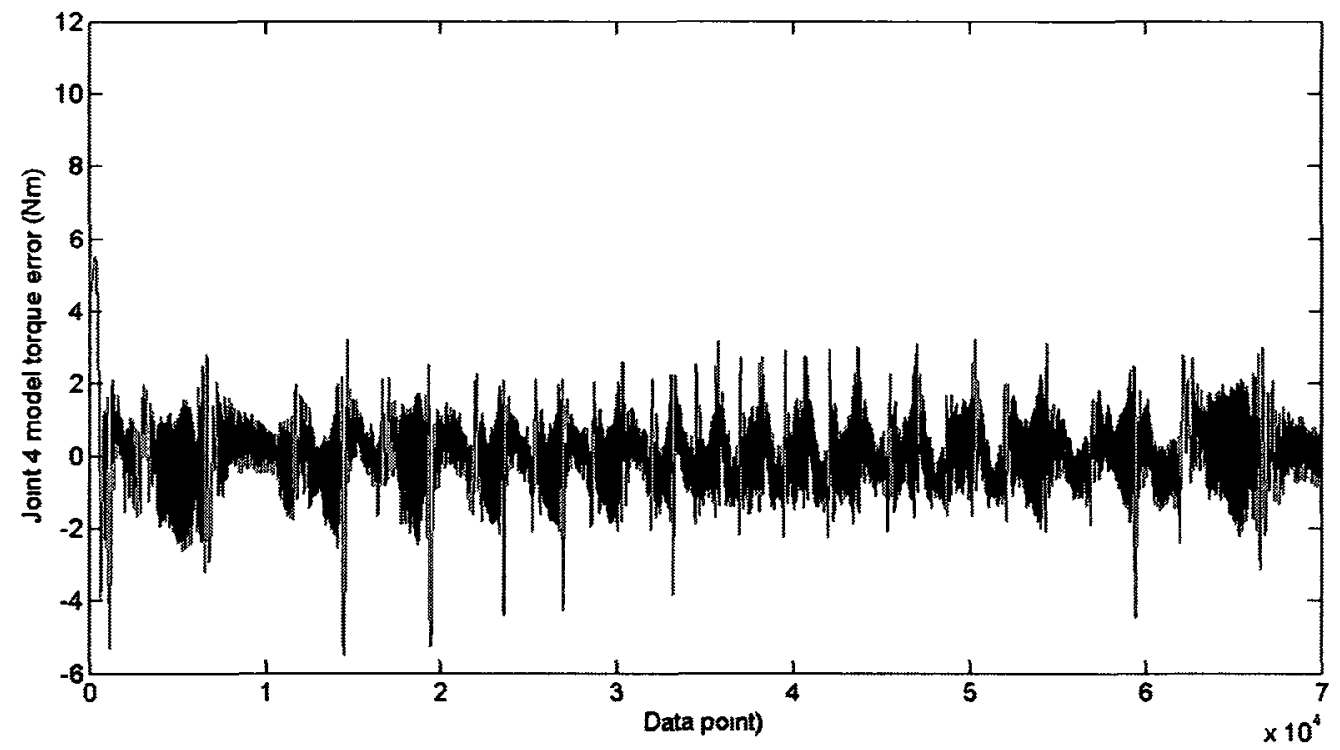

Figure 5.9: Error in Joint 4 Torques - Single DoF Calibration 


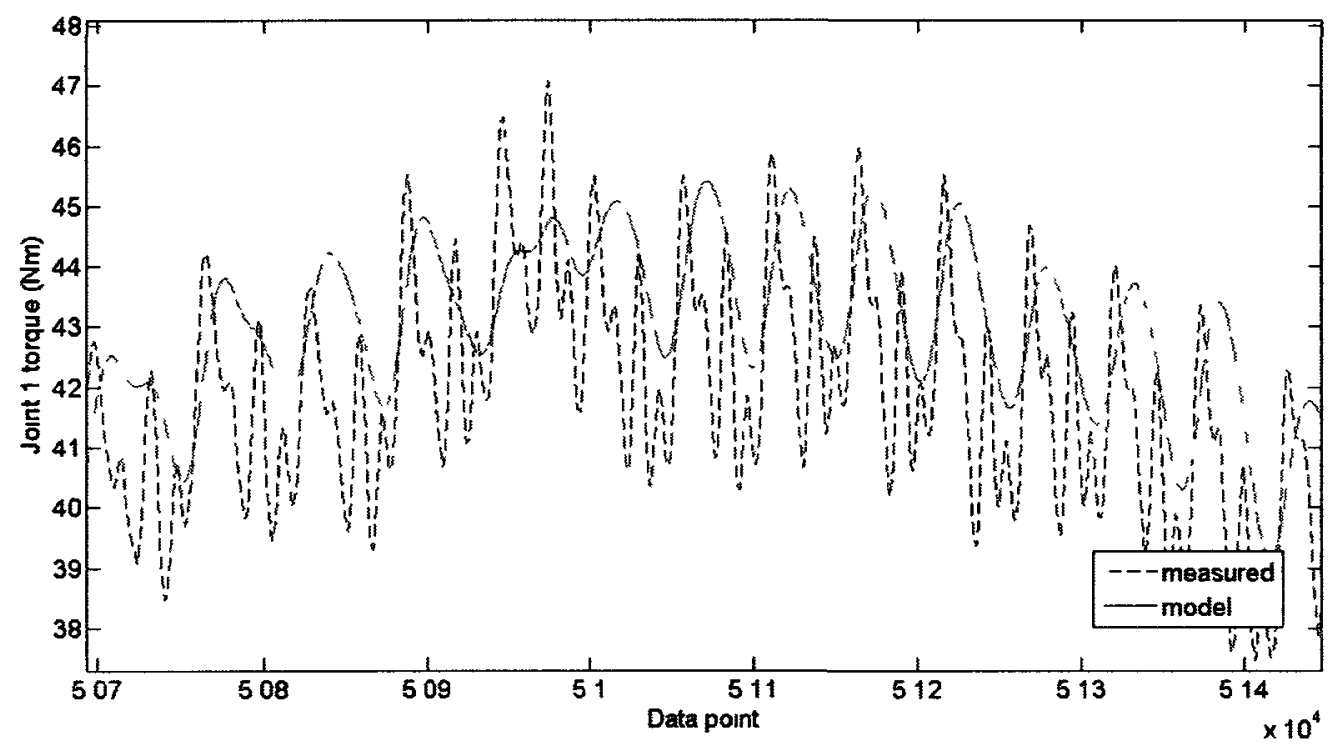

Figure 5.10: Close up of Joint 1 Torque Data

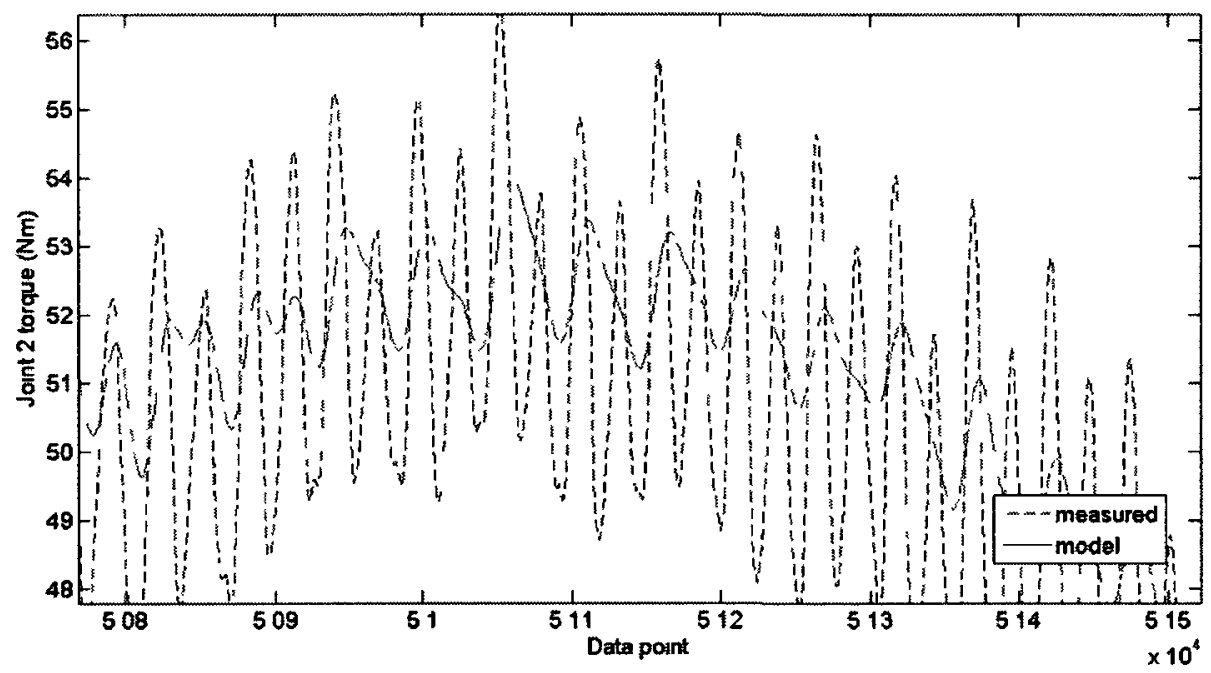

Figure 5.11: Close up of Joint 2 Torque Data 


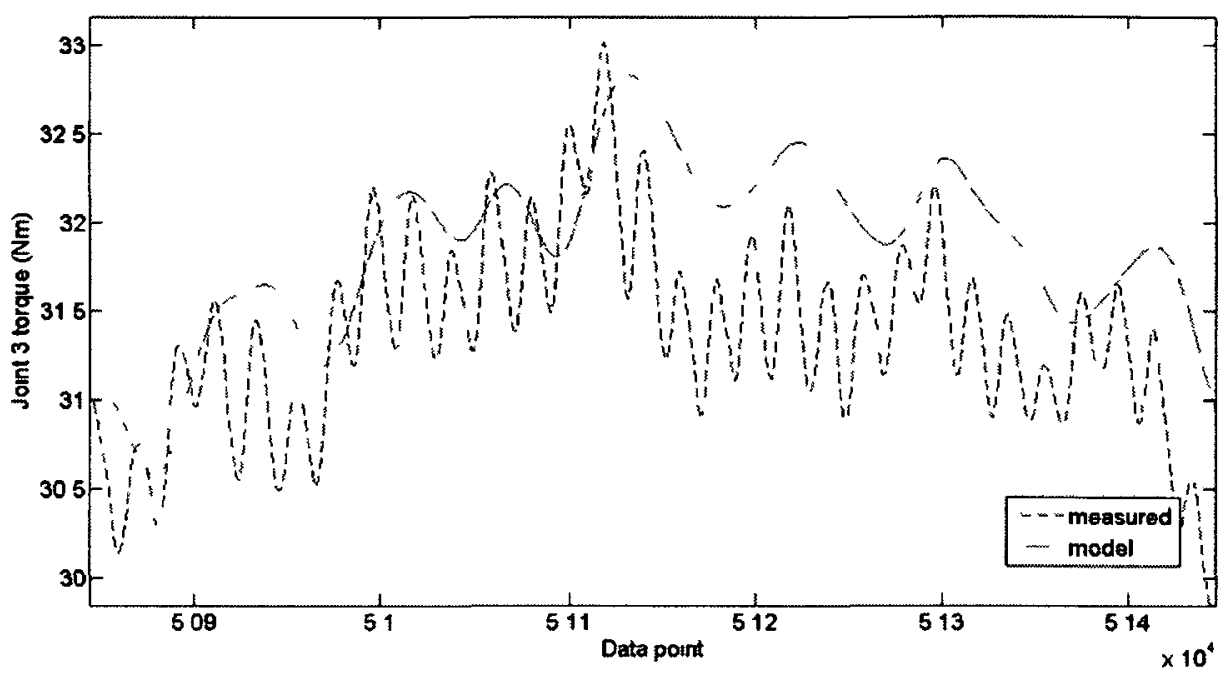

Figure 5.12: Close up of Joint 3 Torque Data

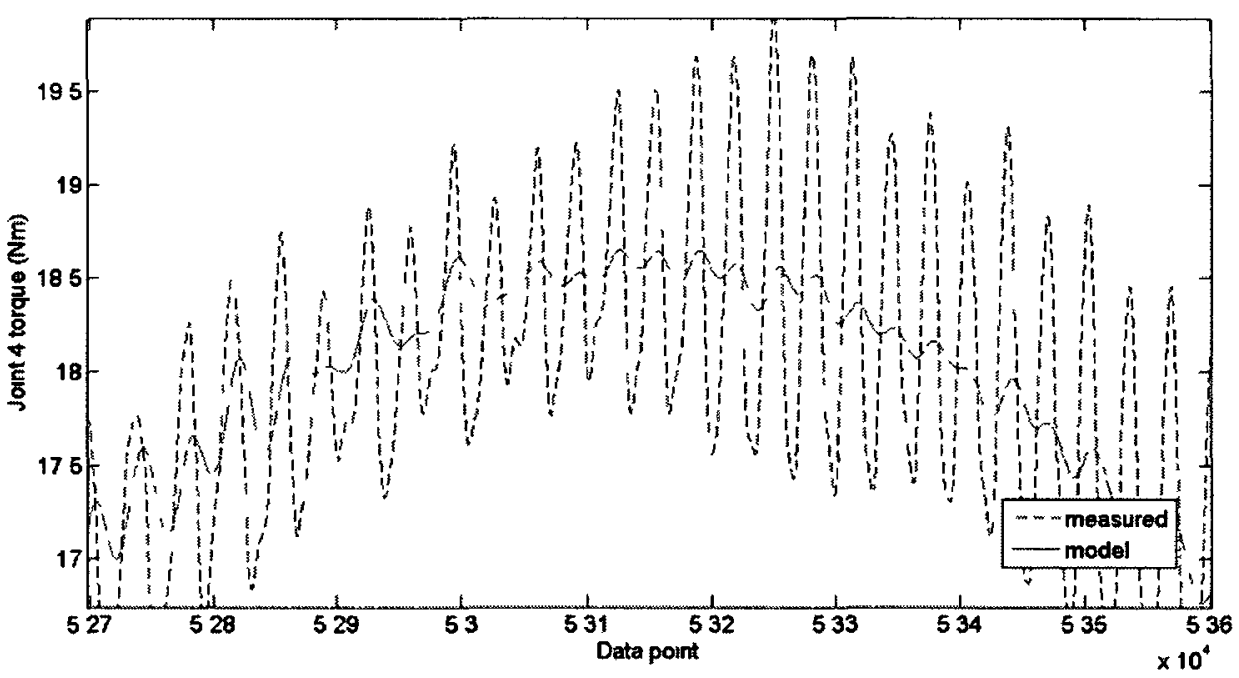

Figure 5.13: Close up of Joint 4 Torque Data 


\subsection{Kinematic Calibration}

The kinematic calibration was performed by rigidly attaching the end effector of the robot to the base platform at a known distance from the base of the manipulator, Figure 5.14 , using the redundant degree of freedom to allow for self motion of the manipulator and recording encoder data at various configurations. The link lengths were calculated using a least squares fit of the data using the forward kinematic model and isolating for $L_{r}$

$$
X_{e}=Q(q) L_{r}=\left[\begin{array}{ccccc}
c_{1} & c_{12} & c_{123} & c_{1234} & 0 \\
s_{1} & s_{12} & s_{123} & s_{1234} & 0 \\
0 & 0 & 0 & 0 & q_{1}+q_{2}+q_{3}+q_{4}
\end{array}\right] L_{r}
$$

where $\mathrm{X}_{\mathrm{e}}$ is the vector of the end location position and orientation and is equal to $[-0.292208,-0.167589,5.1051]^{\mathrm{T}}$ and $L_{r}=\left[\begin{array}{lllll}l_{1} & l_{2} & l_{3} & l_{4} & 1\end{array}\right]^{T}$, where $l_{n}$ is the length of link $n$. With $n$ data points, a least squares fit was performed with $L_{r}=\left(Q^{T} Q\right)^{-1}\left(Q^{T} X_{e}\right)$. The end effector was placed at $\mathrm{X}_{e}$ as defined above, and nine joint configurations were recorded. The results are shown in Table 5.2 with the errors plotted in Figure 5.15, Figure 5.16 and Figure 5.17. The errors were found by subtracting the measured end effector location from the results of each of the kinematic calibration at each of the analysis points from the forward kinematics of the manipulator. 


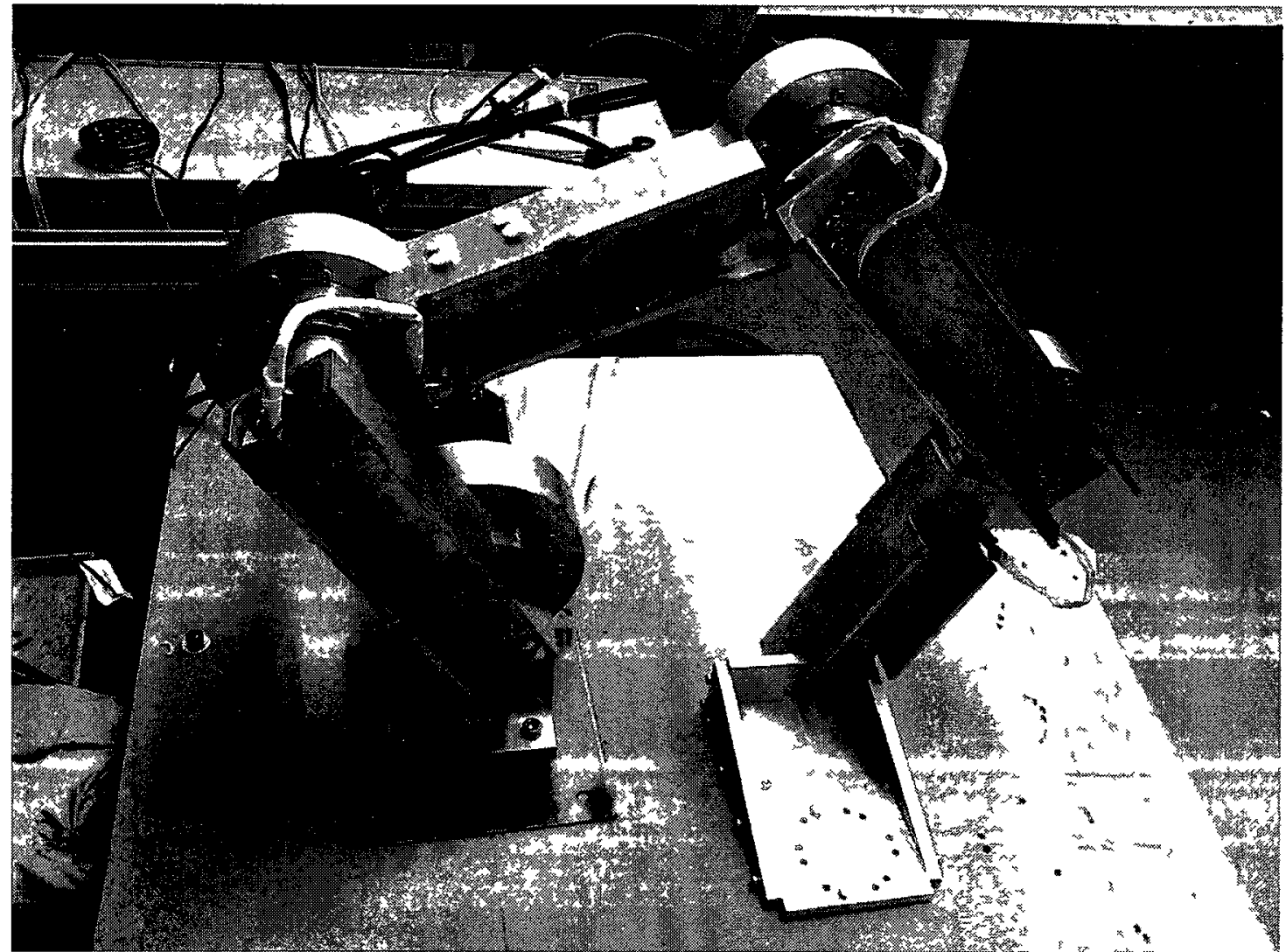

Figure 5.14: Kinematic Calibration Setup

The error plots do not show the error in the length determination but rather the error in the least squares fit of the data. The distance on a CNC machined joint component from the joint centre of Joint 1 to a measurable point on the structure was compared to data from the CAD drawings for which the error is within 0.0508 millimeters. From this location, the calipers and digital level were used to measure the distance on the bench to the center of the Force Torque sensor bolt hole pattern. To further improve the accuracy of the kinematic calibration a vision tracking system should be used. Alternatively multiple end effector locations with additional joint configurations should be used.

Though not exact, the lengths of the first three links are close to their optimized values from the manipulator optimization and component selection in 3.5. The fourth link was designed long as the end effector is not taken into account in this dimension. The base of the foot is built up approximately $110 \mathrm{~mm}$ above the base of the Force Torque 
sensor. At the point of construction of the I-beam link pieces it was unclear the dimensions of the end effector components and for this reason the fourth link was constructed too long.

Table 5.2: Results of Kinematic Calibration

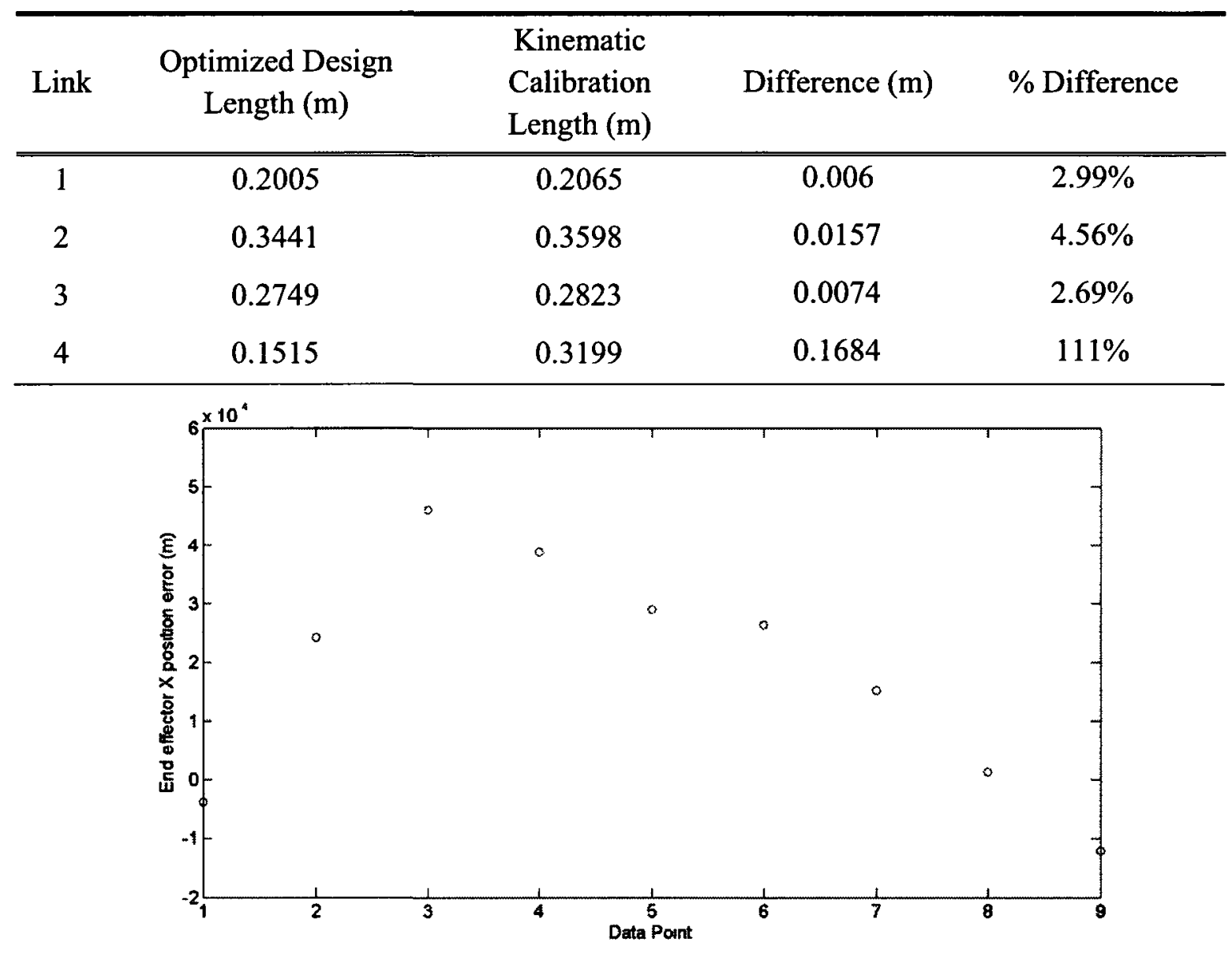

Figure 5.15: X Position Error 


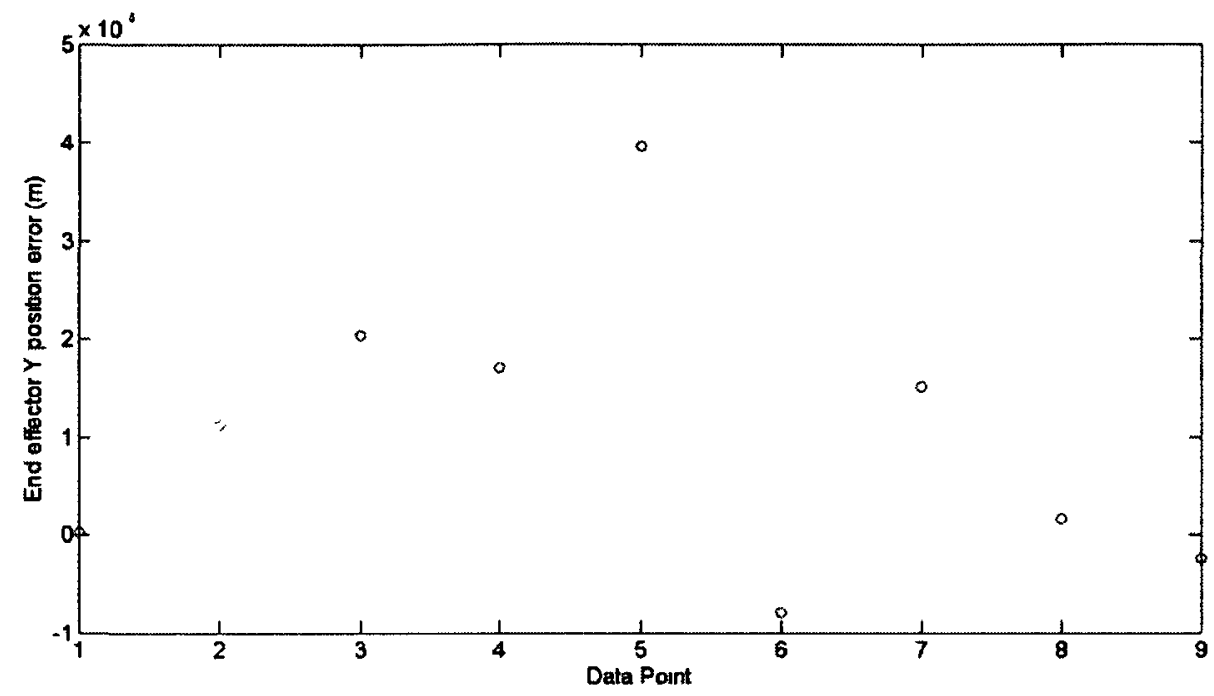

Figure 5.16: Y Position Error

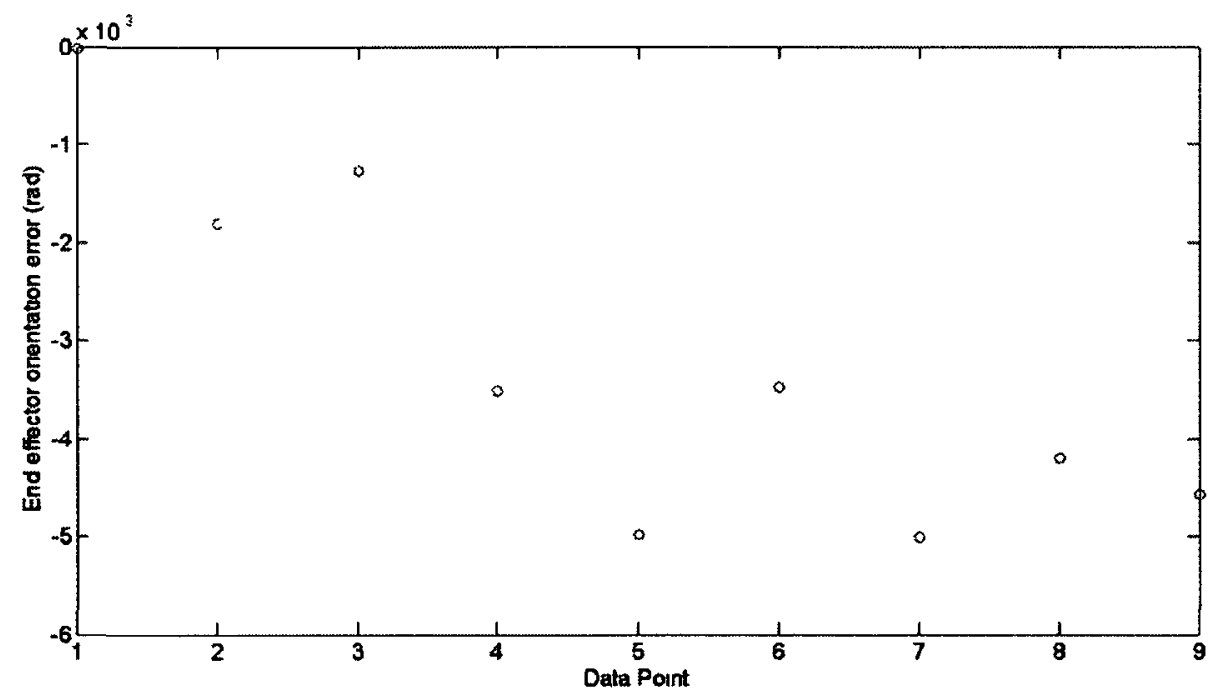

Figure 5.17: Angle Orientation Error

\subsection{Four Degrees-of-Freedom Dynamic Parameter Identification}

The results from the single degree of freedom dynamic and four degree of freedom kinematic calibrations were used as initial values for the assembled robot calibration. 
This calibration took into account the dynamics of the manipulator with the RegressorParameter formulation developed in [45]. A joint space trajectory was set of varying sinusoidal inputs with a varrety of amplitudes, frequencies and offsets biases. As with the single degree of freedom calibration, the joint positions and torques were recorded and the position data was filtered and integrated to determine the angular velocities and accelerations.

The linearized dynamic model with respect to the parameter vector $\phi$ is

$$
\tau=Y(q, \dot{q}, \ddot{q}) \phi
$$

Where $\tau$ is the applied torques, $\phi$ is the parameter vector and $Y$ is the regressor matrix from [45]. As with the single degree of freedom calibration, the coulomb friction $u_{k}$ could not be isolated from the regressor $Y$. In this case, $u_{k}$ was set as the values from the $1 \mathrm{DoF}$ calibration. Using the $N$ recorded data points from the motion the dynamic parameters can be identified using a least squares fit of

$$
\begin{gathered}
\tau=Y \phi \\
\left\{\begin{array}{c}
\left(\tau_{r}\right)_{1} \\
\left(\tau_{r}\right)_{2} \\
\vdots \\
\left(\tau_{r}\right)_{N}
\end{array}\right\}=\left[\begin{array}{c}
Y(q, \dot{q}, \ddot{q})_{1} \\
Y(q, \dot{q}, \ddot{q})_{2} \\
\vdots \\
Y(q, \dot{q}, \ddot{q})_{N}
\end{array}\right] \phi
\end{gathered}
$$

as

$$
\hat{\phi}=\left(Y^{T} Y\right)^{-1}\left(Y^{T} \tau\right)
$$

with the recalculated torques from the calibrated properties as

$$
\hat{\tau}=Y \hat{\phi}
$$

The calibration error is the difference between the applied torques and the torques found using the dynamic model and the updated parameter values. The calibrated values of $\phi$ are: 


$\hat{\phi}=\left\{\begin{array}{c}\left(I_{1}+l_{1}^{2} m_{2}+l_{1}^{2} m_{3}+l_{1}^{2} m_{4}+l_{C 1}^{2} m_{1}+I_{e f f 1}\right) \\ \left(I_{3}+l_{3}^{2} m_{4}+l_{C 3}^{2} m_{3}\right) \\ \left(I_{4}+l_{C 4}^{2} m_{4}\right) \\ \left(I_{2}+l_{2}^{2} m_{3}+l_{2}^{2} m_{4}+l_{C 4}^{2} m_{2}\right) \\ \left(l_{C 4} g m_{4}\right) \\ \left(l_{2} l_{3} m_{4}+l_{2} l_{C 3} m_{3}\right) \\ \left(l_{2} g m_{3}+l_{2} g m_{4}+l_{C 2} g m_{2}\right) \\ \left(l_{3} l_{C 4} m_{4}\right) \\ \left(l_{1} g m_{2}+l_{1} g m_{3}+l_{1} g m_{4}+l_{C 1} g m_{1}\right) \\ \left(l_{31} g m_{4}+l_{C 4} g m_{3}\right) \\ \left(l_{1} l_{C 4} m_{4}\right) \\ \left(l_{2} l_{C 4} m_{4}\right) \\ \left(l_{1} l_{2}+l_{1} l_{2} m_{4}+l_{1} l_{C 1} m_{2}\right) \\ \left.l_{1} l_{3}+l_{1} l_{C 3} m_{3}\right) \\ B_{e f f 1} \\ B_{e f f 2} \\ B_{e f f 3} \\ B_{e f f 4} \\ v_{C 1} \\ v_{C 2} \\ v_{C 3} \\ v_{C 4} \\ I_{e f f 2} \\ I_{e f 3} \\ I_{e f f 4} \\ 0.3221 \\ 1.8100 \\ 7.7466 \\ 0.5864 \\ 39.9933 \\ 0.4910 \\ 41.4924 \\ 20.6041 \\ 0.1637 \\ 0.2693 \\ 0.8439 \\ 0.7446 \\ 20.4829 \\ 39.6782 \\ 16.7666 \\ 5.03059 \\ 19.8307 \\ 21.7988 \\ 13.1578 \\ 4.3201 \\ 4.2331 \\ 1.7297 \\ 0.5267\end{array}\right\}$

Figure 5.18 through Figure 5.21 show the joint torques through the calibration motion, Figure 5.22 through Figure 5.25 show the errors. The maximum torque capacities of each of the joints is listed in Table 5.3 and compared to the maximum error in the torque applied during this calibration. The errors are all less than 5\% and these locations represent points where the manipulator changed direction. 


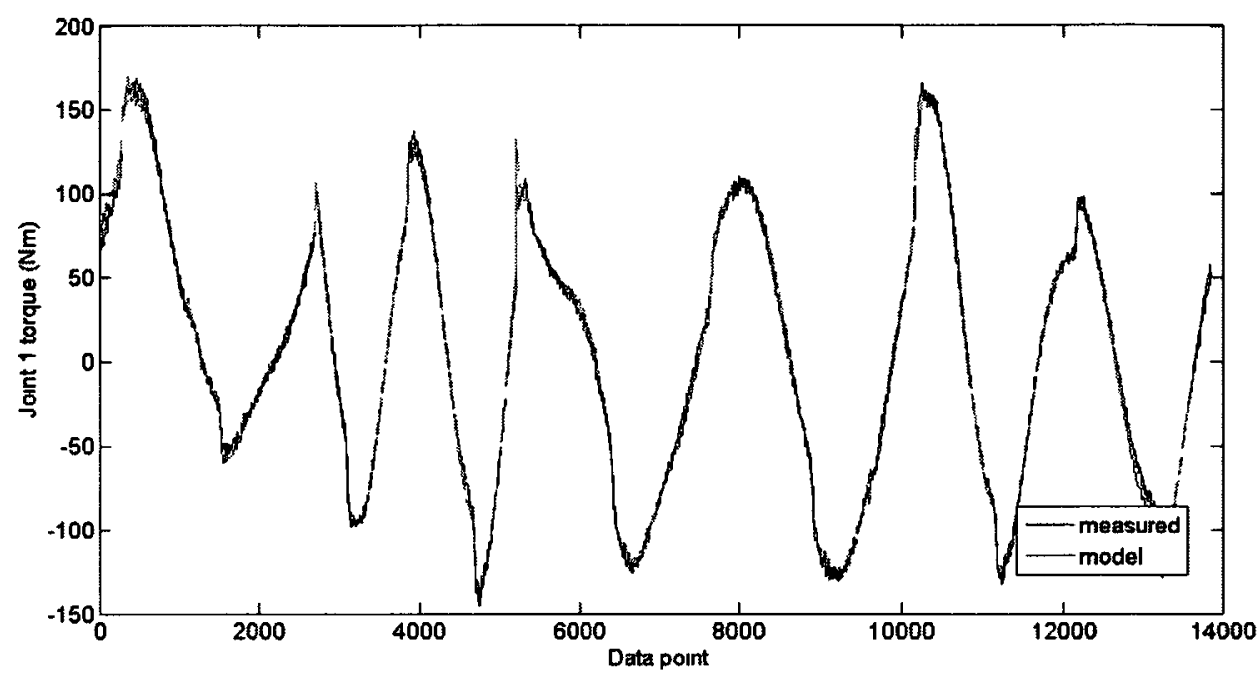

Figure 5.18: Measured and Modeled Joint 1 Torques

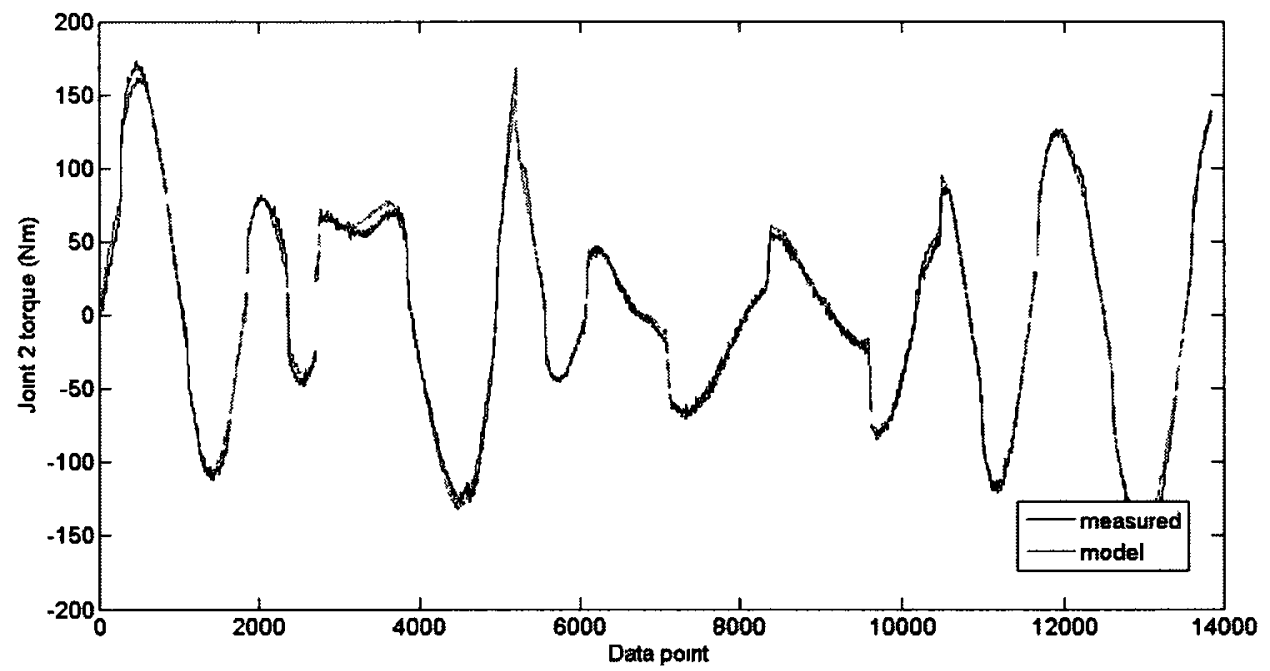

Figure 5.19: Measured and Modeled Joint 2 Torques 


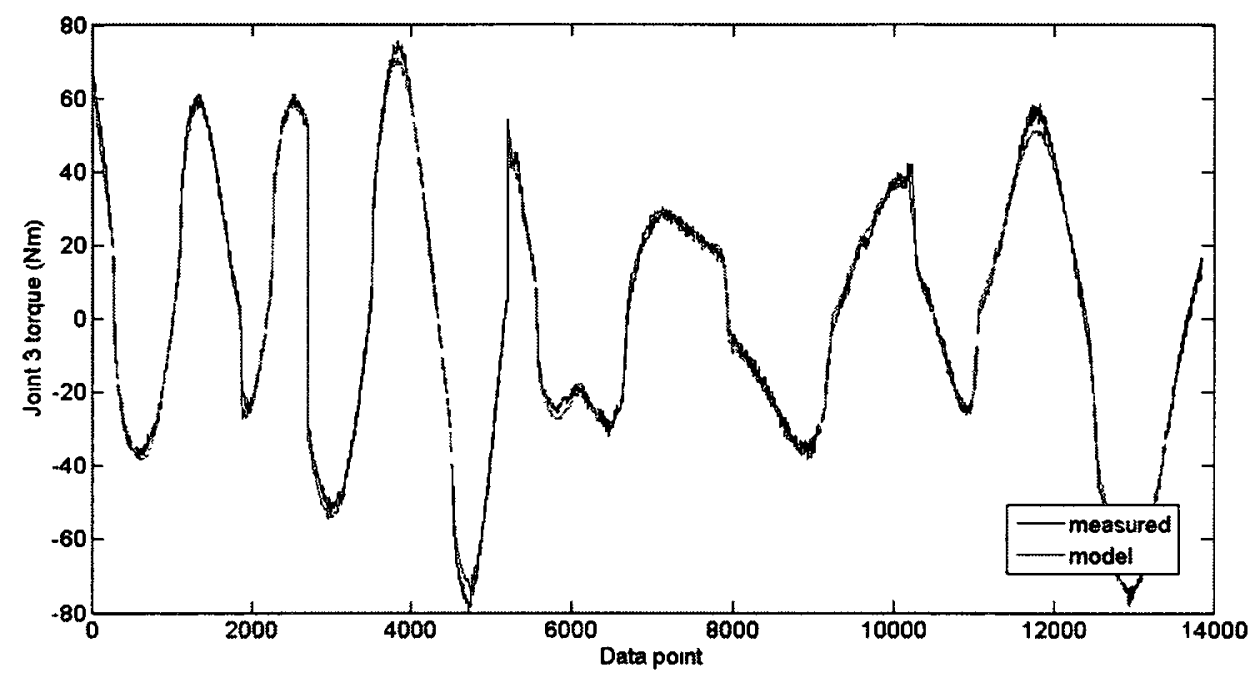

Figure 5.20: Measured and Modeled Joint 3 Torques

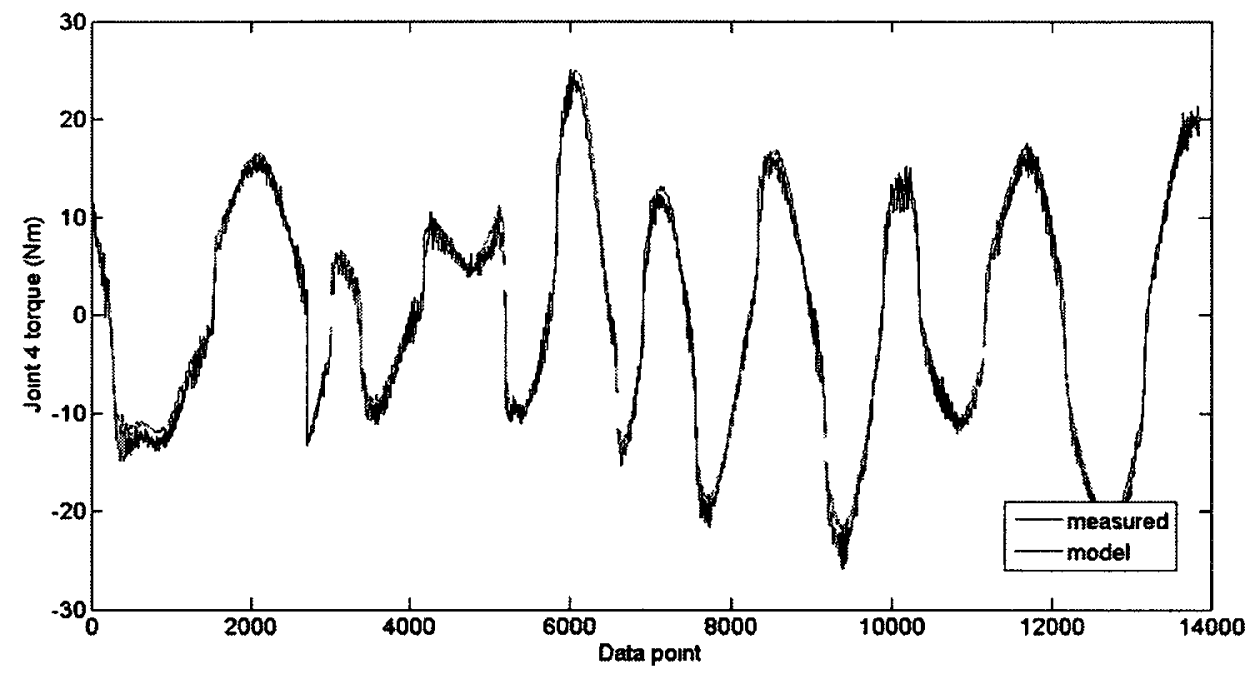

Figure 5.21: Measured and Modeled Joint 4 Torques 

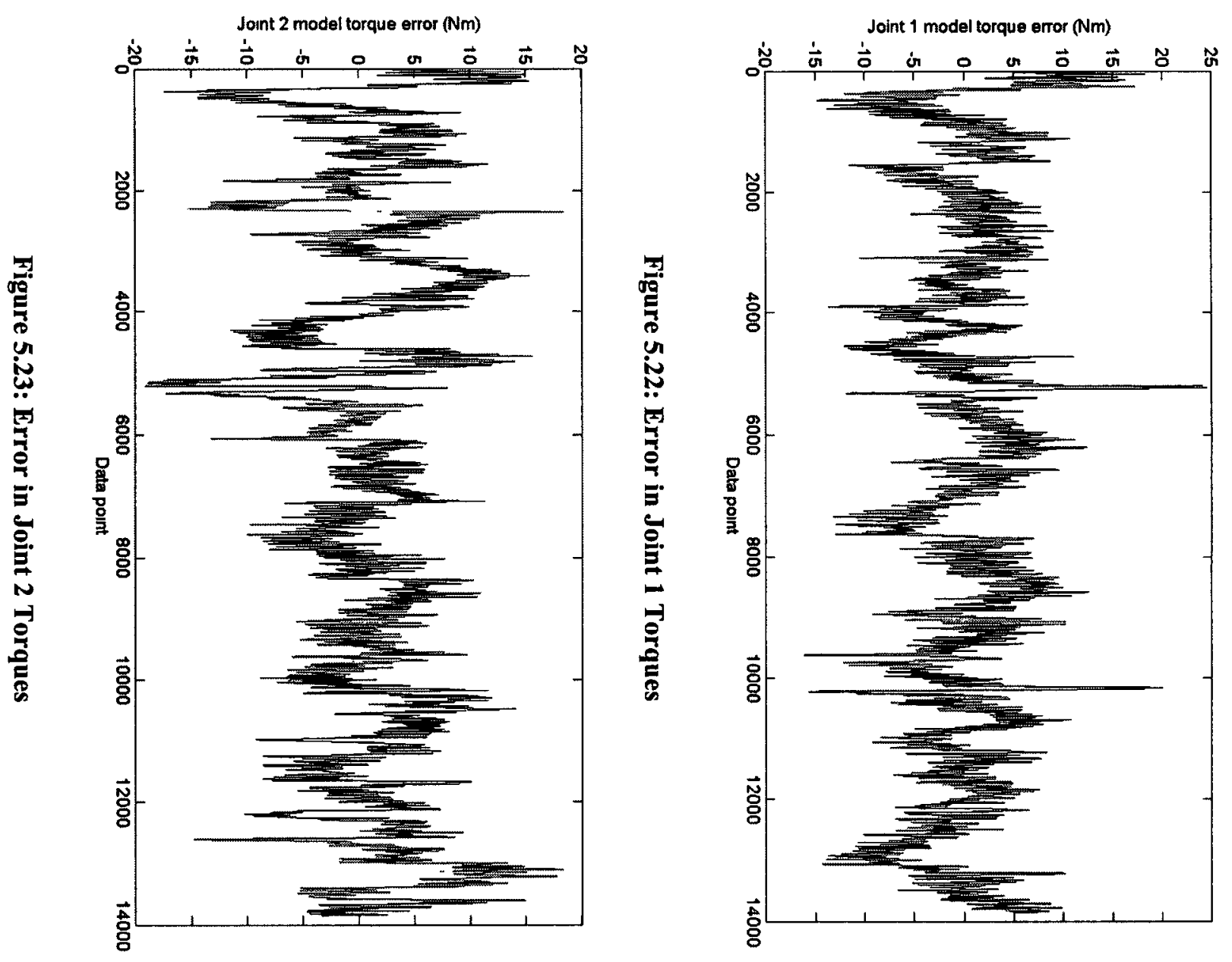


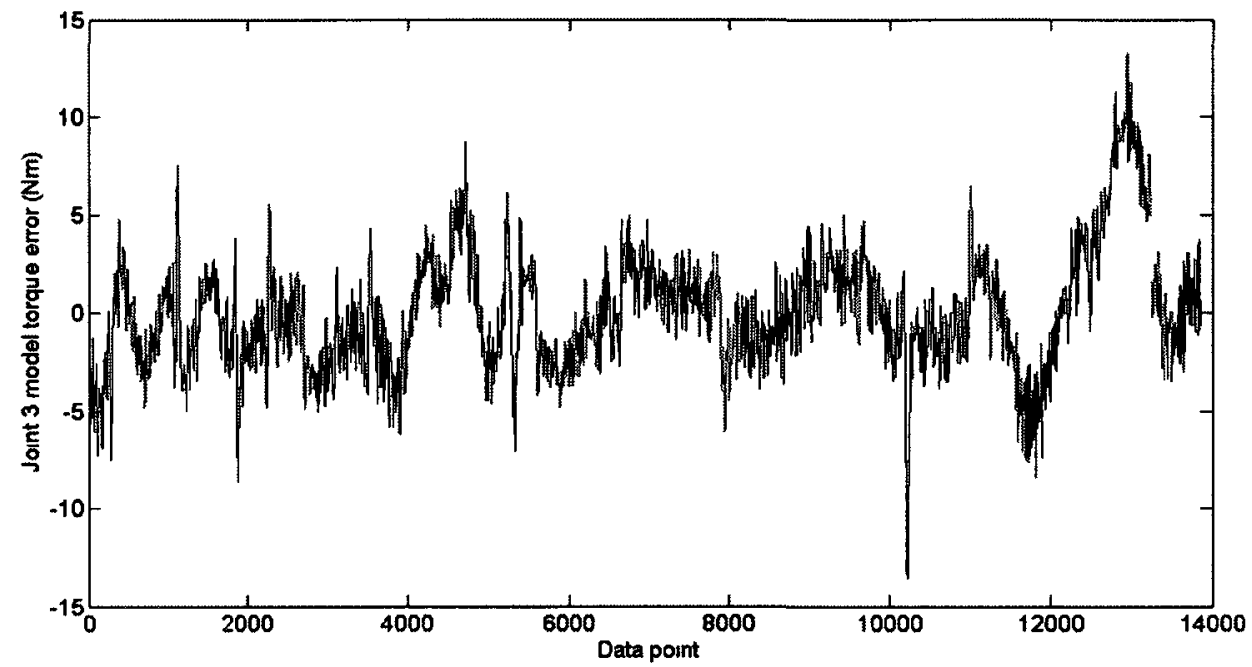

Figure 5.24: Error in Joint 3 Torques

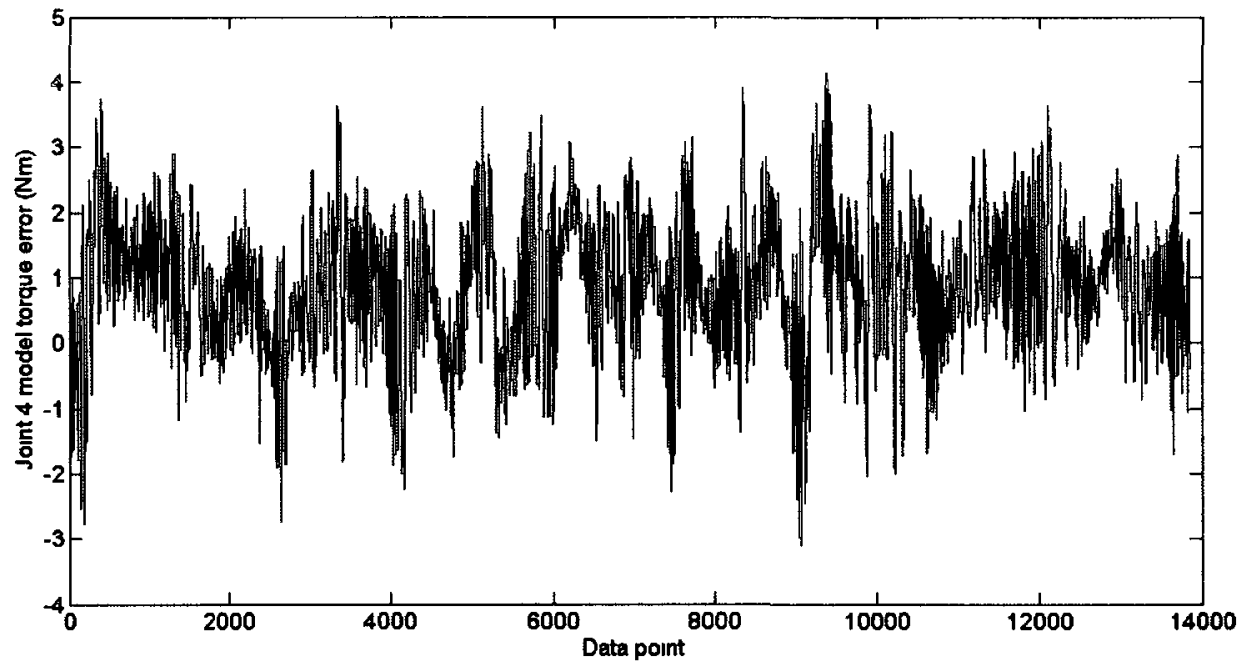

Figure 5.25: Error in Joint 4 Torques 
Table 5.3: Torque Capacity of Joints

\begin{tabular}{cccc}
\hline & $\begin{array}{c}\text { Maximum Torque } \\
\text { Capacity }\end{array}$ & Torque Error & $\begin{array}{c}\text { Percent of } \\
\text { Maximum Torque }\end{array}$ \\
\hline Joint 1 & $892 \mathrm{Nm}$ & $24.48 \mathrm{Nm}$ & 2.74 \\
Joint 2 & $892 \mathrm{Nm}$ & $19.11 \mathrm{Nm}$ & 2.14 \\
Joint 3 & $309 \mathrm{Nm}$ & $13.55 \mathrm{Nm}$ & 4.39 \\
Joint4 & $191 \mathrm{Nm}$ & $4.141 \mathrm{Nm}$ & 2.17 \\
\hline
\end{tabular}

\subsection{Experimentation}

Upon completion of the design process of the mechanical, electrical and control systems of the ViGRR platform the system was tested to ensure that the platform would operate as designed. The remainder of this chapter outlines the tests performed on the system. First, the platform was controlled in joint space, where each joint is given a particular starting and final orientation and the controller creates a smooth motion between the two positions. Second, trajectory trackıng tests were performed in the task space, where a motion based on the position of the end effector was performed. Lastly, the admittance model of the controller was tested using the force feedback of the system. In the last experiment, the user was asked to trace a trajectory on the screen while manipulating the end effector.

Figure 5.26 shows the control strategy for both the joint and task based experiments. In the joint level control, the desired joint positions are input to the joint space trajectory generator, which develops a smooth joint level motion from the current configuration to the final configuration. This trajectory is input to the controller where the trajectory controller monitors the encoder output from the motors and applies the modelled torques from the inverse dynamics to achieve the desired trajectory. In the task space control, the desired trajectory of the end effector, is fed through the redundancy resolution inverse kinematics scheme to determine the desired joint space controls to achieve the desired task space motion, in the case of the experiments listed below, a circular trajectory. The joint space control is fed to the physical system, again the encoder data is fed back to 
trajectory controller which uses the robot dynamics to determine the necessary torques to supply to the actuators. In a force control mode, the force torque sensor is included in the sensory feedback.

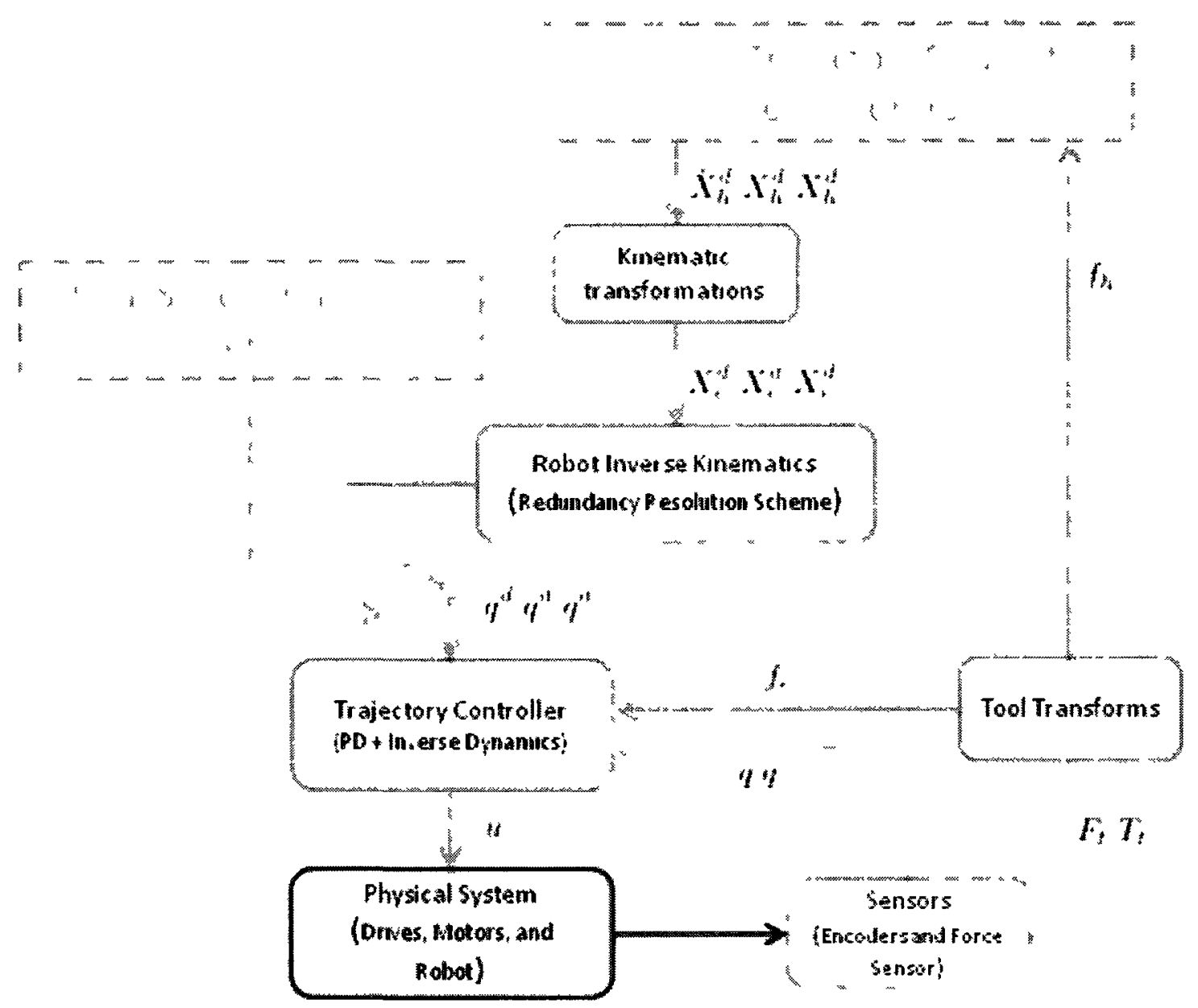

Figure 5.26: Control Strategy for Joint and Task Space Experiment

\subsubsection{Joint Space Experiment}

When operating in joint space control, the user inputs the desired joint angles for each of the four joints. The controller creates a fifth order spline fit of the trajectory from the current location to the desired location, with a five second start to finish time. The angles used in this experiment are listed in Table 5.4. Figure 5.27 through Figure 5.30 show the 
tracking of the actual motion compared to the desired. Figure 5.31 through Figure 5.34 show the error of each of the joints throughout the motion. The maximum and average error results during the motion for each of the joints are compiled in Table 5.5.

Table 5.4: Joint Space Motion Values

\begin{tabular}{cccc}
\hline Joint & Initial Angle & Final Angle & Total Travelled \\
\hline 1 & 120 & 20 & 100 \\
2 & -90 & 90 & 180 \\
3 & 30 & 80 & 50 \\
4 & -30 & 60 & 90 \\
\hline
\end{tabular}

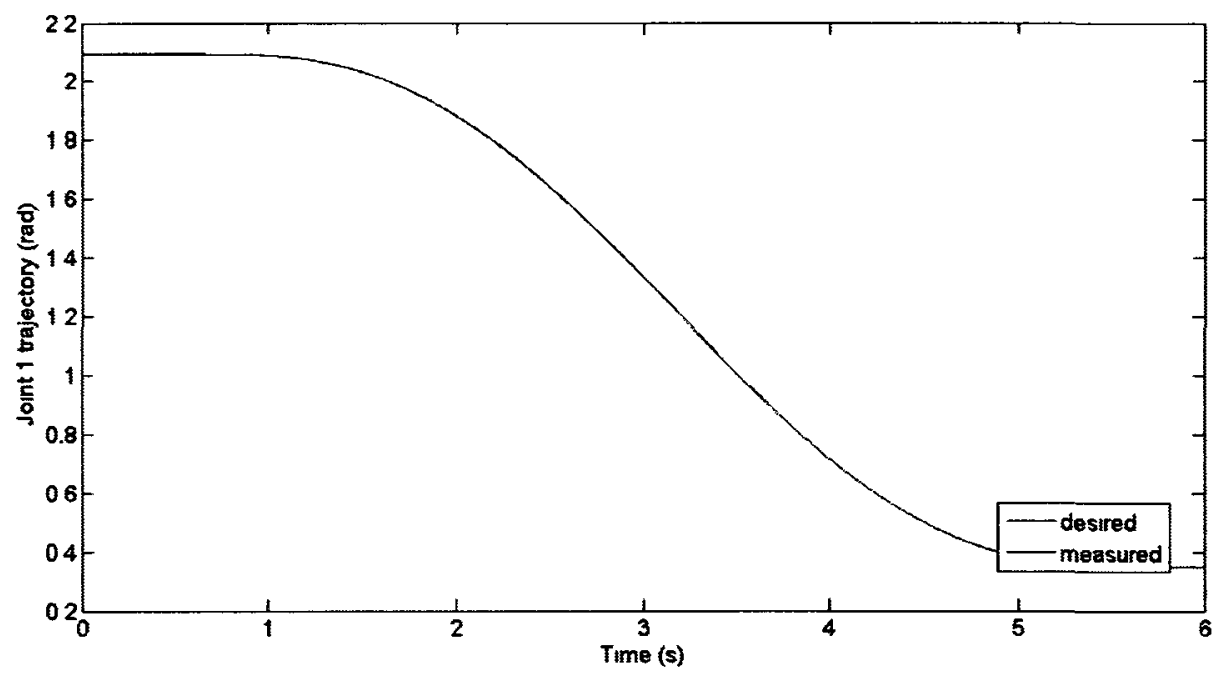

Figure 5.27: Joint Trajectory - Joint 1 


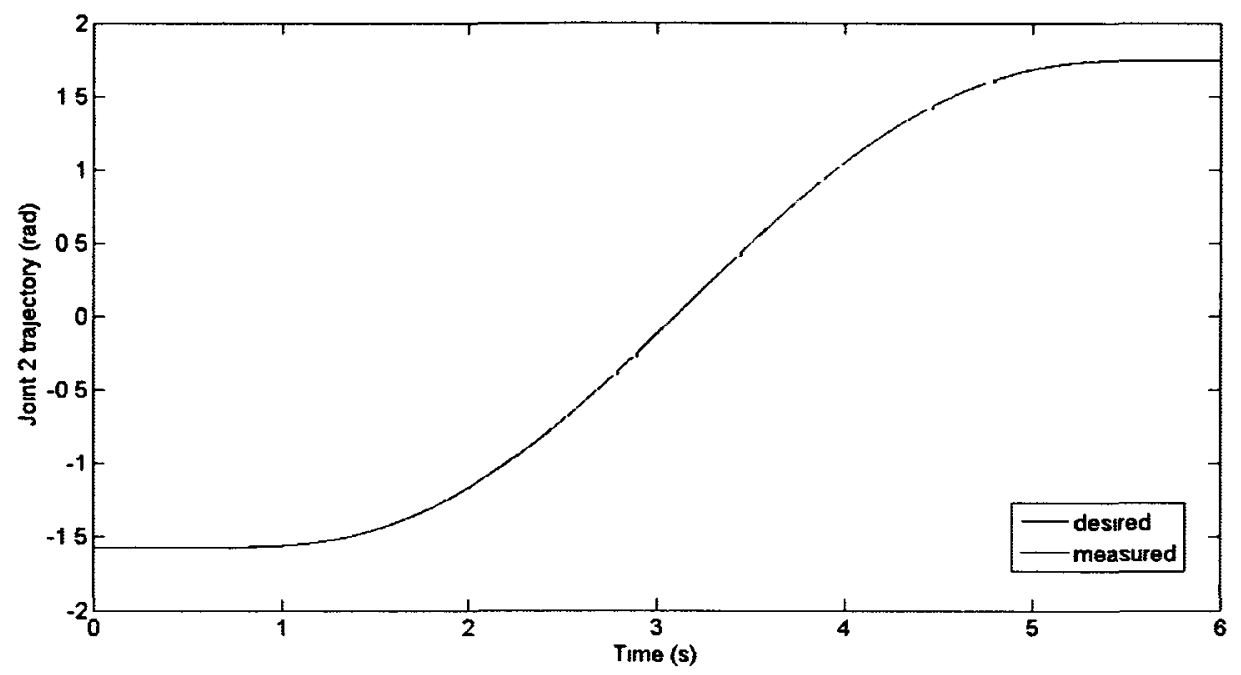

Figure 5.28: Joint Trajectory - Joint 2

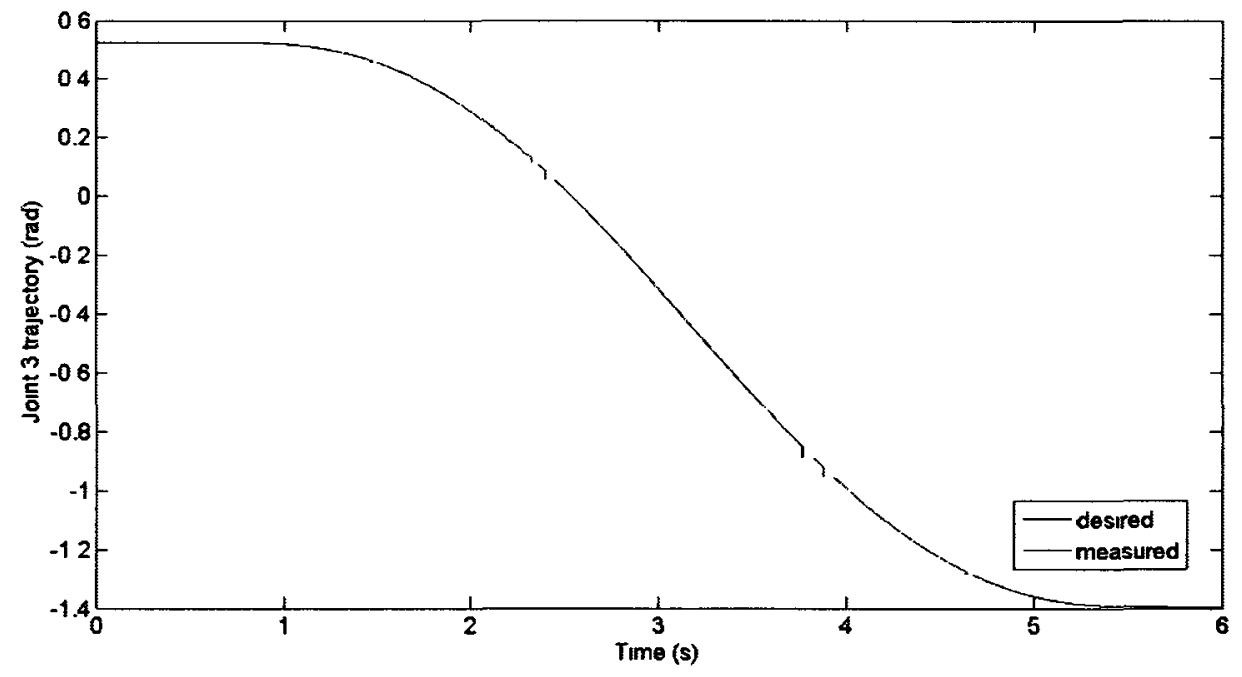

Figure 5.29: Joint Trajectory - Joint 3 


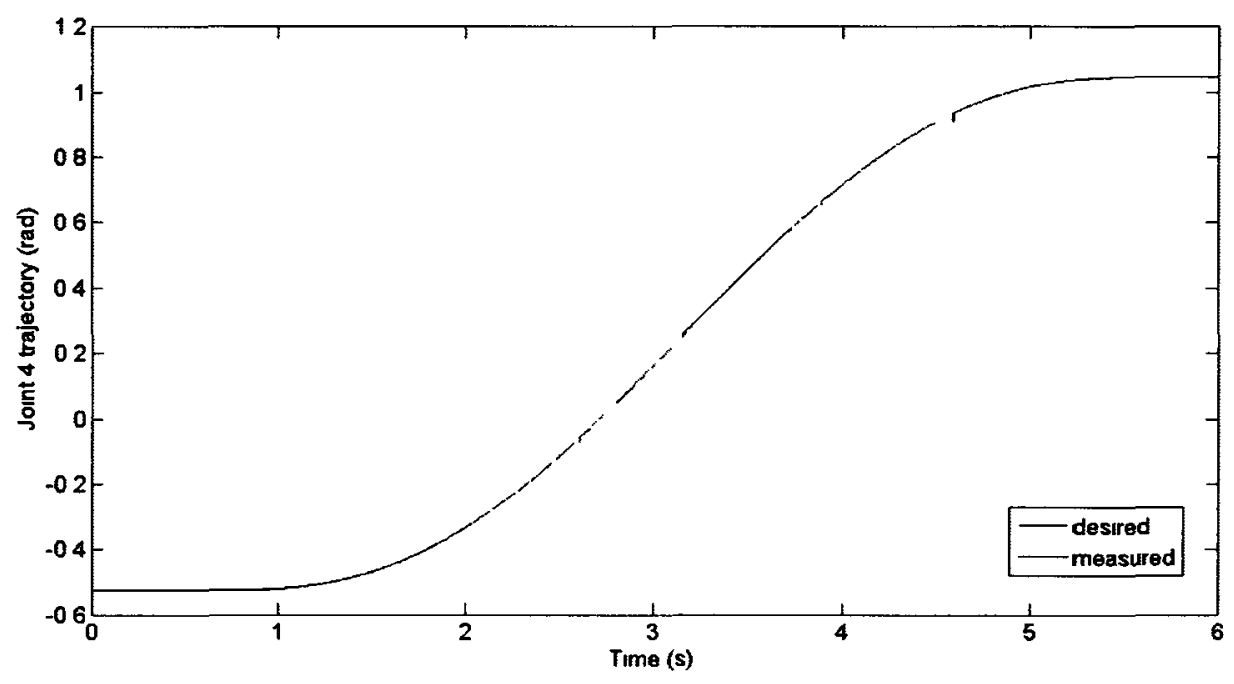

Figure 5.30: Joint Trajectory - Joint 4

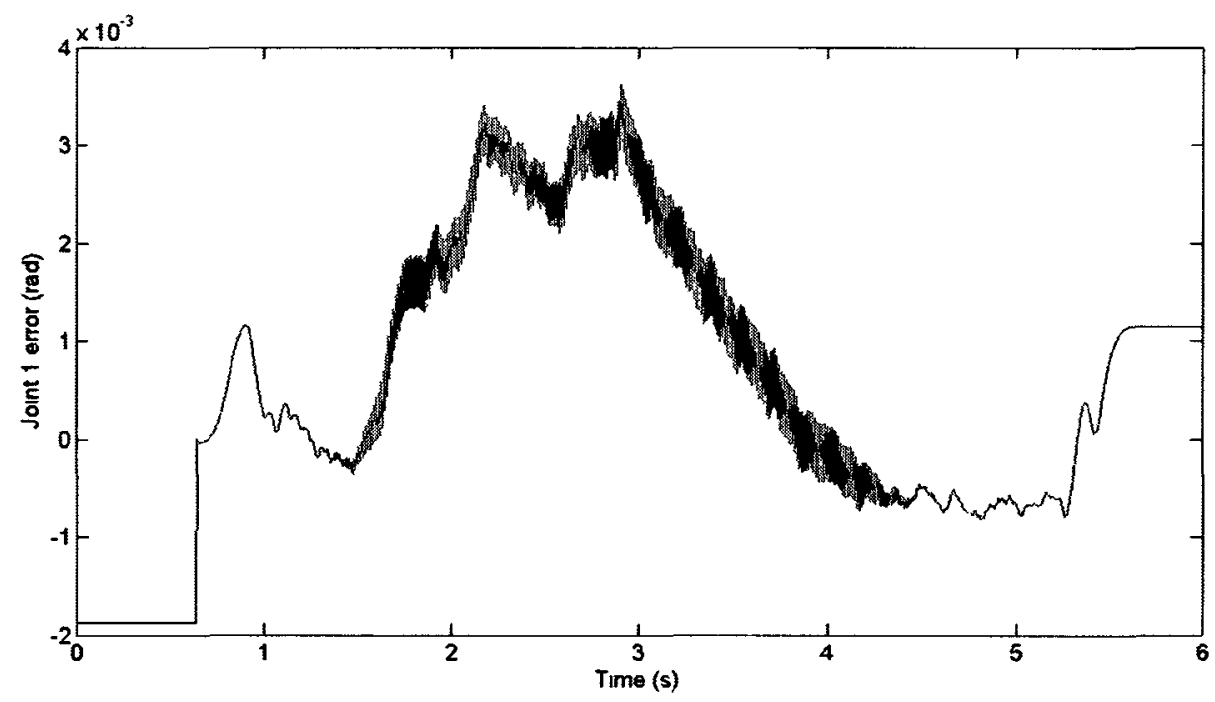

Figure 5.31: Joint Trajectory Error- Joint 1 


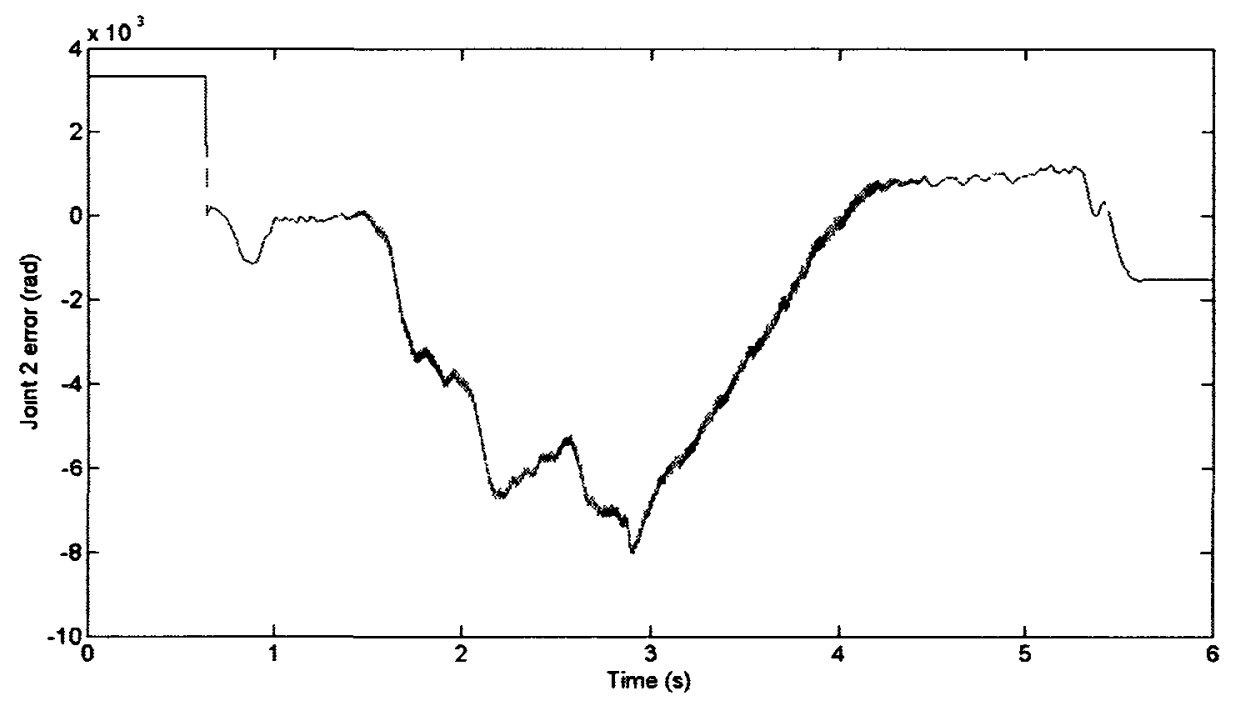

Figure 5.32: Joint Trajectory Error- Joint 2

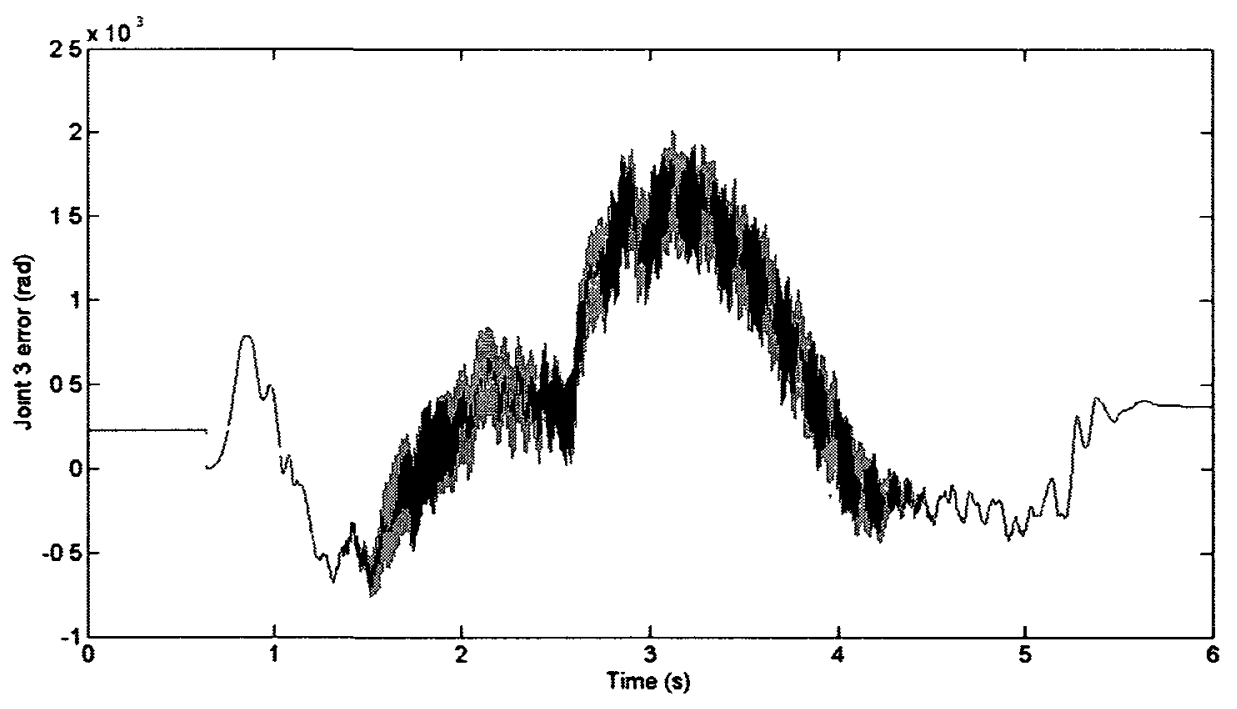

Figure 5.33: Joint Trajectory Error- Joint 3 


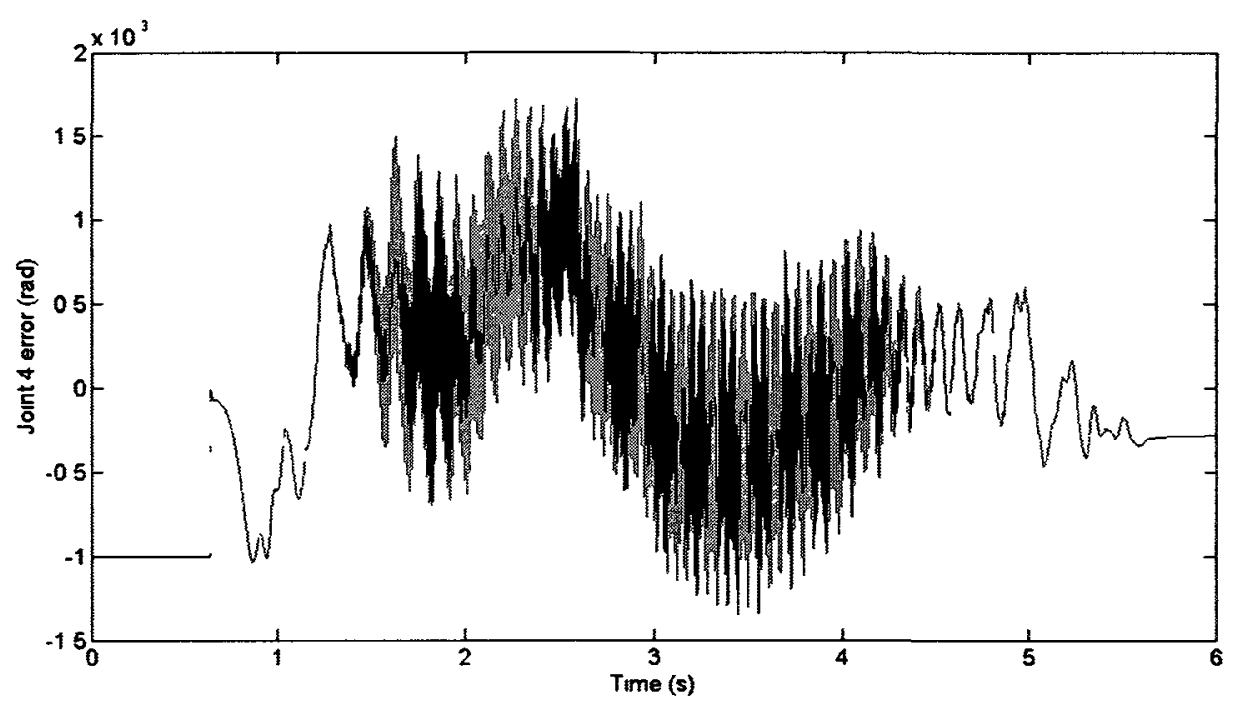

Figure 5.34: Joint Trajectory Error - Joint 4

Table 5.5: Error Results

\begin{tabular}{ccc}
\hline & Avg Error (deg) & Max Error (deg) \\
\hline Joint 1 & 0.0664 & 0.2079 \\
Joint 2 & 0.1436 & 0.4602 \\
Joint 3 & 0.0311 & 0.1155 \\
Joint 4 & 0.0263 & 0.0989 \\
\hline
\end{tabular}

The error present during the motion is on the order of $10^{-3}$ radians. The error spikes at the midpoint of the trajectory when the velocities are the highest and there are high accelerations. This may be due to modelling errors. A more variable set of motions taking into account lower speed motions during the parameter identification could lead to more accurate parameters. Further tuning of the $d$ gain of the controller could also decrease the noise. An accuracy of less than half a degree maximum error during the motion is most acceptable. 


\subsection{Task Space Experiment}

The inverse kinematics and the redundancy resolution scheme were used to determine the joint controls for a circular end effector trajectory. As in the joint space control, a fivesecond fifth order polynomial blend was generated to ramp the end effector from the current location to the circular trajectory. Desired angular positions, velocities and accelerations were then implemented to trace the circular shape. The parameters of the trajectory are listed in Table 5.6.

Table 5.6: Task Space Circle Parameters

\begin{tabular}{rc}
\hline Parameter & Value \\
\hline Center of Circle $(\mathrm{x}, \mathrm{y})(\mathrm{m})$ & $(0.65,0.45)$ \\
Radius (m) & 0.15 \\
Period of Blending Trajectory (s) & 5 \\
End Effector Angle Bias (rad) & 0 \\
End Effector Angle Amplitude (rad) & 0.25 \\
\hline
\end{tabular}

Figure 5.35 shows the tracking of the circular trajectory, including the 5 second lead in curve. Figure 5.35 through Figure 5.38 show the accuracy of the tracking while Figure 5.39 through Figure 5.42 show the tracking error for Figure 5.35 through Figure 5.38 respectively. Table 5.7 shows the maximum and average errors of the end effector position and orientation. Though there are oscillations of the end effector location throughout the trajectory, the maximum error does not exceed a millimeter in the $\mathrm{X}$ direction and is slightly larger in the $\mathrm{Y}$. The error pattern is highly repetitious, repeating each cycle of the motion, this indicates an inaccuracy in the parameters of the manipulator as the dynamics is not properly accounting for the desired motion.

As the position feedback control in this mode is performed at the joint level, there could be errors in the tracking of the end effector location, as an inaccuracy in the kinematic calibration would cause errors in the inverse kinematics of the dynamic model. Additionally, the errors reported are not based on the absolute end effector position but is 
rather an assessment of the controller as the forward kinematics of the manipulator model were used to determine the desired location. As the position errors for the task are submillimeter, tracking of a desired location under gait loading would be sufficiently accurate to articulate the leg in position control rehabilitation.

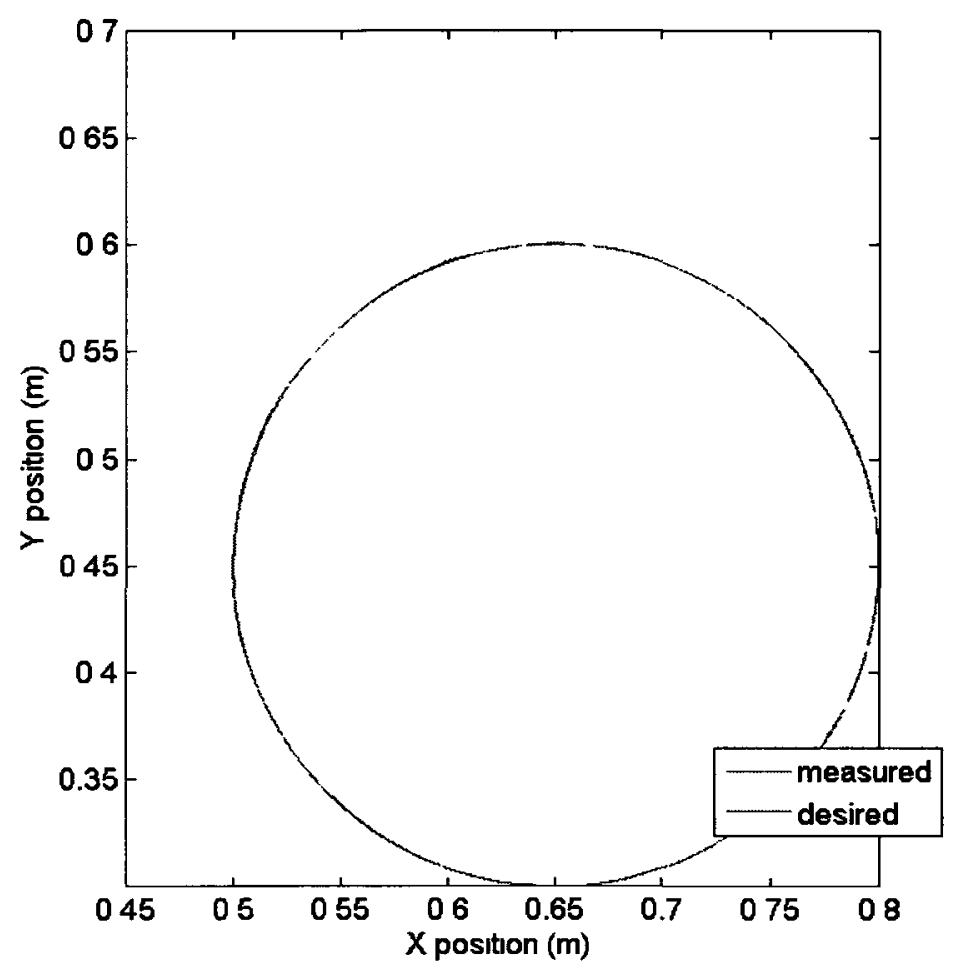

Figure 5.35: End Effector Position Tracking 


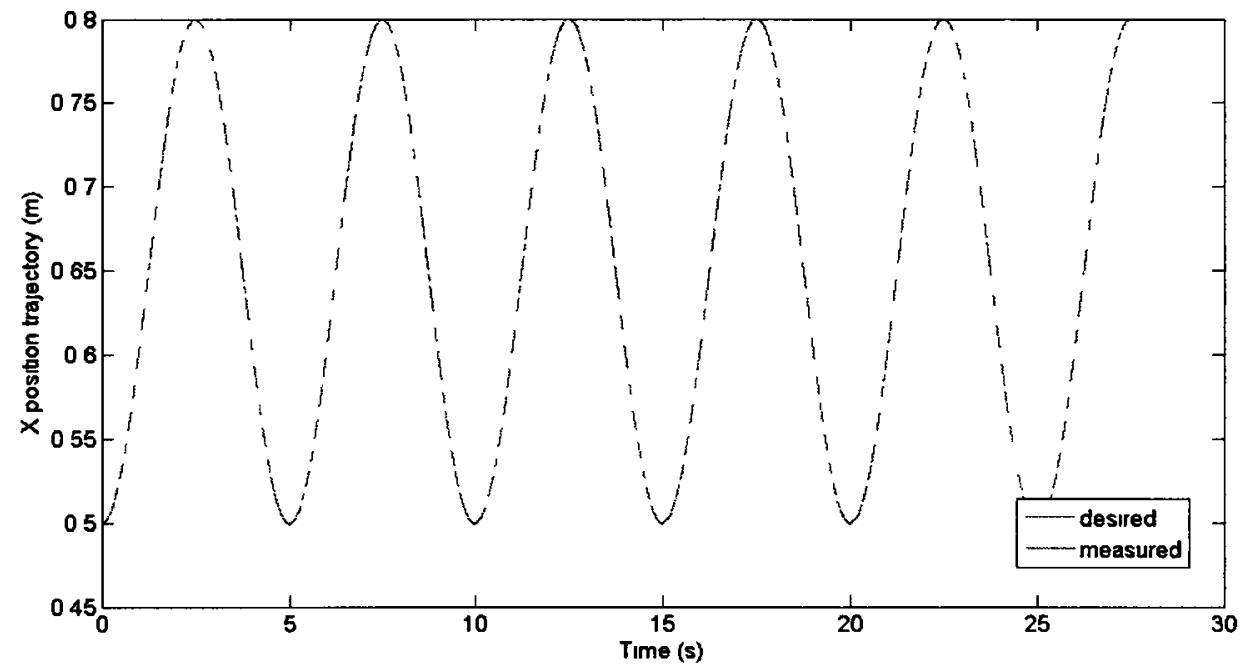

Figure 5.36: End Effector Tracking - X

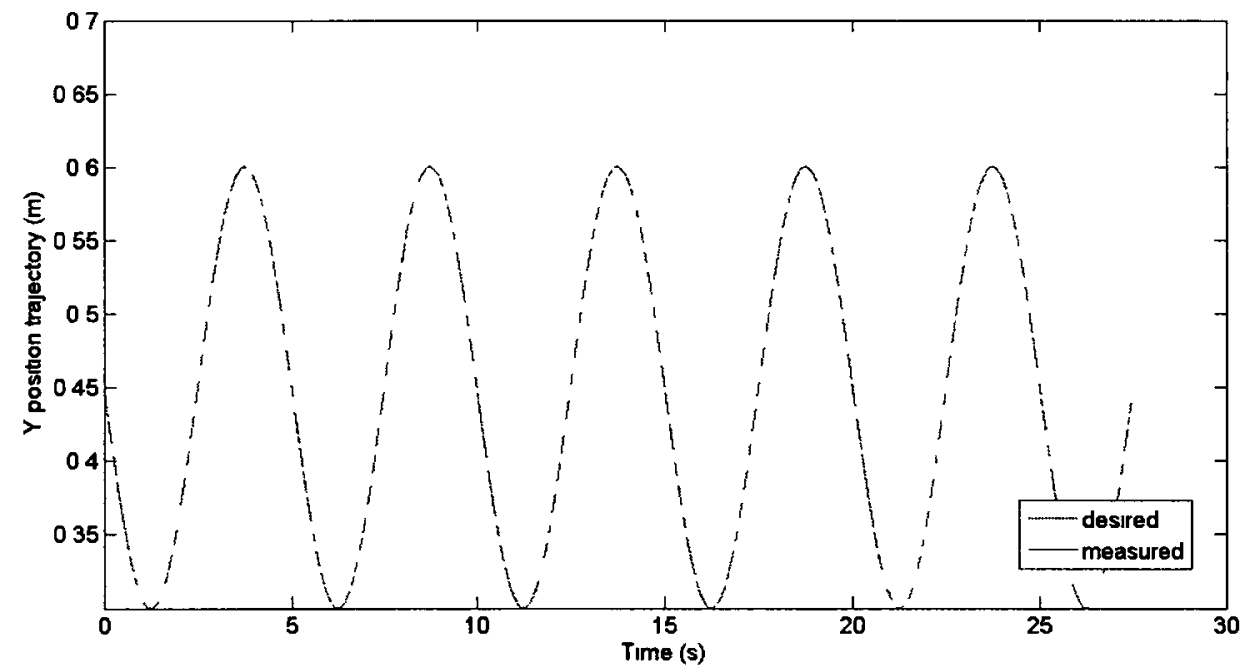

Figure 5.37: End Effector Tracking - Y 


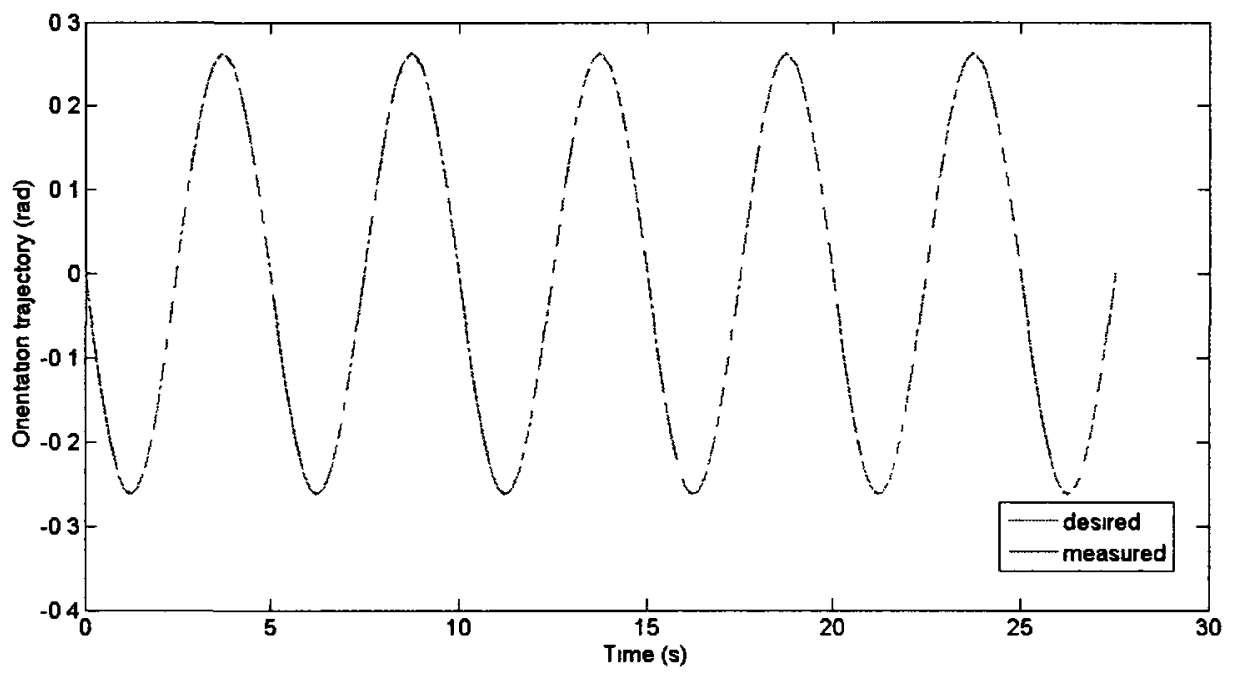

Figure 5.38: End Effector Tracking - Orientation

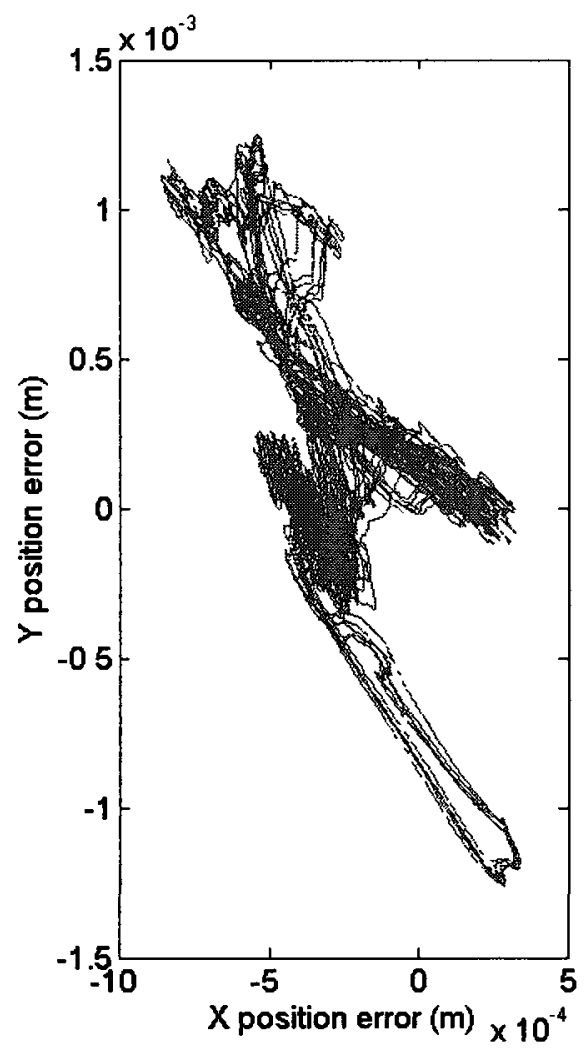

Figure 5.39: End Effector Tracking Error 

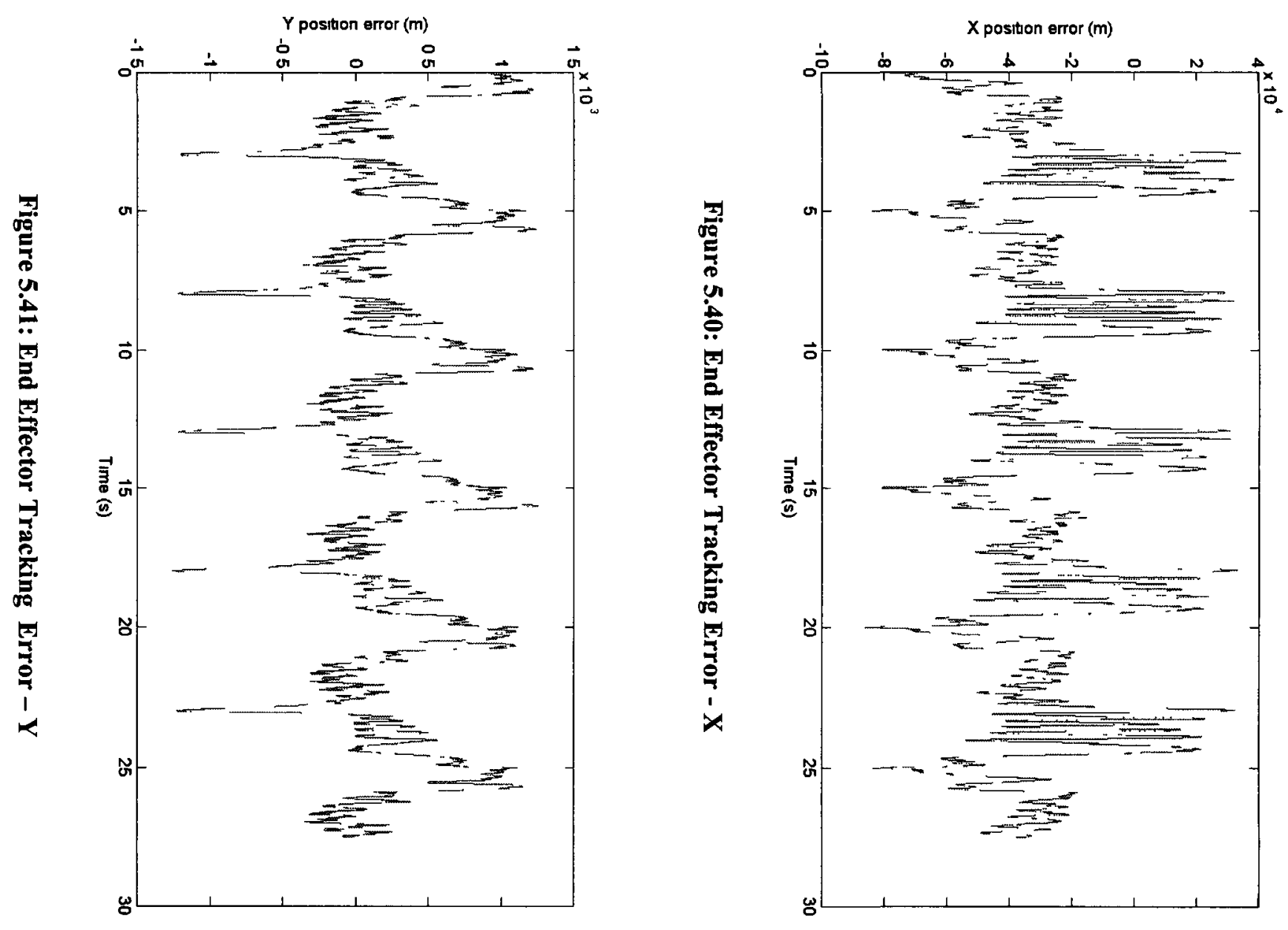


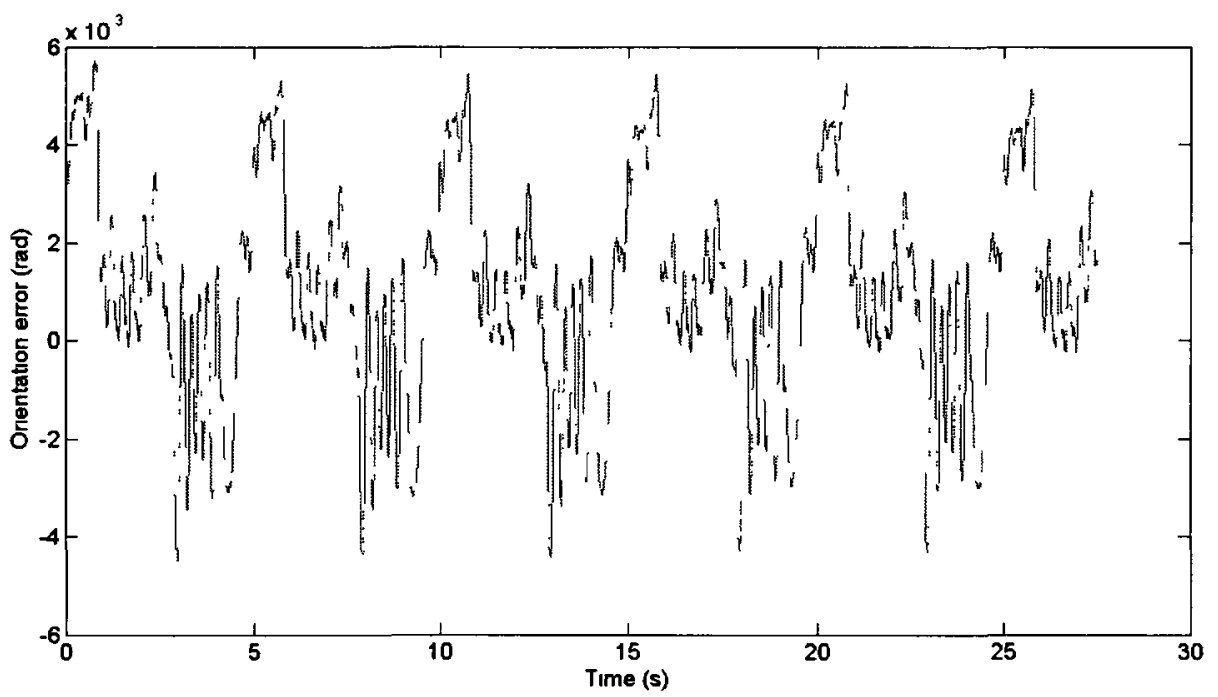

Figure 5.42: End Effector Tracking Error - Orientation

Table 5.7: End Effector Tracking Error Results

\begin{tabular}{ccc}
\hline & Avg Error & Max Error \\
\hline \hline $\mathrm{X}$ & $0.326 \mathrm{~mm}$ & $0.864 \mathrm{~mm}$ \\
$\mathrm{Y}$ & $0.375 \mathrm{~mm}$ & $1.266 \mathrm{~mm}$ \\
Theta & $0.1165 \mathrm{deg}$ & $0.3339 \mathrm{deg}$ \\
\hline
\end{tabular}

\subsection{Admittance Control Experiment}

Using the admittance force feedback controller developed in [45] along with a visual aide, the robotic system was controlled through a hand piece attached to the Force Torque sensor on the end effector of the robot. This experiment was performed to test the force control model of the controller and determine fidelity of the system by having the user trace a trajectory on the screen through interaction with the robot. The test setup is shown in Figure 5.43, the screen in the background displays the location of the end effector in 2D space and also the desired trajectory for the user to follow, Figure 5.44. 


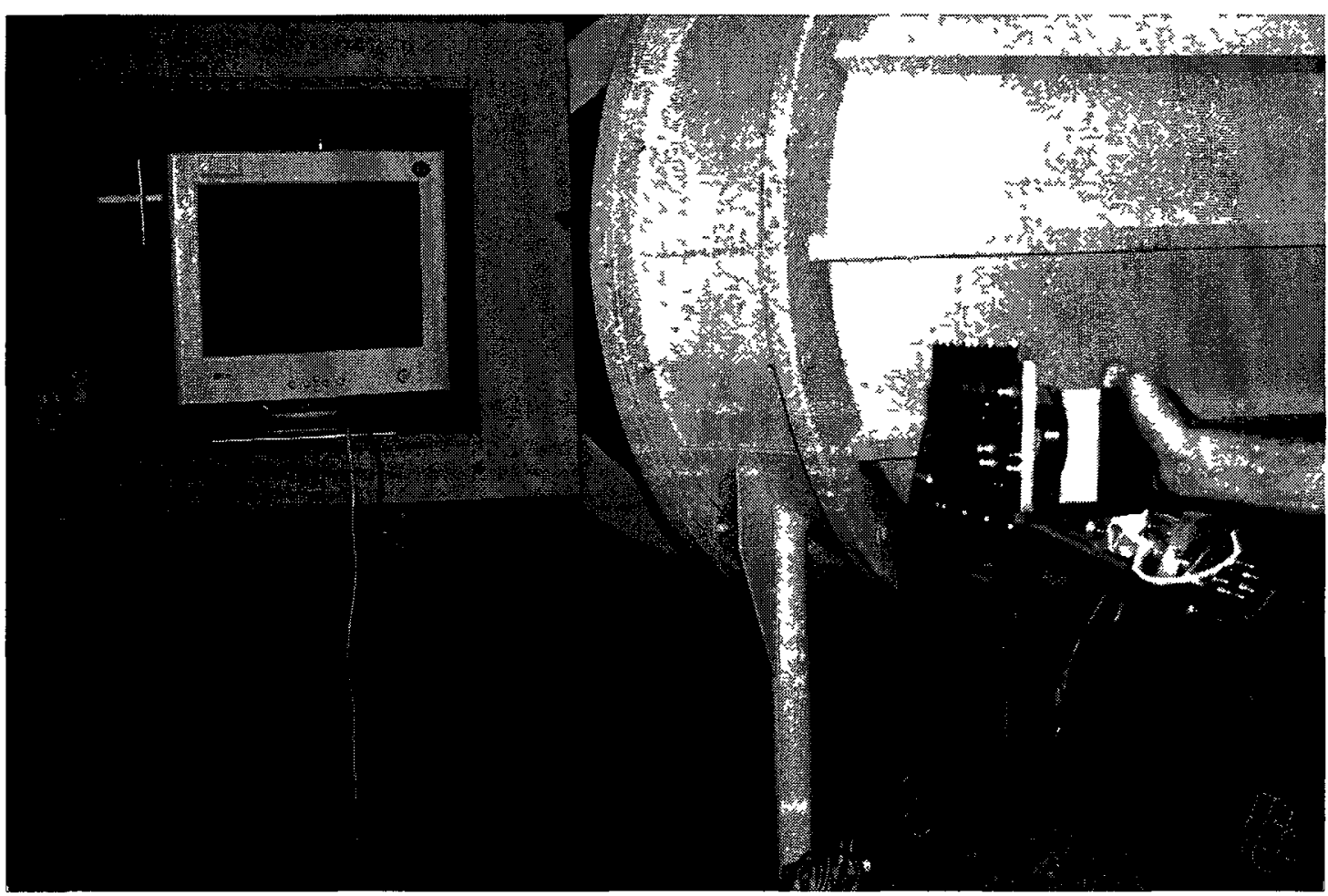

Figure 5.43: Admittance Control Setup

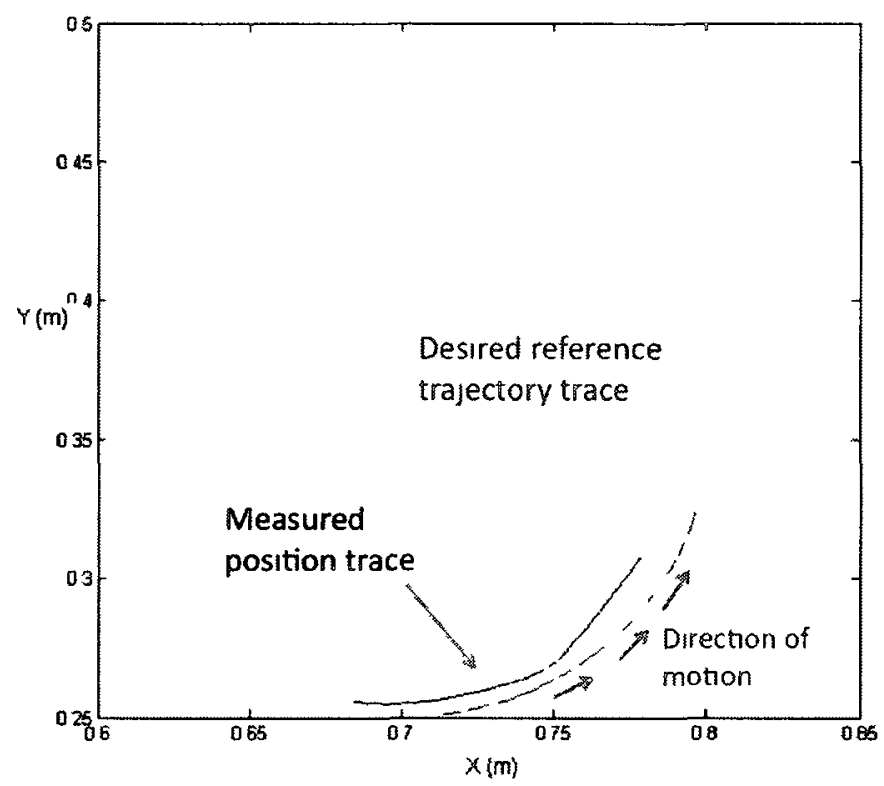

Figure 5.44: User Interface for Admittance Control Mode 
The admittance controller used for this experiment was developed in [45] and the controller process is shown in Figure 5.45. Similar to the process of the joint and task space design described above, the system outputs the current location of the end effector onto the screen through application of the forward kinematics to the encoder data. The dotted bounding box contains the admittance controller.

The parameters of the controller are given in Table 5.8, the inertia of the end effector, $M$, the damping, $B$, and the restorative stiffness, $K$. The desired forces, velocities and accelerations of the controller were all set to zero. The position indicated the center of the desired circular trajectory. Figure 5.46 shows the desired trajectory and motion the user traced over multiple cycles and Figure 5.47 shows the user error.

The plot of the applied forces necessary under these control conditions is given in Figure 5.48. A large portion of this error is accounted for by a phase shift between the desired trajectory and the user trajectory, as shown in the plots of the $\mathrm{X}$ and $\mathrm{Y}$ tracking over the various cycles in Figure 5.49 and Figure 5.50. Figure 5.51 and Figure 5.52 are the errors associated with each respectively.

The user initially lags the desired location of the reference line, quickly becoming accustomed to the manipulator. The user's error decreases and quickly increases again as the user begins anticipating the required motion. Throughout the entire run, the user tended towards a lower error when tracing the bottom portion of the circle compared to the top, this could simply be due to the location of the circle with respect to the arm of the user, as fine motor control is more difficult the higher the arm is lifted. Alternatively, this could be due to the dynamics model of the manipulator, creating an environment where the end effector is more manoeuvrable in the negative $\mathrm{Y}$ direction, possibly due to incorrect gravity compensation.

The controller was effective in creating an environment where the user could trace the curve. The large damping in the system was applied to create a more stable test platform, as the application of the system is gait rehabilitation where there are significantly higher loads than in this manual manipulation. The resolution and noise of the force torque 
sensor made for an unstable robot with low damping and no dead zone applied to both sensor position data of $5 \mathrm{~N}$.

The sensor data was heavily filtered with a $3 \mathrm{~Hz}$ filter to mitigate the effect of the sensor noise, though this could cause issues with the full application of the robot as a gait platform. This will need to be addressed in future work. From Figure 5.48, it is evident that the loading conditions of this test were not representative of the conditions that will be experienced during gait rehabihtation. Under manual manipulation, the end effector loading reached a maxımum value of approxımately $10 \mathrm{~N}$, or less than 1 percent of the maximum expected end effector loading during a gait control.

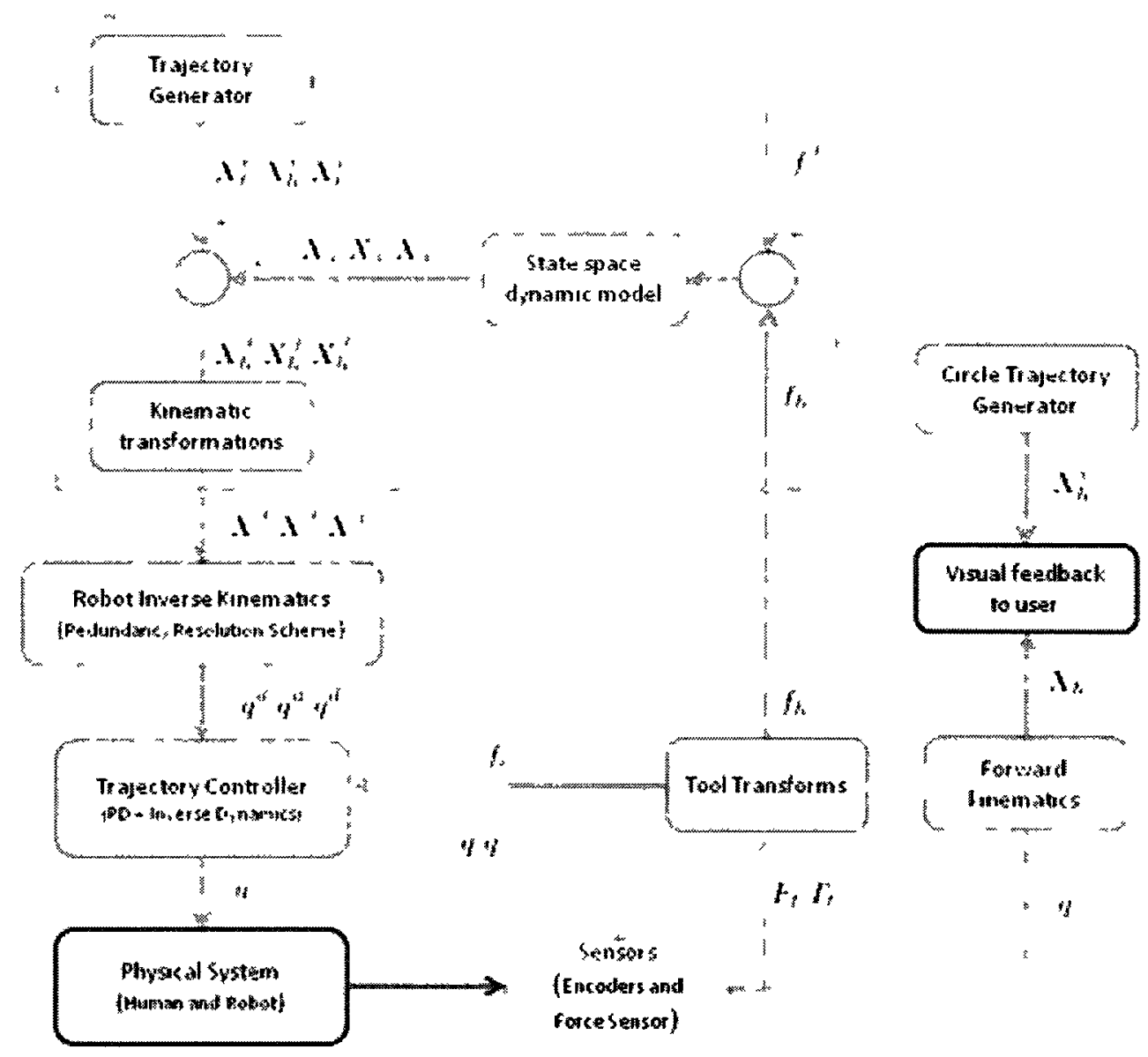

Figure 5.45: Information Flow of Admittance Control Mode 
Table 5.8: Admittance Controller Parameters

\begin{tabular}{lll}
\hline Parameter & Value & Units \\
\hline$M_{I}$ & {$[1,1,0.1]$} & $\left(\mathrm{kg}, \mathrm{kg}, \mathrm{Nm}^{2}\right)$ \\
$B_{I}$ & {$[150,150,9]$} & $(\mathrm{Ns} / \mathrm{m}, \mathrm{Ns} / \mathrm{m}, \mathrm{Ns} / \mathrm{rad})$ \\
$K_{I}$ & {$[0,0,30]$} & $(\mathrm{N} / \mathrm{m}, \mathrm{N} / \mathrm{m}, \mathrm{Nm} / \mathrm{rad})$ \\
$f_{h}^{d}$ & {$[0,0,0]$} & $(\mathrm{N}, \mathrm{N}, \mathrm{Nm})$ \\
$X_{h}^{r}$ & {$[0.7,0.35,0]$} & $(\mathrm{m}, \mathrm{m}, \mathrm{rad})$ \\
$\dot{X}_{h}^{r}$ & {$[0,0,0]$} & $(\mathrm{m} / \mathrm{s}, \mathrm{m} / \mathrm{s}, \mathrm{rad} / \mathrm{s})$ \\
$\ddot{X}_{h}^{r}$ & {$[0,0,0]$} & $\left(\mathrm{m} / \mathrm{s}^{2}, \mathrm{~m} / \mathrm{s}^{2}, \mathrm{rad} / \mathrm{s}^{2}\right)$ \\
$r_{c}$ & 0.1 & $(\mathrm{~m})$ \\
\hline
\end{tabular}

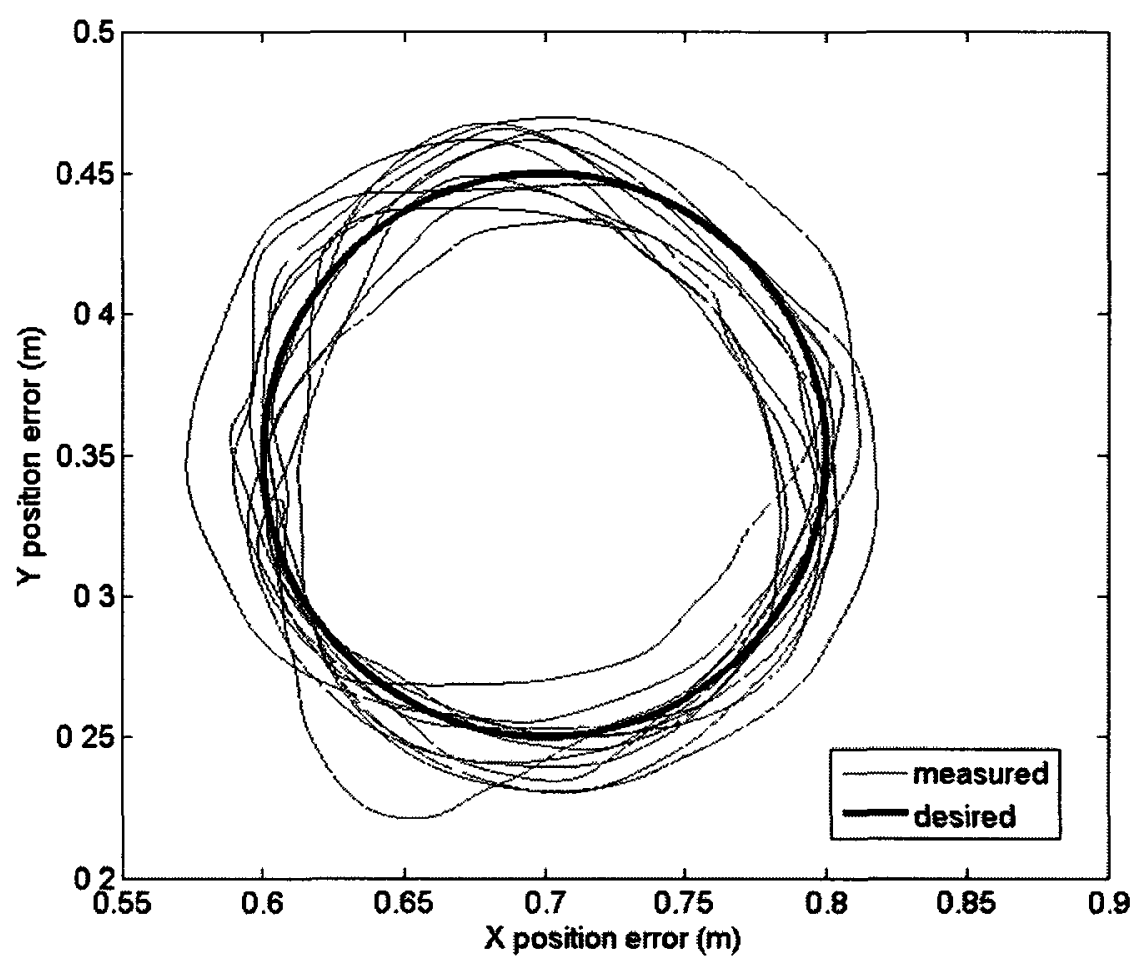

Figure 5.46: End Effector Tracking Results of Admittance Controller 


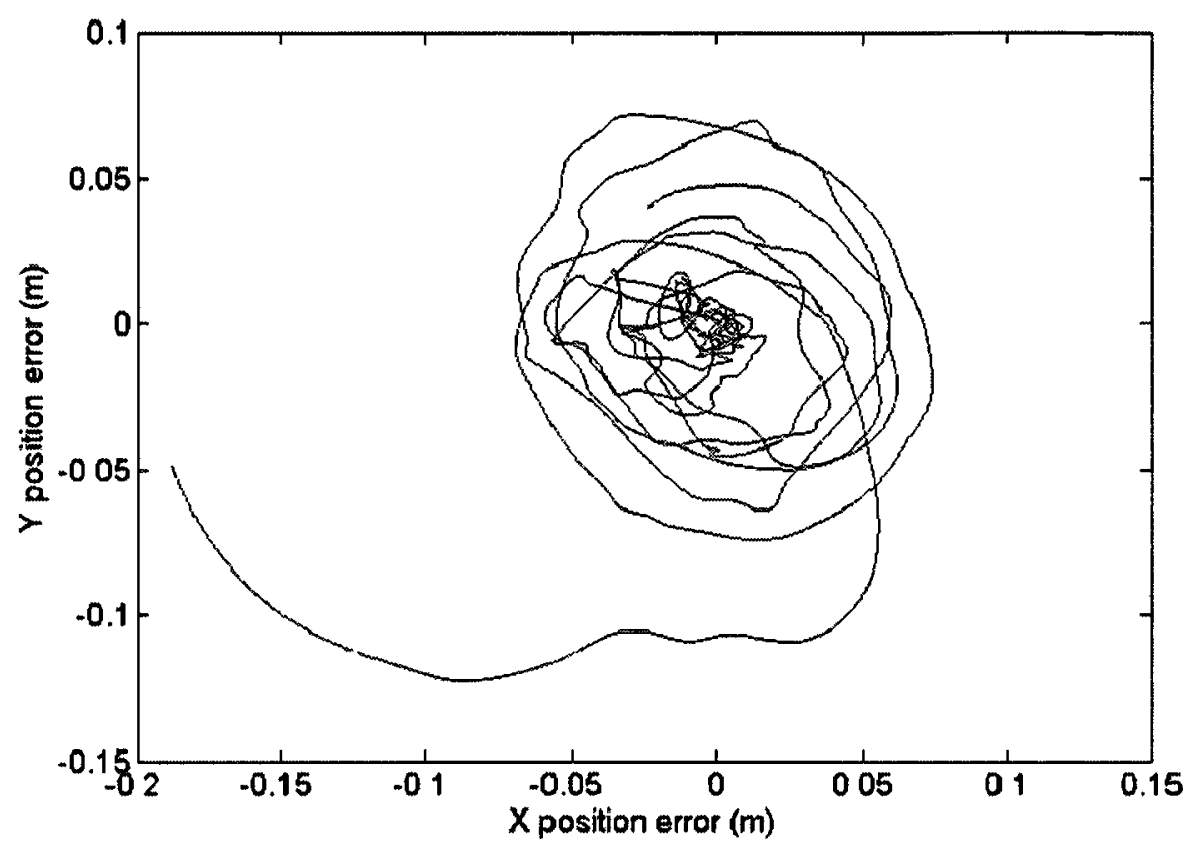

Figure 5.47: End Effector Tracking Error of Admittance Controller

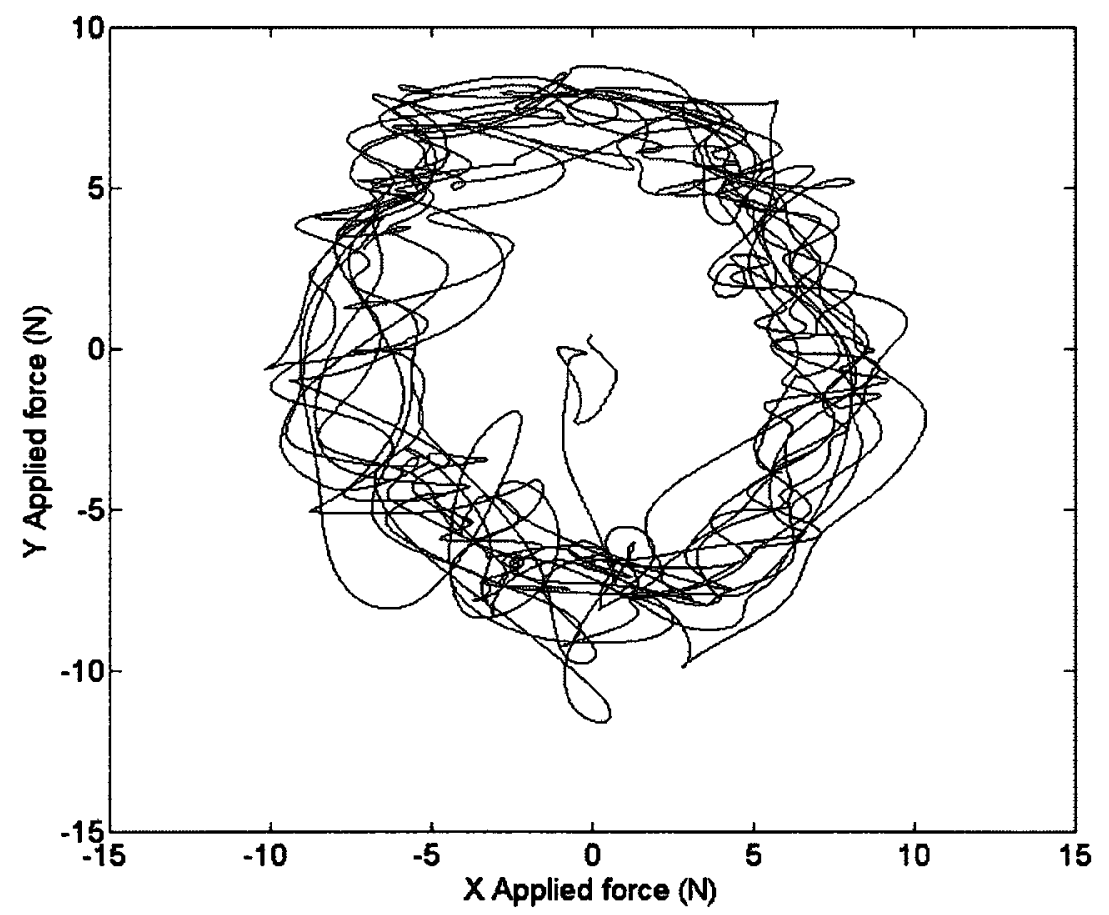

Figure 5.48: Applied Forces during Admittance Control Experiment 


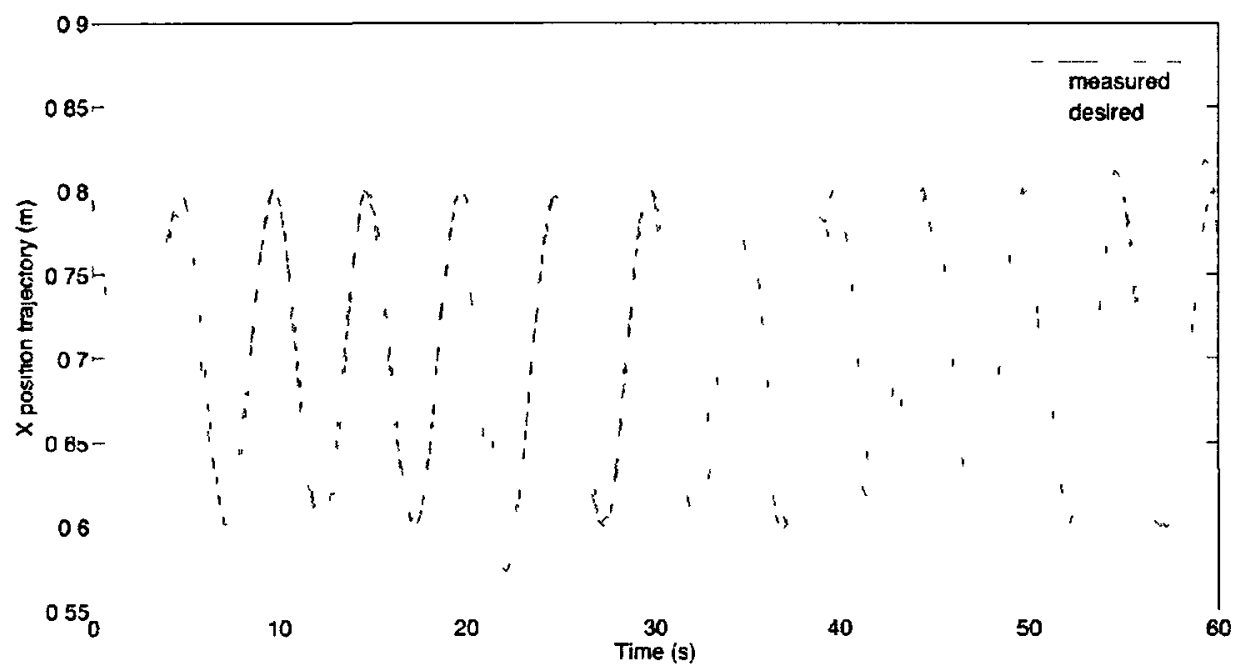

Figure 5.49: X Position Tracking of Admittance Controller

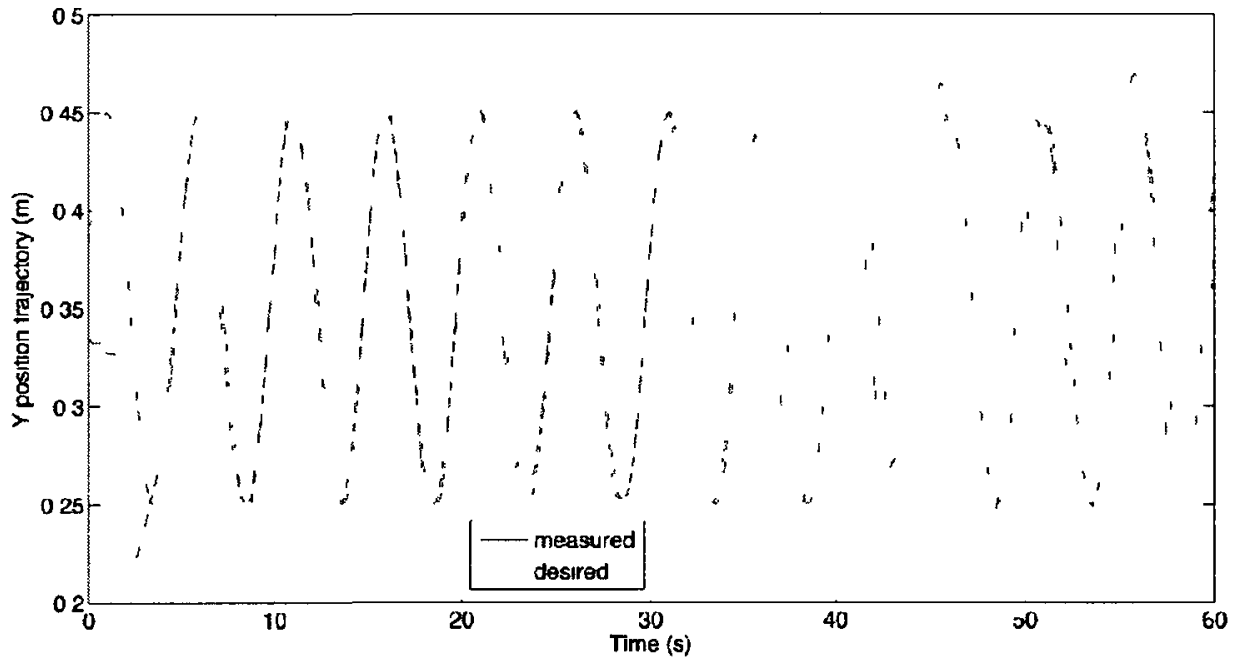

Figure 5.50: Y Position Tracking of Admittance Controller 


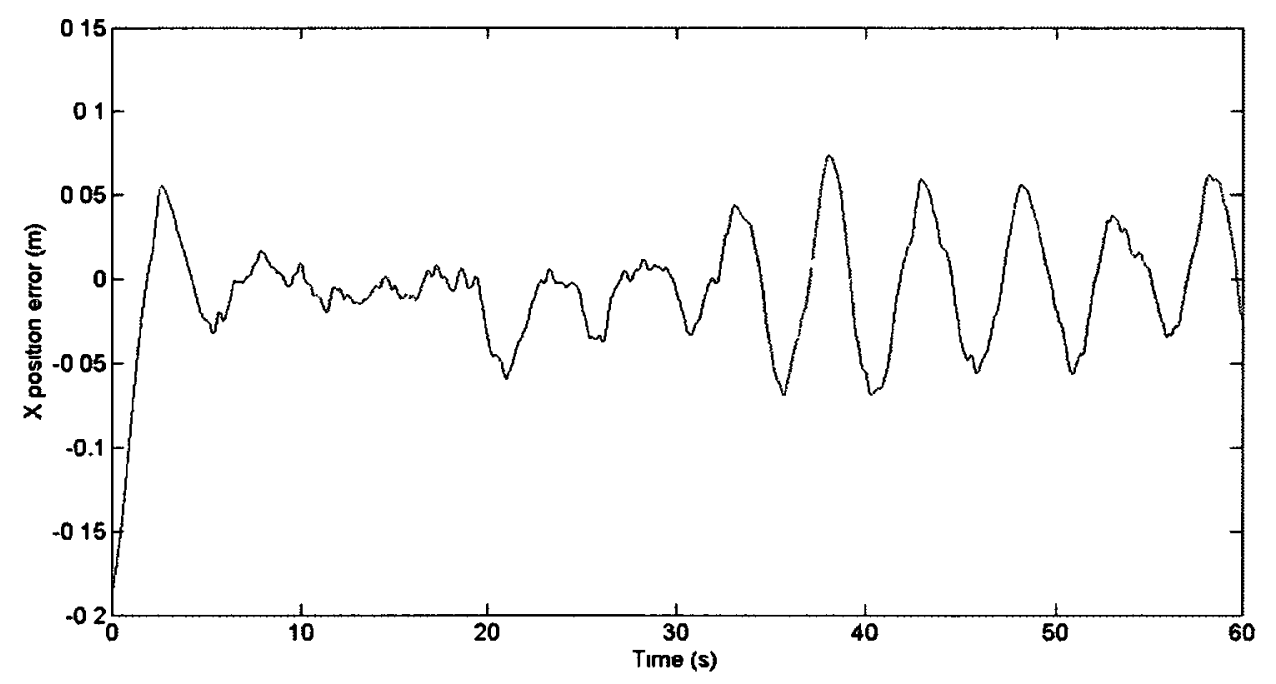

Figure 5.51: X Position Tracking Error of Admittance Controller

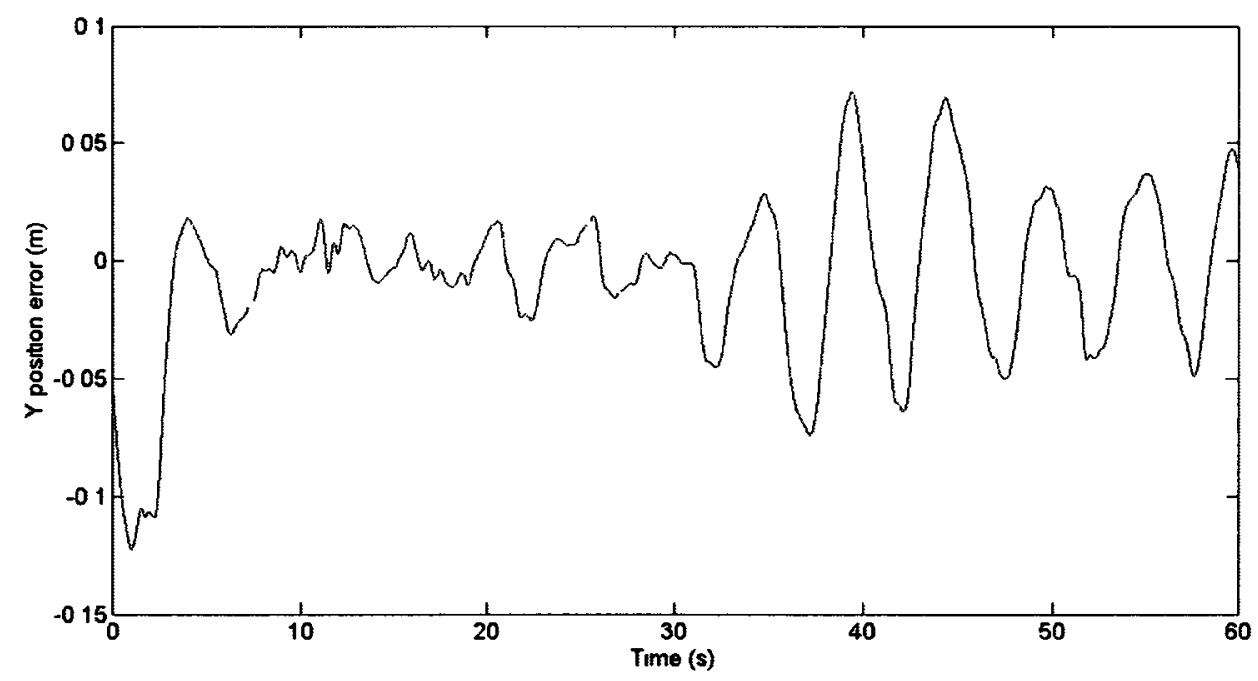

Figure 5.52: Y Position Tracking Error of Admittance Controller

\subsection{Summary}

This chapter highlighted the calibration process, both with single degree of freedom and with the assembled four degree of freedom manipulator and kinematic calibration of the 
robot. The second portion of the chapter presented the testing performed to verify the controller and manipulator after the mechanical and electrical/software components were all integrated. Joint space, task space and force controlled admittance model experiments were performed and analyzed. 


\section{Chapter 6: Conclusions and Future Work}

The ViGRR platform was developed to investigate haptic gait rehabilitation control algorithms. Robotics offer an opportunity for large advancements to be made in the field of rehabilitation as the need for repetitive, specific motions puts high demands on physiotherapists. To reduce the load on a physiotherapist, many current platforms are modelled on the BWSTT design, which supports the patient's weight in an upright position and using the treadmill to create a moving ground. The use of a haptic manipulator allows for the inclusion of rehabilitation methods focussed on additional tasks such as ascending and descending a flight of stairs, or emulating uneven ground. By articulating the foot and not constraining the motion of the leg with an anthropomorphic design, the user is not inhibited in their motion, allowing for a more realistic emulation of standard gait. The goal of this thesis was to develop a manipulator for use in future developments of haptic control methods for gait training in patients with impaired use of their legs.

\subsection{Conclusions}

From the formulation of the design problem and the analysis of other robotic platforms, through the design and finally the testing the ViGRR prototype platform was successfully developed. The remainder of this section provides an overview of the completed work.

\subsubsection{Analysis of Current Technology}

The current robotic gait rehabilitation platforms either on the market or in development were evaluated using Kleim's ten principles of motor learning. From this a niche for the ViGRR platform was forged as a reclined position haptic manipulator. This requires 
reduced attention prior to and after rehabilitation sessions as a body weight support system is not used.

\subsubsection{Requirements}

From the data presented by Winter [19] and accounting for gravity compensation to support the leg the end effector loading spectrum and trajectories for the ViGRR were determined. The requirements are listed in Section 3.4.

\subsubsection{Structural Design}

From the requirements a thorough optimization process was followed to determine the system design parameters, including the link lengths and offset angle of the end effector and coefficients of the redundancy resolution scheme. This optimization was also used to determine the optimum drive components for the platform. The optimized link dimensions are listed in Table 3.4 and the selected components are listed in Table 3.5.

Using the finite element suite Pro/Mechanica, multiple link cross sections and designs were analysed. The joint structure was iterated to select a highly rigid, light weight structure. The final joint design is shown in Figure 4.7 and the dimensions are listed in Table 4.5.

The various safety features of the manipulator were developed to mitigate injury to the user in the event of an instability in the controller. A magnetic attachment as well as a mechanical release device were designed to handle worst case situations and prevent injury to the patient.

\subsubsection{Manipulator and Structural Analysis}

The solid model structure designed in Pro/Engineer Wildfire 4.0 was analyzed using the finite element suite Pro/Mechanica. Under a worst case static load condition, the end effector deflected $17.3 \mathrm{~mm}$ with a maximum von Mises stress of $467 \mathrm{MPa}$. Though this load exceeds the ultimate strength of the Aluminum $6061 \mathrm{~T} 6$, the design point represents a transient point in the gait trajectory of a healthy $95^{\text {th }}$ percentile male loading the manipulator with the loads of a healthy patient. This was taken as the worst possible user 
as patients requiring rehabilitation will not fully load the structure with $100 \%$ of the ground reaction forces. The maximum deflection is within the requirements.

The manipulator workspace contains the workspace of both the $5^{\text {th }}$ percentile female and the $95^{\text {th }}$ percentile male joint space. The results of the kinematic test are in Figure 4.26.

\subsubsection{Calibration}

The kinematic properties and dynamic parameters of the manipulator were determined through three calibration tests. The link lengths were determined through self motion of the redundant manipulator and recording the joint angles in nine different configurations. A least squares fit of the joint data provided the link dimensions from a known end effector location. The dynamic parameter identification was performed in two steps. Initially the single degree of freedom parameters were determined by articulating the disassembled pieces of the manipulator. These parameters were used as initial values for the four degree of freedom identification. The results of the dynamic calibration are shown in Section 5.4. The errors in the 4 degree of freedom calibration are less than 5\% and these points represent points in the trajectory where the manipulator is changing direction and high torques were applied.

\subsubsection{Experimentation}

The completed, calibrated ViGRR robot was run in three control modes to determine the accuracy of the controllers and to confirm the functionality of the actuation components. Future work with high loading conditions will be used to verify the structural design.

In the joint space control, the errors of the joint angles did not exceed 0.5 degrees throughout the motion, Table 5.5. In the task space control, the end effector error during a circular trajectory did not exceed 1.5 millimeters, while the average error was less than half a millimeter. The max orientation error of the end effector was sub-degree, Table 
5.7. In applications to leg manipulation, these results are acceptable as these represent transient errors during motion of the manipulator.

The admittance control mode was used to test the force feedback where the user manipulated the end effector along a desired trajectory. This experiment was performed to evaluate the task specific control of the robot as future design will focus on gait rehabilitation specific task control.

\subsection{Future Work}

The ViGRR platform meets all design requirements as a prototype platform and the experiments verify that the design concept is feasible. The development of the robot into a final product though, requires multiple modifications.

\subsubsection{Robot Development}

With the current calibration and orientation of the Force/Torque sensor there is a $4 \mathrm{~N}$ band of noise in the $\mathrm{X}$ direction of the end effector. As more accurate calibrations are available, a calibration with a lower maximum loads and higher resolution can be applied. The end effector could be redesigned to have the force torque sensor placed in line with the final link. This would load the end effector significantly laterally and in torque. The feasibility of this would need to be determined through a study of the loading conditions.

A study focussing on the design of the linkages with the use of composite materials

could greatly decrease the end effector deflection while also decreasing the mass and inertia of the links.

The use of harmonic drives in the joints adds flexibility to the structure. As this may decrease the sensation from the haptic interface, investigation into the validity of using flexible joints in the haptic manipulator is recommended.

Currently, the manipulator is attached to an office desk with an inch thick board of MDF added to increase the stiffness of the base. A future design consideration that was included in the loading conditions for the requirements of the manipulator is the rotation 
of the platform for the patient to be in an upright or angled position during gait rehabilitation. A design of a project specific support structure would provide a stiff platform and allow for modifiable base.

As further development is performed, muscle activation during use of ViGRR will become an important criterion. An investigation of the addition of either a passive or actuated 'roll' joint for the ankle for activation of stabilizer muscles in the patients leg as well as additional actuation to articulate the pelvis of the patient should be pursued. Once the design has been validated for gait training, the addition of a second manipulator for parallel gait training would present the platform as a full gait rehabilitation platform.

\subsubsection{Controller Development and Clinical Trials}

In addition to these design improvements for the structure of the ViGRR, significant work on the development of the control algorthms and advancement of the platform as virtual simulator must be performed. Research into collision models and actualization of the sensation of the movement of the center of pressure during gait pose interesting areas of study.

Ultimately, the goal of the project is to develop a robotic platform that will aid persons in regaining the ability to walk and increase their quality of life. For this to be realized, development relies on collaboration among engineering and medical personnel. Therefore, although it is primarily an engineering platform, medical researchers, doctors, and physiotherapists are all required to bring the project to fruition. 


\section{Chapter 7: Bibliography}

[1] Spinal-cord Injury. McDonald, John W. 9304, s.1. : The Lancet, 2002, The Lancet, Vol. 359, pp. 417-425.

[2] A Report from the American Heart Association Statistics Committee and Stroke Statistics Subcommittee. Lloyd-Jones, Donald et al. s.l. : Hear Disease and Stroke Statistics, 2009.

[3] Report summary: Tracking heart disease and stroke in Canada 2009. Dai, $\mathbf{S}$ and al., et. 4, s.1. : Public Health Agency of Canada, 2009, Vol. 29.

[4] Mitochondria, oxidative metabolism and cell death in stroke. Sims, Neil R and

Muyderman, Hakan. 1, s.l. : Biochimica et Biophysica Acta, 2010, Vol. 1802. 80-91.

[5] Umphred, D. Neurological Rehabilitation. s.l. : Mosby, 2006.

[6] Brain Plasticity and Stroke Rehabilitation. Johansson, Barbro B. Nashville, Tenn : American Heart Association, 2000.

[7] Plasticity of motor systems after incomplete spinal cord injury. Raineteau, Olivier and Schwab, Martin E. s.l. : Nature Reviews Neuroscience, 2001, Vol. 2.

[8] Motor rehabilitation using virtual reality. Sveistrup, Heidi. Ottawa : Journal of Neuroengineering and Rehabilitation, 2004, Vol. 1.

[9] Multiple sclerosis: a preliminary study of selected variables affecting rehabilitation outcome. Langdon, D W and Thompson, A J. 2, s.1. : Multiple Sclerosis, 1999, Vol. 5. [10] Evaluation and rehabilitation of patients with adult motor neuron disease. Francis, Kathleen, Bach, John R and DeLisa, Joel A. 8, s.l. : Archives of Physical Medicine and Rehabiltiation, 1999, Vol. 80. 
[11] Long-term plasticity at GABAergic and glycinergic synapses: mechanisms and functional significance. Gaiarsa, Jean-Luc, Caillard, Olivier and Ben-Ari, Yehezkel. 11, s.l. : Trends in Neurosciences, 2002, Vol. 25. [12] Motor cortex plasticity during constraint-induced movement therapy in stroke patients. Liepert, J and al., et. 1, s.l. : Neuroscience Letters, 1998, Vol. 250. [13] Principles of Experience-Dependent Neural Plasticity: Implications for Rehabilitation After Brain Damage. Kleim, Jeffery A. and Jones, Theresa A. s.l. : Journal of Speech, Language and Hearing Research, 2008, Vol. 51.

[14] Stein, Joel. Stroke Recovery and Rehabilitation. s.1. : Demos Medical Publishing, 2009. 1933864125.

[15] Functional Reorganization of Primary Somatosensory Cortex in Adult Owl Monkeys Afer Behaviourally Controlled Tactile Stimulation. Jenkins, William M and al., et. 1, s.1. : Journal of Neurophysiology, 1990, Vol. 63.

[16] Behavioral and neurophysiological effects of delayed training following a small ischemic infarct in primary motor cortex of squirrel monkeys. Barbay, Scott and al., et. 1, s.l. : Experimental Brain Research, 2006, Vol. 169.

[17] Treadmill training with partial body weight support after stroke: a review. Hesse, Stefan. 2008, NeuroRehabilitation, Vol. 23, pp. 55-65.

[18] Northwest Regional Spinal Cord Injury System. [Online] [Cited: September 13, 2010.] http://sci.washington.edu/info/newsletters/articles/03sp_improve_walk.asp.

[19] Winter, D. A. The Biomechanics and Motor Control of Human Gait. s.l. : University of Waterloo Press, 1991.

[20] Patient-cooperative strategies for robot-aided treadmill training: first experimental results. Riener, R., et al. 2005, \#IEEE_J_NSRE\#, Vol. 13, pp. 380-394.

[21] Abnormal joint torque patterns exhibited by chronic stroke subjects while walking with a prescribed physiological gait pattern. Neckel, Nathan D, et al. 2008, J Neuroeng Rehabil, Vol. 5, p. 19. 
[22] Prospective, blinded, randomized crossover study of gait rehabilitation in stroke patients using the Lokomat gait orthosis. Mayr, Andreas, et al. 2007, Neurorehabil Neural Repair, Vol. 21, pp. 307-314.

[23] Clinical assessments performed during robotic rehabilitation by the gait training robot Lokomat. Lunenburger, L., et al. 2005. pp. 345-348.

[24] Multicenter randomized clinical trial evaluating the effectiveness of the Lokomat in subacute stroke. Hidler, Joseph, et al. 2009, Neurorehabil Neural Repair, Vol. 23, pp. 513.

[25] Robotic Orthosis Lokomat: A Rehabilitation and Research Tool. Jezernik, Saso and al., et. 2, s.l. : International Neuromodulation Society, 2003, Vol. 6.

[26] Using a Robotic Gait Orthosis as Haptic Display - A Perception-Based Optimization Approach. Wellner, M., et al. 2007. pp. 81-88.

[27] A Study on Sound Feedback in a Virtual Environment for Gait Rehabilitation. Wellner, Mathias, Schaufelberger, Audrey and Riener, Robert. 2007. pp. 53-56. [28] Alterations in muscle activation patterns during robotic-assisted walking. Hidler, Joseph M and Wall, Anji E. 2005, Clin Biomech (Bristol, Avon), Vol. 20, pp. 184-193. [29] Kinematic trajectories while walking within the Lokomat robotic gait-orthosis.

Hidler, Joseph, Wisman, Wessel and Neckel, Nathan. 2008, Clin Biomech (Bristol, Avon), Vol. 23, pp. 1251-1259.

[30] Advances in the understanding and treatment of stroke impairment using robotic devices. Hidler, Joseph, et al. 2005, Top Stroke Rehabil, Vol. 12, pp. 22-35.

[31] Treadmill Training. [Online] [Cited: September 15, 2010.] http://www.healingtherapies.info/treadmill_training.htm. [32] Synchronous stimulation and monitoring of soleus $H$ reflex during robotic body weight-supported ambulation in subjects with spinal cord injury. Querry, Ross G, et al. 1, s.l. : Journal of Rehabilitation Research and Development, 2008, Vol. 45. [33] LOPES: selective control of gait functions during the gait rehabilitation of CVA patients. Ekkelenkamp, R., Veneman, J. and der, H. van. 2005. pp. 361-364. 
[34] Design and evaluation of the LOPES exoskeleton robot for interactive gait rehabilitation. Veneman, Jan F, et al. 2007, IEEE Trans Neural Syst Rehabil Eng, Vol. 15 , pp. 379-386.

[35] The Effects on Kinematics and Muscle Activity of Walking in a Robotic Gait Trainer During Zero-Force Control. F, Edwin H, et al. 2008, IEEE Trans Neural Syst Rehabil Eng, Vol. 16, pp. 360-370.

[36] Improvement of rehabilitation possibilities with the MotionMaker TM. Metrailler, P., et al. 2006. pp. 359-364.

[37] Novel Gait Adaptation and Neuromotor Training Results Using an Active Leg Exoskeleton. Banala, S. K., et al. 2010, \#IEEE_J_MECH\#, Vol. 15, pp. 216-225. [38] The WalkTrainer: A Robotic System for Walking Rehabilitation. Bouri, M., et al. 2006. pp. 1616-1621.

[39] Safety concept for robotic gait trainers. Schmidt, H., Hesse, S. and Bernhardt, R. 2004. Vol. 1, pp. 2703-2706.

[40] Innovative gait robot for the repetitive practice of floor walking and stair climbing up and down in stroke patients. Hesse, Stefan, Waldner, Andreas and Tomelleri, Christopher. 2010, J Neuroeng Rehabil, Vol. 7, p. 30.

[41] Upper and lower extremity robotic devices for rehabilitation and for studying motor control. Hesse, Stefan, et al. 2003, Curr Opin Neurol, Vol. 16, pp. 705-710.

[42] McDowell, M. A., et al. Anthropometric Reference Data for Children and Adults: United States, 2003-2006. U.S. Department of Health and Human Services. 2008. National Health Statistics Report.

[43] Dempster, W. T. Space requirements of the seated operator. United States Air Force, Wright Patterson Air Force Base. 1955.

[44] Identification of passive elastic joint moments in the lower extremities. Riener, $\mathbf{R}$. and Edrich, T. 1999, J Biomech, Vol. 32, pp. 539-544.

[45] Design and Controls for a Gait Rehabilitation (Thesis). Chisholm, Kyle. s.1. : Carleton University, 2010. 
[46] Spong, Mark W., Hutchinson, Seth and Vidyasagar, M. Robot Modeling and Control. s.l. : Wiley $\backslash \&$ Sons Inc., 2006.

[47] Siciliano, Bruno, et al. Robotics Modelling, Planning and Control. s.l. : SpringerVerlag London Limited, 2009.

[48] Cup Type Component Sets I\& Housed Units: CSF I\& CSG Series. 2006.

[49] AKM Series Motors Selection Guide. 2006.

[50] S200 High Performance Compact Brushless Servo Drives Reference Manual. 2008.

[51] Data Acquisition (DAQ) Six-Axis Force/Torque Sensor System Installation and Operation Manual. 2008.

[52] Six-Axis Force/Torque Transducer System Calibration Accuracy Report. 2009.

[53] Q8 Data Acquisition System User's Guide. 2003.

[54] Q8 High-Performance H.I.L. Control Board Product Information Sheet. 2008. 